\title{
Hypoxia in experimental atherosclerosis
}

\author{
Citation for published version (APA):
}

Marsch, E. (2016). Hypoxia in experimental atherosclerosis: linking cellular oxygen sensors and cholesterol metabolism . [Doctoral Thesis, Maastricht University]. Maastricht University. https://doi.org/10.26481/dis.20160518em

Document status and date:

Published: 01/01/2016

DOI:

10.26481/dis.20160518em

Document Version:

Publisher's PDF, also known as Version of record

\section{Please check the document version of this publication:}

- A submitted manuscript is the version of the article upon submission and before peer-review. There can be important differences between the submitted version and the official published version of record.

People interested in the research are advised to contact the author for the final version of the publication, or visit the DOI to the publisher's website.

- The final author version and the galley proof are versions of the publication after peer review.

- The final published version features the final layout of the paper including the volume, issue and page numbers.

Link to publication

\footnotetext{
General rights rights.

- You may freely distribute the URL identifying the publication in the public portal. please follow below link for the End User Agreement:

www.umlib.nl/taverne-license

Take down policy

If you believe that this document breaches copyright please contact us at:

repository@maastrichtuniversity.nl

providing details and we will investigate your claim.
}

Copyright and moral rights for the publications made accessible in the public portal are retained by the authors and/or other copyright owners and it is a condition of accessing publications that users recognise and abide by the legal requirements associated with these

- Users may download and print one copy of any publication from the public portal for the purpose of private study or research.

- You may not further distribute the material or use it for any profit-making activity or commercial gain

If the publication is distributed under the terms of Article $25 \mathrm{fa}$ of the Dutch Copyright Act, indicated by the "Taverne" license above, 


$$
\begin{gathered}
\text { HyPDXIA IN } \\
\text { EXPERIMENTAL } \\
\text { Atherosclerogss }
\end{gathered}
$$

LINking cellular aXygen sengors

and Cholegterdl MetaboligM 
(C) Elke Marsch, Maastricht 2016.

All rights reserved. No part of this book may be reproduced, stored in a retrieval system of any nature, or transmitted in any form or by any means (electronic, mechanical, photocopying, recording or otherwise) without prior written permission of the author, or when appropriate, by the publishers of the publications.

ISBN/EAN

Cover art \& design:

Layout \& figure design:
9789462953642

Floor Tellers, Rens Tellers, Oscar Janssen

Tammy Oth, Elke Marsch 


\section{HYPOXIA IN \\ EXPERIMENTAL \\ ATHEROSLLEROSIS \\ LINKING CELLULAR DXYGEN SENSORS \\ AND Cholegterol MetablligM}

\section{Dissertation}

to obtain the degree of Doctor at Maastricht University, on the authority of the Rector Magnificus, Prof. dr. L.L.G. Soete in accordance with the decision of the Board of Deans, to be defended in public

on Wednesday, May $18^{\text {th }} 2015$ at 10 o'clock

by

Elke Marsch 
SUPERVISORS

Prof. dr. M.J.A.P. Daemen

Prof. dr. E.A.L. Biessen

CO-SUPERVISOR

Dr. J.C. Sluimer

\section{MEMBERS OF the ASSESSMENT COMMItTEE}

Prof. dr. M.J. Post (chairman)

Prof. dr. L.J. de Windt

Dr. G. H. Goossens

Prof. dr. J.A. Kuivenhoven (University Medical Centre Groningen, The Netherlands)

Prof. dr. A. Tall (Columbia Medical Centre, New York, USA) 


\section{TABLE OF CONTENTS}

$\begin{array}{lll}\text { Chapter } 1 & \text { General introduction } & 7\end{array}$

Chapter 2 Hypoxia in atherosclerosis and inflammation 39

Chapter $3 \quad$ Reversal of hypoxia in murine atherosclerosis prevents $\quad 57$ necrotic core expansion by enhancing efferocytosis

Chapter 4 The effect of prolonged dietary nitrate supplementation on atherosclerosis development

Chapter 5 Deficiency of the oxygen sensor prolyl hydroxylase stimulates non-biliary cholesterol excretion attenuating hypercholesterolemia and atherosclerosis

Chapter 6 Hematopoietic hif-prolyl hydroxylase 3 deficiency aggravates Atherosclerotic plaque development initial findings

Chapter $7 \quad$ General discussion

Addendum Summary / Samenvatting / Zusammenfassung

List of abbreviations

Acknowlegdments / Dankwoord / Danksagung 
„.... könnte der mensch sell leben Nicht Weinen, Ach, WIe ertrüg' er eg da!"

(KLOPSTOCK 1724-180习) 

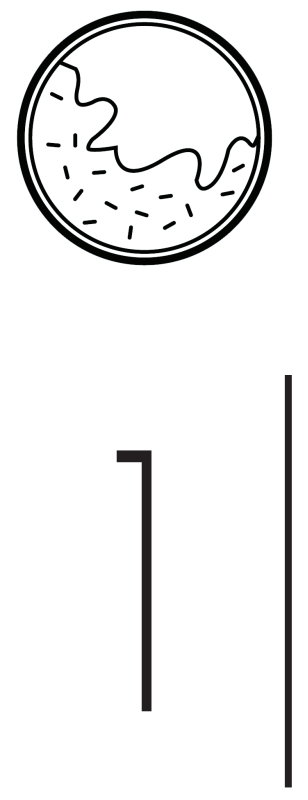

general Introduction 



\section{ATHEROSCLEROSIS - FROM A "CHOLESTEROL" TO A "CHRONIC INFLAMMATORY DISEASE"}

Atherosclerosis, or hardening of the arteries, is a chronic condition in which plaque builds up at the inner lining of an artery and describes sedimentation of cholesterol, fatty substances, cells and cellular debris and waste products, calcium and fibrin, affecting the large and medium-sized arteries (1).

Atherosclerosis is the major underlying cause of coronary artery disease (CAD), peripheral artery disease (PAD) and stroke, which are respectively caused by blood clots in heart, arteries and brain resulting in blood flow obstruction in heart, peripheral tissues and brain. CAD, PAD and stroke cumulatively account for $80 \%$ of all cardiovascular disease (CVD) deaths worldwide. In turn, an estimated 31\% of all deaths worldwide are attributed to CVD (2). Thus approximately one in four deaths worldwide is linked to atherosclerosis (80\% atherosclerosis of 31\% CVD worldwide).

Originally, atherosclerosis was thought of as a cholesterol-driven disease. In as early as 1913, Nikolai Anichkov could show that cholesterol alone induced atheromatous changes in vascular walls of rabbits (3). A finding that was later extended to chickens (4-6). It was Virchow (end 19th century) and later Aschoff (1920s), who revolutionized the field by studying pathophysiology of disease, thus combining careful clinical observation in humans with intelligent experimentation (7). For atherosclerosis this yielded the theory by Aschoff of mechanical damage to the inner lining of the vessel to underlie the entry of blood plasma into the arterial wall (8). Indeed, this notion could be experimentally confirmed by others, linking hypertension with atherosclerosis advancement in hypercholesterolemic dogs (9). It was then indeed realized that only in highly sensitive animals, such as rabbits elevated serum cholesterol levels were sufficient to induce atherosclerosis, whereas in others, e.g. mice, additional triggers were required, including mechanical endothelium injury (e.g. via hypertension), bile salts, vitamin D administration or immunization with foreign proteins (10). Around that time, attention was drawn to the observation that atherosclerosis already develops early on in life, as analyzed in a cohort of young soldiers killed in the Korean War (average age 22), suggesting a chronic, slow disease progression (11).

Based on the cellular composition of atherosclerotic plaques, inflammation was proposed as an additional factor in atherosclerosis in the 1970s (8). Studies in rabbits using antiinflammatory agents confirmed this theory, as they could reduce plaque growth (10). These initial approaches were followed by extensive studies on monocyte/macrophage function in murine models and in vitro studies, clearly showing an inflammatory 
contribution to the disease progression $(8,12,13)$. Taken all the evidence together, atherosclerosis is nowadays considered a chronic lipid-driven, inflammatory disease (14).

Ca. 100 years after the initial studies on atherosclerosis, low-shear stress-mediated subendothelial cholesterol retention and inflammatory changes in the endothelium are believed to initiate the atherogenic process $(15,16)$. In response to low shear stress, injury to the endothelium (e.g. by hypertension), sub-endothelial lipid accumulation or inflammatory triggers, the arterial endothelial layer becomes more permeable, secretes reactive oxygen species (ROS) and cells express adhesion molecules (e.g. VCAM1 , selectins) that capture leukocytes. This, together with changes in composition of the extracellular matrix, can then promote entry and retention of cholesterol-laden lipoproteins (17). Subendothelial lipoproteins are subsequently biochemically modified within the arterial wall and thereafter endocytosed by macrophages, resulting in intracellular cholesterol accumulation (foam-cell formation) and inflammatory activation of cells. In turn, this triggers chemoattractant-mediated leucocyte recruitment to the site of cholesterol retention, namely the intima (luminal layer of the artery). Numerically, monocytes present the most prominent infiltrating cell type. Once inside the lesion, they differentiate to macrophages but also local macrophages can proliferate, adding to the vicious circle of monocyte recruitment - foam cell formation - release of inflammatory cytokines, angiogenic growth factors and ROS - formation of leaky microvessels recruitment of more inflammatory cells. Eventually, (lipid-laden) macrophages and other cells will undergo apoptosis, due to cholesterol overload/ROS production and other stress signals. Under physiological conditions dead cells are cleared by macrophages in a process called efferocytosis. In the event of inefficient efferocytosis, dead cells will undergo "secondary necrosis" and form the lipid-rich necrotic core of atherosclerotic plaques $(18,19)$. Additionally, smooth muscle cells (SMCs) locally proliferate and migrate from the media (middle layer of the artery) into the intima, where they proliferate and produce extracellular matrix molecules, such as collagen and elastin, contributing to the fibrous cap that covers the plaque (20). Up to this stage of atherosclerotic plaque development, atherosclerosis is not associated with clinical symptoms, in parts due to outward remodeling of the vessel wall (21), but also due to the stable nature of the plaque (e.g. large fibrous cap). Rather, most complications arise from rupture of an unstable plaque. Unstable plaques are typically characterized by a thin fibrous cap with a large lipid/necrotic core (22-24). Upon rupture and thus contact of intra-plaque content with the blood stream, an immediate thrombotic response is initiated by platelets (25), resulting in thrombus formation, which might occlude vessels further along the blood stream. 


\section{CHOLESTEROL METABOLISM}

Considering that atherosclerosis is a cholesterol-driven disease, available therapies to reduce atherosclerosis development and cardiovascular risk focus on cholesterol-lowering. Hypercholesterolemia remains a great clinical burden in the western world.

Cholesterol exists in two forms: unesterified and as ester. Unesterified cholesterol contains a charged hydroxyl group, making it amphiphilic with the hydrophilic part being soluble in water/blood. Cholesteryl ester, derived upon esterification of that hydroxyl group, results in a highly lipophilic, thus hydrophobic cholesterol form, which requires to be packaged into the hydrophobic core of lipoproteins to be carried in blood. Principle plasma lipoproteins are chylomicrons, very low density lipoproteins (VLDL), LDL and high density lipoproteins (HDL.) In the context of atherosclerosis, cholesterol contained in chylomicron remnants, VLDL and LDL are considered to forward atherosclerosis, while HDL lipoproteins oppose atherosclerosis development (reviewed in $(26,27)$.

The circulation of cholesterol and triglycerides starts with secretion of triglyceriderich chylomicrons by the enterocyte into the lymph system, from where the particles subsequently enter the blood circulation. Within the enterocyte, microsomal triglyceride transfer protein (MTP) facilitates triglyceride binding to ApoB48 to form chylomicrons (28). While circulating in blood, triglycerides from chylomicrons are hydrolyzed by lipoprotein lipase (LPL), and the chylomicron remnant is subsequently cleared via the LDL receptor-like proteins (LRPs) in the liver (29). In order to further transport triglycerides and cholesterol to tissues, triglycerides are packaged with ApoB100, again under control of MTP (28), and unesterified cholesterol is added to the particle to form the VLDL particle. In the circulation, triglycerides are hydrolyzed again from the VLDL particle via LPL. Also VLDL can then acquire cholesteryl esters from LDL and HDL, with the help of the cholesteryl ester transfer protein (CETP). These processes result in the transformation of VLDL to the smaller cholesteryl ester-rich LDL particle. The small-sized (20-25nm) LDL particle can then cross vascular endothelium, where it supplies tissues with cholesterol or in case of atherosclerosis is oxidized. Additionally, LDL is removed from the circulation via hepatic LDL receptors.

While LDL thus delivers cholesterol to tissue, HDL in turn carries excessive cholesterol from tissue back to the liver. In that sense, HDL forms part in the atheroprotective movement from peripheral tissue to the liver, subsequent excretion via bile and loss though feces, called reverse cholesterol transport (RCT) (30-32). In peripheral tissue, ATP-binding cassette, subfamily A, member 1 (ABCA1) is the rate-limiting step in nascent HDL production, mediating efflux of cholesterol and phospholipid from 
cells to lipid-free ApoA1 (33-37). More recently, also ABCG1 was shown to mediate cholesterol efflux to HDL (38).

\section{TRANS-INTESTINAL CHOLESTEROL EXCRETION}

Next to traditional cholesterol regulation, it has recently been realized that under certain conditions, fecal neutral sterol output can remain persistent, despite surgical or genetic inhibition of biliary cholesterol efflux (see figure 1). This has led to the concept of an additional route of cholesterol excretion, namely non-biliary cholesterol excretion directly from the blood into the intestinal lumen, termed trans-intestinal cholesterol efflux (TICE).

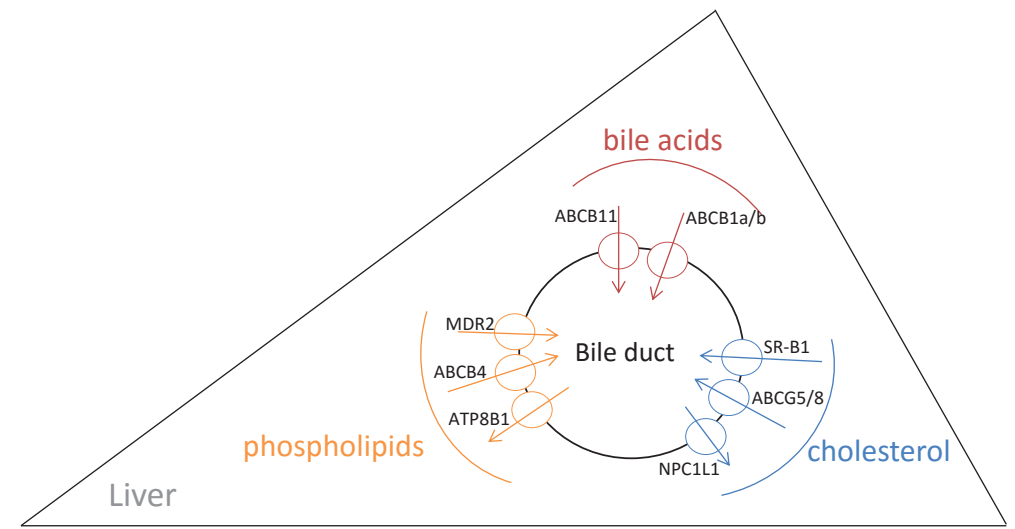

Figure 1: Bile transporters involved in intrahepatic lipid regulation.

Specific transporters are responsible for cholesterol, bile acid or phospholipid excretion into and reabsorption from bile. Deletion of for example ATP-binding cassette subfamily $G$ member 5/8 (ABCG5/8) deficiency results in reduced biliary cholesterol excretion, nonalcoholic fatty liver disease (39) and subsequent upregulation of non-biliary TICE (40). Additionally, enhanced cholesterol-reabsorption from the bile in Niemann-Pick C1-like 1 (NPC1L1) transgenic hepatic overexpression models can enbance TICE (41).

Since the discovery of TICE, its regulatory mechanisms still remain largely unknown. Until now, only high-fat diet (42), plant sterols (43), liver x receptor (LXR) activation (44) and peroxisome proliferator-activated receptor $\delta$ (PPAR $\delta$ ) ligands (45) have been conclusively identified to trigger TICE. A logical site of regulation would be intestinal cholesterol transporters. Indeed, intestinal ABCG5 deficiency could reduce TICE by $40 \%$ (44), while ABCG8 had no effect(46). Additionally, whole body ABCB1a/b deficient mice presented with a $27 \%$ reduction in TICE (47). However, none of these two transporters represent a clear on/off switch for TICE. 
Additional efforts have aimed at identifying the cholesterol-donating lipoprotein for TICE. After initial conflicting data (47), HDL could be excluded as donor for TICE in cholesterol tracing studies (45). In line with this, enhanced ability of VLDL to deliver cholesterol in acyl-CoA: cholesterol O-acyltransferase 2 (ACAT2) deficient mice showed increased fecal neutral sterol excretion without affecting biliary sterol secretion (48), thus suggesting VLDL as cholesterol donor for TICE. Also, LDL remains a likely candidate though, as uptake rates of LDL cholesterol into the intestine are relatively high (49). Receptors for VLDL and LDL include LDLr, VLDLr, LRP and low-density lipoprotein receptor-related protein 8 (LRP8) (also apolipoprotein E receptor 2, apoER2), all of which are expressed in the gut $(50,51)$. However LXR activation, shown to promote TICE and to regulated a great range of target genes in cholesterol, fatty acid and glucose metabolism, can also indirectly target LDLr, VLDLr and apoER2 for proteasomal degradation (52,53). Thus LDLr, VLDLr and apoER2 are again unlikely to be involved in TICE and the receptor for cholesterol uptake from apoB-containing lipoproteins in the intestines for TICE remains unknown.

\section{CURRENT therapy OF ATHEROSCLEROSIS}

Currently available strategies against atherosclerosis progression include surgical removal (carotid endarterectomy), coronary artery bypass grafting and stent-placement, and reduction of risk factors for atherosclerosis. Next to lifestyle changes, this includes adequate hypercholesterolemia, hypertension and diabetes management, with mainly cholesterol lowering therapies being investigated and applied as prevention strategies at this moment:

Epidemiological evidence has linked high high-density lipoprotein-cholesterol (HDL-C) levels with reduced CVD risk and HDL-C raising strategies have therefore been explored already since the 1970s (54). However, the progression of HDL-C raising drugs has been complicated by the great heterogeneity of HDL subpopulations and different features associated with normolipidemic as compared to dyslipidemic state of HDL (55). Probably more importantly, it has become clear that rather than linking absolute HDL-C levels to cardiovascular risk, cholesterol efflux capacity (thus RCT) is the better measure of and target to reduce atherosclerotic burden and cardiovascular incidence (56,57). Actually, multiple HDL-C raising agents, purely enhancing HDL numbers have recently failed in clinical trials: Two CETP inhibitors, raising HDL by inhibiting cholesteryl ester transfer from HDL to VLDL (58), have failed to show effects on coronary events $(59,60)$. A third trial on the CETP inhibitor evacetrapid in atherosclerotic disease was recently discontinued (61). Also trials on niacin, a general 
lipid metabolism regulating but also HDL raising drug, have failed or been aborted due to lack of effects (on top of standard statin treatment) $(62,63)$. Also HDL-mimetic infusion could not affect incidence of major cardiovascular events, such as myocardial infarction, stroke, unstable angina and heart failure (64). One other study showed improved lipoprotein profiles, stimulated cholesterol mobilization and reduced arterial wall dimension and inflammation in familial hypoalphalipoproteinemia patients (65), but long term outcomes remain to be assessed in this study.

Next to HDL-C raising, several low density lipoprotein-cholesterol (LDL-C) lowering therapies are explored at the moment. Indeed, the main drugs used for cholesterollowering in clinical use nowadays are statins, which act by lowering LDL-C. Statins inhibit 3-hydroxy-3-methylglutaryl-coenzyme A (HMG-CoA) expression, the ratelimiting step in cholesterol biosynthesis. The resulting SREBP-dependent feedback response leads to increased LDLr expression by hepatocytes, which in turn can enhance clearance of LDL from the circulation. However, while on the one hand statins are very effective in lowering cholesterol and cardiovascular risk, only fewer than 1 in 3 adults in the US with elevated low density lipoprotein-cholesterol (LDL-C) levels achieve desired LDL-C lowering with statins (66). This is partially due to wrong dosing of statins, but also statin resistance $(67,68)$ and even intolerance has been described (69). Alternatives include proprotein convertase subtilisin-kexin type 9 (PCSK-9) targeting with inhibitors, monoclonal antibodies and antisense methods. PCSK-9 interference inhibits PCSK-9-mediated degradation of LDLr, thereby increasing the concentration of LDLr exposed on the cell surface both in the liver and the periphery. Clinical trials on cardiovascular outcome of PCSK-9 inhibition are ongoing (70), with the first positive outcomes on cholesterol-lowering recently published (71).

As an alternative for lipoprotein modulation, pharmacological targeting of the nonbiliary TICE pathway has recently even been shown to reduce atherosclerosis. Using pharmacological activation of intestine-specific liver $\mathrm{x}$ receptor (LXR), Yasuda et al. could recently enhance "intestinal RCT", thus enhanced RCT via TICE in mice (72). In accordance, an independent study shows increased RCT (biliary and non-biliary) upon intestinal LXR activation with subsequent protection against atherosclerosis in mice (73). Though this is not direct evidence, it is highly suggestive of an anti-atherosclerotic effect of TICE stimulation via LXR activation. Similarly, ezetimibe, an inhibitor of intestinal NPC1L1-mediated cholesterol absorption (74), lowers plasma cholesterol levels without affecting biliary cholesterol excretion, yet increasing fecal neutral sterol output (75). However, in a big clinical trial (IMPROVE-IT), ezetimibe showed only marginal additional reduction in cardiovascular death, major coronary event, or 
non-fatal stroke on top of simvastatin treatment (76). Overall, non-biliary cholesterol excretion and reducing intestinal cholesterol absorption might present valid new targets in reducing plasma cholesterol levels and protecting from atherosclerosis progression. In fact, enhancing biliary cholesterol excretion as in traditional HDL-mediated RCT, might even not be desirable as it promotes cholesterol gallstone disease $(77,78)$.

Next to cholesterol-lowering, recent efforts aim at reducing inflammatory aspects of atherosclerosis, by inhibiting inflammatory mediator biosynthesis and/or signaling or by inducing immune tolerance by means of vaccination. Multiple agents are undergoing clinical trials at the moment (reviewed in (79)), however also here, results are partially disappointing. While most randomized controlled trials on anti-inflammatory agents show reduction in biomarkers, imaging and functional parameters, this does not always translate to improved cardiovascular outcome. In fact, increased rather than reduced cardiovascular risk was observed for cyclooxygenase 2 (COX-2) inhibitors (80), as well as for the nonselective secretory phospholipase A2 (sPLA2) inhibitor, varespladib, aimed to reduce formation of bioactive pro-atherogenic phospholipid generation (81) and for monoclonal antibodies directed against the pro-inflammatory IL-12/IL-23 subunit p40 (82).

Overall, these trials emphasize the complexity of cholesterol metabolism and atherosclerosis development and the need of refinement of current strategies. For example, more detailed knowledge on individual plaque progression and stage (e.g. stable or unstable plaque) might allow more "personalized treatment", thereby rendering current therapies more successful. On top of that, novel strategies targeting cholesterol, inflammation or other pathways in plaque progression are desirable, which naturally need to compete with statins and should thus work independently and as adjuvant for the current gold standard statin intervention.

\section{HYPOXIA AND OXYGEN SENSORS IN ATHEROSCLEROSIS}

A novel strategy to reduce atherosclerotic plaque growth might be targeting the oxygen shortage (hypoxia) within the plaque. Already inn 1974, this concept was introduced, when Vesselinovitch et al. studied plaque regression in rabbits. When switching high cholesterol diet fed rabbits back to normal diet, they could show profound plaque regression. Plaque regression was even more pronounced, when adding oxygen therapy on of top of the diet-switch (83).

The current state-of-literature on hypoxia in atherosclerosis is discussed in more 
detail in chapter 2. In short, already in the 1970s, Heughan et al. could show reduced oxygen tension in atherosclerotic aortas in comparison to healthy controls, by applying microelectrodes to infrarenal aortas in anesthetized rabbits. Intriguingly, lowest oxygen tension values were recorded actually close to the lumen of the plaques (84). This suggests that oxygen diffusion is not the limiting factor for oxygen availability in atherosclerotic plaques in rabbits. Indeed, atherosclerotic plaque hypoxia can result from decreased oxygen diffusion to the core of the plaque, due to fibrous cap formation and plaque expansion, or result from enhanced oxygen consumption by cells within the plaque. Also in mouse models, oxygen diffusion distance is less likely to underlie hypoxia. In fact, the oxygen diffusion distance in LS714T tumors in SCID mice from blood vessel into the interstitial space is about $100-250 \mu \mathrm{m}$. This is taking into account oxygen consumption in the interstitial space (85). Thus, the net oxygen diffusion distance is potentially even bigger. Cellular oxygen consumption is therefore likely to contribute to atherosclerotic plaque hypoxia. More recently, imidazole-derivates, such as pimonidazole were used to detect tissue hypoxia. Imidazoles form adducts with thiol groups in proteins, peptides and amino acids in case of hypoxia $\left(\mathrm{pO}_{2}<10 \mathrm{mmHg}\right)(86)$. Thus at an oxygen saturation below approx. 1\% oxygen (87) imidazole binding can be expected and thiol adducts can ex vivo be analyzed by immunohistochemistry. While detection of a pimonidazole gradient within plaques is difficult, co-localization of imidazoles with inflammatory markers (88), might again suggest enhanced cellular oxygen demand to contribute to plaque hypoxia.

\section{To conclude, plaque hypoxia is present across species (89-91); but a causal role of plaque hypoxia in atherosclerotic plaque development remains to be shown.}

Hypoxia responses can be modulated in two ways: either by enhancing oxygen delivery to the plaque or decreasing oxygen consumption within the plaque.

Enhanced oxygen delivery is already exploited in the clinics for conditions where oxygen flow is obstructed, such as pneumonia, asthma, chronic obstructive pulmonary disease and cystic fibrosis (92). Nowadays, oxygen can be delivered to patients as compressed gas, as liquid (portable oxygen containers) or it can be concentrated from air (oxygen concentration filters). Application of the gas then involved face masks, nasal cannulas, transtracheal oxygen therapy or delivery through breathing-supporting machines, e.g. continuous positive airway pressure (cPAP) devices or ventilators. However, these methods are not very feasible in experimental settings. In turn, hyperbaric oxygen therapy and oxygen gas breathing can be used. In fact, hyperbaric oxygen (HBO) therapy, using $100 \%$ oxygen, has already been shown to reduce atherosclerotic formation in rabbits and 
mice (93-95), though the authors did not study, if the oxygen actually reached plaque tissue. In the clinic, HBO is mainly applied to ischemic conditions, where it could e.g. improve cerebral perfusion (96) and peripheral extremity ischemia (97). Nevertheless, hyperbaric oxygen therapy can result in severe side effects, such as damage to the lungs, oxygen poisoning and build-up of fluid or rupture of the middle ear (98). Therefore, normobaric carbogen gas (95\% oxygen, 5\% carbon dioxide) presents a valid alternative. Carbon dioxide is added to the mixture to prevent pulmonary vasoconstrictive effects of pure oxygen, again a downside of HBO (99). Also in clinics, carbogen gas is applied in hypoxia modification during radiotherapy (100), hearing loss therapy (101) and has recently been tested in brain oxygenation for subsequent cerebral ischemia application (102). In contrast to $\mathrm{HBO}$, carbogen gas has also been shown to improve tissue oxygenation in prostate tumors recently (103). Also, while in radiotherapy, improving oxygenation status allows enough oxygen radicals formation for effective induction of DNA damage in cancer cells, oxygen radical formation or ROS production has not been studied for hearing loss and brain oxygenation. Carbogen gas might thus present a valid method to increase oxygen delivery to plaques in experimental atherosclerosis.

Decreasing oxygen consumption is partly achieved as "natural doping" sportsmen taking beetroot juice already. Beetroot contains high amounts of dietary nitrate, which in turn has been shown to increase mitochondrial efficiency upon conversion to nitrite and nitric oxide in the plasma (104). Thereby, cells use up less oxygen in the electron transport chain, resulting in reduced alterations in tissue oxygenation in skeletal muscle upon exercise and improved exercise performance (104-107). Next to that, however, dietary nitrate has multiple other systemic effects. In fact, one week dietary nitrate and nitrite supplementation reduced infarct size in a murine myocardial ischemia/reperfusion model, not by affecting oxygenation, but by replenishing nitrate and nitrite stores during infarction (108). Also, short term dietary nitrate supplementation has been shown to lower resting blood pressure in humans (109) and dietary nitrate resulted in enhanced cardiorespiratory function (110) and improved glucose tolerance in diabetic rats (111). Further, an anti-inflammatory effect has been linked to dietary nitrate and nitrite consumption (112). All these effects hint towards an atherosclerosis-protective role of dietary nitrate supplementation, which has not yet been studied. However, also discrimination between the different effects and their relative contribution to atherosclerosis is difficult. 


\section{OXYGEN SENSORS IN CARDIOVASCULAR DISEASE}

An alternative to reduce cellular oxygen consumption is cellular reprogramming. Recently, deficiency of the HIF-prolyl hydroxylase 1 (PHD1) deficiency has been described to reduce cellular oxygen consumption in skeletal muscle (113). PHDs in general are prolyl hydroxylases, where they belong to the family of ferrous iron $\left(\mathrm{Fe}^{2+}\right)$ and 2oxo-glutarate (2-OG) dependent dioxygenase enzymes. PHDs play an important role in oxygen sensing, as on top of $\mathrm{Fe}^{2+}$ and 2-OG, the enzymes require oxygen for their enzymatic activity in hydroxylating hypoxia-inducible factor $\alpha(\mathrm{HIF}-\alpha)$ subunits at two (oxygen-dependent degradation domains) proline 402 and 564. This hydroxylation targets HIF- $\alpha$ for von Hippel-Lindau (vHL) protein-mediated ubiquitination and proteasomal degradation (114). In turn, during hypoxic conditions, PHDs lose their activity of degrading HIF, thereby starting a HIF-mediated program on cellular adjustment to hypoxia. Via stabilization of mainly HIF-2 $\alpha$, PHD1 deficiency has been shown to reduce cellular oxidative phosphorylation and increase anaerobic cellular glycolysis (113).

HIFs are a family of basic helix-loop-helix/PAS 9PER/ARNT/SIM) transcription factors, with HIF-1, HIF-2 and HIF-3 described so far. Transcriptionally functional HIF is a heterodimer, composed of HIF- $\alpha$ and HIF- $\beta$ subunits, with all three HIF homologs sharing the same $\beta$-subunit (115). In most cell types, HIF-1 $\alpha$ is stabilized upon acute hypoxia (116-118). HIF-1 $\alpha$ upon heterdimerization mediates transcription of target genes involved in angiogenesis, glucose metabolism, growth and survival, invasion and metastasis and others, thereby adjusting to hypoxic conditions (119). HIF-2 $\alpha$ and HIF-3 $\alpha$ have a more restricted expression pattern in acute hypoxia, partially share target genes, with HIF-1 $\alpha$ being the predominant glycolysis inducer (120-122).

While HIF has been extensively studied in atherosclerosis in a cell-type specific manner, oxygen sensor PHD inhibition has not yet been investigated in this context. The first homolog of PHD was identified in 2001 in c. elegans in 2009 (egg-laying abnormal 9, or egl nine homolog EGLN) (114). Four isoforms have subsequently been discovered: PHD1, 2, 3, 4 and the related enzyme Factor-inhibiting HIF (FIH) (123).

Oxygen levels are the most ubiquitous factor controlling PHD activity. Naturally also availability of co-factors $\mathrm{Fe}^{2+}$ and 2-OG determines catalytic activity of PHDs. For example, ROS can chelate and oxidize PHD bound $\mathrm{Fe}^{2+}(126,127)$. In line, competitive inhibition of 2-OG, by TCA cycle-generated fumarate and succinate, which compete with 2-OG binding to the active site of PHDs, has been described (128). Next to this regulation at enzyme/protein level, PHD2 and PHD3 genes contain hypoxia-responsive elements $(129,130)$, showing transcriptional regulation of PHDs. Also, PHD1 and PHD3 
can be targeted for poly-ubiquitination and proteasomal degradation by Siah1a/2 (131). In fact, Siah2 is actually upregulated in hypoxia, therefore contributing to accelerated degradation of mainly PHD3 (131). In turn, PHD3 is also the isoform with greatest transcriptional upregulation in hypoxia, again presenting a tightly regulated feedback loop system of PHD regulation.

Structure function analyses have revealed great homology of PHDs $1-3$ in the ferrous iron binding catalytic domain. Yet, PHD isoforms have diverse affinity for HIF-1 $\alpha$ hydroxylation (132). Also, while all PHDs hydroxylate HIF prolines at highly-conserved LXXLAP sequence motifs, in vitro systems reveal similar to high activity of PHD2 and PHD3, and lower activity of PHD1 (132,133). Additionally, only PHD1 and PHD2 can hydroxylate proline 402 at a $\mathrm{Km}$ value that exceeds that for proline 465 hydroxylation by 20 to 50 times (133). Also, PHD1 and PHD3 are more active on HIF-2 $\alpha$ than HIF$1 \alpha$, whereas PHD2 hydroxylates HIF-1 $\alpha$ more efficiently (134). Intracellular localization of the enzymes is also distinct. PHD1 is exclusively localized to the nucleus, PHD2 predominantly found in the cytoplasm and PHD3 is detectable uniformly in both cytoplasm and nucleus (135). Differential function might thus be explained by structural differences in the N-termini of the three enzymes and/or unrevealed differences in transcriptional/ (post-) translational regulation.

Next to HIF stabilization, different substrates or at least binding partners of PHDs have been identified. PHD1 an PHD2 have been shown to regulate transcriptional activity of $\mathrm{NK} \varkappa \mathrm{B}$ in a HIF- $\alpha$-independent fashion (136), by hydroxylating IKK $\beta$. PHD1 in turn could be shown to destabilize FOXO3a and consequently, loss of PHD1 led to accumulation of FOXO3a (137). Also activating transcription factor 4 (ATF4) is oxygen-dependently regulated by PHD3, where PHD3 silencing resulted in ATF-4 stabilization under normoxic conditions (138). Next to that, the $\beta 2$-adrenergic receptor could be hydroxylated by PHD3 (139) and also PKM2 hydroxylation by PHD3 has been described to increase binding of PHD3 with HIF, thereby enhancing transactivation of HIF-1 target genes (140). However, direct hydroxylation of HIF-independent factors has been difficult to demonstrate, possibly due to technical factors. 


\section{PHDS IN MYOCARDIAL INFARCTION AND ISCHEMIA/REPERFUSION INJURY}

Due to their oxygen sensing function, PHDs have up to date mainly been studied in ischemia/reperfusion models. Indeed, general PHD inhibition (pan-PHD inhibition) using the iron-chelator Dimethyloxallyl Glycine (DMOG) could reduce myocardial infarct size in mice and rabbits as studied by multiple groups $(141,142)$. Additionally, pre- and post- conditional administration of mice undergoing myocardial infarction with another PHD inhibitor (2-(1-chloro-4-hydroxyisoquinoline-3-carboxamido) acetate, ICA) resulted in HIF- $\alpha$ stabilization in heart muscle cells and reduced infarct size (143). However, the diversity in PHD isoform function suggests differential function in physiology and pathology. Indeed, Rohrbach et al. could show differential expression of PHDs in ischemic hearts of mice and men, with induction of PHD3 in ischemic as well as healthy ageing hearts, suggesting differential function (144). Also under physiological conditions, PHD3 expression is highest in the heart compared to PHD1 and PHD2 (145). Infarcted rat hearts on the other hand mainly express PHD2 and PHD3 (both on mRNA and protein) rather than PHD1 (146), however rat studies should be handled with caution with respect to PHDs, as they express a truncated from of PHD3.

\begin{tabular}{|c|c|c|c|c|c|}
\hline PHD & Manipulation & Model & Outcome & Process & Refs \\
\hline \multirow[t]{5}{*}{ PHD2 } & Cardiac-specific & $\mathrm{Ml}$ & reduced infarct size & increased capillary area & $(153,154)$ \\
\hline & haplodeficiency & stroke & $\begin{array}{l}\text { improved cerebral blood } \\
\text { flow }\end{array}$ & ND & (155) \\
\hline & hypomorphism & hindlimb ischemia & reduced ischemia & enhanced capillary size (not number) & (156) \\
\hline & sh interference & hindlimb ischemia & enhanced neovascularization & angiogenesis induced & (157) \\
\hline & haplodeficiency & hindlimb ischemia & reduced ischemia & $\begin{array}{l}\text { macrophage skewing to } \mathrm{M} 2 \text { and } \\
\text { arteriogenesis }\end{array}$ & (158) \\
\hline \multirow[t]{6}{*}{ PHD1 } & sh interference & hindlimb ischemia & $\begin{array}{l}\text { Less neovascularization } \\
\text { compared to PHD2\&3 }\end{array}$ & - & (157) \\
\hline & wb KO & hindlimb ischemia & reduced ischemia & switch to glycolysis, less ROS & (113) \\
\hline & wb KO & hindlimb ischemia & $\begin{array}{l}\text { recovery of perfusion and } \\
\text { motor function }\end{array}$ & increased capillary density & (159) \\
\hline & wb KO & Ml ex vivo & reduced infarct size & reduced apoptosis and ROS & $(160)$ \\
\hline & wb KO & stroke & reduced infarct size & $\begin{array}{l}\text { switch to oxidative pentose } \\
\text { phosphate pathway scavenging ROS }\end{array}$ & (161) \\
\hline & $\begin{array}{l}\text { wb KO / } \\
\text { hepatic silencing }\end{array}$ & liver ischemia & reduced ischemia & reduced oxidative stress & (162) \\
\hline \multirow[t]{3}{*}{ PHD3 } & wb KO & $\mathrm{Ml}$ & $\begin{array}{l}\text { reduced infarct size/ } \\
\text { preserved function }\end{array}$ & increased capillary density & (163) \\
\hline & sh interference & hindlimb ischemia & enhanced neovascularization & angiogenesis induced & $(157)$ \\
\hline & Cardiac KI & $\mathrm{Ml}$ & enhanced infarct size & Reduced HIF accumulation & (164) \\
\hline
\end{tabular}

Table 1: PHDs in myocardial infarctiona and ischemia/reperfusion injury.

KO - deficiency; KI - knockin; ND - not determined; MI - myocardial infarction; WB - whole body; sh - short bairpin interference. 
The respective effects of PHD isoform inhibition on ischemia/reperfusion injury and the pathways involved are summarized in table 1. Overall, PHD2 and PHD3 deficiency reduce ischemic injury by promoting vessel formation ensuring oxygen supply, whereas PHD1 deficiency protects from ischemia by adjusting cellular metabolism to less ROS production.

As a note of caution on PHD2 inhibition in cardiovascular disease though, there are also reports on cardiac-specific PHD2 inactivation causing cardiomyopathy over time (147) as well as premature lethality of PHD2 whole body deficient mice and development of dilated cardiomyopathy in tamoxifen-induced PHD2 deficiency (injections near term and 3 weeks after birth) (148). Also, PHD2 deficiency is a major inducer of HIF-dependent erythropoiesis (149). While PHD2 inhibition is thus beneficial in anemia patients (see table 2), in subjects with normal erythrocyte counts, raising erythropoiesis and elevated hematocrit could potentially thwart the beneficial cardiovascular effects by possible effects on thrombosis and microvascular perfusion (150-152). Alternatively, as these negative effects are mainly seen in complete PHD2 deficiency models, haplodeficiency and thus partial inhibition might be a valid strategy to circumvent side effects.

\section{translatiONAL POTENTIAL OF PHO INHIBITION}

Current strategies to inhibit PHDs involve mimicking 2-OG, chelating/substituting $\mathrm{Fe}^{2+}$ and blocking the active sites of PHDs. However, making these inhibitors specific for a single PHD isoform remains a challenge. A recent overview of general non-specific PHD inhibitors, principle of inhibition, specificity and therapeutic application is presented in (165). Most general PHD inhibitors in clinical trials nowadays are designed for anemia treatment (table 2). While these data show translational potential and general safety of PHD inhibition, erythropoiesis effects do have to be considered in above mentioned cardiovascular implications of PHD inhibitors. 


\begin{tabular}{|c|c|c|c|}
\hline Inhibitor & Patient Population & Purpose of study & Refs \\
\hline $\begin{array}{l}\text { Vadadustat } \\
\text { (AKB-6548) }\end{array}$ & $\begin{array}{l}\text { Non-dialysis-dependent anemic patients with } \\
\text { chronic kidney disease (Phase III, recruiting) }\end{array}$ & $\begin{array}{l}\text { Reverse anemia, in comparison to } \\
\text { darbepoetin alfa }\end{array}$ & (166) \\
\hline \multirow{4}{*}{ GSK1278863 } & $\begin{array}{l}\text { End Stage Renal Disease patients, undergoing } \\
\text { peritoneal dialysis (Phase I, recruiting) }\end{array}$ & Reverse anemia & (167) \\
\hline & $\begin{array}{l}\text { Hyporesponsive rhEPO patients with chronic kidney } \\
\text { disease (Phase II, recruiting) }\end{array}$ & Reverse anemia & (168) \\
\hline & $\begin{array}{l}\text { Diabetic patients with diabetic foot ulcers (Phase I, } \\
\text { recruiting) }\end{array}$ & Treat diabetic foot ulcers & (169) \\
\hline & $\begin{array}{l}\text { Patients undergoing Thoracic Aortic Aneurysm } \\
\text { Repair (Phase II, completed) }\end{array}$ & Reduce Ischemic event & (170) \\
\hline \multirow{8}{*}{$\begin{array}{l}\text { Roxadustat } \\
\text { (FG-4592) }\end{array}$} & $\begin{array}{l}\text { Dialysis-dependent Anemia patients with Chronic } \\
\text { Kidney Disease (Phase III, recruiting) }\end{array}$ & Reverse anemia & (171) \\
\hline & $\begin{array}{l}\text { End Stage Renal Disease Patients in stable Dialysis } \\
\text { (Phase III, recruiting) }\end{array}$ & $\begin{array}{l}\text { Reverse anemia in comparison to } \\
\text { epoetin alfa and darbepoetin alfa }\end{array}$ & (172) \\
\hline & $\begin{array}{l}\text { Anemia patients on stable dialysis (Phase III, } \\
\text { recruiting) }\end{array}$ & $\begin{array}{l}\text { Reverse anemia in comparison to } \\
\text { epoetin alfa (FG-4592) }\end{array}$ & (173) \\
\hline & $\begin{array}{l}\text { Anemia patients with chronic kidney disease not on } \\
\text { dialysis (Phase III, recruiting) }\end{array}$ & Reverse anemia & (174) \\
\hline & $\begin{array}{l}\text { Anemia patients with Chronic Kidney Disease not } \\
\text { requiring Dialysis (Phase III, recruiting), }\end{array}$ & Reverse anemia & (175) \\
\hline & $\begin{array}{l}\text { Anemia patients with End Stage Renal Disease with } \\
\text { newly initiated dialysis (Phase III, recruiting) }\end{array}$ & $\begin{array}{l}\text { Reverse anemia in comparison to } \\
\text { epoetin alfa (FG-4592) }\end{array}$ & (176) \\
\hline & $\begin{array}{l}\text { Anemia patients with Chronic Kidney Disease not on } \\
\text { Dialysis (Phase III, recruiting) }\end{array}$ & Reverse anemia (FG-4592) & (177) \\
\hline & $\begin{array}{l}\text { Anemia patients with Chronic Kidney Disease not on } \\
\text { Dialysis (Phase III, recruiting) }\end{array}$ & $\begin{array}{l}\text { Reverse anemia compared to } \\
\text { darbepoetin alfa }\end{array}$ & (178) \\
\hline
\end{tabular}

Table 2: PHD inhibitors currently undergoing clinical trials (clinicaltrials.gov, 03-02-2016).

Overall, there are implications for PHD inhibition in cardiovascular disease and CVD risk factor management. PHD2 has been extensively studied in cardiovascular disease; however, evidence is sometimes conflicting evidence. Also, the fact that PHD2 deficiency results in embryonic lethality, erythropoiesis and severe phenotypes, makes PHD1 and PHD3 better targets in CVD and other pathologies. Alternatively, cell type-specific PHD2 inhibition might be desirable. 


\section{OVERALL AIM AND HYPOTHESIS}

Current understanding and therapeutic management of atherosclerotic disease has not yielded sufficient reduction in cardiovascular disease events. The aim of this dissertation is to establish the role of hypoxia in atherosclerosis: is hypoxia merely a bystander or causative to atherosclerotic plaque progression and can we define hypoxia as therapeutic target for atherosclerosis?

\section{We hypothesis that plaque hypoxia is causal to atherosclerotic disease progression.}

We will:

1. Reverse plaque hypoxia by enhancing oxygen diffusion to the plaque using carbogen gas (chapter 3).

2. Reduce cellular oxygen demand by dietary nitrate-mediated improvement of mitochondrial efficiency can reverse plaque hypoxia (chapter 4).

3. Interfere with cellular oxygen sensors PHD1 and PHD3 to reverse plaque hypoxia (chapter 5/6).

\section{OUTLINE OF THE DISSERTATION}

Plaque hypoxia mainly co-localizes with macrophages (91). Based on this, we initially screened the literature for recent evidence on hypoxia in atherosclerosis and other inflammatory settings, with particular focus on macrophages (chapter 2).

In chapter 3, the effect of increased oxygen diffusion to murine plaques on atherosclerotic plaque hypoxia and progression was investigated in LDLr deficient mice. Additionally, the underlying mechanism by which hyperoxic therapy could alleviate atherosclerosis was determined in macrophage cultures in vitro.

As opposed to increased oxygen availability, reduction of cellular oxygen consumption was achieved by supplementing LDLr deficient mice with dietary inorganic nitrate in chapter 4. Dietary nitrate has been assigned multiple effects, including blood pressure lowering and anti-inflammatory properties, but also improved mitochondrial respiration efficiency $(105,108,110,111)$, which was hypothesized to reduce cellular oxygen consumption in plaques.

Chapter $\mathbf{5}$ describes the effects of genetic interference with oxygen sensor PHD1 on atherosclerosis development. PHD1 deficient has been shown to reduce cellular oxygen consumption in skeletal muscles (113), and therefore, PHD1 deficient mice backcrossed 
onto a LDLr knockout background were studied. Plaque hypoxia was indeed reversed and plaque development inhibited in PHD1 deficient mice. Surprisingly, PHD1 deficiency also showed pronounced effects on cholesterol metabolism, which were further investigated in vivo in various cholesterol tracing studies.

Pro-inflammatory macrophages mainly express the oxygen sensor PHD3. In chapter 6, the role of hematopoietic and whole body PHD3 deficiency on atherosclerosis development and cholesterol metabolism was then studied. Interestingly, PHD3 interference in both models showed opposite effects as compared to PHD1 deficiency in chapter 5 .

A general discussion of major findings of my dissertation with future perspectives and initial attempts for the making of a PHD1-specific inhibitor are presented in chapter 7. 


\section{REFERENCES}

1. NIH. What Is Atherosclerosis? - NHLBI, NIH [Internet]. 2014 [cited 2016 Feb 5]. Available from: http://www.nhlbi.nih.gov/health/health-topics/topics/atherosclerosis

2. WHO. WHO | Cardiovascular diseases (CVDs) [Internet]. Cardiovascular disaeases. World Health Organization; 2016 [cited 2016 Jan 31]. Available from: http://www.who.int/cardiovascular_ diseases/en/

3. Anitschkow N, Chalatow S. Classics in arteriosclerosis research: On experimental cholesterin steatosis and its significance in the origin of some pathological processes by N. Anitschkow and $\mathrm{S}$. Chalatow, translated by Mary Z. Pelias, 1913. Arteriosclerosis. Jan;3(2):178-82.

4. Clarkson S, Newburgh LH. THE RELATION BETWEEN ATHEROSCLEROSIS AND INGESTED CHOLESTEROL IN THE RABBIT. J Exp Med. 1926 May 30;43(5):595-612.

5. HORLICK L, KATZ LN. The relationship of atheromatosis development in the chicken to the amount of cholesterol added to the diet. Am Heart J. 1949 Oct;38(3):336-49.

6. BEVANS M, KENDALL FE, ABELL LL. Development of atheromatous plaques following production of intimal atherosclerosis by intravenous injection of colloidal cholesterol into rabbits. Am Heart J. 1948 Oct;36(3):473.

7. Reese DM. Fundamentals--Rudolf Virchow and modern medicine. West J Med. 1998 Aug;169(2):105-8.

8. Poston RN, Davies DF. Immunity and inflammation in the pathogenesis of atherosclerosis. Atherosclerosis. 1974 May;19(3):353-67.

9. WAKERLIN GE, MOSS WG, KIELY JP. Effect of experimental renal hypertension on experimental thiouracil-cholesterol atherosclerosis in dogs. Circ Res. 1957 Jul;5(4):426-34.

10. Bailey JM, Butler J. Anti-inflammatory drugs in experimental atherosclerosis. I. Relative potencies for inhibiting plaque formation. Atherosclerosis. Jan;17(3):515-22.

11. ENOS WF, HOLMES RH, BEYER J. Coronary disease among United States soldiers killed in action in Korea; preliminary report. J Am Med Assoc. 1953 Jul 18;152(12):1090-3.

12. Ball RY, Bindman JP, Carpenter KL, Mitchinson MJ. Oxidized low density lipoprotein induces ceroid accumulation by murine peritoneal macrophages in vitro. Atherosclerosis. 1986 May;60(2):17381.

13. Makheja AN, Bloom S, Muesing R, Simon T, Bailey JM. Anti-inflammatory drugs in experimental atherosclerosis. 7. Spontaneous atherosclerosis in WHHL rabbits and inhibition by cortisone acetate. Atherosclerosis. 1989 Apr;76(2-3):155-61.

14. Ross R. Atherosclerosis--an inflammatory disease. N Engl J Med. 1999 Jan 14;340(2):115-

26.

15. Libby P, Ridker PM, Hansson GK. Progress and challenges in translating the biology of atherosclerosis. Nature. Nature Publishing Group; 2011 May 19;473(7347):317-25.

16. Tabas I, Garcia-Cardena G, Owens GK. The cell biology of disease: Recent insights into the cellular biology of atherosclerosis. J Cell Biol. 2015 Apr 13;209(1):13-22.

17. Tabas I, Williams KJ, Borén J. Subendothelial lipoprotein retention as the initiating process in atherosclerosis: update and therapeutic implications. Circulation. 2007 Oct 16;116(16):1832-44.

18. Thorp E, Tabas I. Mechanisms and consequences of efferocytosis in advanced atherosclerosis. J Leukoc Biol. 2009 Nov;86(5):1089-95.

19. Tabas I. Macrophage death and defective inflammation resolution in atherosclerosis. Nat Rev Immunol. 2009/12/05 ed. 2010;10(1):36-46.

20. Stary HC, Chandler AB, Dinsmore RE, Fuster V, Glagov S, Insull W, et al. A Definition of Advanced Types of Atherosclerotic Lesions and a Histological Classification of Atherosclerosis: A Report From the Committee on Vascular Lesions of the Council on Arteriosclerosis, American Heart Association. Circulation. 1995 Sep 1;92(5):1355-74. 
21. Glagov S, Weisenberg E, Zarins CK, Stankunavicius R, Kolettis GJ. Compensatory enlargement of human atherosclerotic coronary arteries. N Engl J Med. 1987 May 28;316(22):1371-5. 22. Richardson PD, Davies MJ, Born G V. Influence of plaque configuration and stress distribution on fissuring of coronary atherosclerotic plaques. Lancet (London, England). 1989 Oct 21;2(8669):941-4.

23. Burke AP, Farb A, Malcom GT, Liang YH, Smialek J, Virmani R. Coronary risk factors and plaque morphology in men with coronary disease who died suddenly. N Engl J Med. 1997 May 1;336(18):1276-82.

24. Virmani R, Burke AP, Farb A, Kolodgie FD. Pathology of the unstable plaque. Prog Cardiovasc Dis. 2002;44(5):349-56.

25. Fernández-Ortiz A, Badimon JJ, Falk E, Fuster V, Meyer B, Mailhac A, et al. Characterization of the relative thrombogenicity of atherosclerotic plaque components: implications for consequences of plaque rupture. J Am Coll Cardiol. 1994 Jun;23(7):1562-9.

26. Lusis AJ. Atherosclerosis. Nature. Macmillan Magazines Ltd.; 2000 Sep 14;407(6801):233-

41.

27. Williams KJ, Feig JE, Fisher EA. Cellular and molecular mechanisms for rapid regression of atherosclerosis: from bench top to potentially achievable clinical goal. Curr Opin Lipidol. 2007 Aug;18(4):443-50.

28. Olofsson SO, Stillemark-Billton P, Asp L. Intracellular assembly of VLDL: two major steps in separate cell compartments. Trends Cardiovasc Med. 2000 Nov;10(8):338-45.

29. Cooper AD. Hepatic uptake of chylomicron remnants. J Lipid Res. 1997 Nov;38(11):2173-

92.

30. Glomset JA. The metabolic role of lecithin: cholesterol acyltransferase: perspectives from pathology. Adv Lipid Res. 1973 Jan;11:1-65.

31. Rader DJ. Regulation of reverse cholesterol transport and clinical implications. Am J Cardiol. 2003 Aug 18;92(4A):42J - 49J.

32. Dietschy JM, Turley SD, Spady DK. Role of liver in the maintenance of cholesterol and low density lipoprotein homeostasis in different animal species, including humans. J Lipid Res. 1993 Oct;34(10):1637-59.

33. Attie AD, Kastelein JP, Hayden MR. Pivotal role of ABCA1 in reverse cholesterol transport influencing HDL levels and susceptibility to atherosclerosis. J Lipid Res. 2001 Nov;42(11):1717-26.

34. Brooks-Wilson A, Marcil M, Clee SM, Zhang LH, Roomp K, van Dam M, et al. Mutations in ABC1 in Tangier disease and familial high-density lipoprotein deficiency. Nat Genet. 1999 Aug;22(4):336-45.

35. Bodzioch M, Orsó E, Klucken J, Langmann T, Böttcher A, Diederich W, et al. The gene encoding ATP-binding cassette transporter 1 is mutated in Tangier disease. Nat Genet. 1999 Aug;22(4):347-51.

36. Rust S, Rosier M, Funke H, Real J, Amoura Z, Piette JC, et al. Tangier disease is caused by mutations in the gene encoding ATP-binding cassette transporter 1. Nat Genet. 1999 Aug;22(4):352-5.

37. Timmins JM, Lee J-Y, Boudyguina E, Kluckman KD, Brunham LR, Mulya A, et al. Targeted inactivation of hepatic Abca1 causes profound hypoalphalipoproteinemia and kidney hypercatabolism of apoA-I. J Clin Invest. 2005 May;115(5):1333-42.

38. Matsuura F, Wang N, Chen W, Jiang X-C, Tall AR. HDL from CETP-deficient subjects shows enhanced ability to promote cholesterol efflux from macrophages in an apoE- and ABCG1dependent pathway. J Clin Invest. 2006 May;116(5):1435-42.

39. Su K, Sabeva NS, Liu J, Wang Y, Bhatnagar S, van der Westhuyzen DR, et al. The ABCG5 ABCG8 sterol transporter opposes the development of fatty liver disease and loss of glycemic control independently of phytosterol accumulation. J Biol Chem. 2012 Aug 17;287(34):28564-75.

40. Yu L, Hammer RE, Li-Hawkins J, Von Bergmann K, Lutjohann D, Cohen JC, et al. 
Disruption of Abcg5 and Abcg8 in mice reveals their crucial role in biliary cholesterol secretion. Proc Natl Acad Sci U S A. 2002 Dec 10;99(25):16237-42.

41. Temel RE, Tang W, Ma Y, Rudel LL, Willingham MC, Ioannou YA, et al. Hepatic Niemann-

Pick C1-like 1 regulates biliary cholesterol concentration and is a target of ezetimibe. J Clin Invest. 2007 Jul;117(7):1968-78.

42. van der Velde AE, Vrins CLJ, van den Oever K, Seemann I, Oude Elferink RPJ, van Eck $\mathrm{M}$, et al. Regulation of direct transintestinal cholesterol excretion in mice. Am J Physiol Gastrointest Liver Physiol. 2008 Jul 1;295(1):G203-8.

43. Brufau G, Kuipers F, Lin Y, Trautwein EA, Groen AK. A reappraisal of the mechanism by which plant sterols promote neutral sterol loss in mice. PLoS One. Public Library of Science; 2011 Jan 30;6(6):e21576.

44. van der Veen JN, van Dijk TH, Vrins CLJ, van Meer H, Havinga R, Bijsterveld K, et al. Activation of the liver $\mathrm{X}$ receptor stimulates trans-intestinal excretion of plasma cholesterol. J Biol Chem. 2009 Jul 17;284(29):19211-9.

45. Vrins CLJ, Ottenhoff R, van den Oever K, de Waart DR, Kruyt JK, Zhao Y, et al. Transintestinal cholesterol efflux is not mediated through high density lipoprotein. J Lipid Res. 2012 Oct;53(10):2017-23.

46. van der Velde AE, Vrins CLJ, van den Oever K, Kunne C, Oude Elferink RPJ, Kuipers F, et al. Direct intestinal cholesterol secretion contributes significantly to total fecal neutral sterol excretion in mice. Gastroenterology. 2007 Sep;133(3):967-75.

47. Le May C, Berger JM, Lespine A, Pillot B, Prieur X, Letessier E, et al. Transintestinal cholesterol excretion is an active metabolic process modulated by PCSK9 and statin involving ABCB1. Arterioscler Thromb Vasc Biol. 2013 Jul 1;33(7):1484-93.

48. Brown JM, Bell TA, Alger HM, Sawyer JK, Smith TL, Kelley K, et al. Targeted depletion of hepatic ACAT2-driven cholesterol esterification reveals a non-biliary route for fecal neutral sterol loss. J Biol Chem. 2008 Apr 18;283(16):10522-34.

49. Stange EF, Dietschy JM. Cholesterol synthesis and low density lipoprotein uptake are regulated independently in rat small intestinal epithelium. Proc Natl Acad Sci U S A. 1983 Sep;80(18):5739-43.

50. García-Miranda P, Peral MJ, Ilundain AA. Rat small intestine expresses the reelinDisabled-1 signalling pathway. Exp Physiol. 2010 Apr;95(4):498-507.

51. Herz J, Hamann U, Rogne S, Myklebost O, Gausepohl H, Stanley KK. Surface location and high affinity for calcium of a 500-kd liver membrane protein closely related to the LDL-receptor suggest a physiological role as lipoprotein receptor. EMBO J. 1988 Dec 20;7(13):4119-27.

52. Hong C, Duit S, Jalonen P, Out R, Scheer L, Sorrentino V, et al. The E3 ubiquitin ligase IDOL induces the degradation of the low density lipoprotein receptor family members VLDLR and ApoER2. J Biol Chem. 2010 Jun 25;285(26):19720-6.

53. Zelcer N, Hong C, Boyadjian R, Tontonoz P. LXR regulates cholesterol uptake through Idol-dependent ubiquitination of the LDL receptor. Science. 2009 Jul 3;325(5936):100-4.

54. Kingwell BA, Chapman MJ, Kontush A, Miller NE. HDL-targeted therapies: progress, failures and future. Nat Rev Drug Discov. Nature Publishing Group, a division of Macmillan Publishers Limited. All Rights Reserved.; 2014 Jun;13(6):445-64.

55. Asztalos BF, Roheim PS, Milani RL, Lefevre M, McNamara JR, Horvath K V., et al. Distribution of ApoA-I-Containing HDL Subpopulations in Patients With Coronary Heart Disease. Arterioscler Thromb Vasc Biol. 2000 Dec 1;20(12):2670-6.

56. Rohatgi A, Khera A, Berry JD, Givens EG, Ayers CR, Wedin KE, et al. HDL cholesterol efflux capacity and incident cardiovascular events. N Engl J Med. 2014 Dec 18;371(25):2383-93.

57. Khera A V, Cuchel M, de la Llera-Moya M, Rodrigues A, Burke MF, Jafri K, et al. Cholesterol efflux capacity, high-density lipoprotein function, and atherosclerosis. N Engl J Med. 
2011 Jan 13;364(2):127-35.

58. Barter PJ, Brewer HB, Chapman MJ, Hennekens CH, Rader DJ, Tall AR. Cholesteryl ester transfer protein: a novel target for raising HDL and inhibiting atherosclerosis. Arterioscler Thromb Vasc Biol. 2003 Feb 1;23(2):160-7.

59. Barter PJ, Caulfield M, Eriksson M, Grundy SM, Kastelein JJP, Komajda M, et al. Effects of torcetrapib in patients at high risk for coronary events. N Engl J Med. 2007 Nov 22;357(21):2109-22.

60. Schwartz GG, Olsson AG, Abt M, Ballantyne CM, Barter PJ, Brumm J, et al. Effects of dalcetrapib in patients with a recent acute coronary syndrome. N Engl J Med. 2012 Nov 29;367(22):2089_ 99.

61. Eli Lilly and Company. Lilly to Discontinue Development of Evacetrapib for High-Risk Atherosclerotic Cardiovascular Disease (NYSE:LLY) [Internet]. 2015 [cited 2016 Feb 12]. Available from: https://investor.lilly.com/releasedetail.cfm?ReleaseID=936130

62. HPS2-THRIVE Collaborative Group. HPS2-THRIVE randomized placebo-controlled trial in 25673 high-risk patients of ER niacin/laropiprant: trial design, pre-specified muscle and liver outcomes, and reasons for stopping study treatment. Eur Heart J. 2013 May 1;34(17):1279-91.

63. Boden WE, Probstfield JL, Anderson T, Chaitman BR, Desvignes-Nickens P, Koprowicz $\mathrm{K}$, et al. Niacin in patients with low HDL cholesterol levels receiving intensive statin therapy. N Engl J Med. 2011 Dec 15;365(24):2255-67.

64. Tardif J-C, Ballantyne CM, Barter P, Dasseux J-L, Fayad ZA, Guertin M-C, et al. Effects of the high-density lipoprotein mimetic agent CER-001 on coronary atherosclerosis in patients with acute coronary syndromes: a randomized trial. Eur Heart J. 2014 Dec 7;35(46):3277-86.

65. Kootte RS, Smits LP, van der Valk FM, Dasseux J-L, Keyserling CH, Barbaras R, et al. Effect of open-label infusion of an apoA-I-containing particle (CER-001) on RCT and artery wall thickness in patients with FHA. J Lipid Res. 2015 Mar 1;56(3):703-12.

66. CDC. National Health Report: Leading Causes of Morbidity and Mortality and Associated Behavioral Risk and Protective Factors—United States, 2005-2013. MMWR. 2014;63(04):3-27.

67. Reiner Ž, De Bacquer D, Kotseva K, Prugger C, De Backer G, Wood D. Treatment potential for dyslipidaemia management in patients with coronary heart disease across Europe: findings from the EUROASPIRE III survey. Atherosclerosis. 2013 Dec;231(2):300-7.

68. Reiner Ž , Tedeschi-Reiner E. Prevalence and types of persistent dyslipidemia in patients treated with statins. Croat Med J. 2013 Aug;54(4):339-45.

69. Fitchett DH, Hegele RA, Verma S. Cardiology patient page. Statin intolerance. Circulation. 2015 Mar 31;131(13):e389-91.

70. Everett BM, Smith RJ, Hiatt WR. Reducing LDL with PCSK9 Inhibitors--The Clinical Benefit of Lipid Drugs. N Engl J Med. 2015 Oct 22;373(17):1588-91.

71. ClinicalTrials.gov. Monoclonal Antibody Against PCSK9 to Reduce Elevated Low-density Lipoprotein Cholesterol(LDL-C) in Adults Currently Not Receiving Drug Therapy for EasingLipidLevels - Study Results - ClinicalTrials.gov [Internet]. [cited 2016 Feb 5]. Available from: https://clinicaltrials. gov/ct2/show $/$ results $/$ NCT01375777?sect=X70156\&term=PCSK9\&rank=16\#outcome1

72. Yasuda T, Grillot D, Billheimer JT, Briand F, Delerive P, Huet S, et al. Tissue-specific liver X receptor activation promotes macrophage reverse cholesterol transport in vivo. Arterioscler Thromb Vasc Biol. 2010 Apr;30(4):781-6.

73. Lo Sasso G, Murzilli S, Salvatore L, D’Errico I, Petruzzelli M, Conca P, et al. Intestinal specific LXR activation stimulates reverse cholesterol transport and protects from atherosclerosis. Cell Metab. Elsevier; 2010 Aug 4;12(2):187-93.

74. Ge L, Wang J, Qi W, Miao H-H, Cao J, Qu Y-X, et al. The cholesterol absorption inhibitor ezetimibe acts by blocking the sterol-induced internalization of NPC1L1. Cell Metab. 2008 Jun;7(6):508-19.

75. Jakulj L, Vissers MN, van Roomen CP, van der Veen JN, Vrins CLJ, Kunne C, et al. Ezetimibe 
stimulates faecal neutral sterol excretion depending on abcg8 function in mice. FEBS Lett. 2010 Aug 20;584(16):3625-8.

76. Cannon CP, Blazing MA, Giugliano RP, McCagg A, White JA, Theroux P, et al. Ezetimibe Added to Statin Therapy after Acute Coronary Syndromes. N Engl J Med. 2015 Jun 3;372(25):2387-97. 77. Grünhage F, Acalovschi M, Tirziu S, Walier M, Wienker TF, Ciocan A, et al. Increased gallstone risk in humans conferred by common variant of hepatic ATP-binding cassette transporter for cholesterol. Hepatology. 2007 Sep;46(3):793-801.

78. Admirand WH, Small DM. The physicochemical basis of cholesterol gallstone formation in man. J Clin Invest. 1968 May;47(5):1043-52.

79. Bäck M, Hansson GK. Anti-inflammatory therapies for atherosclerosis. Nat Rev Cardiol. Nature Publishing Group, a division of Macmillan Publishers Limited. All Rights Reserved.; 2015 Apr;12(4):199-211.

80. Bäck M, Yin L, Ingelsson E. Cyclooxygenase-2 inhibitors and cardiovascular risk in a nationwide cohort study after the withdrawal of rofecoxib. Eur Heart J. 2012 Aug;33(15):1928-33.

81. Nicholls SJ, Kastelein JJP, Schwartz GG, Bash D, Rosenson RS, Cavender MA, et al. Varespladib and cardiovascular events in patients with an acute coronary syndrome: the VISTA-16 randomized clinical trial. JAMA. 2014 Jan 15;311(3):252-62.

82. Tzellos T, Kyrgidis A, Zouboulis CC. Re-evaluation of the risk for major adverse cardiovascular events in patients treated with anti-IL-12/23 biological agents for chronic plaque psoriasis: a metaanalysis of randomized controlled trials. J Eur Acad Dermatol Venereol. 2013 May;27(5):622-7.

83. Vesselinovitch D, Wissler RW, Fisher-Dzoga K, Hughes R, Dubien L. Regression of atherosclerosis in rabbits. I. Treatment with low-fat diet, hyperoxia and hypolipidemic agents. Atherosclerosis. Jan;19(2):259-75.

84. Heughan C, Niinikoski J, Hunt TK. Oxygen tensions in lesions of experimental atherosclerosis of rabbits. Atherosclerosis. 17(3):361-7.

85. Torres Filho IP, Leunig M, Yuan F, Intaglietta M, Jain RK. Noninvasive measurement of microvascular and interstitial oxygen profiles in a human tumor in SCID mice. Proc Natl Acad Sci U S A. 1994 Mar 15;91(6):2081-5.

86. Raleigh JA, Koch CJ. Importance of thiols in the reductive binding of 2-nitroimidazoles to macromolecules. Biochem Pharmacol. 1990 Dec;40(11):2457-64.

87. Carreau A, El Hafny-Rahbi B, Matejuk A, Grillon C, Kieda C. Why is the partial oxygen pressure of human tissues a crucial parameter? Small molecules and hypoxia. J Cell Mol Med. 2011 Jun;15(6):1239-53.

88. van der Valk FM, Sluimer JC, Vöö SA, Verberne HJ, Nederveen AJ, Windhorst AD, et al. In Vivo Imaging of Hypoxia in Atherosclerotic Plaques in Humans. JACC Cardiovasc Imaging. 2015 Nov 5;8(11):1340-1.

89. Parathath S, Mick SL, Feig JE, Joaquin V, Grauer L, Habiel DM, et al. Hypoxia is present in murine atherosclerotic plaques and has multiple adverse effects on macrophage lipid metabolism. Circ Res. 2011/09/17 ed. 2011;109(10):1141-52.

90. Marsch E, Theelen TL, Demandt JAF, Jeurissen M, van Gink M, Verjans R, et al. Reversal of Hypoxia in Murine Atherosclerosis Prevents Necrotic Core Expansion by Enhancing Efferocytosis. Arterioscler Thromb Vasc Biol. 2014 Sep 25;34(12):2545-53.

91. Sluimer JC, Gasc J-M, van Wanroij JL, Kisters N, Groeneweg M, Sollewijn Gelpke MD, et al. Hypoxia, hypoxia-inducible transcription factor, and macrophages in human atherosclerotic plaques are correlated with intraplaque angiogenesis. J Am Coll Cardiol. 2008 Apr 1;51(13):1258-65.

92. NIH. Who Needs Oxygen Therapy? - NHLBI, NIH [Internet]. [cited 2016 Feb 10]. Available from: http://www.nhlbi.nih.gov/health/health-topics/topics/oxt/whoneeds

93. Kudchodkar BJ, Wilson J, Lacko A, Dory L. Hyperbaric oxygen reduces the progression and accelerates the regression of atherosclerosis in rabbits. Arterioscler Thromb Vasc Biol. 2000 
Jun;20(6):1637-43.

94. Kudchodkar BJ, Pierce A, Dory L. Chronic hyperbaric oxygen treatment elicits an antioxidant response and attenuates atherosclerosis in apoE knockout mice. Atherosclerosis. 2007 Jul;193(1):28-35.

95. Kudchodkar B, Jones H, Simecka J, Dory L. Hyperbaric oxygen treatment attenuates the proinflammatory and immune responses in apolipoprotein E knockout mice. Clin Immunol. 2008/07/04 ed. 2008;128(3):435-41.

96. Chen S-Y, Huang E, Wang V, Fan Y-M, Ho C-F, Yip P-K. Improvement of clinical outcome and cerebral perfusion in a patient of atherosclerotic cerebral infarction after repetitive hyperbaric oxygen treatment--a case report and literature review. Undersea Hyperb Med. 2011;38(5):375-9.

97. Tandara AA, Mustoe TA. Oxygen in Wound Healing?More than a Nutrient. World J Surg. 2004 Mar 1;28(3):294-300.

98. Plafki C, Peters P, Almeling M, Welslau W, Busch R. Complications and side effects of hyperbaric oxygen therapy. Aviat Space Environ Med. 2000 Feb;71(2):119-24.

99. Chaplin DJ, Horsman MR, Siemann DW. Further evaluation of nicotinamide and carbogen as a strategy to reoxygenate hypoxic cells in vivo: importance of nicotinamide dose and pre-irradiation breathing time. Br J Cancer. 1993 Aug;68(2):269-73.

100. Yip K, Alonzi R. Carbogen gas and radiotherapy outcomes in prostate cancer. Ther Adv Urol. 2013 Feb;5(1):25-34.

101. Lee H-J, Park CY, Lee JH, Yang H-S, Kim JH, Ban MJ, et al. Therapeutic effects of carbogen inhalation and lipo-prostaglandin E1 in sudden hearing loss. Yonsei Med J. 2012 Sep;53(5):999-1004.

102. Ashkanian M, Borghammer P, Gjedde A, Ostergaard L, Vafaee M. Improvement of brain tissue oxygenation by inhalation of carbogen. Neuroscience. 2008 Oct 28;156(4):932-8.

103. Alonzi R, Padhani AR, Maxwell RJ, Taylor NJ, Stirling JJ, Wilson JI, et al. Carbogen breathing increases prostate cancer oxygenation: a translational MRI study in murine xenografts and humans. $\mathrm{Br}$ J Cancer. 2009 Feb 24;100(4):644-8.

104. Larsen FJ, Schiffer TA, Borniquel S, Sahlin K, Ekblom B, Lundberg JO, et al. Dietary inorganic nitrate improves mitochondrial efficiency in humans. Cell Metab. 2011 Feb 2;13(2):149-59.

105. Bentley R, Gray SR, Schwarzbauer C, Dawson D, Frenneaux M, He J. Dietary nitrate reduces skeletal muscle oxygenation response to physical exercise: a quantitative muscle functional MRI study. Physiol Rep. 2014 Jul 1;2(7).

106. Larsen FJ, Weitzberg E, Lundberg JO, Ekblom B. Effects of dietary nitrate on oxygen cost during exercise. Acta Physiol (Oxf). 2007 Sep;191(1):59-66.

107. Larsen FJ, Weitzberg E, Lundberg JO, Ekblom B. Dietary nitrate reduces maximal oxygen consumption while maintaining work performance in maximal exercise. Free Radic Biol Med. 2010 Jan 15;48(2):342-7.

108. Bryan NS, Calvert JW, Elrod JW, Gundewar S, Ji SY, Lefer DJ. Dietary nitrite supplementation protects against myocardial ischemia-reperfusion injury. Proc Natl Acad Sci U S A. 2007 Nov 27:104(48):19144-9.

109. Larsen FJ, Ekblom B, Sahlin K, Lundberg JO, Weitzberg E. Effects of dietary nitrate on blood pressure in healthy volunteers. N Engl J Med. 2006 Dec 28;355(26):2792-3.

110. Bond V, Curry BH, Adams RG, Millis RM, Haddad GE. Cardiorespiratory function associated with dietary nitrate supplementation. Appl Physiol Nutr Metab. 2014 Feb;39(2):168-72.

111. Khalifi S, Rahimipour A, Jeddi S, Ghanbari M, Kazerouni F, Ghasemi A. Dietary nitrate improves glucose tolerance and lipid profile in an animal model of hyperglycemia. Nitric Oxide. 2014 Nov 18;44C:24-30.

112. Jädert C, Petersson J, Massena S, Ahl D, Grapensparr L, Holm L, et al. Decreased leukocyte recruitment by inorganic nitrate and nitrite in microvascular inflammation and NSAID-induced intestinal injury. Free Radic Biol Med. 2012 Feb 1;52(3):683-92.

113. Aragonés J, Schneider M, Van Geyte K, Fraisl P, Dresselaers T, Mazzone M, et al. Deficiency 
or inhibition of oxygen sensor Phd1 induces hypoxia tolerance by reprogramming basal metabolism. Nat Genet. 2008 Feb;40(2):170-80.

114. Epstein ACR, Gleadle JM, McNeill LA, Hewitson KS, O'Rourke J, Mole DR, et al. C. elegans EGL-9 and Mammalian Homologs Define a Family of Dioxygenases that Regulate HIF by Prolyl Hydroxylation. Cell. 2001 Oct;107(1):43-54.

115. Wang GL, Jiang BH, Rue EA, Semenza GL. Hypoxia-inducible factor 1 is a basic-helixloop-helix-PAS heterodimer regulated by cellular O2 tension. Proc Natl Acad Sci U S A. 1995 Jun 6;92(12):5510-4.

116. Wyatt AW, Steinert JR, Wheeler-Jones CPD, Morgan AJ, Sugden D, Pearson JD, et al. Early activation of the $\mathrm{p} 42 / \mathrm{p} 44 \mathrm{MAPK}$ pathway mediates adenosine-induced nitric oxide production in human endothelial cells: a novel calcium-insensitive mechanism. FASEB J. 2002 Oct 1;16(12):1584-94. 117. Uchida T, Rossignol F, Matthay MA, Mounier R, Couette S, Clottes E, et al. Prolonged hypoxia differentially regulates hypoxia-inducible factor (HIF)-1alpha and HIF-2alpha expression in lung epithelial cells: implication of natural antisense HIF-1alpha. J Biol Chem. 2004 Apr 9;279(15):14871-8.

118. Lin Q, Cong X, Yun Z. Differential hypoxic regulation of hypoxia-inducible factors 1alpha and 2alpha. Mol Cancer Res. 2011 Jun 1;9(6):757-65.

119. Semenza GL. Targeting HIF-1 for cancer therapy. Nat Rev Cancer. 2003 Oct;3(10):721-32. 120. Hu C-J, Wang L-Y, Chodosh LA, Keith B, Simon MC. Differential roles of hypoxiainducible factor 1alpha (HIF-1alpha) and HIF-2alpha in hypoxic gene regulation. Mol Cell Biol. 2003 Dec;23(24):9361-74.

121. Ema M, Taya S, Yokotani N, Sogawa K, Matsuda Y, Fujii-Kuriyama Y. A novel bHLH-PAS factor with close sequence similarity to hypoxia-inducible factor 1 regulates the VEGF expression and is potentially involved in lung and vascular development. Proc Natl Acad Sci. 1997 Apr 29;94(9):42738.

122. Heidbreder M, Fröhlich F, Jöhren O, Dendorfer A, Qadri F, Dominiak P. Hypoxia rapidly activates HIF-3alpha mRNA expression. FASEB J. 2003 Aug;17(11):1541-3.

123. Masson N, Ratcliffe PJ. HIF prolyl and asparaginyl hydroxylases in the biological response to intracellular O(2) levels. J Cell Sci. 2003 Aug 1;116(Pt 15):3041-9.

124. Gerald D, Berra E, Frapart YM, Chan DA, Giaccia AJ, Mansuy D, et al. JunD reduces tumor angiogenesis by protecting cells from oxidative stress. Cell. 2004 Sep 17;118(6):781-94.

125. Pan Y, Mansfield KD, Bertozzi CC, Rudenko V, Chan DA, Giaccia AJ, et al. Multiple factors affecting cellular redox status and energy metabolism modulate hypoxia-inducible factor prolyl hydroxylase activity in vivo and in vitro. Mol Cell Biol. 2007 Feb;27(3):912-25.

126. Selak MA, Armour SM, MacKenzie ED, Boulahbel H, Watson DG, Mansfield KD, et al. Succinate links TCA cycle dysfunction to oncogenesis by inhibiting HIF- $\alpha$ prolyl hydroxylase. Cancer Cell. 2005 Jan; 7(1):77-85.

127. Pescador N, Cuevas Y, Naranjo S, Alcaide M, Villar D, Landázuri MO, et al. Identification of a functional hypoxia-responsive element that regulates the expression of the egl nine homologue 3 (egln3/phd3) gene. Biochem J. 2005 Aug 15;390(Pt 1):189-97.

128. Metzen E, Stiehl DP, Doege K, Marxsen JH, Hellwig-Bürgel T, Jelkmann W. Regulation of the prolyl hydroxylase domain protein 2 (phd2/egln-1) gene: identification of a functional hypoxiaresponsive element. Biochem J. 2005 May 1;387(Pt 3):711-7.

129. Nakayama K, Frew IJ, Hagensen M, Skals M, Habelhah H, Bhoumik A, et al. Siah2 regulates stability of prolyl-hydroxylases, controls HIF1alpha abundance, and modulates physiological responses to hypoxia. Cell. 2004 Jun 25;117(7):941-52.

130. Tuckerman JR, Zhao Y, Hewitson KS, Tian Y-M, Pugh CW, Ratcliffe PJ, et al. Determination and comparison of specific activity of the HIF-prolyl hydroxylases. FEBS Lett. 2004 Oct 8;576(12):145-50.

131. Huang J, Zhao Q, Mooney SM, Lee FS. Sequence determinants in hypoxia-inducible factor- 
1alpha for hydroxylation by the prolyl hydroxylases PHD1, PHD2, and PHD3. J Biol Chem. 2002 Oct 18;277(42):39792-800.

132. Appelhoff RJ, Tian Y-M, Raval RR, Turley H, Harris AL, Pugh CW, et al. Differential function of the prolyl hydroxylases PHD1, PHD2, and PHD3 in the regulation of hypoxia-inducible factor. J Biol Chem. 2004 Sep 10;279(37):38458-65.

133. Berra E, Roux D, Richard DE, Pouysségur J. Hypoxia-inducible factor-1 alpha (HIF-1 alpha) escapes $\mathrm{O}(2)$-driven proteasomal degradation irrespective of its subcellular localization: nucleus or cytoplasm. EMBO Rep. 2001 Jul;2(7):615-20.

134. Cummins EP, Berra E, Comerford KM, Ginouves A, Fitzgerald KT, Seeballuck F, et al. Prolyl hydroxylase-1 negatively regulates IkappaB kinase-beta, giving insight into hypoxia-induced NFkappaB activity. Proc Natl Acad Sci U S A. 2006 Nov 28;103(48):18154-9.

135. Zheng X, Zhai B, Koivunen P, Shin SJ, Lu G, Liu J, et al. Prolyl hydroxylation by EgIN2 destabilizes FOXO3a by blocking its interaction with the USP9x deubiquitinase. Genes Dev. 2014 Jul 1;28(13):1429-44.

136. Köditz J, Nesper J, Wottawa M, Stiehl DP, Camenisch G, Franke C, et al. Oxygen-dependent ATF-4 stability is mediated by the PHD3 oxygen sensor. Blood. American Society of Hematology; 2007 Nov 15;110(10):3610-7.

137. Xie L, Xiao K, Whalen EJ, Forrester MT, Freeman RS, Fong G, et al. Oxygen-regulated beta(2)-adrenergic receptor hydroxylation by EGLN3 and ubiquitylation by pVHL. Sci Signal. 2009 Jan 7;2(78):ra33.

138. Luo W, Hu H, Chang R, Zhong J, Knabel M, O’Meally R, et al. Pyruvate kinase M2 is a PHD3stimulated coactivator for hypoxia-inducible factor 1. Cell. Elsevier; 2011 May 27;145(5):732-44.

139. Eckle T, Köhler D, Lehmann R, El Kasmi K, Eltzschig HK. Hypoxia-inducible factor-1 is central to cardioprotection: a new paradigm for ischemic preconditioning. Circulation. $2008 \mathrm{Jul}$ 8;118(2):166-75.

140. Ockaili R, Natarajan R, Salloum F, Fisher BJ, Jones D, Fowler AA, et al. HIF-1 activation attenuates postischemic myocardial injury: role for heme oxygenase-1 in modulating microvascular chemokine generation. Am J Physiol Heart Circ Physiol. 2005 Aug 1;289(2):H542-8.

141. Vogler M, Zieseniss A, Hesse AR, Levent E, Tiburcy M, Heinze E, et al. Pre- and postconditional inhibition of prolyl-4-hydroxylase domain enzymes protects the heart from an ischemic insult. Pflügers Arch Eur J Physiol. 2015 Oct;467(10):2141-9.

142. Rohrbach S, Simm A, Pregla R, Franke C, Katschinski DM. Age-dependent increase of prolyl-4-hydroxylase domain (PHD) 3 expression in human and mouse heart. Biogerontology. 2005 Jan;6(3):165-71.

143. Lieb ME, Menzies K, Moschella MC, Ni R, Taubman MB. Mammalian EGLN genes have distinct patterns of mRNA expression and regulation. Biochem Cell Biol. 2002 Jan;80(4):421-6.

144. Willam C, Maxwell PH, Nichols L, Lygate C, Tian YM, Bernhardt W, et al. HIF prolyl hydroxylases in the rat; organ distribution and changes in expression following hypoxia and coronary artery ligation. J Mol Cell Cardiol. 2006 Jul;41(1):68-77.

145. Hölscher M, Silter M, Krull S, von Ahlen M, Hesse A, Schwartz P, et al. Cardiomyocytespecific prolyl-4-hydroxylase domain 2 knock out protects from acute myocardial ischemic injury. J Biol Chem. 2011 Apr 1;286(13):11185-94.

146. Natarajan R, Salloum FN, Fisher BJ, Kukreja RC, Fowler AA. Hypoxia inducible factor-1 activation by prolyl 4-hydroxylase-2 gene silencing attenuates myocardial ischemia reperfusion injury. Circ Res. 2006 Jan 6;98(1):133-40.

147. Chen R-L, Nagel S, Papadakis M, Bishop T, Pollard P, Ratcliffe PJ, et al. Roles of individual prolyl-4-hydroxylase isoforms in the first 24 hours following transient focal cerebral ischaemia: insights from genetically modified mice. J Physiol. 2012 Aug 15;590(Pt 16):4079-91.

148. Karsikas S, Myllymäki M, Heikkilä M, Sormunen R, Kivirikko KI, Myllyharju J, et al. HIF- 
P4H-2 deficiency protects against skeletal muscle ischemia-reperfusion injury. J Mol Med (Berl). 2015 Oct 10;

149. Loinard C, Ginouvès A, Vilar J, Cochain C, Zouggari Y, Recalde A, et al. Inhibition of prolyl hydroxylase domain proteins promotes therapeutic revascularization. Circulation. 2009 Jul 7;120(1):50 9 .

150. Takeda Y, Costa S, Delamarre E, Roncal C, Leite de Oliveira R, Squadrito ML, et al. Macrophage skewing by Phd 2 haplodeficiency prevents ischaemia by inducing arteriogenesis. Nature. 2011 Nov 3;479(7371):122-6.

151. Rishi MT, Selvaraju V, Thirunavukkarasu M, Shaikh IA, Takeda K, Fong G-H, et al. Deletion of prolyl hydroxylase domain proteins (PHD1, PHD3) stabilizes hypoxia inducible factor-1 alpha, promotes neovascularization, and improves perfusion in a murine model of hind-limb ischemia. Microvasc Res. 2015 Jan; 97:181-8.

152. Adluri RS, Thirunavukkarasu M, Dunna NR, Zhan L, Oriowo B, Takeda K, et al. Disruption of hypoxia-inducible transcription factor-prolyl hydroxylase domain-1 (PHD-1-/-) attenuates ex vivo myocardial ischemia/reperfusion injury through hypoxia-inducible factor- $1 \alpha$ transcription factor and its target genes in mice. Antioxid Redox Signal. 2011 Oct 1;15(7):1789-97.

153. Quaegebeur A, Segura I, Schmieder R, Verdegem D, Decimo I, Bifari F, et al. Deletion or Inhibition of the Oxygen Sensor PHD1 Protects against Ischemic Stroke via Reprogramming of Neuronal Metabolism. Cell Metab. 2016 Jan 6;

154. Schneider M, Van Geyte K, Fraisl P, Kiss J, Aragonés J, Mazzone M, et al. Loss or silencing of the PHD1 prolyl hydroxylase protects livers of mice against ischemia/reperfusion injury. Gastroenterology. 2010 Mar;138(3):1143-54.e1-2.

155. Oriowo B, Thirunavukkarasu M, Selvaraju V, Adluri RS, Zhan L, Takeda K, et al. Targeted gene deletion of prolyl hydroxylase domain protein 3 triggers angiogenesis and preserves cardiac function by stabilizing hypoxia inducible factor 1 alpha following myocardial infarction. Curr Pharm Des. 2014 Jan;20(9):1305-10.

156. Zieseniss A, Hesse AR, Jatho A, Krull S, Hölscher M, Vogel S, et al. Cardiomyocyte-Specific Transgenic Expression of Prolyl-4-Hydroxylase Domain 3 Impairs the Myocardial Response to Ischemia. Cell Physiol Biochem. Karger Publishers; 2015 Jan 27;36(3):843-51.

157. Moslehi J, Minamishima YA, Shi J, Neuberg D, Charytan DM, Padera RF, et al. Loss of hypoxia-inducible factor prolyl hydroxylase activity in cardiomyocytes phenocopies ischemic cardiomyopathy. Circulation. 2010 Sep 7;122(10):1004-16.

158. Minamishima YA, Moslehi J, Bardeesy N, Cullen D, Bronson RT, Kaelin WG. Somatic inactivation of the PHD2 prolyl hydroxylase causes polycythemia and congestive heart failure. Blood. American Society of Hematology; 2008 Mar 15;111(6):3236-44.

159. Takeda K, Aguila HL, Parikh NS, Li X, Lamothe K, Duan L-J, et al. Regulation of adult erythropoiesis by prolyl hydroxylase domain proteins. Blood. 2008 Mar 15;111(6):3229-35.

160. Holliger P, Prospero T, Winter G. "Diabodies": small bivalent and bispecific antibody fragments. Proc Natl Acad Sci U S A. 1993;90(14):6444-8.

161. Musallam KM, Porter JB, Sfeir PM, Tamim HM, Richards T, Lotta LA, et al. Raised haematocrit concentration and the risk of death and vascular complications after major surgery. Br J Surg. 2013 Jul;100(8):1030-6.

162. Phrommintikul A, Haas SJ, Elsik M, Krum H. Mortality and target haemoglobin concentrations in anaemic patients with chronic kidney disease treated with erythropoietin: a metaanalysis. Lancet. Elsevier; 2007 Feb 3;369(9559):381-8.

163. Fraisl P, Aragonés J, Carmeliet P. Inhibition of oxygen sensors as a therapeutic strategy for ischaemic and inflammatory disease. Nat Rev Drug Discov. Nature Publishing Group; 2009 Feb;8(2):139-52.

164. ClinicalTrials.gov. Efficacy and Safety Study to Evaluate Vadadustat for the Correction of 
Anemia in Subjects With Non-dialysis-dependent Chronic Kidney Disease (NDD-CKD) - Full Text View - ClinicalTrials.gov [Internet]. [cited 2016 Feb 3]. Available from: https://clinicaltrials.gov/ct2/ show $/$ NCT02648347?term $=$ HIF + prolyl + hydroxylase\&rank $=4$

165. ClinicalTrials.gov. Study to Assess the Pharmacokinetics of GSK1278863 in Subjects With End Stage Renal Disease Undergoing Peritoneal Dialysis - Full Text View - ClinicalTrials. gov [Internet]. [cited 2016 Feb 3]. Available from: https://clinicaltrials.gov/ct2/show/ NCT02243306?term $=$ HIF + prolyl + hydroxylase\&rank $=7$

166. GSK clincial study Register. GSK Clinical Study Register - Study 114837 [Internet]. [cited 2016 Feb 3]. Available from: http://www.gsk-clinicalstudyregister.com/study/114837\#ps

167. GSK clincial study Register. GSK Clinical Study Register - Study 115760 [Internet]. [cited 2016 Feb 3]. Available from: http://www.gsk-clinicalstudyregister.com/study/115760\#ps

168. GSK clincial study Register. GSK Clinical Study Register - Study 116097 [Internet]. [cited 2016 Feb 3]. Available from: http://www.gsk-clinicalstudyregister.com/study/116097\#ps

169. ClinicalTrials.gov. Safety and Efficacy Study of Roxadustat to Treat Anemia in Patients With Chronic Kidney Disease, on Dialysis - Full Text View - ClinicalTrials.gov [Internet]. [cited 2016 Feb 3]. Available from: https://clinicaltrials.gov/ct2/show/NCT02174731?term=roxadustat\&rank=1

170. ClinicalTrials.gov. Roxadustat in the Treatment of Anemia in End Stage Renal Disease (ESRD) Patients on Stable Dialysis - Full Text View - ClinicalTrials.gov [Internet]. [cited 2016 Feb 3]. Available from: https://clinicaltrials.gov/ct2/show/NCT02278341?term=roxadustat\&rank $=2$

171. ClinicalTrials.gov. Evaluation of Efficacy and Safety of Roxadustat in the Treatment of Anemia in Stable Dialysis Subjects - Full Text View - ClinicalTrials.gov [Internet]. [cited 2016 Feb 3]. Available from: https://clinicaltrials.gov/ct2/show/NCT02273726?term=roxadustat\&rank=3

172. ClinicalTrials.gov. Safety and Efficacy Study of Roxadustat to Treat Anemia in Patients With Chronic Kidney Disease (CKD), Noton Dialysis. -Full TextView-ClinicalTrials.gov [Internet]. [cited 2016 Feb 3]. Available from: https://clinicaltrials.gov/ct2/show/NCT02174627?term=roxadustat\&rank=4 173. ClinicalTrials.gov. Roxadustat in the Treatment of Anemia in Chronic Kidney Disease Patients Not Requiring Dialysis - Full Text View - ClinicalTrials.gov [Internet]. [cited 2016 Feb 3]. Available from: https://clinicaltrials.gov/ct2/show/NCT01887600?term=roxadustat\&rank $=5$

174. ClinicalTrials.gov. Safety and Efficacy Study for Treatment of Anemia in ESRD Newly Initiated Dialysis Patients - Full Text View - ClinicalTrials.gov [Internet]. [cited 2016 Feb 3]. Available from: https:/ clinicaltrials.gov/ct2/show/NCT02052310?term=roxadustat\&rank $=6$

175. ClinicalTrials.gov. A Study of FG-4592 for the Treatment of Anemia in Chronic Kidney Disease Patients Not Receiving Dialysis - Full Text View - ClinicalTrials.gov [Internet]. [cited 2016 Feb 3]. Available from: https://clinicaltrials.gov/ct2/show/NCT01750190?term=roxadustat\&rank=7

176. ClinicalTrials.gov. FG-4592 in the Treatment of Anemia in Chronic Kidney Disease (CKD) Patients, Not on Dialysis, in Comparison to Darbepoetin Alfa - Full Text View ClinicalTrials.gov [Internet]. [cited 2016 Feb 3]. Available from: https://clinicaltrials.gov/ct2/show/ NCT02021318?term $=$ roxadustat\&rank $=8$ 


hypoxia IN athergsclerogIS aND INFLammation

Margch e, gluIMER JC, daEMEN MuAP CURR OPIN LIPIOOL. 2019 OCT;24)(5):999-400. 



\section{ABSTRACT}

\section{PURPOSE OF THE REVIEW}

Hypoxia triggers various cellular processes, both in physiological and pathological conditions and has recently also been implicated in atherosclerosis. This review summarizes the recent evidence for the presence and the role of hypoxia in atherosclerosis. Additionally, it will elucidate on hypoxic signaling, which is interlinked with inflammatory signaling and discuss recent advances in imaging of hypoxia in atherosclerosis.

\section{RECENT FINDINGS}

Hypoxia is present in atherosclerotic plaques in humans and animal models and systemic hypoxia promotes atherosclerosis. Hypoxia stimulates pro-atherosclerotic processes, like deficient lipid efflux, inflammation, interference with macrophage polarization and glucose metabolism. However, the molecular mechanism of hypoxia-mediated atherogenesis remains unclear.

Non-invasive imaging directly targeting plaque hypoxia has been applied in animal models of atherosclerosis, but remains to be validated in humans. Meanwhile, the metabolic marker ${ }^{18} \mathrm{~F}-\mathrm{FDG}$, used to detect human atherosclerosis in vivo, may serve as indirect marker of plaque hypoxia due to enhanced glucose uptake in anaerobic metabolism.

\section{SUMMARY}

Recent studies underscore the pro-atherogenic role of hypoxia in macrophage lipid and glucose metabolism, inflammation and polarization. These studies provide new insights into the pathogenesis of atherosclerosis and unravel novel therapeutic targets and new options for non-invasive imaging of human atherosclerotic plaques. 


\section{EVIDENCE OF hYPOXIA IN ATHEROSCLEROSIS - A HISTORICAL VIEW}

Low oxygen tension, hypoxia, is an important stimulus of both pathological and physiological processes including angiogenesis, inflammation, metabolism and apoptosis. All of these processes are implicated in atherogenesis. Arterial wall hypoxia has been extensively studied using microelectrodes in vitro and in vivo in healthy arteries and atherosclerosis injury-models in rabbits (1-5). More recently, Björnheden et al. $(6,7)$ showed zones of hypoxia in rabbit atherosclerotic plaques, using the imidazole derivate 7-(4'-(2-nitroimidazol-1-yl)-butyl)-theophylline (NITP), and suggested a co-localization of hypoxia with foam cells (macrophages). NITP undergoes intracellular nitroreduction (mainly by cytochrome p450) (8), resulting in reactive intermediates. In cells with an oxygen concentration below $14 \mathrm{mM}$ (equivalent to $10 \mathrm{mmHg}$ at $37^{\circ} \mathrm{C}$ ), these intermediates form stable adducts with cellular thiol groups in proteins, peptides and amino acids (9).

Using the imidazole derivate pimonidazole, we recently conclusively demonstrated the presence of hypoxia in human atherosclerotic plaques in vivo. As for rabbit atherosclerosis, macrophages are the major cell type in human plaques that display signs hypoxia, with co-existent expression of hypoxia-inducible factor $1 \alpha(\mathrm{HIF}-1 \alpha)$ and vascular endothelial growth factor (VEGF). Furthermore, hypoxia correlated with intra-plaque angiogenesis (10). Autoradiography of the imidazole marker $\left[{ }^{18} \mathrm{~F}\right]-\mathrm{EF} 5$ (2-(2-Nitro-1H-imidazol-1yl)-N-(2,2,3,3,3-pentafluoropropyl)), was also able to detect hypoxia in atherosclerotic

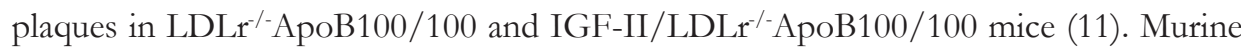
plaque hypoxia, was also confirmed in $\mathrm{LDLr}^{-/}$mice in vivo using pimonidazole, and again co-localized with macrophages (12). Based on these studies, the presence of hypoxia in human and murine atherosclerotic plaques is nowadays widely accepted; however the functional involvement of hypoxia in atherogenesis remains unclear.

Cells respond to hypoxic conditions by adjusting metabolism, angiogenesis, inflammation, migration, endothelial dysfunction and cell survival signaling (13-15). These processes mainly involve the transcription factor HIF-1 (16). HIF-1 is composed of an $\alpha$ and $\beta$ subunit, with HIF- $1 \alpha$ being continuously degraded under normoxic conditions, while hypoxia increases its stability and transcriptional activity (17). In human atherosclerotic plaques, we showed co-expression of the hypoxic marker pimonidazole and HIF-1 $\alpha$, suggesting a role of HIF-1 $\alpha$ in atherosclerosis (10). However, it should be noted that HIF-1 $\alpha$ can also be stabilized under normoxic conditions and therefore does not exclusively represent hypoxia. Atherosclerotic factors including ROS (18), thrombin (19), LPS (20), oxidized LDL (oxLDL) (21), PKC and PI-3K pathways (22) have also been shown to promote HIF-1 $\alpha$ stabilization in normoxia. 
In this review, we will summarize the recent findings on a causal role for hypoxia-driven signaling in atherogenesis. As macrophages present the main hypoxic component of atherosclerotic plaques, we will focus on oxygen deprivation-mediated disturbances in macrophage functions, such as lipid and glucose metabolism, polarization and inflammatory signaling.

\section{ATHEROGENESIS: HYPOXIA-DRIVEN?}

Atherosclerotic vascular disease is the leading cause of morbidity and mortality in the industrialized world (23). Evidence for a clinically relevant and progressive role of hypoxia in atherosclerosis is provided by obstructive sleep apnea (OSA) patients. OSA patients suffer from chronic intermittent cycles of hypoxia and re-oxygenation (CIH) (24) and present with an increased risk for atherosclerosis and subsequent cardiovascular disease (25-30).

$\mathrm{CIH}$ is believed to be the major cause of OSA-associated atherosclerotic cardiac events (reviewed in (31)), in parts, by CIH-induced oxidative stress, which is elevated in OSA patients (32). Even though, local plaque hypoxia has not been shown in OSA patients so far, $\mathrm{CIH}$ models suggest a pro-atherogenic role of systemic hypoxia in atherosclerosis. $\mathrm{CIH}$ induces atherosclerosis in C57B16 mice fed a high cholesterol diet, while C57B16 mice subjected to high cholesterol diet alone did not show lesion development. Exposure of $\mathrm{ApoE}^{-/-}$mice to $\mathrm{CIH}$ accelerated atherosclerosis progression on high cholesterol diet but also on normal chow (33-36). Conversely, early signs of atherosclerosis, including arterial wall thickening and stiffness in OSA patients can be reversed by continuous positive airway pressure therapy (cPAP) $(37,38)$. CPAP reverses upper airway narrowing by delivering compressed air, making unobstructed breathing possible and restoring hypopneas and apneas. Additionally, hyperbaric oxygen treatment (HBOT, 2.4-2.5 atm, $100 \% \mathrm{O}_{2}$ ) reduced atherosclerosis in both rabbit and mouse models. It increased antioxidant enzymes and attenuated both lipid oxidation and the proinflammatory immune response (39-41). In humans, HBOT improved atherosclerosis in patients with diabetic feet and in a case report on reperfusion of atherosclerotic cerebral infarction $(42,43)$. Unfirer et al. proposed that the advantageous effect of HBOT on regression of atherosclerosis and diabetes mellitus, might be due to a reoxygenation-mediated restoration of endothelial function (44). However, the mechanism underlying the beneficial effect of systemic re-oxygenation on atherosclerosis and the effect on plaque hypoxia remains unclear. Moreover, the direct link between HBOT and atherosclerosis regression, as shown in animal models and case reports, remains to be confirmed for large scale human atherosclerosis studies. 
In conclusion, hypoxia is present in atherosclerotic plaques and systemic oxygen alterations in $\mathrm{CIH}$ and HBOT suggest a pro-atherosclerotic effector role of systemic hypoxia. However, if this effect is a mediated by local changes in plaque hypoxia and the underlying molecular mechanism remain obscure.

\section{HYPOXIA AND INFLAMMATION SHARE PATHWAYS: IS NFKB THE KEY?}

Oxygen demand, and thus hypoxia, is particularly elevated at sites of inflammation, e.g. wounds or atherosclerotic lesions (45). In this respect, it is not surprising that hypoxia and inflammatory responses share intracellular pathways, including metabolic alterations, macrophage phenotype switching and oxidative stress signaling. In this review, we will discuss the recent advances in hypoxia and inflammation signaling in atherosclerosis.

Hypoxia has mainly been studied with respect to lipid metabolism, as LDL modification and accumulation in the vessel wall and foam cell formation present critical steps in atherogenesis. Hypoxia promotes triglyceride synthesis and loading of foam cells $(46,47)$, inhibits cholesterol influx and enhances LDL affinity of macrophages (48) (extensively reviewed in (49)). Along this line, foam cell formation could be inhibited in vitro by HIF-1 $\alpha$ RNA interference (50). Mechanistically, the dyslipidemic effect of hypoxia has been linked with LXR expression and down-regulation of cholesterol efflux receptors, such as ABCA-1 (49,51). In fact, HIF-1 $\alpha$ over-expression reduced cholesterol efflux from macrophages, which was even further decreased upon hypoxia (51). A recent study now showed that another inflammatory transcription factor, $\mathrm{NF} \varkappa \mathrm{B}$, was required for cholesterol efflux/uptake receptors $\mathrm{ABCA1}$ and SR-B1 protein expression in $\mathrm{CIH}$-induced atherosclerosis. Additionally, $\mathrm{NF} \varkappa \mathrm{B}$ subunit $\mathrm{p} 50^{-/}$mice presented with reduced hyperlipidemia and foam cell formation in $\mathrm{CIH}$-induced atherosclerosis (52). This confirms earlier findings, where $\mathrm{p} 50^{-/}$attenuated atherosclerosis development in mice and reduced oxLDL uptake by $\mathrm{p} 50^{-/}$macrophages, also in the absence of systemic hypoxia (53). Another study showed that the hypoxic effect on lipid clearance was mediated by inhibition of lipoprotein lipase $(\mathrm{LpL})$ and that this effect was independent of $\mathrm{NF} x \mathrm{~B}$ (54). In addition to the $\mathrm{NF} x \mathrm{~B}$-mediated dyslipidemic effects, $\mathrm{NF} x \mathrm{~B}$ contributed to macrophage differentiation, recruitment and foam cell formation (55), as well as endothelial dysfunction in chronic intermittent hypoxia models (56). 
In conclusion, hypoxia and inflammation share signaling pathways, amongst others $\mathrm{NF} \varkappa \mathrm{B}$ activation. In concert, hypoxia and inflammation affect multiple processes, including lipid metabolism and cholesterol efflux, endothelial dysfunction and inflammation.

\section{hypoxia alters gluCOse metabolism and oxidative gtregs gignaling and VIGE VERSA}

Chronic inflammation is a feature of atherosclerosis and mounting an inflammatory response is an energy-intensive process. At sites of inflammation, macrophages rapidly switch from a resting to an activated state, resulting in increased cytokine production, enhanced phagocytosis and antigen presentation, all resulting in excessive ATP consumption (57). This inflammatory switch has been shown to be at least partially mediated by HIF-1 $\alpha$ in both normoxic and hypoxic conditions $(58,59)$ and is accompanied by a metabolic shift towards glycolysis; a phenomenon known as the Warburg Effect. The Warburg effect describes the switch from oxygen-dependent mitochondria-mediated oxidative phosphorylation (OXPHOS) to glycolysis in tumor cells and highly proliferative cells. This switch can occur both in normoxic and hypoxic conditions and thus involves both aerobic and anaerobic glycolysis $(60,61)$. It is well established that aerobic metabolism via OXPHOS produces more ATP per glucose molecule (in fact 36 ATP) than glycolysis. Yet, cancer and inflammatory cells decide for a seemingly less efficient metabolism via glycolysis, with only 2 ATP being produced per glucose molecule. This discrepancy can be explained by looking at metabolism as an interlinked network, rather than pathways. During glycolysis, precursors of amino acid synthesis and nucleotide anabolism are generated, which are crucial for biomass accumulation and proliferation (61). If nutrients are abundant, cells are hence easily capable to maintain homeostasis by relying purely on glycolysis, both in inflammation and hypoxia.

Upon inflammation and hypoxia, $\mathrm{T}$ cells and macrophages switch their metabolism towards glycolysis, with macrophages presenting with a more pro-inflammatory M1-like phenotype (62-64). In fact, Folco et al. recently demonstrated that hypoxia rather than inflammation promotes glucose uptake by macrophages in vitro (65). M2 macrophages were shown to maintain oxidative phosphorylation for ATP production (reviewed in (66)). Also in rabbit atherosclerotic lesions, hypoxic macrophages showed increased glucose consumption and ATP depletion, suggesting the Warburg effect in atherosclerosis. In vitro, hyperoxic re-oxygenation of the rabbit plaques could reverse ATP depletion, whereas normoxic conditions could not, suggesting that oxygen availability rather than glucose concentration is the limiting factor in ATP production within the plaques (67). In turn, increased glycolysis promotes lactate production and acidification of the plaque environment. In fact, elevated blood lactate has recently been associated with increased 
carotid atherosclerotic wall thickness in humans, although this was partially related to insulin resistance (68). As plaque and systemic acidification are pro-atherogenic $(69,70)$, enhanced lactate due to increased glycolysis may contribute to atherogenesis.

Apart from enhanced lactate production, hypoxia results in increased ROS production by a dys-regulated mitochondrial respiratory chain (reviewed in (71)). ROS are implicated in oxLDL formation, endothelial activation, monocyte-derived macrophage recruitment, activation and death, vascular smooth muscle cell proliferation and death, and matrix remodeling (72,73). Mitochondria, which are majorly dependent on oxygen during respiration, sense oxygen depletion and subsequently release ROS into the cytosol. ROS in turn stabilizes HIF- $1 \alpha$ via various pathways, including inhibition of PHD-mediated HIF hydroxylation (reviewed in (74)). Macrophages, representing the largest hypoxic fraction in atherosclerosis (10), are also the main source of ROS and oxidative stress in the plaque (75), suggesting a link between hypoxia and ROS in plaque progression. In accordance, ROS inhibition by statins has been shown to improve hypoxia-induced endothelial dysfunction (76). Also, OSA patients present with elevated oxidative stress levels and ROS-mediated endothelial dysfunction (37,77-80). These data confirm a metabolic switch towards glycolysis in atherosclerotic macrophages, with subsequent lactate production and ROS production contributing to endothelial dysfunction and plaque advancement. However, whether hypoxia promotes this glycolytic shift, or whether hypoxia is secondary to enhanced glycolysis and inflammation in vivo, remains to be elucidated. Based on the fact that even small mouse lesions present hypoxic areas, we postulated, that macrophage oxygen demand rather than impaired oxygen diffusion promotes hypoxia in macrophages in atherosclerotic plaques (10). This would suggest that plaque hypoxia is secondary to a metabolic switch towards glycolysis during atherosclerosis (see Figure 1). 


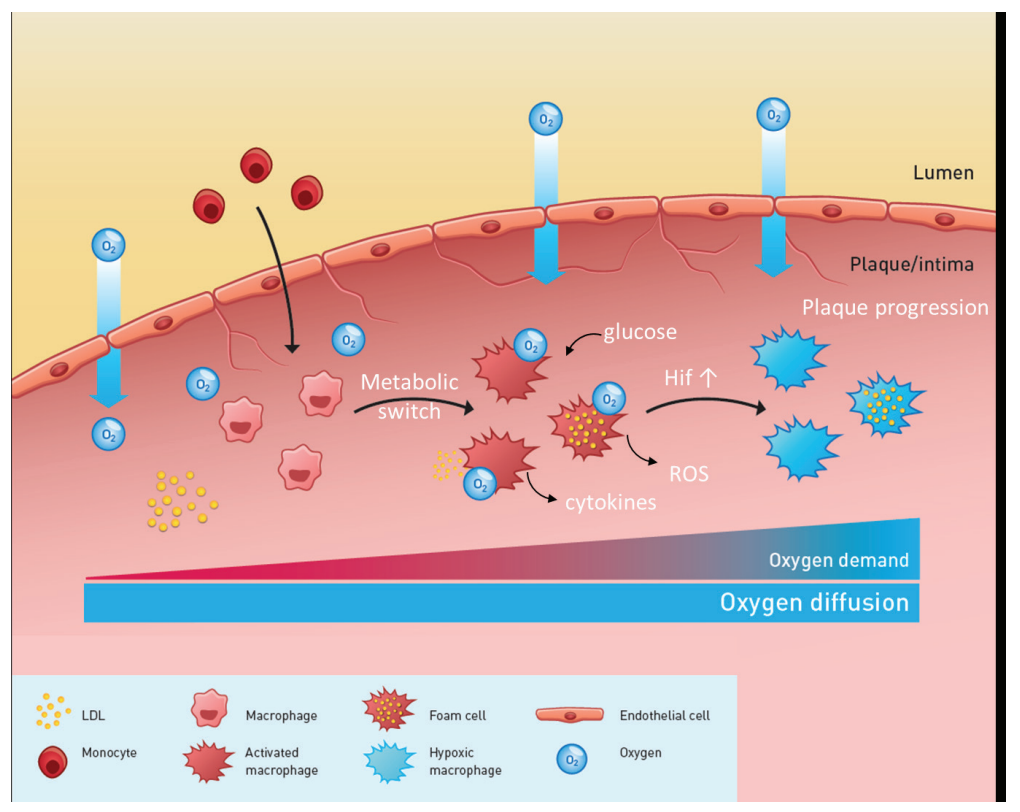

Figure 1: Suggested model of plaque oxygen availability and metabolic effects on macrophages.

Upon entering the arterial wall, monocytes differentiate into macrophages, which in turn take up oxLDL and become activated. With increased inflammation, cytokine and ROS production as well as glucose and ATP demand, macrophage oxygen consumption exceeds oxygen availability. Consequenty, macrophages display signs of hypoxia, even though oxygen diffusion rates from the lumen into the plaque remain constant (credit to Volker Zerbe, wnw.volker-zerbe.de).

\section{hYPOXIA aLters MaCROPHAGE POLARIZATION AND INFLAMMATION}

As indicated, hypoxia can induce the glycolytic switch and M1 macrophages show increased glycolytic activity (64). It has therefore been speculated that hypoxia can promote pro-inflammatory M1 macrophage differentiation thereby aggravating atherogenesis. However, there is conflicting evidence for a hypoxia-mediated M1 macrophage phenotype switch. In vitro, Macrophages display M1 polarization upon hypoxia (81) and HIF-1 $\alpha$ deficient macrophages present a M2 TAM (tumor-associated macrophage) marker profile (82). However, in vivo, M2 TAMs accumulate in hypoxic tumor regions and both M1 and M2 ATMs (adipose tissue macrophages), amass in distinct hypoxic adipose tissue $(81,82)$. These data suggest the presence of an additional M2 macrophage trigger over-riding hypoxia in inflammatory tissue in vivo. In this respect, lactate and $\mathrm{pH}$ as well as growth factors and necrotic debris have been described for the tumor environment (83), suggesting a secondary effect of the Warburg effect and lactate production on M2 polarization. 
In atherosclerotic lesions, both, M1 and M2 macrophage populations increased during plaque progression towards unstable lesions in vivo, with M1 macrophages localizing in rupture prone areas (84). Also studies on tumor hypoxia and macrophage distribution show that mainly M2-like TAMs are localized in hypoxic regions (85), supporting the idea of an additional trigger towards M2-like macrophage marker expression over-riding hypoxia. This is not surprising, as the plaque and tumor environment are very heterogeneous and contain various growth factors and inflammatory mediators. In this respect, we suggest that atherosclerotic plaque macrophages might resemble the tumor macrophages.

In conclusion, hypoxia seems to induce pro-inflammatory responses in macrophages. However, hypoxia is not sufficient in inducing macrophage phenotype switching in vivo, as it is balanced by yet unknown factors.

\section{HYPOXIA AS A NON-INVASIVE IMAgING TOOL OF ATHEROSCLEROSIS}

Knowing the pro-atherosclerotic role of hypoxia, imaging of plaque hypoxia may become a relevant and desirable prognostic and diagnostic tool for atherosclerosis. Several imidazole analogues $\left[{ }^{18} \mathrm{~F}\right]-\mathrm{EF} 5(86),\left[{ }^{18} \mathrm{~F}\right]-\mathrm{HX} 4\left(3-\left[{ }^{18} \mathrm{~F}\right]\right.$ fluoro-2-(4-((2-nitro-1Himidazol-1-yl)methyl)1H-1,2,3,-triazol-1-yl)-propan-1-ol) (87) and ${ }^{18} \mathrm{~F}$-fluoromisonidazole ( $\left[{ }^{18} \mathrm{~F}\right]-\mathrm{MISO}$ ) (88) have been positively tested in imaging of hypoxia in tumors; however, they remain to be validated in atherosclerosis imaging. Recently, hypoxic atherosclerotic areas in LDLr ApoB ${ }^{100 / 100}$ mice were successfully imaged ex vivo using $\left[{ }^{18} \mathrm{~F}\right]$-EF5 and PET scan. $\left[{ }^{18} \mathrm{~F}\right]-\mathrm{EF} 5$ signal on autoradiography was significantly higher in atherosclerotic plaques compared to the normal arterial vessel wall and uptake was independent of calcification, and surprisingly inflammatory state of the plaque (11). Recent papers and the above described metabolic shift in plaque macrophages suggest that the metabolic marker ${ }^{18} \mathrm{~F}$-fluorodeoxyglucose $\left({ }^{18} \mathrm{~F}-\mathrm{FDG}\right)$ can be used as an indirect marker of hypoxia in vivo. ${ }^{18} \mathrm{~F}$-FDG is a glucose analogue PET tracer, which is taken up particularly by highly proliferative and glycolytically active cells and has hence also been linked with inflammation. In vitro data show that glucose uptake in human macrophages is stimulated to a greater extent by hypoxic conditions as compared to inflammatory triggers (65), suggesting that ${ }^{18} \mathrm{~F}-\mathrm{FDG}$ uptake can be used to visualize plaque hypoxia. In vivo, ${ }^{18} \mathrm{~F}-\mathrm{FDG}$ positivity has been linked with tumor inflammation and also atherosclerotic plaques could be detected in oncology patients (89-92). However, there is conflicting evidence for the effectiveness of ${ }^{18} \mathrm{~F}-\mathrm{FDG}$ as a hypoxic marker. Whereas ${ }^{18} \mathrm{~F}$-FDG did not correlate well with ${ }^{18} \mathrm{~F}$-MISO in sarcomas (93), ${ }^{18} \mathrm{~F}$-FDG PET signal positivity correlated with hypoxia-mediated gene expression in murine atherosclerosis, including genes like HIF-1 $\alpha$ and VEGF (94). Only few human atherosclerosis imaging studies have been performed so far (95). These correlate the ${ }^{18} \mathrm{~F}-\mathrm{FDG}$ uptake to macrophage 
presence in the plaque, and symptomatic unstable plaques show greater ${ }^{18} \mathrm{~F}$-FDG uptake compared to advanced plaques $(92,94,96,97)$. Thus, ${ }^{18} \mathrm{~F}-\mathrm{FDG}$ represents an indirect tool to measure atherosclerotic plaque hypoxia and inflammation in atherosclerotic plaques and was recently successfully used to monitor decreases in human plaque inflammation under atorvastatin, pioglitazone and the HDL raising compound dalcetrapib (98). A combination with imidazole-based hypoxia PET scan markers and ${ }^{18} \mathrm{~F}-\mathrm{FDG}$ may prove useful in diagnosis of plaque phenotypes and plaque progression.

\section{CONCLUSION}

Recent studies have confirmed the presence of hypoxia in atherosclerotic plaques, and its co-localization with macrophages. Systemic hypoxia sleep apnea models and clinical studies reveal the pro-atherosclerotic role of hypoxia and mechanistically link hypoxia with impaired macrophage function. Intracellular lipid accumulation and LDL oxidation and macrophage glucose metabolism are induced, resulting in ROS signaling, lactate production and acceleration of disease.

From this evidence we conclude that hypoxia may well represent one of the main drivers of atherosclerosis by interfering with macrophage function. Additionally, recent evidence interlinks plaque hypoxia with macrophage metabolic changes; however a causal relationship remains to be established. 


\section{FUNDING}

Research was supported by a VENI fellowship of the Netherlands Organization of Scientific research (to J.C.S. 016.116.017). 


\section{REFERENCES}

1. Crawford DW, Kramsch DM. The oxygen environment of the arterial media in early rabbit hypertension. Exp Mol Pathol. 1988 Oct;49(2):215-33.

2. Martin JF, Booth RF, Moncada S. Arterial wall hypoxia following hyperfusion through the vasa vasorum is an initial lesion in atherosclerosis. Eur J Clin Invest. 1990 Dec;20(6):588-92.

3. Heughan C, Niinikoski J, Hunt TK. Oxygen tensions in lesions of experimental atherosclerosis of rabbits. Atherosclerosis. 17(3):361-7.

4. Jurrus ER, Weiss HS. In vitro tissue oxygen tensions in the rabbit aortic arch. Atherosclerosis. 1977 Nov;28(3):223-32.

5. Niinikoski J, Heughan C, Hunt TK. Oxygen tensions in the aortic wall of normal rabbits. Atherosclerosis. 1973;17(3):353-9.

6. Bjornheden T, Evaldsson M, Wiklund O. A Method for the Assessment of Hypoxia in the Arterial Wall, With Potential Application In vivo. Arterioscler Thromb Vasc Biol. 1996 Jan 1;16(1):17885.

7. Bjornheden T, Levin M, Evaldsson M, Wiklund O. Evidence of Hypoxic Areas Within the Arterial Wall In vivo. Arterioscler Thromb Vasc Biol. 1999 Apr 1;19(4):870-6.

8. Joseph P, Jaiswal AK, Stobbe CC, Chapman JD. The role of specific reductases in the intracellular activation and binding of 2-nitroimidazoles. Int J Radiat Oncol Biol Phys. 1994 May 15;29(2):351-5.

9. NPI Inc. Hypoxyprobe [Internet]. hydroxyprobe.com. 2013. Available from: www. hypoxyprobe.com

10. Sluimer JC, Gasc J-M, van Wanroij JL, Kisters N, Groeneweg M, Sollewijn Gelpke MD, et al. Hypoxia, hypoxia-inducible transcription factor, and macrophages in human atherosclerotic plaques are correlated with intraplaque angiogenesis. J Am Coll Cardiol. 2008 Apr 1;51(13):1258-65.

11. Silvola JMU, Saraste A, Forsback S, Laine VJO, Saukko P, Heinonen SE, et al. Detection of hypoxia by [18F]EF5 in atherosclerotic plaques in mice. Arterioscler Thromb Vasc Biol. 2011 May;31(5):1011-5.

12. Ramkhelawon B, Yang Y, van Gils JM, Hewing B, Rayner KJ, Parathath S, et al. Hypoxia Induces Netrin-1 and Unc5b in Atherosclerotic Plaques: Mechanism for Macrophage Retention and Survival. Arterioscler Thromb Vasc Biol. 2013 Apr 18;33(6):1180-8.

13. Rey S, Semenza GL. Hypoxia-inducible factor-1-dependent mechanisms of vascularization and vascular remodelling. Cardiovasc Res. 2010 May 1;86(2):236-42.

14. Gao L, Chen Q, Zhou X, Fan L. The role of hypoxia-inducible factor 1 in atherosclerosis. J Clin Pathol. 2012 Oct;65(10):872-6.

15. Semenza GL. Hypoxia-inducible factor 1: regulator of mitochondrial metabolism and mediator of ischemic preconditioning. Biochim Biophys Acta. 2011 Jul;1813(7):1263-8.

16. Semenza GL. Hypoxia-inducible factors in physiology and medicine. Cell. 2012 Feb 3;148(3):399-408.

17. Wang GL, Jiang BH, Rue EA, Semenza GL. Hypoxia-inducible factor 1 is a basic-helixloop-helix-PAS heterodimer regulated by cellular $\mathrm{O}_{2}$ tension. Proc Natl Acad Sci U S A. 1995 Jun 6;92(12):5510-4.

18. Richard DE, Berra E, Pouyssegur J. Nonhypoxic pathway mediates the induction of hypoxiainducible factor 1alpha in vascular smooth muscle cells. J Biol Chem. 2000 Sep 1;275(35):26765-71.

19. Görlach A, Diebold I, Schini-Kerth VB, Berchner-Pfannschmidt U, Roth U, Brandes RP, et al. Thrombin activates the hypoxia-inducible factor-1 signaling pathway in vascular smooth muscle cells: Role of the p22(phox)-containing NADPH oxidase. Circ Res. 2001 Jul 6;89(1):47-54.

20. Nishi K, Oda T, Takabuchi S, Oda S, Fukuda K, Adachi T, et al. LPS induces hypoxiainducible factor 1 activation in macrophage-differentiated cells in a reactive oxygen species-dependent 
manner. Antioxid Redox Signal. 2008 May;10(5):983-95.

21. Shatrov VA, Sumbayev V V, Zhou J, Brüne B. Oxidized low-density lipoprotein (oxLDL) triggers hypoxia-inducible factor-1alpha (HIF-1alpha) accumulation via redox-dependent mechanisms. Blood. 2003 Jun 15;101(12):4847-9.

22. De Ponti C, Carini R, Alchera E, Nitti MP, Locati M, Albano E, et al. Adenosine A2a receptor-mediated, normoxic induction of HIF-1 through PKC and PI-3K-dependent pathways in macrophages. J Leukoc Biol. 2007 Aug;82(2):392-402.

23. The Lancet. The global burden of disease study 2010 [Internet]. The Lancet. 2012 [cited 2013 May 1]. Available from: http://www.thelancet.com/themed/global-burden-of-disease

24. Baguet J-P, Barone-Rochette G, Tamisier R, Levy P, Pépin J-L. Mechanisms of cardiac dysfunction in obstructive sleep apnea. Nat Rev Cardiol. 2012 Dec;9(12):679-88.

25. Mooe T, Franklin KA, Holmström K, Rabben T, Wiklund U. Sleep-disordered breathing and coronary artery disease: long-term prognosis. Am J Respir Crit Care Med. 2001 Nov 15;164(10 Pt 1):1910-3.

26. Drager LF, Polotsky VY, Lorenzi-Filho G. Obstructive sleep apnea: an emerging risk factor for atherosclerosis. Chest. 2011 Aug;140(2):534-42.

27. Bradley TD, Floras JS. Obstructive sleep apnoea and its cardiovascular consequences. Lancet. 2009 Jan 3;373(9657):82-93.

28. Mokhlesi B, Gozal D. Update in sleep medicine 2009. Am J Respir Crit Care Med. 2010 Mar 15;181(6):545-9.

29. Lévy P, Pépin J-L, Arnaud C, Baguet J-P, Dematteis M, Mach F. Obstructive sleep apnea and atherosclerosis. Prog Cardiovasc Dis. 2009;51(5):400-10.

30. Yaggi HK, Concato J, Kernan WN, Lichtman JH, Brass LM, Mohsenin V. Obstructive sleep apnea as a risk factor for stroke and death. N Engl J Med. 2005 Nov 10;353(19):2034-41.

31. Fletcher EC. Invited review: Physiological consequences of intermittent hypoxia: systemic blood pressure. J Appl Physiol. 2001 Apr;90(4):1600-5.

32. Yamauchi M, Kimura H. Oxidative stress in obstructive sleep apnea: putative pathways to the cardiovascular complications. Antioxid Redox Signal. 2008 Apr;10(4):755-68.

33. Jun J, Reinke C, Bedja D, Berkowitz D, Bevans-Fonti S, Li J, et al. Effect of intermittent hypoxia on atherosclerosis in apolipoprotein E-deficient mice. Atherosclerosis. 2010 Apr;209(2):381-6.

34. Savransky V, Nanayakkara A, Li J, Bevans S, Smith PL, Rodriguez A, et al. Chronic intermittent hypoxia induces atherosclerosis. Am J Respir Crit Care Med. 2007 Jun 15;175(12):1290-7.

35. Nakano D, Hayashi T, Tazawa N, Yamashita C, Inamoto S, Okuda N, et al. Chronic hypoxia accelerates the progression of atherosclerosis in apolipoprotein E-knockout mice. Hypertens Res. 2005 Oct;28(10):837-45.

36. Fang G, Song D, Ye X, Mao S, Liu G, Liu SF. Chronic intermittent hypoxia exposure induces atherosclerosis in ApoE knockout mice: role of NF- $x$ B p50. Am J Pathol. 2012 Nov;181(5):1530-9.

37. Büchner NJ, Quack I, Woznowski M, Stähle C, Wenzel U, Rump LC. Microvascular endothelial dysfunction in obstructive sleep apnea is caused by oxidative stress and improved by continuous positive airway pressure therapy. Respiration. 2011 Jan;82(5):409-17.

38. Drager LF, Bortolotto LA, Figueiredo AC, Krieger EM, Lorenzi GF. Effects of continuous positive airway pressure on early signs of atherosclerosis in obstructive sleep apnea. Am J Respir Crit Care Med. 2007 Oct 1;176(7):706-12.

39. Kudchodkar BJ, Wilson J, Lacko A, Dory L. Hyperbaric oxygen reduces the progression and accelerates the regression of atherosclerosis in rabbits. Arterioscler Thromb Vasc Biol. 2000 Jun;20(6):1637-43.

40. Kudchodkar BJ, Pierce A, Dory L. Chronic hyperbaric oxygen treatment elicits an antioxidant response and attenuates atherosclerosis in apoE knockout mice. Atherosclerosis. 2007 Jul;193(1):28-35. 
41. Kudchodkar B, Jones H, Simecka J, Dory L. Hyperbaric oxygen treatment attenuates the pro-inflammatory and immune responses in apolipoprotein E knockout mice. Clin Immunol. 2008 Sep;128(3):435-41.

42. Karadurmus N, Sahin M, Tasci C, Naharci I, Ozturk C, Ilbasmis S, et al. Potential benefits of hyperbaric oxygen therapy on atherosclerosis and glycaemic control in patients with diabetic foot. Endokrynol Pol. 2010;61(3):275-9.

43. Chen S-Y, Huang E, Wang V, Fan Y-M, Ho C-F, Yip P-K. Improvement of clinical outcome and cerebral perfusion in a patient of atherosclerotic cerebral infarction after repetitive hyperbaric oxygen treatment--a case report and literature review. Undersea Hyperb Med. 2011;38(5):375-9.

44. Unfirer S, Kibel A, Drenjancevic-Peric I. The effect of hyperbaric oxygen therapy on blood vessel function in diabetes mellitus. Med Hypotheses. 2008 Nov;71(5):776-80.

45. Lokmic Z, Musyoka J, Hewitson TD, Darby IA. Hypoxia and hypoxia signaling in tissue repair and fibrosis. Int Rev Cell Mol Biol. 2012 Jan;296:139-85.

46. Li L, Liu B, Håversen L, Lu E, Magnusson LU, Ståhlman M, et al. The importance of GLUT3 for de novo lipogenesis in hypoxia-induced lipid loading of human macrophages. PLoS One. 2012 Jan;7(8):e42360.

47. Na T-Y, Lee H-J, Oh H-J, Huh S, Lee I-K, Lee M-O. Positive cross-talk between hypoxia inducible factor- $1 \alpha$ and liver $\mathrm{X}$ receptor $\alpha$ induces formation of triglyceride-loaded foam cells. Arterioscler Thromb Vasc Biol. 2011 Dec;31(12):2949-56.

48. Asplund A, Fridén V, Stillemark-Billton P, Camejo G, Bondjers G. Macrophages exposed to hypoxia secrete proteoglycans for which LDL has higher affinity. Atherosclerosis. 2011 Mar;215(1):7781.

49. Parathath S, Yang Y, Mick S, Fisher EA. Hypoxia in murine atherosclerotic plaques and its adverse effects on macrophages. Trends Cardiovasc Med. 2013 Apr;23(3):80-4.

50. Jiang G, Li T, Qiu Y, Rui Y, Chen W, Lou Y. RNA interference for HIF-1alpha inhibits foam cells formation in vitro. Eur J Pharmacol. 2007 May 21;562(3):183-90.

51. Parathath S, Mick SL, Feig JE, Joaquin V, Grauer L, Habiel DM, et al. Hypoxia is present in murine atherosclerotic plaques and has multiple adverse effects on macrophage lipid metabolism. Circ Res. 2011 Oct 28;109(10):1141-52.

52. Song D, Fang G, Mao S-Z, Ye X, Liu G, Gong Y, et al. Chronic intermittent hypoxia induces atherosclerosis by NF- «B-dependent mechanisms. Biochim Biophys Acta. 2012 Nov;1822(11):1650-9. 53. Kanters E, Gijbels MJJ, van der Made I, Vergouwe MN, Heeringa P, Kraal G, et al. Hematopoietic NF-kappaB1 deficiency results in small atherosclerotic lesions with an inflammatory phenotype. Blood. 2004 Feb 1;103(3):934-40.

54. Drager LF, Yao Q, Hernandez KL, Shin M-K, Bevans-Fonti S, Gay J, et al. Chronic Intermittent Hypoxia Induces Atherosclerosis via Activation of Adipose Angiopoietin-like 4. Am J Respir Crit Care Med. 2013 Jan 17;

55. Li RC, Haribabu B, Mathis SP, Kim J, Gozal D. Leukotriene B4 receptor-1 mediates intermittent hypoxia-induced atherogenesis. Am J Respir Crit Care Med. 2011 Jul 1;184(1):124-31.

56. Wang B, Yan B, Song D, Ye X, Liu SF. Chronic intermittent hypoxia down-regulates endothelial nitric oxide synthase expression by an NF-xB-dependent mechanism. Sleep Med. 2013 Feb;14(2):165-71.

57. Johnson AR, Freemerman AJ, Abel E, Rathmell J, Makowski L. Glucose metabolism is linked to the inflammatory status of macrophages. BMC Proc. 2012;6(Suppl 3):P62.

58. Fang H-Y, Hughes R, Murdoch C, Coffelt SB, Biswas SK, Harris AL, et al. Hypoxia-inducible factors 1 and 2 are important transcriptional effectors in primary macrophages experiencing hypoxia. Blood. 2009 Jul 23;114(4):844-59.

59. Oda T, Hirota K, Nishi K, Takabuchi S, Oda S, Yamada H, et al. Activation of hypoxiainducible factor 1 during macrophage differentiation. Am J Physiol Cell Physiol. 2006 Jul;291(1):C104-13. 
60. Warburg O, Wind F, Negelein E. The Metabolism of Tumors in the Body. J Gen Physiol. 1927/03/07 ed. 1927;8(6):519-30.

61. Vander Heiden MG, Cantley LC, Thompson CB. Understanding the Warburg effect: the metabolic requirements of cell proliferation. Science. 2009 May 22;324(5930):1029-33.

62. Wang R, Dillon CP, Shi LZ, Milasta S, Carter R, Finkelstein D, et al. The transcription factor Myc controls metabolic reprogramming upon T lymphocyte activation. Immunity. 2011 Dec 23;35(6):871-82.

63. Murdoch C, Muthana M, Lewis CE. Hypoxia regulates macrophage functions in inflammation. J Immunol. 2005 Nov 15;175(10):6257-63.

64. Kawaguchi T, Veech RL, Uyeda K. Regulation of energy metabolism in macrophages during hypoxia. Roles of fructose 2,6-bisphosphate and ribose 1,5-bisphosphate. J Biol Chem. 2001 Jul 27;276(30):28554-61.

65. Folco EJ, Sheikine Y, Rocha VZ, Christen T, Shvartz E, Sukhova GK, et al. Hypoxia but not inflammation augments glucose uptake in human macrophages: Implications for imaging atherosclerosis with 18fluorine-labeled 2-deoxy-D-glucose positron emission tomography. J Am Coll Cardiol. 2011 Aug 2;58(6):603-14.

66. O'Neill LAJ, Hardie DG. Metabolism of inflammation limited by AMPK and pseudostarvation. Nature. 2013 Jan 17;493(7432):346-55.

67. Leppänen O, Björnheden T, Evaldsson M, Borén J, Wiklund O, Levin M. ATP depletion in macrophages in the core of advanced rabbit atherosclerotic plaques in vivo. Atherosclerosis. 2006 Oct;188(2):323-30.

68. Shantha GPS, Wasserman B, Astor BC, Coresh J, Brancati F, Sharrett AR, et al. Association of blood lactate with carotid atherosclerosis: The Atherosclerosis Risk in Communities (ARIC) Carotid MRI Study. Atherosclerosis. 2013 Feb 28;228(1):249-55.

69. Oörni K, Kovanen PT. Enhanced extracellular lipid accumulation in acidic environments. Curr Opin Lipidol. 2006 Oct;17(5):534-40.

70. Khazaei M, Nematbakhsh M. Effect of experimentally induced metabolic acidosis on aortic endothelial permeability and serum nitric oxide concentration in normal and high-cholesterol fed rabbits. Arch Med Sci. 2012 Sep 8;8(4):719-23.

71. Murphy MP. How mitochondria produce reactive oxygen species. Biochem J. 2009 Jan 1;417(1):1-13.

72. Violi F, Basili S, Nigro C, Pignatelli P. Role of NADPH oxidase in atherosclerosis. Future Cardiol. 2009 Jan;5(1):83-92.

73. Vogiatzi G, Tousoulis D, Stefanadis C. The role of oxidative stress in atherosclerosis. Hellenic J Cardiol. 2009;50(5):402-9.

74. Klimova T, Chandel NS. Mitochondrial complex III regulates hypoxic activation of HIF. Cell Death Differ. 2008 Apr;15(4):660-6.

75. Tavakoli S, Asmis R. Reactive oxygen species and thiol redox signaling in the macrophage biology of atherosclerosis. Antioxid Redox Signal. Mary Ann Liebert, Inc. 140 Huguenot Street, 3rd Floor New Rochelle, NY 10801 USA; 2012 Dec 15;17(12):1785-95.

76. Schaefer CA, Kuhlmann CRW, Weiterer S, Fehsecke A, Abdallah Y, Schaefer C, et al. Statins inhibit hypoxia-induced endothelial proliferation by preventing calcium-induced ROS formation. Atherosclerosis. 2006 Apr;185(2):290-6.

77. Christou K, Kostikas K, Pastaka C, Tanou K, Antoniadou I, Gourgoulianis KI. Nasal continuous positive airway pressure treatment reduces systemic oxidative stress in patients with severe obstructive sleep apnea syndrome. Sleep Med. 2009 Jan;10(1):87-94.

78. Del Ben M, Fabiani M, Loffredo L, Polimeni L, Carnevale R, Baratta F, et al. Oxidative stress mediated arterial dysfunction in patients with obstructive sleep apnoea and the effect of continuous positive airway pressure treatment. BMC Pulm Med. 2012 Jan;12:36. 
79. Schulz R, Mahmoudi S, Hattar K, Sibelius U, Olschewski H, Mayer K, et al. Enhanced release of superoxide from polymorphonuclear neutrophils in obstructive sleep apnea. Impact of continuous positive airway pressure therapy. Am J Respir Crit Care Med. 2000 Aug;162(2 Pt 1):566-70.

80. Lavie L. Oxidative stress inflammation and endothelial dysfunction in obstructive sleep apnea. Front Biosci (Elite Ed). 2012 Jan;4:1391-403.

81. Fujisaka S, Usui I, Ikutani M, Aminuddin A, Takikawa A, Tsuneyama K, et al. Adipose tissue hypoxia induces inflammatory M1 polarity of macrophages in an HIF-1 $\alpha$-dependent and HIF-1 $\alpha$ independent manner in obese mice. Diabetologia. 2013 Mar 15;56(6):1403-12.

82. Werno C, Menrad H, Weigert A, Dehne N, Goerdt S, Schledzewski K, et al. Knockout of HIF- $1 \alpha$ in tumor-associated macrophages enhances M2 polarization and attenuates their proangiogenic responses. Carcinogenesis. 2010 Oct;31(10):1863-72.

83. Lewis C, Murdoch C. Macrophage responses to hypoxia: implications for tumor progression and anti-cancer therapies. Am J Pathol. 2005 Sep;167(3):627-35.

84. Stöger JL, Gijbels MJJ, van der Velden S, Manca M, van der Loos CM, Biessen EAL, et al. Distribution of macrophage polarization markers in human atherosclerosis. Atherosclerosis. 2012 Dec;225(2):461-8.

85. Laoui D, Van Overmeire E, Movahedi K, Van den Bossche J, Schouppe E, Mommer C, et al. Mononuclear phagocyte heterogeneity in cancer: different subsets and activation states reaching out at the tumor site. Immunobiology. 2011 Nov;216(11):1192-202.

86. Abramson Cancer Center of the University of Pennsylvania. Fluorine F18-EF5 and Fludeoxyglucose F18 Positron Emission Tomography in Assessing Hypoxia and Glycolysis in Patients With Stage I, Stage II, or Stage III Non-Small Cell Lung Cancer - Full Text View - ClinicalTrials.gov [Internet]. clinicaltrials.gov. 2012 [cited 2013 Apr 23]. Available from: http://www.clinicaltrials.gov/ ct $2 /$ show $/$ NCT01017133?term $=$ EF5\&rank $=7$

87. van Loon J, Janssen MHM, Ollers M, Aerts HJWL, Dubois L, Hochstenbag M, et al. PET imaging of hypoxia using [18F]HX4: a phase I trial. Eur J Nucl Med Mol Imaging. 2010 Aug;37(9):16638.

88. Tachibana I, Nishimura Y, Shibata T, Kanamori S, Nakamatsu K, Koike R, et al. A prospective clinical trial of tumor hypoxia imaging with 18F-fluoromisonidazole positron emission tomography and computed tomography (F-MISO PET/CT) before and during radiation therapy. J Radiat Res. 2013 Apr 14;

89. Dunphy MPS, Freiman A, Larson SM, Strauss HW. Association of vascular 18F-FDG uptake with vascular calcification. J Nucl Med. 2005 Aug;46(8):1278-84.

90. Tatsumi M, Cohade C, Nakamoto Y, Wahl RL. Fluorodeoxyglucose uptake in the aortic wall at PET/CT: possible finding for active atherosclerosis. Radiology. 2003 Dec;229(3):831-7.

91. Yun M, Yeh D, Araujo LI, Jang S, Newberg A, Alavi A. F-18 FDG uptake in the large arteries: a new observation. Clin Nucl Med. 2001 Apr;26(4):314-9.

92. Bural GG, Torigian DA, Chamroonrat W, Houseni M, Chen W, Basu S, et al. FDG-PET is an effective imaging modality to detect and quantify age-related atherosclerosis in large arteries. Eur J Nucl Med Mol Imaging. 2008 Mar;35(3):562-9.

93. Rajendran JG, Wilson DC, Conrad EU, Peterson LM, Bruckner JD, Rasey JS, et al. [(18)F] FMISO and [(18)F]FDG PET imaging in soft tissue sarcomas: correlation of hypoxia, metabolism and VEGF expression. Eur J Nucl Med Mol Imaging. 2003 May;30(5):695-704.

94. Hag AMF, Pedersen SF, Christoffersen C, Binderup T, Jensen MM, Jørgensen JT, et al. (18) F-FDG PET imaging of murine atherosclerosis: association with gene expression of key molecular markers. PLoS One. 2012 Jan; 7(11):e50908.

95. Fayad ZA, Mani V, Woodward M, Kallend D, Abt M, Burgess T, et al. Safety and efficacy of dalcetrapib on atherosclerotic disease using novel non-invasive multimodality imaging (dal-PLAQUE): a randomised clinical trial. Lancet. 2011 Oct 29;378(9802):1547-59. 
96. Tawakol A, Migrino RQ, Bashian GG, Bedri S, Vermylen D, Cury RC, et al. In vivo 18F-fluorodeoxyglucose positron emission tomography imaging provides a noninvasive measure of carotid plaque inflammation in patients. J Am Coll Cardiol. 2006 Nov 7;48(9):1818-24.

97. Kwee RM, Truijman MTB, Mess WH, Teule GJJ, ter Berg JWM, Franke CL, et al. Potential of integrated $[18 \mathrm{~F}]$ fluorodeoxyglucose positron-emission tomography/CT in identifying vulnerable carotid plaques. AJNR Am J Neuroradiol. 2011 May;32(5):950-4.

98. Rosenbaum D, Millon A, Fayad ZA. Molecular imaging in atherosclerosis: FDG PET. Curr Atheroscler Rep. 2012 Oct;14(5):429-37. 



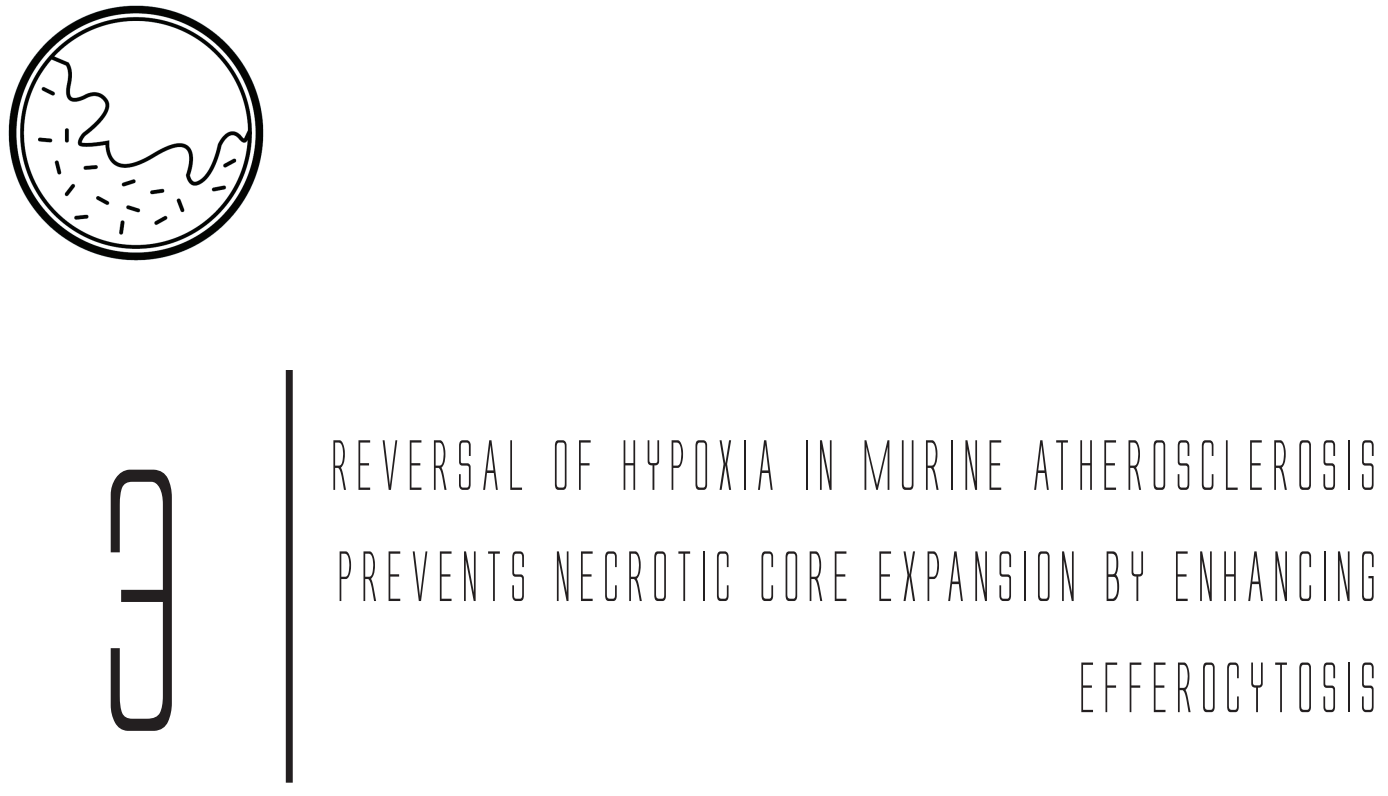

margch e', theelen tl’, demandt Jaf, Jeuriggen m, van gink m, Verjang R, jangsen A, CLEUTJENS JP, MEEX SJ, DONNERS MM, HAENEN GR, SCHALKWIJK CG, DUBOIS LJ, LAMBIN P,

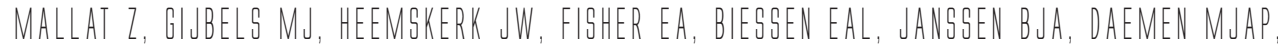
SLUIMER JC 'Authors contributed equally arterlogCler thromb VagC BIOL. 2014 DeC;94(I2):2545-59. 



\section{ABSTRACT}

\section{BACKGROUND}

Advanced murine and human plaques are hypoxic, but it remains unclear whether plaque hypoxia is causally related to atherogenesis. Here, we tested the hypothesis that reversal of hypoxia in atherosclerotic plaques by breathing hyperoxic carbogen gas will prevent atherosclerosis.

\section{APPROACH AND RESULTS}

$\mathrm{LDLr}^{-/}$mice were fed a western-type diet, exposed to carbogen $\left(95 \% \mathrm{O}_{2}, 5 \% \mathrm{CO}_{2}\right)$ or air and the effect on plaque hypoxia, size and phenotype was studied.

First, the hypoxic marker pimonidazole was detected in murine $\mathrm{LDLr}^{-/}$plaque macrophages from plaque initiation onwards. Second, the efficacy of breathing carbogen (90min, single exposure) was studied. Compared to air, carbogen increased arterial blood $\mathrm{pO}_{2} 5$-fold in $\mathrm{LDLr}^{-/}$mice, and reduced plaque hypoxia in advanced plaques of the aortic root $(-32 \%)$ and arch $(-84 \%)$. Finally, the effect of repeated carbogen exposure on progression of atherosclerosis was studied in $\mathrm{LDLr}^{-/}$mice fed a high cholesterol diet for an initial 4 weeks, followed by 4 weeks of diet and carbogen or air (both 90min/ day). Carbogen reduced plaque hypoxia (-40\%), necrotic core size $(-37 \%)$, and TUNEL $^{+}$ apoptotic cell content $(-50 \%)$ and increased efferocytosis of apoptotic cells by MAC3 ${ }^{+}$ macrophages $(+36 \%)$ in advanced plaques of the aortic root. Plaque size, plasma cholesterol, hematopoiesis and systemic inflammation were unchanged. In vitro, hypoxia hampered efferocytosis by bone marrow-derived macrophages, which was dependent on the receptor MerTK.

\section{CONCLUSION}

Carbogen restored murine plaque oxygenation and prevented necrotic core expansion by enhancing efferocytosis, likely via MerTK. Thus, plaque hypoxia is causally related to necrotic core expansion. 


\section{INTRODUCTION}

In many pathophysiological conditions, reduced oxygen tension (hypoxia) is a known stimulus of inflammation, angiogenesis and apoptosis $(1,2)$. As the progression of atherosclerosis is driven by the very same processes (3-5), we recently investigated if hypoxia was present in atherosclerosis. Indeed, plaque hypoxia was detected specifically in macrophages of advanced human atherosclerotic lesions using administration of the hypoxia-specific marker pimonidazole (6), as well as in advanced rabbit and murine plaques (7-10). However, whether plaque hypoxia is an innocent bystander-effect of macrophage influx, or a driving force behind plaque progression is still unknown.

As the in vitro effects of hypoxia on macrophage function are pro-angiogenic, proinflammatory (11) and anti-fibrotic (12), we hypothesized that hypoxia in plaque macrophages is pro-atherogenic. Thus, reoxygenation would be expected to prevent plaque progression. An attractive strategy to achieve plaque reoxygenation may be the breathing of hyperoxic gas carbogen, composed of $95 \% \mathrm{O}_{2}$ and $5 \% \mathrm{CO}_{2}$, as this successfully alleviated tumor hypoxia (13-15). Hyperoxic carbogen will increase oxygen dissolved in blood, thus $\mathrm{pO}_{2}$, rather than enhancing already maximal hemoglobin saturation. Additionally, the $\mathrm{CO}_{2}$ component of carbogen contributes to increased oxygen supply: $5 \% \mathrm{CO}_{2}$ ensures a respiratory drive, prevents pulmonary vasodilation associated with $100 \% \mathrm{O}_{2}$ and shifts the hemoglobin- $\mathrm{O}_{2}$ dissociation curve towards facilitated oxygen delivery (16). Thus, oxygen blood content, oxygen delivery, and oxygen diffusion are vastly improved, and enhance tissue oxygenation. Therefore, we hypothesized that carbogen delivery will reverse hypoxia in murine atherosclerotic plaques, and thus reverse hypoxia-associated effects on plaque inflammation and progression. 


\section{MATERIAL AND METHODS}

\section{ANIMALS}

Mouse experiments were approved by the regulatory authority of the Maastricht University Medical Center, and were performed in compliance with the Dutch government guidelines. All mice were bred at least 9x on C57/B16 background. Male $\mathrm{LDLr}^{-/}$were obtained from an in-house breeding colony, originally derived from Charles River (Wilmington, MA, USA). Mer tyrosine kinase domain deficient $\left(\right.$ MerTK $\left.^{\mathrm{kd}}\right)(17,18)$ and $\mathrm{CD} 36$ deficient mice $\left(\mathrm{CD} 36^{-/}\right)(19)$ and wildtype control mice were previously described.

\section{BLOOD GAS ANALYSIS}

An arterial catheter (20) was implanted in five $\mathrm{LDLr}^{-/}$mice on chow using isoflurane anesthesia (1.5-2\%) and peri-operative ibuprofen (s.c). The right femoral artery was exposed, and a heat-stretched polyethylene- 25 cannula was inserted $(1.5 \mathrm{~cm})$ and subcutaneously guided to the neck of the mouse. Here the catheter was fixed, extended, filled with heparinized saline $(10 \mathrm{U} / \mathrm{ml})$, and plugged. The mice were allowed to recover $24 \mathrm{hrs}$ before measurements were made. Mice were placed in induction chambers and the arterial line was extended through the outlet of the chamber to allow blood sampling without altering the continuous carbogen flow (5L/min, normobaric, NTG Sol). Arterial blood $(70 \mu \mathrm{l})$ was collected before and after 30 minutes of flow, and 5 minutes after flow cessation and analyzed within 5 minutes on a Chiron blood gas analyzer (Siemens).

\section{ATHEROSCLEROSIS AND CARBOGEN EXPOSURE}

First, hypoxia development in atherogenesis was investigated in $\mathrm{LDLr}^{-/}$mice $(\mathrm{n}=5 /$ group) fed a $0.25 \%$ cholesterol diet (special diet services, \#824171, 15\% cocoa butter, $10 \%$ maize starch, $20 \%$ casein, 40.5\% sucrose, 5.95\% cellulose) for 0, 4, 8, 12 or 16 weeks. Secondly, acute reversal of plaque hypoxia was investigated in $\mathrm{LDLr}^{-1}$ mice with advanced, hypoxic plaques ( $\mathrm{n}=5$ /group, 12 weeks of diet), which were subjected to a single 90 minute exposure of carbogen or air $\left(21 \% \mathrm{O}_{2}\right)$.

Halfway during the exposure, mice were injected intraperitoneally (IP) with the hypoxiaspecific marker pimonidazole $(100 \mathrm{mg} / \mathrm{kg}$, hypoxyprobe Omni kit, Hypoxyprobe Inc. Burlington, MA, USA) and sacrificed directly after cessation of carbogen flow. Thirdly, the effect of chronic carbogen exposure on atherogenesis was studied in thirty $\mathrm{LDLr}^{-/}$ ( $n=15$ /group), fed a $0.25 \%$ cholesterol diet for four weeks. During the four additional 
weeks of diet, mice were exposed daily to 90 minutes of carbogen or air. Twenty-four hours after the last exposure, mice were injected with pimonidazole one hour prior to sacrifice.

\section{atheroscLerotic LESION QUANTIFICATION AND IMMUNOHISTOChEMISTRY}

Mice were euthanized using a pentobarbital overdose $(100 \mathrm{mg} / \mathrm{kg})$ and blood was collected via the left ventricle for flow cytometry, absolute white blood cell counts (Coulter counter AC.T diff) and total plasma cholesterol analysis. Aortic roots, arches and organs were excised and fixed in 1\% paraformaldehyde. In additional LDLr ${ }^{-1}$ mice, white blood cells were collected from the abdominal aorta and the saphenous vein, and fixed (1\% paraformaldehyde, 2 hours) following erythrocyte lysis in iso-osmotic ammonium chloride solution. Remaining white blood cells were mixed with agar and paraffin-embedded.

Aortic roots and arches were serially sectioned, and stained with hematoxylin and eosin (HE, Sigma) for blinded quantification of the lesion areas using computerized morphometry (Leica QWin V3, Cambridge, UK). Total plaque area and lipid core content were obtained by averaging measurements of five representative (HE) sections (25 $\mu \mathrm{m}$ apart) of the aortic root and aortic arch. In the aortic arch, plaque size was quantified as the sum of the inner and outer curvature plaques, as well as all three major branches. Necrotic core was defined as a-cellular and a-nuclear plaque area containing cholesterol clefts. Atherosclerotic plaque size and necrotic core area in the aortic arch was determined in 5 representative HE stainings.Hypoxia was detected in atherosclerotic plaques (aortic root, aortic arch), murine kidney (positive control), liver, spleen, lymph nodes, thymus and white blood cells. In vivo, pimonidazole is irreversibly metabolized in living, hypoxic cells $\left(\mathrm{pO}_{2} \leq 10 \mathrm{mmHg} \sim 1 \% \mathrm{O}_{2}\right)(21)$, and metabolites form adducts onto thiol-containing proteins. These epitopes were ex vivo detected by immunohistochemistry with a rabbit polyclonal antibody (clone 2627) that only recognizes hypoxic metabolites derivatives of pimonidazole (22).

Hypoxia was quantified in images of entire organs or plaques ( $\%$ pimonidazole area/ total tissue area). Atherosclerotic plaques were further characterized for macrophage size and content $\left(\mathrm{MAC3}^{+}\right.$cells/total cells and $\mathrm{MAC3}^{+}$cells/plaque area, BD), T-cells $\left(\mathrm{CD}^{+}\right.$cells/plaque area, Miltenyi), collagen (picosirius red area/plaque area, analyzed with polarized light microscopy), proliferating cells $\left(\mathrm{Ki} 67^{+}\right.$cells/plaque area, Abcam), tissue factor ( $\mathrm{TF}$ ) ( $\mathrm{TF}^{+}$cells/plaque area, Santa Cruz), M1 inducible nitric oxide synthase (iNOS ${ }^{+}$cells/plaque area, Abcam), M2 mannose receptor $\left(\mathrm{MR}^{+}\right.$cells/adventitia, Abcam) expression and 8-hydroxy-2'deoxy-guanosine (8OH-dG, Bioconnect) for 
oxidative stress damage $\left(8 \mathrm{OH}-\mathrm{dG}^{+}\right.$cells/plaque area, Japan institute for the control of aging). Apoptosis (TUNEL ${ }^{+}$nuclei/plaque area, Roche) and clearance of apoptotic cells (efferocytosis) in situ were analyzed in entire tissue sections using TUNEL/MAC3 double immunohistochemistry as the ratio of bound/free apoptotic cells according to established methods $(23,24)$.

\section{MULTISPECTRAL IMAGE ANALYSIS}

Multispectral imaging was used to quantitatively measure colocalization of individual immunohistochemical staining products in tissue stained with multiple antibodies. Sections stained with pimonidazole and MAC3 were analyzed with the Nuance spectral imaging system (Perkin Elmer/Caliper Life Sciences, Hopkinton, MA, USA). Spectral imaging data sets were taken at 420-720 nm at $20 \mathrm{~nm}$ intervals using a DM-5000 Leica microscope system at 20x (plan apo). Spectral libraries of single-red (Vector Red), single-blue (Vector Blue) were obtained from the control slides. The resulting library was applied to the double stained slides to spectrally segregate them into individual component images using the Nuance ${ }^{\text {TM }} 3.0$ software. Pseudo-colors were assigned to unmixed images, and composite images showing co-localization were generated with the Nuance 3.0 software. Quantitative assessment of co-localization and pixel-based measurement of the individual markers per microscopic field was done with the same software.

\section{total plasma cholegterol, triglyceride, antioxidaNt capacity aNd CytOKINES}

Plasma was separated by centrifugation, and stored at $-80^{\circ} \mathrm{C}$ until further use. Standard enzymatic techniques were used for the assessment of plasma cholesterol (product no. 07-3663-5, Roche, Almere, the Netherlands) and plasma triglycerides (FS5' Ecoline REF 157609990 314; DiaSys - Diagnostic Systems GmbH, Holzheim, Germany) automated on the Cobas Fara centrifugal analyzer (Roche).

Anti-oxidant capacity of plasma was determined using the trolox equivalent antioxidant capacity (TEAC) assay, as described previously (25). In short, plasma was deproteinized with a final concentration of 5\% TCA and incubated with an ABTS radical solution (2,2'-azino-bis(3-ethylbenzthiazoline-6-sulfonic acid) diammonium salt) for 5 min. Subsequently, the reduction in absorbance at $734 \mathrm{~nm}$ was quantified. This was normalized to trolox control, a water-soluble vitamin $\mathrm{E}$ analogue. The assay reflects the amount of scavenged radicals expressed as $\mu \mathrm{M}$ trolox equivalents. 
Additionally, murine Interleukins (IL) 2, 4, 5, 6, 10, 12p70 and $1 \beta$, Interferon $\gamma($ IFN- $\gamma$ ), chemokine (C-X-C motif) ligand 1 (CXCL1, KC) and Tumor Necrosis Factor $\alpha$ $(\mathrm{TNF}-\alpha)$ were measured in plasma using a multispot sandwich immunoassay (V-plex pro-inflammatory panel 1, Meso Scale Discovery, Gaithersburg, USA), according to manufacturer's instruction.

\section{FLOW CYTOMETRY ANALYSIS}

Flow cytometry analysis was performed on cells isolated from blood, spleen, lymph nodes, and bone marrow ( $\mathrm{n}=10 /$ group). Spleens and lymph nodes were dissociated into single-cell suspensions, and enzymatically digested for dendritic cell separation using liberase and DNAse (both $0.2 \mathrm{mg} / \mathrm{ml}$, Roche) for 30 minutes in RPMI medium. Blood and spleen were subjected to erythrocyte lysis. Staining was performed using combinations of specific antibodies to detect bone marrow stem cells (lin/Sca-1/ckit; eBioscience, Bd), common myeloid progenitors (CD16/32 int, CD 34 $4^{\text {int; }}$ eBioscience, BD respectively), granulocyte-macrophage progenitors (CD16/32 high,$\left.C D 34^{\text {high }}\right)$, erythrocyte-megakaryocyte progenitors (CD16/32, CD34), monocytes (CD11b ${ }^{\text {high }}$, Ly6G- Ly6C ${ }^{\text {high/int/low; }}$ BD, eBioscience, Miltenyi respectively), granulocytes (CD11 $\mathrm{b}^{\text {high }}$, Ly6G $\left.{ }^{\text {high }}\right)$, T cells (CD3 $\varepsilon^{+}$; Miltenyi), T helper cells $\left(\mathrm{CD} 4^{+} ; \mathrm{BD}\right)$, cytotoxic T cells $\left(\mathrm{CD} 8 \mathrm{a}^{+}\right.$; $\mathrm{BD})$, effector/memory T cells (CD44 $4^{\text {high }}, \mathrm{CD} 62^{\text {low }}$; BD, eBioscience respectively), naïve T cells (CD44 $\left.{ }^{\text {low }}, \mathrm{CD} 62^{\text {high }}\right)$, regulatory T cells $\left(\mathrm{CD}^{+}, \mathrm{CD} 25^{+}, \mathrm{FoxP}^{+}\right.$; eBioscience), B cells $\left(\mathrm{B} 220^{+}\right.$; BD), cDC (CD11 $\mathrm{c}^{\text {high }}$, eBioscience), pDC (CD11c $\left.{ }^{\text {mid }}, \mathrm{B} 220^{+}, \mathrm{Ly}_{6} \mathrm{C}^{+}\right)$, and NK cells (NK1.1 ${ }^{+}$; eBioscience). Data were acquired and analyzed using a FACSCanto II and FACSdiva software (BD Bioscience).

\section{CELL CULTURE}

Bone marrow was isolated and cells were cultured for 7 days in RPMI-1640 (Gibco with Glutamax, 2g/L glucose) supplemented with 10\% FCS, 100U/ml PenicillinStreptomycin, and 15\% L929-conditioned medium to generate bone marrow-derived macrophages (BMDM). Jurkat T cells and J774 murine macrophages were cultured in RPMI-1640 supplemented with 10\% FCS, 100U/ml Penicillin Streptomycin.

Human THP-1 cells were obtained from the American Type Culture Collection (ATCC 10801, Manassas, VA). Cells were grown in RPMI 1640 medium (Gibco-Invitrogen, Grand Island, NY, USA) containing 10\% FetalClone® III (Hyclone, Pe-bio, Hogan, UT, USA) (v/v), 1\% GlutaMAXTM I (w/v) (Gibco-Invitrogen, Grand Island, NY, USA), 125 $\mathrm{mM}$ Hepes, penicillin (100 units $/ \mathrm{ml})$, and streptomycin $(100 \mu \mathrm{g} / \mathrm{ml})(\mathrm{Gibco}-$ Invitrogen, 
Grand Island, NY, USA) at $37^{\circ} \mathrm{C}$ in a humidified incubator with a $5 \% \mathrm{CO}_{2}$ atmosphere. To differentiate THP-1 cells into macrophages, cells were seeded at a density of $1 \times 10^{6}$ cells $/ \mathrm{ml}$ and incubated for 72 hours with $0.2 \mu \mathrm{M}$ phorbol 12-myristate 13-acetate (PMA, Sigma, Saint Louis, MO, USA).

Jurkat T cells were labeled with calcein-AM $(1 \mu \mathrm{g} / \mathrm{ml}$ Invitrogen $)$ prior to induction of apoptosis by UV irradiation (15 min 254nm, UVS-26, 6W bulb 0.02J/s/cm2) and added 3:1 to J774 or BMDM. Macrophages were exposed to $21 \%$ or $0.2 \% \mathrm{O}_{2}$ (Invivo2 1000 , Ruskinn technology LTD, Pencoed, UK) during 45 min of efferocytosis. After thorough washing, macrophages were dissociated and analyzed using FACS for the percentage of calcein/jurkat ${ }^{+}$macrophages. Twenty-four hours prior to efferocytosis, BMDM were

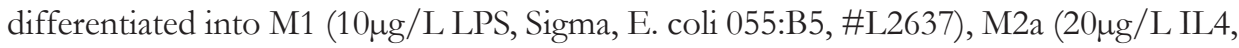

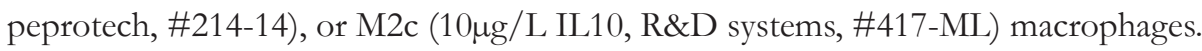

To analyze apoptosis, BMDM or J774 were exposed to hypoxia $\left(0.2 \% \mathrm{O}_{2}\right)$ for $24 \mathrm{hrs}$ or UV-irradiated for $15 \mathrm{~min}$ and apoptotic cells detected using annexin A5-Alexa594 (Invitrogen) on microscopic images. Additionally, BMDMs were cultured in normoxia or hypoxia with 7 ketocholesterol $(25 \mu \mathrm{M}$, Sigma), tunicamycin $(2.5 \mu \mathrm{g} / \mathrm{ml}$, Sigma) or oxLDL $(25 \mu \mathrm{g} / \mathrm{ml})$ supplementation for $24 \mathrm{hrs}$ and apoptotic cells were detected using TUNEL (Roche). Pimonidazole specificity was confirmed in bone marrow derived macrophages treated with $25 \mu \mathrm{g} / \mathrm{ml}$ oxLDL, LDL or control for $24 \mathrm{~h}$. Foam cell formation was confirmed by Oil-Red-O staining. Subsequently, cells were supplemented with pimonidazole $\left(100 \mu \mathrm{M}\right.$, ) and exposed to $0,0.2,1,5$ or $20 \% \mathrm{O}_{2}$ for $0 \mathrm{~h}, 1 \mathrm{~h}, 4 \mathrm{~h}$ or $16 \mathrm{~h}$. Cells were subsequently fixed, stained with anti-pimonidazole-FITC and geomean fluorescence was acquired and analyzed using a FACSCanto II and FACSdiva software (BD Bioscience). ROS production by BMDMs cultured in $21 \%$ or $0.2 \%$ oxygen for 24hours was measured using the cell permeant reagent $2^{\prime}, 7^{\prime}$-dichlorofluorescin diacetate (DCFDA, 10 $\mu \mathrm{M}$, Invitrogen, 488). Percent DCFDA positive cells were assessed via flow cytometry and $\mathrm{H}_{2} \mathrm{O}_{2}$ treated BMDMs in normoxia $(100 \mu \mathrm{M}, 10 \mathrm{~min})$ served as positive control.

\section{QUANTITATIVE RT-PCR (QPCR)}

In vitro experiments for gene expression analysis were performed in quadruplicate, and repeated twice. Total RNA was isolated and transcribed as described (26). QPCR analyses were performed from 10 ng cDNA using SYBR green and gene specific primer sets (supplemental table 1). Two housekeeping genes (cyclophilin, 18S rRNA) insensitive to changes in $\mathrm{O}_{2}$ were used to correct for differences in mRNA levels between samples. 
STATISTICAL ANALYSIS

All data are presented as mean \pm SEM. Blood gas parameters were analyzed using pairedsample testing, while independent-sample tests were done for all other parameters. Following a Shapiro-Wilk normality test, two groups were compared with student's t-test or Mann-Whitney rank-sum test. Parameters with more than two groups were analyzed using Kruskal-Wallis rank-sum test, followed by Dunn's post-hoc testing (GraphPad Prism4). Correlations between MAC3, plaque area and necrotic core with hypoxia (pimonidazole) were analyzed using univariate and multivariate linear regression (IBM SPSS Statistics 20). 


\section{RESULTS}

\section{MURINE PLAQUE MACROPhages aRE hypOXIC, INDEPENDENT OF PLAQUE gTage}

Low density lipoprotein receptor $\left(\mathrm{LDLr}^{-/}\right)$mice were fed a high cholesterol diet (HCD) for 0-16 weeks to monitor the onset and distribution of plaque hypoxia with pimonidazole at different plaque stages in the aortic root (Figure 1A).

First, specificity of pimonidazole and its detection antibody was confirmed in mice injected with vehicle or pimonidazole (Supplemental Figure 1A). Plaque hypoxia colocalized mainly with $\mathrm{MAC}^{+}$macrophages and foam cells shown by multispectral analysis of double immunohistochemistry (Figure 1C-D). Pimonidazole detection in cultured bone marrow derived macrophages (BMDM) sharply increased below 1\% $\mathrm{O}_{2}(\sim 10 \mathrm{mmHg})$ (Supplemental Figure $\left.1 \mathrm{~B}-\mathrm{C}\right)$, confirming previous data (21). Also, macrophage lipid loading per se did not result in pimonidazole positivity (Supplemental Figure 1 D). Earlier studies have also shown that oxidative stress exposure does not affect pimonidazole detection (6), overall suggesting that pimonidazole staining truly marks hypoxic macrophages.

Figure 1

A

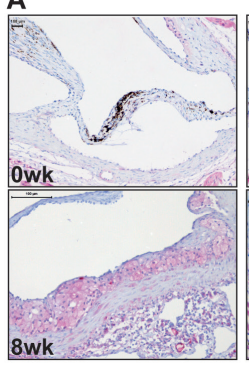

C

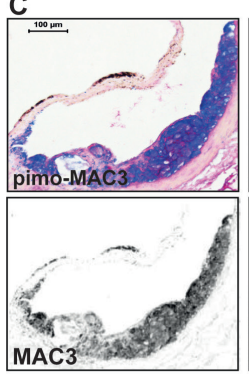

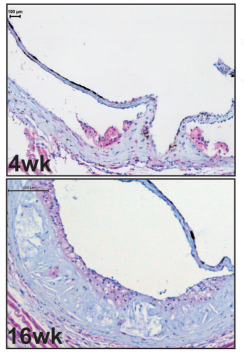

B

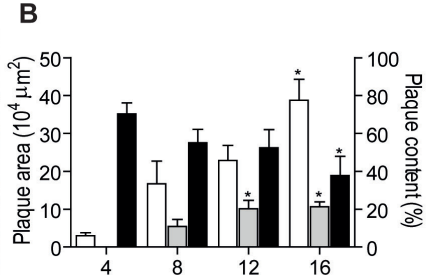

weeks of WTD

믈 Pecrue area

D

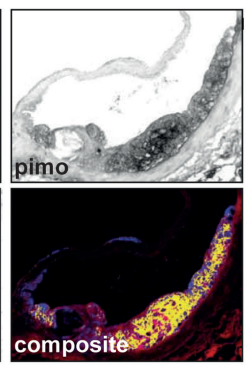

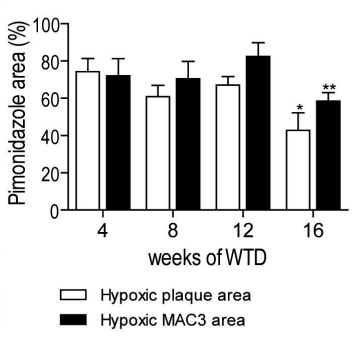

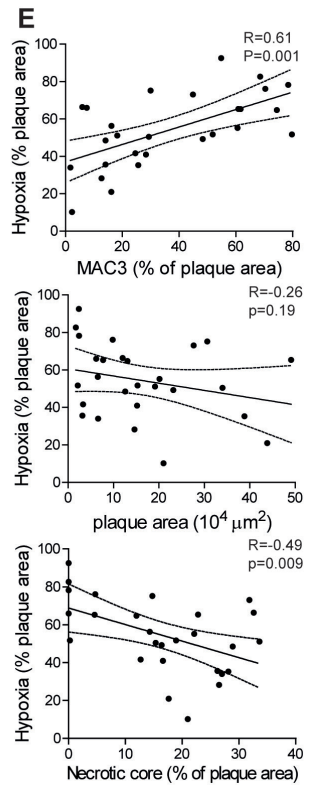

Figure 1: Murine plaque macrophages are hypoxic, independent of plaque stage

A. Representative pictures of pimonidazole-stained aortic root (red) from LDLr/- mice after 0, 4, 8 and 16 weeks of $H C D$ ( $n=5 /$ group) and B. quantification of plaque area (left y-axis), necrotic core (righty-axis) and macrophage content (righty-axis), ${ }^{*}$-value $<0.05$ vs. 4 weeks. C. Illustration of principle multispectral analysis in aortic roots from 


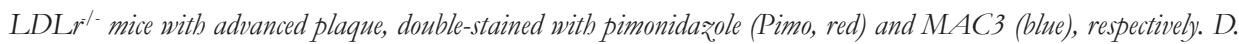
Quantification of plaque hypoxia and hypoxic macrophages (MAC3, pimonidazole double positive) in LDL $r^{1-}$ aortic root ( $n=5 /$ time point). ${ }^{*}$-value $<0.054$ vs. 16 weeks, **p-value $<0.0112$ vs. 16 weeks. (E) Scatter plots of plaque bypoxia with MAC3 (top panel), plaque area (middle panel), or necrotic core area (lower panel) with univariate linear regression line and 95\% confidence interval (dashed lines). Data from LDL $r^{\prime-}$ mice fed HCD for 4, 8, 12 and 16 weeks $(n=5$ per time point $+n=15$ air breathing controls. $n=35$ in total).

Plaque hypoxia may either arise from reduced oxygen supply to the growing plaque or from increased oxygen demand by inflammatory cells. A strong correlation between MAC3 content and pimonidazole positivity supports the latter explanation, while plaque size was not a determinant for plaque hypoxia (Figure 1E). A slight reduction in macrophage content after 16 weeks was mirrored by a reduction in hypoxia (Figure $1 \mathrm{~A}, \mathrm{D})$. Thus, murine plaque hypoxia is dictated by macrophage content, not by plaque thickness and oxygen supply. Indeed, pimonidazole was detected directly adjacent to the luminal arterial oxygen supply, in small fatty streaks after 4 weeks HCD, while being absent in the media of diseased and non-diseased arteries (Figure 1A). Although pimonidazole is metabolized by living cells only, reflected by a significant univariate correlation of plaque necrosis and hypoxia, necrosis was not a significant predictor in multivariate regression (figure $1 \mathrm{~A}, \mathrm{E}$ ). In addition, tissue-resident macrophages in inflammation-rich fatty livers and lymphoid organs of $\mathrm{HCD}$-fed $\mathrm{LDLr}^{-1}$ mice were hypoxic (Supplemental Figure $2 \mathrm{~A}-\mathrm{C}$ ). As even venous $\mathrm{pO}_{2}$ is 4-fold higher than the 10 $\mathrm{mmHg}$ detection threshold of pimonidazole (21), arterial and venous white blood cells were negative for pimonidazole (Supplemental Figure 2 D), as expected.

In conclusion, tissue resident and plaque macrophages are hypoxic. Plaque macrophages remain the only predictor of plaque hypoxia after multivariate regression, not plaque size or necrotic core content. 


\section{carbogen exposure InCreaged arterlal po, and alleviated pladue hypoxla}

Carbogen significantly increased arterial $\mathrm{pO}_{2}$ in awake $\mathrm{LDLr}^{-/}$mice (Figure 2A). Simultaneously, blood lactate was drastically reduced, while $\mathrm{pCO}_{2}$ was slightly elevated (Figure 2A), and $\mathrm{pH}$, glucose and electrolytes (data not shown) unchanged. Within minutes after cessation of carbogen breathing, all blood levels, except for lactate, returned to baseline (data not shown).

As carbogen successfully increased arterial $\mathrm{pO}_{2}$, its effect on plaque hypoxia was studied. Single 90 minute carbogen exposure in $\mathrm{LDLr}^{-/}$mice with advanced, hypoxic plaques led to a dramatic reduction of plaque hypoxia in the aortic arch and root compared to similarly sized plaques in mice breathing air (Figure 2B, C). Macrophage content, the main predictor of plaque hypoxia, was similar in both groups (Figure 2B, C). Likewise, repeated carbogen exposure was able to alleviate plaque hypoxia in $\mathrm{LDLr}^{-/}$mice, which were fed a HCD for an initial 4 weeks followed by 4 weeks of diet and carbogen or air exposure (both $90 \mathrm{~min} /$ day). Hypoxia decreased with $50 \%$ in the aortic arch and $42 \%$ in the root compared to control (Figure 2D,E), while macrophage content was not affected by carbogen gas (Figure 2D-F). Moreover, carbogen was able to significantly reoxygenate liver and spleen (Supplemental Figure 2 A-C).

To prevent oxygen toxicity possibly associated with long-term carbogen exposure, carbogen exposure was limited to 4 weeks. Chronic carbogen exposure did not affect plasma cholesterol, triglycerides, hemoglobin, or hematocrit, nor erythropoiesis, or myelopoiesis as shown by flow cytometry analysis (27) of stem cells and myeloid progenitors in bone marrow. Also it did not impact monocyte, granulocyte, T cell, B cell, and dendritic cell numbers in blood, spleen and lymph node (Supplemental Figure 3). 
Figure 2
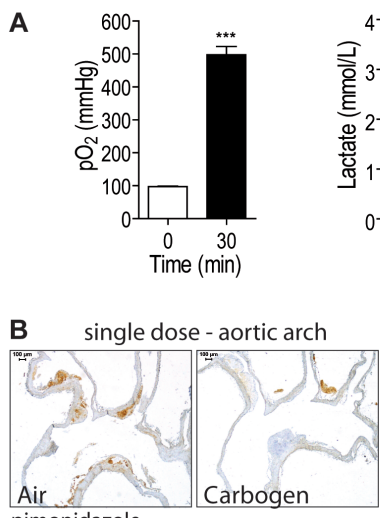

pimonidazole

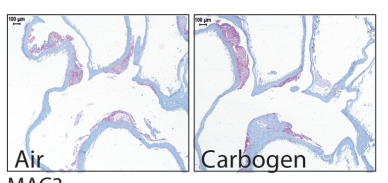

MAC3

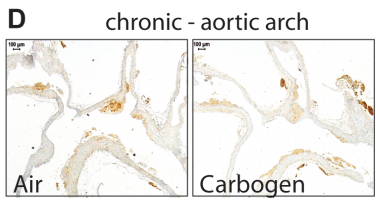

pimonidazole

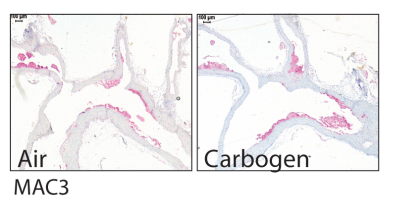

$\mathbf{F}$
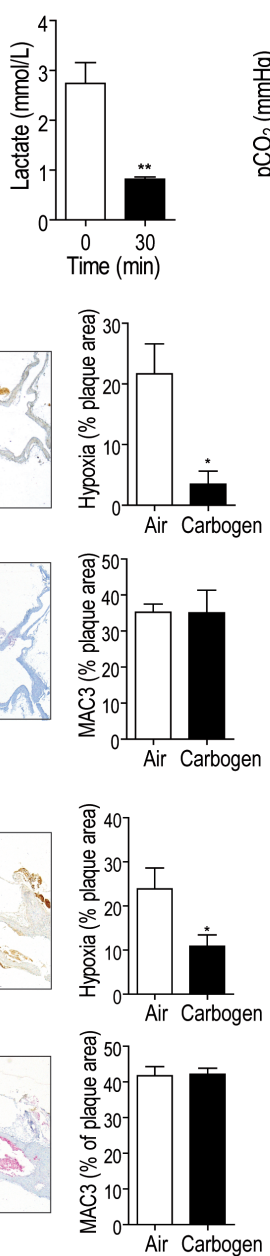
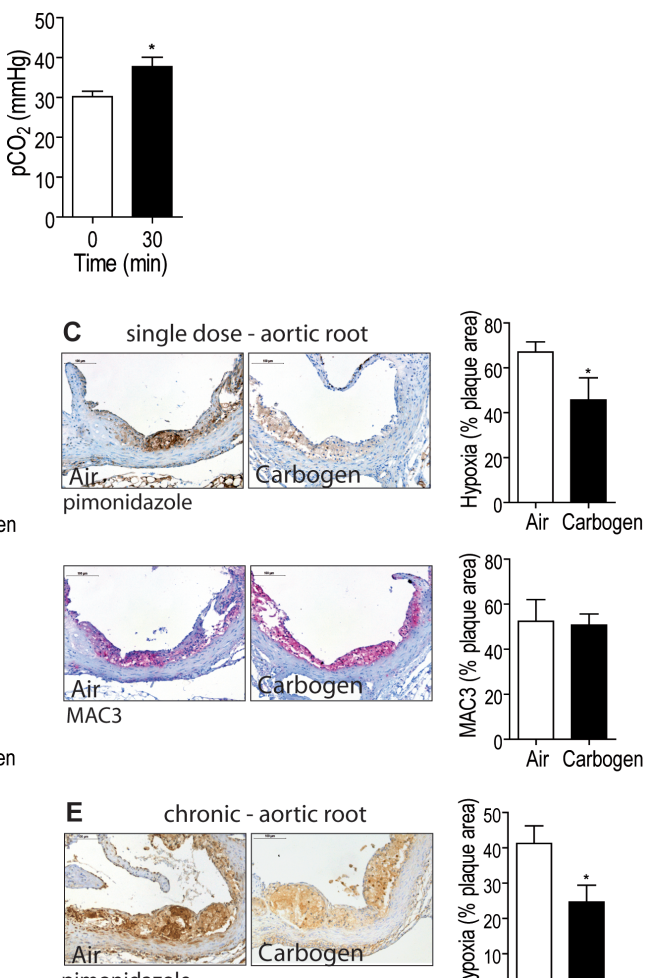

pimonidazole
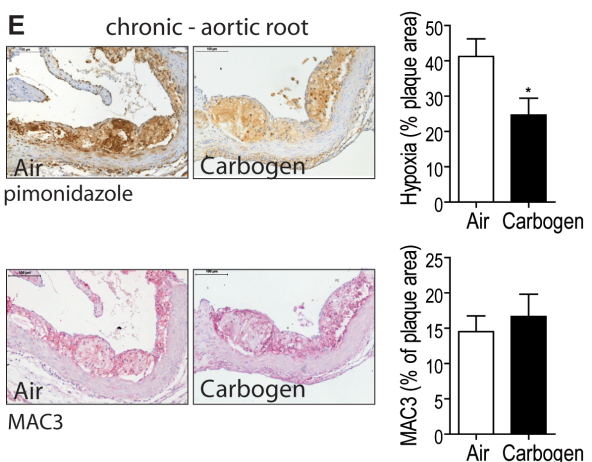

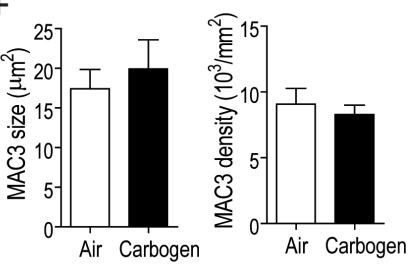

Figure 2: Carbogen increased arterial $\mathrm{pO}_{2}$ and alleviated plaque hypoxia

A. Arterial $\mathrm{OO}_{2}$ (left panel), lactate concentration (middle panel) and $p \mathrm{CO}_{2}$ (right panel) before and after 30 minutes of carbogen exposure in LDLr/- mice on chow, ${ }^{* * *}$-value $<0.001$, ${ }^{* *} p$-value $<0.01$ vs. before exposure. B. Representative pictures and quantification of pimonidazole (brown) and MAC3 (red) immunobistochemistry in aortic arch and branches of $\mathrm{LDLr} \mathrm{r}^{-}$- mice exposed to single dose of air or carbogen. C. Representative pictures and quantification of plaque hypoxia and macrophages in the aortic root upon single dose of air or carbogen. D. Representative pictures and 
quantification of plaque hypoxia and macrophages in the aortic arch and E. root of $L D L r^{1-}$ mice after chronic carbogen or air treatment. ${ }^{*}$-value $<0.05$ vs. air. F. Quantification of macrophage size (left graph) and density (right graph) upon air or carbogen treatment.

\section{PLaQUE REOXYGENATION IMPROVED EFFEROCYTOSIS AND APOPtogIS IN VIVO}

Notably, plaque reoxygenation through daily carbogen exposure was able to prevent necrotic core expansion by $37 \%$ in advanced plaques of the aortic root compared to control, while plaque size was not altered (Figure 3A). Necrotic core expansion is determined by the balance between apoptosis and the clearance of apoptotic cells by phagocytes, a process called efferocytosis. Efferocytosis was quantified in situ, as the ratio of macrophage-bound vs. free apoptotic cells in a TUNEL/MAC3 double immunohistochemistry $(28,29)$. Indeed, carbogen exposure reduced the density of apoptotic cells in the plaque in situ by $50 \%$ and improved efferocytosis of apoptotic cells by $\mathrm{MAC}^{+}$macrophages by almost $40 \%$ compared to air (Figure 3B,C). Increased efferocytosis was seen despite similar plaque macrophage content, size and density (Figure 2E, F). Intraplaque proliferation, collagen content, T cell and total cell density as well as tissue factor (TF) expression as a measure of thrombogenicity (30) were unaffected by carbogen (Figure 3E-H). In initial plaques of the aortic arch, carbogen did not affect plaque size or macrophage content (Supplemental Figure 4), despite successful reoxygenation (Figure 2D). 
Figure 3

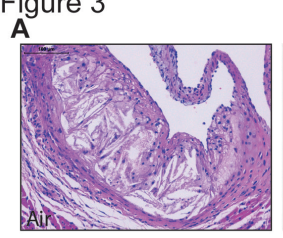

B

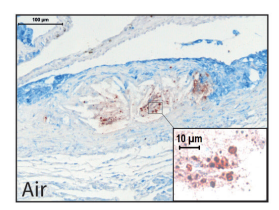

D

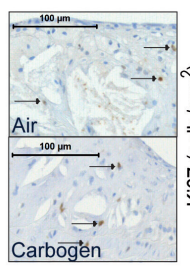

$\mathbf{F}$

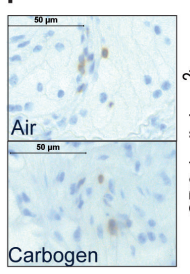

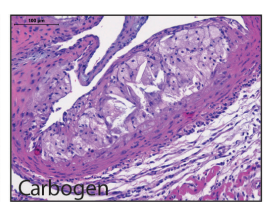

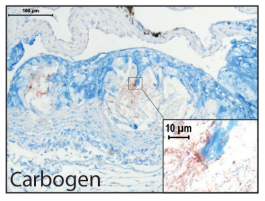

$E$

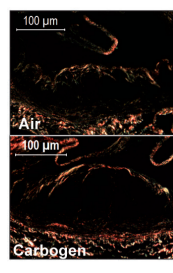

Air Carbogen

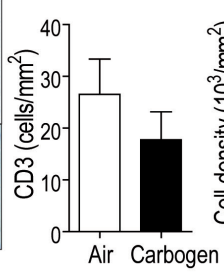

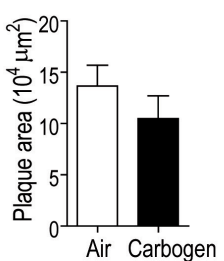
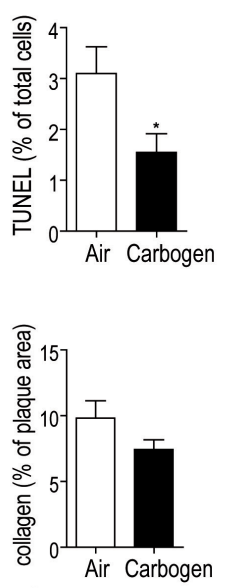

G

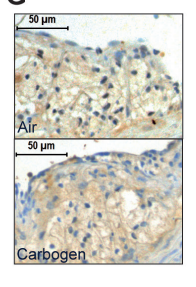

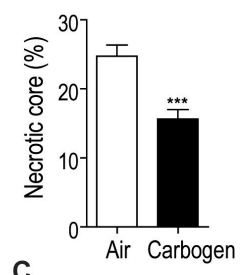

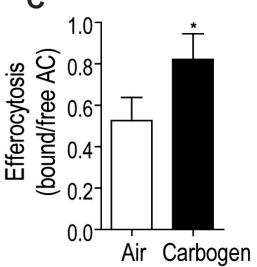

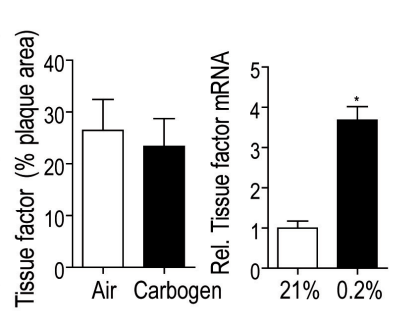

Figure 3: Plaque reoxygenation prevented necrotic core expansion and stimulated efferocytosis in vivo.

A. Hematoxylin and eosin staining and quantifications of aortic root plaque size (left graph) and necrotic core content (right graph) of male LDLr/- mice treated with air or carbogen and fed a HCD for 8 weeks. ***p-value $<0.001 \mathrm{vs.}$ air. B. TUNEL and MAC3 double immunobistochemistry and weak counterstain with hematoxylin (1:50) of $L D L r^{-}$aortic roots treated with air or carbogen. Insets show free TUNEL ${ }^{+}$nuclei (brick red) in air treated group, while TUNEL $L^{+}$nuclei were associated with macrophages (blue) in carbogen treated group. Graphs represent quantification of apoptosis, and C. efferocytosis (ratio MAC3-bound/free TUNEL ${ }^{+}$cells), ${ }^{*} p$-value $<0.05$ vs. air D. Quantification of proliferation (Ki67) expression E. collagen content, F. T cell density and total cell density, as well as G. tissue factor expression, as a measure for thrombogenicity, in aortic roots of $L D L r^{-1}$ mice after chronic air or carbogen exposure (left graph) and in BMDM exposed to 21 or $0.2 \% \mathrm{O}_{2}$ (right graph). 
NEITHER ROS FORMATION, NOR MACROPHAgE POLARIZATION EXPLAIN hyPOXIC REgREgSION OF EFFEROCYTOSIS

Generation of reactive oxygen species (ROS) during carbogen-induced reoxygenation might be involved in apoptosis or efferocytosis. However, neither ROS-mediated DNA damage, analyzed by 8 -hydroxy-2'deoxy-guanosine ( $8 \mathrm{OH}-\mathrm{dG}$ ) immunohistochemistry, nor anti-oxidant capacity of the plasma was changed after chronic (Figure 4A) and single carbogen exposure (not shown). Additionally, hypoxia did not alter macrophage ROS activity in vitro (Figure 4A).

Apoptosis and efferocytosis were further studied in vitro. Chronic hypoxia $\left(0.2 \% \mathrm{O}_{2}\right)$ did not stimulate apoptosis in murine primary BMDM (Figure 4B) or J774 (data not shown) as single stimulus, nor augment apoptosis of additional ER-stress stimuli such as 7-ketocholesterol or tunicamycin. In fact, cells even seemed to be protected from ER-stress mediated apoptosis (Figure 4B). Efferocytosis of labeled, apoptotic Jurkat T-cells by both the J774 murine macrophage cell line and BMDM was hampered in hypoxia $\left(0.2 \% \mathrm{O}_{2}\right)$ compared to $21 \% \mathrm{O}_{2}$ control (Figure $\left.4 \mathrm{C}\right)$. Thus, hypoxia hampered efferocytosis in vitro.

Since anti-inflammatory M2c macrophages are thought to be more efficient in phagocytosis/efferocytosis (31), the effect of hypoxia on polarization was studied. Hypoxia reduced M2 gene expression (interleukin 10 (IL-10) and mannose receptor (MR)), whereas M1 genes (IL-6 and inducible nitric oxide synthase) were strongly upregulated (Figure 4D). In vivo, unlike iNOS, $\mathrm{MR}^{+}$macrophages were hardly present in the plaque; therefore adventitial $\mathrm{MR}^{+}$cells were quantified. A trend towards less M1 in the plaque and more M2 expression in the adventitia of carbogen treated mice was found (Figure 4E-F). Additionally, plasma cytokine levels were essentially not changed, with a trend towards less IL-6 in the carbogen treated group (Figure 4G).

Next, it was investigated if M2 polarization prior to hypoxia could rescue hypoxiamediated repression of efferocytosis. Under normoxic conditions M2c polarization enhanced macrophage efferocytosis capacity compared to unpolarized BMDM, and, unexpectedly, so did M1 polarization (Figure 4H). However, polarization to M2c or M1 was not able to restore the repression of efferocytosis by hypoxia, suggesting that macrophage polarization is not involved in oxygen-dependent regulation of efferocytosis. 
Figure 4
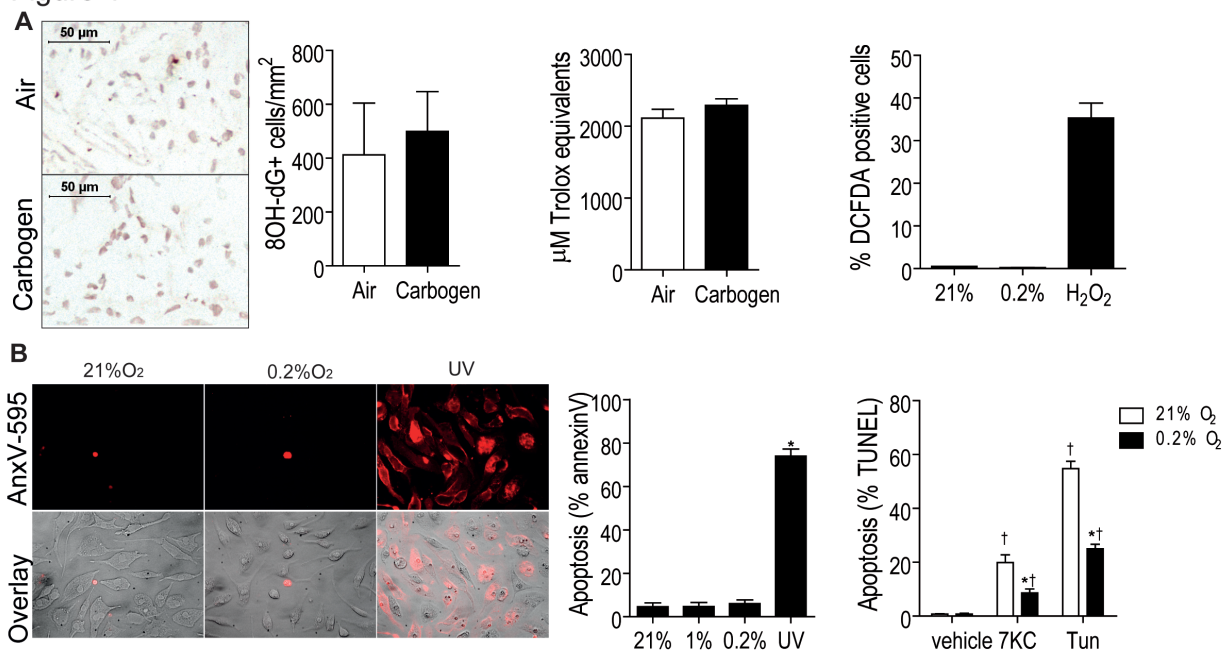

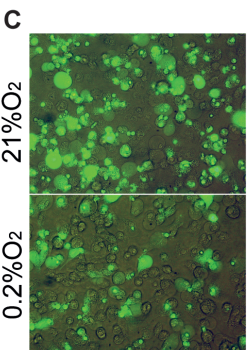

E

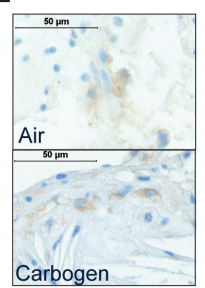

G

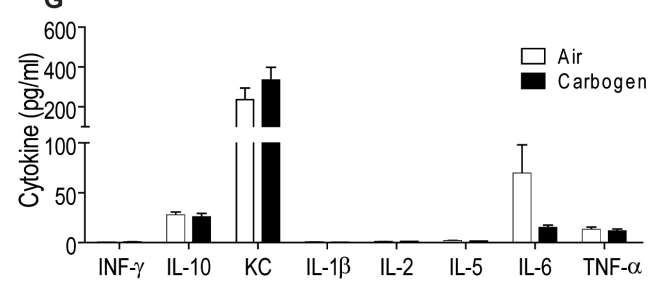

D

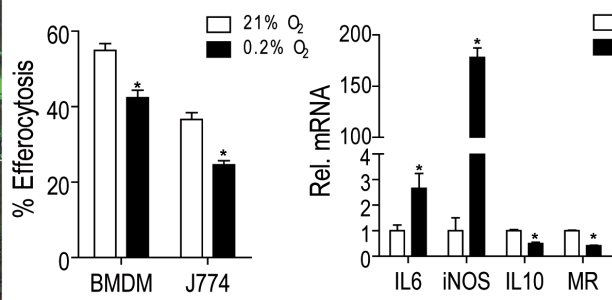

F
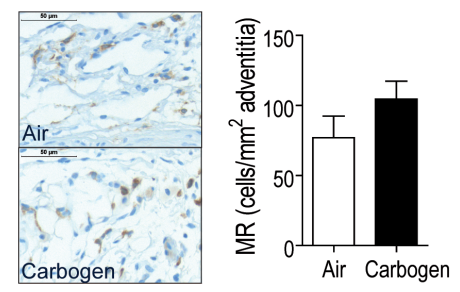

H

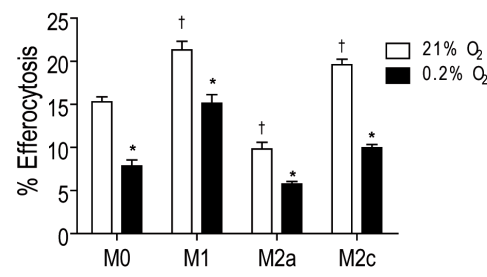

Figure 4: Neither ROS formation, nor macrophage polarization explain hypoxic repression of efferocytosis. 
A. Representative images and quantifications of ROS-mediated DNA damage $(8 \mathrm{OH}-d G)$ in aortic roots of carbogen or air-treated mice ( $n=15 /$ group, left panel), LDL $r^{/-}$plasma anti-oxidant capacity relative to the standard trolox, a water-soluble vitamin $E$ analogue (trolox equivalents, middle graph), and DCFDA incorporation in BMDM exposed to $21 \%, 0.2 \% \mathrm{O}_{2}$ (right graph) for 24hours. B. Representative images and quantifications of percentage of annexin A5 (red) apoptotic BMDM exposed to $21 \% \mathrm{O}_{2}, 0.2 \% \mathrm{O}_{2}$ or UV-irradiated (left graph). Quantification of apoptosis (\% TUNEL) in 7 ketocholesterol and tunicamycin treated BMDM in $21 \% \mathrm{O}_{2}$ or $0.2 \% \mathrm{O}_{2}{ }^{*}{ }^{*}$-value $<0.05$ vs. normoxia, $\uparrow p$-value $<0.05$ vs. normoxia vehicle. $C$. Representative images of calcein-AM labeled, apoptotic Jurkat $T$ cells (green fluorescent) ingested by BMDM (phase contrast overlay with fluorescence) in $21 \% \mathrm{O}_{2}$ and $0.2 \% \mathrm{O}_{2}$. Quantification shows percentage of calcein ${ }^{+} B M D M$ or $J 774$ macrophages having ingested labeled, apoptotic Jurkat $T$ cells. ${ }^{*}$-value <0.05. D. M1 (IL6 - interleukin 6; iNOS - inducible nitric oxide synthase) and M2 (IL10; MR mannose receptor) gene expression of BMDM exposed to $21 \%$ or $0.2 \% \mathrm{O}_{2}$. ${ }^{*}$-value $<0.05 \mathrm{E}$. $i \mathrm{NOS}$ in plaque and adventitia and F. MR expression in the adventitia of aortic roots after chronic carbogen compared to air. G. Plasma cytokine expression in carbogen and air-treated mice $H$. Efferocytosis of apoptotic Jurkats by normoxic or bypoxic unpolarized (MO) BMDM orpolarized towards an M1, M2a or M2c phenotype. * ${ }^{*}$-value $<0.05$ vs. normoxia of same polarization state, $\uparrow p$-value $<0.05$ vs. normoxic $M 0$.

\section{HYPOXIA LIMITS EFFEROCYTOSIS VIA MERTK}

Efferocytosis is a receptor-mediated process. Therefore, we studied whether hypoxia affects expression of known efferocytosis receptors. Indeed, expression of the efferocytosis receptor Mer tyrosine kinase (Mer'TK) and Cluster of Differentiation 36 (CD36) was reduced in hypoxic BMDM, whereas that of Scavenger receptor A (SRA) was unchanged. In contrast, low density lipoprotein receptor-related protein 1 (LRP1) was upregulated (Figure 5A). As efferocytosis capacity was decreased upon hypoxia and LRP-1 was previously not involved in efferocytosis (32), LRP1 is likely not involved.

Compromised function of MerTK was seen to underlie the impaired efferocytosis during murine atherogenesis in vivo $(17,29)$. As expected, disrupted MerTK signaling in MerTK kinase domain deficient $\left(\mathrm{Mer}^{\mathrm{T}} \mathrm{K}^{\mathrm{kd}}\right.$ ) BMDM led to repressed efferocytosis in normoxia (Figure 5B). Importantly, while hypoxia significantly reduced the efferocytosis capacity of WT BMDM, hypoxia was not able to repress efferocytosis in MerTK ${ }^{\mathrm{kd}}$ BMDM any further (Figure 5B). This effect was specifically mediated by the Mer'TK ${ }^{\mathrm{kd}}$, since expression of other efferocytosis receptor expression in Mer'TK $^{\mathrm{kd}} \mathrm{BMDM}$ was unaltered, except for a reduction in CD36 mRNA (Figure 5C). However, as CD36 deficiency did not affect efferocytosis (Figure 5D), as reported previously (32), this suggests that hypoxic repression of efferocytosis in W'T BMDM is no longer effective in MerTK ${ }^{\mathrm{kd}}$ BMDM. Hypoxia-dependent downregulation of MerTK in WT is thus likely responsible for repression of efferocytosis in a hypoxic milieu, such as the atherosclerotic plaque. 
Figure 5
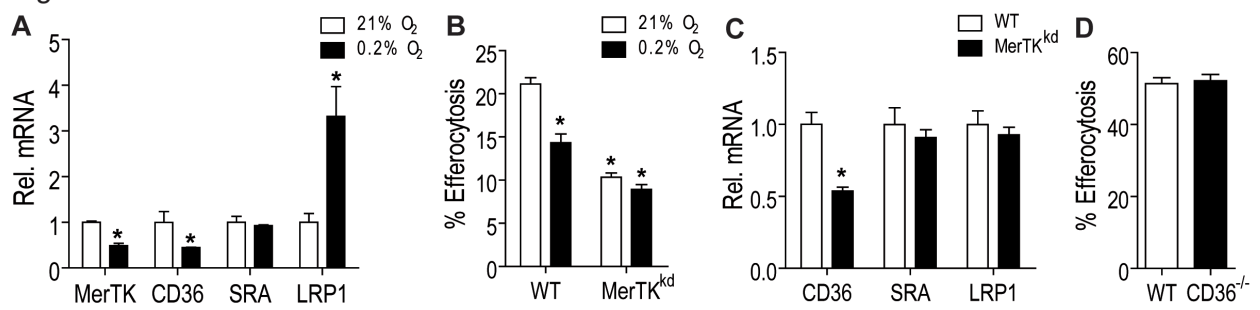

Figure 5: Hypoxia limits efferocytosis via MerTK

A. Efferocytosis receptor gene expression (SRA - Scavenger receptor A; LRP1 - Low density lipoprotein receptorrelated protein 1) in normoxia or $0.2 \% \mathrm{O}_{2}{ }^{*}$-v-value $<0.05$ vs. normoxia of same gene. B. Efferocytosis in wildtype and MerTK ${ }^{k d}$ BMDM exposed to $21 \%$ or $0.2 \% \mathrm{O}_{2}{ }^{*}{ }^{*}$-value $<0.05$ vs. wildtype normoxia. C. Efferocytosis receptor gene expression in wt and MerTK ${ }^{k d} B M D M s$. *p-value $<0.05$ vs. wt of same gene. D. Efferocytosis of apoptotic Jurkats by wt and CD36- BMDM.

\section{DISCUSSION}

This study revealed that reversal of plaque hypoxia can inhibit the progression of atherosclerotic plaques to a vulnerable phenotype, by reducing the development of the plaque-destabilizing necrotic core. Also, it was shown that murine plaque macrophages are hypoxic from the initiation of atherosclerosis on, using the hypoxia-specific marker pimonidazole. Importantly, we revealed that hyperoxic carbogen gas not only achieved reoxygenation of atherosclerotic plaques, liver and spleen in $\mathrm{LDLr}^{-/}$mice, but also prevented apoptotic cell accumulation in the plaque.

Apoptosis induction in vitro was not augmented by hypoxia, suggesting that reoxygenation restored the known deficiency in apoptotic cell clearance in atherosclerosis (28). Mechanistically, hypoxia-dependent transcriptional downregulation of MerTK, and subsequent reduction of apoptotic cell clearance by MerTK is the likely underlying cause for hampered efferocytosis by hypoxic macrophages. Thus, this study is the first to show a causal role of hypoxia in plaque destabilization, specifically in efferocytosis.

In addition, plaque thrombogenicity was studied using TF expression, a key trigger of the extrinsic coagulation system causally linked to plaque thrombosis (30). Interestingly, hypoxia enhanced macrophage expression of TF, supporting the prior correlation of human plaque hypoxia to intraplaque hemorrhage (6). Nevertheless, carbogen did not prevent the hypoxia-induced expression of plaque TF. 
The oxygen debt in atherosclerosis is thought to arise from the sizeable oxygenconsuming metabolism of inflammatory cells, as even macrophages of $\mathrm{LDLr}^{-/}$fatty streaks directly adjacent to arterial oxygen supply, within the 100-200 $\mu$ m oxygen diffusion distance, were hypoxic. This was already suggested by our previous work, showing that human plaque macrophages were hypoxic, despite the close proximity - within $40 \mu \mathrm{m}-$ of arterial blood oxygen supply (6). Moreover, hypoxia was also detected in most tissueresident leukocytes of lymphoid organs in $\mathrm{LDLr}^{-/}$mice. Not surprisingly, as hypoxia was found in many, if not all tissues with an extensive inflammatory influx, such as wound healing, obesity, rheumatoid arthritis, and tumors (33-36). As pimonidazole only forms adducts in low oxygen concentrations $(<10 \mathrm{mmHg}$ oxygen (21)), this indicates that hypoxia in plaque macrophage arises from a high oxygen demand exceeding oxygen supply. Indeed, activated macrophages, in need of ATP for protein production and migration, show 9-fold enhancement of their oxygen consumption (37). Oxygen usage of activated macrophages would then compare to the notoriously high $\mathrm{O}_{2}$ consumption by cardiac myocytes (38) which were indeed also positive for pimonidazole and sensitive to carbogen-mediated reoxygenation (data not shown). Therefore, it is highly likely that upon activation, macrophages rapidly become hypoxic, even within the oxygen-diffusion limit.

Once hypoxic, macrophages will primarily rely on glycolysis as main energy supply, as suggested by a hypoxia-dependent increase in glucose uptake (39). Together, this may explain prior results of plaques with low glucose and ATP content, and high lactate levels (40). Increased glycolytic activity has been linked with M1 polarization of macrophages (41). Indeed, hypoxic macrophages presented with a pro-inflammatory M1 phenotype in vitro, although reoxygenation in vivo failed to switch polarization. Nevertheless, tumorassociated macrophages located in hypoxic tumor regions, express an M2-like profile, and both M1 and M2 marker expression was found in hypoxic adipose tissue macrophages $(42,43)$. Hence, macrophage subtype distinction might not be as clear in vivo, and despite hypoxia, the microenvironment seems to influence macrophage polarization/function also in the plaque. M2c macrophages are the most superior efferocytic macrophage subtype in humans. (31). However, neither M1 nor M2 polarization could protect from hypoxia-mediated reduction in efferocytosis. Mechanistically, low oxygen tension downregulated MerTK and CD36 expression, while upregulating LRP1. Both CD36 and LRP were not involved in efferocytosis in vitro as shown by us and others (32). Hypoxic upregulation of LRP1 expression may be efferocytosis-independent, as LRP1 has a myriad of other functions related to atherosclerosis (44). Only MerTK ${ }^{\mathrm{kd}}$ mimicked the reduced efferocytosis seen in hypoxic macrophages. In fact, MerTK ${ }^{\mathrm{kd}}$ has been shown to aggravate atherosclerosis in mice and worsen lupus-like autoimmunity $(17,29,45)$, 
suggesting a role of MerTK in chronic inflammatory diseases.

While MerTK ${ }^{\mathrm{kd}}$ bone marrow accelerated both atherosclerotic plaque size, as well as necrotic core size (17), carbogen treatment only affected the latter. This may be explained by the potentially partial inhibition of MerTK expression, the intermittent nature of the carbogen treatment and treatment onset after initiation of atherosclerosis. While MerTK ${ }^{\mathrm{kd}}$ transplanted LDLr ${ }^{-/}$recipient mice presented with a continuous and complete absence of MerTK activity prior to plaque initiation (17), normoxic macrophages in vitro show a 50\% increase in Mer'TK expression compared to hypoxic macrophages. Treatment of MerTK ${ }^{\mathrm{kd}}$ mice with carbogen gas could provide definite proof of the MerTK-mediated carbogen effect.

The current study is limited by the systemic nature of carbogen exposure. Therefore, many aspects of peripheral processes instrumental in atherogenesis were examined. Carbogen did not affect erythropoiesis, myelopoiesis, thrombogenicity or plasma cholesterol level. Also, blood pressure and heart rate were unaffected $(46,47)$. Although unlikely, we cannot fully exclude an indirect effect of carbogen on atherogenesis.

Our results unveil an exciting new process to exploit for therapeutic strategies for atherosclerosis. In mice, carbogen gas was able to stabilize the atherosclerotic plaque; if translatable to the human situation, this may potentially prevent plaque ruptures and thrombosis. Indeed, carbogen and hyperbaric oxygen therapy were safely and successfully applied in cancer and diabetic foot patients, respectively $(48,49)$. While in humans with sleep apnea, maintained oxygen supply through continuous positive airway pressure therapy already proved effective in reversing sub-clinical atherosclerosis (50), effectiveness in existing human atherosclerosis remains unclear. Additionally, currently developed tools for imaging of hypoxia $(13,51)$ can be used to monitor the efficacy of carbogen to alter human plaque hypoxia and stability.

\section{CONCLUSION}

In conclusion, carbogen-mediated reoxygenation supports the role of plaque hypoxia as a driver of plaque instability through dysfunctional Mer'TK-mediated clearance of apoptotic cells and subsequent necrotic core expansion. Nevertheless, translation of carbogen therapy to human atherosclerosis will need to be confirmed using non-invasive imaging of plaque hypoxia and stability. 


\section{FUNDING}

Research was supported by a VENI fellowship of the Netherlands Organization of Scientific research (to J.C.S. 016.116.017), a PhD-student fellowship from the Cardiovascular Research Institute Maastricht (to T.T.), a Dr. E. Dekker grant from the Dutch Heart Foundation (M.M.D. 2007T034 and 2012T079) NIH grant HL084312 (to E.A.F.). 


\section{REFERENCES}

1. Carmeliet P. Angiogenesis in life, disease and medicine. Nature. 2005;438(7070):932-6.

2. Fong CC, Zhang Q, Shi YF, Wu RS, Fong WF, Yang M. Effect of hypoxia on RAW264.7 macrophages apoptosis and signaling. Toxicology. 2007;235(1-2):52-61.

3. Hansson GK. Inflammation, atherosclerosis, and coronary artery disease. N Engl J Med. 2005;352(16):1685-95.

4. Tabas I. Macrophage death and defective inflammation resolution in atherosclerosis. Nat Rev Immunol. 2010 Jan;10(1):36-46.

5. Virmani R, Burke AP, Farb A, Kolodgie FD. Pathology of the unstable plaque. Prog Cardiovasc Dis. 2002;44(5):349-56.

6. Sluimer JC, Gasc J-M, van Wanroij JL, Kisters N, Groeneweg M, Sollewijn Gelpke MD, et al. Hypoxia, hypoxia-inducible transcription factor, and macrophages in human atherosclerotic plaques are correlated with intraplaque angiogenesis. J Am Coll Cardiol. 2008 Apr 1;51(13):1258-65.

7. Sluimer JC, Daemen MJ. Novel concepts in atherogenesis: angiogenesis and hypoxia in atherosclerosis. J Pathol. 2009 May;218(1):7-29.

8. Bjornheden T, Levin M, Evaldsson M, Wiklund O. Evidence of Hypoxic Areas Within the Arterial Wall In vivo. Arterioscler Thromb Vasc Biol. 1999 Apr 1;19(4):870-6.

9. Ramkhelawon B, Yang Y, van Gils JM, Hewing B, Rayner KJ, Parathath S, et al. Hypoxia Induces Netrin-1 and Unc5b in Atherosclerotic Plaques: Mechanism for Macrophage Retention and Survival. Arterioscler Thromb Vasc Biol. 2013 Apr 18;33(6):1180-8.

10. Parathath S, Mick SL, Feig JE, Joaquin V, Grauer L, Habiel DM, et al. Hypoxia is present in murine atherosclerotic plaques and has multiple adverse effects on macrophage lipid metabolism. Circ Res. 2011 Oct 28;109(10):1141-52.

11. Murdoch C, Muthana M, Lewis CE. Hypoxia regulates macrophage functions in inflammation. J Immunol. 2005 Nov 15;175(10):6257-63.

12. Burke B, Giannoudis A, Corke KP, Gill D, Wells M, Ziegler-Heitbrock L, et al. Hypoxiainduced gene expression in human macrophages: implications for ischemic tissues and hypoxiaregulated gene therapy. Am J Pathol. 2003;163(4):1233-43.

13. Dubois LJ, Lieuwes NG, Janssen MH, Peeters WJ, Windhorst AD, Walsh JC, et al. Preclinical evaluation and validation of $[18 \mathrm{~F}] \mathrm{HX} 4$, a promising hypoxia marker for PET imaging. Proc Natl Acad Sci U S A. 2011/08/30 ed. 2011;108(35):14620-5.

14. Jordan BF, Magat J, Colliez F, Ozel E, Fruytier AC, Marchand V, et al. Mapping of oxygen by imaging lipids relaxation enhancement: A potential sensitive endogenous MRI contrast to map variations in tissue oxygenation. Magn Reson Med. 2012/10/02 ed. 2012;70(3):732-44.

15. van Laarhoven HW, Bussink J, Lok J, Verhagen I, Punt CJ, Heerschap A, et al. Modulation of hypoxia in murine liver metastases of colon carcinoma by nicotinamide and carbogen. Radiat Res. 2005/09/03 ed. 2005;164(3):245-9.

16. Kaanders JH, Bussink J, van der Kogel AJ. ARCON: a novel biology-based approach in radiotherapy. Lancet Oncol. 2002/12/11 ed. 2002;3(12):728-37.

17. Ait-Oufella H, Pouresmail V, Simon T, Blanc-Brude O, Kinugawa K, Merval R, et al. Defective mer receptor tyrosine kinase signaling in bone marrow cells promotes apoptotic cell accumulation and accelerates atherosclerosis. Arterioscler Thromb Vasc Biol. 2008 Aug;28(8):1429-31.

18. Scott RS, McMahon EJ, Pop SM, Reap EA, Caricchio R, Cohen PL, et al. Phagocytosis and clearance of apoptotic cells is mediated by MER. Nature. 2001/05/11 ed. 2001;411(6834):207-11.

19. Febbraio M, Abumrad NA, Hajjar DP, Sharma K, Cheng W, Pearce SF, et al. A null mutation in murine CD36 reveals an important role in fatty acid and lipoprotein metabolism. J Biol Chem. 1999 Jul 2;274(27):19055-62.

20. Janssen B, Debets J, Leenders P, Smits J. Chronic measurement of cardiac output in conscious 
mice. Am J Physiol Regul Integr Comp Physiol. 2002/02/08 ed. 2002;282(3):R928-35.

21. Chou SC, Azuma Y, Varia MA, Raleigh JA. Evidence that involucrin, a marker for differentiation, is oxygen regulated in human squamous cell carcinomas. Br J Cancer. 2004/02/05 ed. 2004;90(3):728-35.

22. Kleiter MM, Thrall DE, Malarkey DE, Ji X, Lee DY, Chou SC, et al. A comparison of oral and intravenous pimonidazole in canine tumors using intravenous CCI-103F as a control hypoxia marker. Int J Radiat Oncol Biol Phys. 2006;64(2):592-602.

23. Schrijvers DM, De Meyer GRY, Kockx MM, Herman AG, Martinet W. Phagocytosis of apoptotic cells by macrophages is impaired in atherosclerosis. Arterioscler Thromb Vasc Biol. 2005 Jun;25(6):1256-61.

24. Thorp E, Cui D, Schrijvers DM, Kuriakose G, Tabas I. Mertk receptor mutation reduces efferocytosis efficiency and promotes apoptotic cell accumulation and plaque necrosis in atherosclerotic lesions of apoe-/- mice. Arterioscler Thromb Vasc Biol. 2008 Aug;28(8):1421-8.

25. Fischer MA, Gransier TJ, Beckers LM, Bekers O, Bast A, Haenen GR. Determination of the antioxidant capacity in blood. Clin Chem Lab Med. 2005/10/07 ed. 2005;43(7):735-40.

26. Sluimer JC, Kisters N, Cleutjens KB, Volger OL, Horrevoets AJ, van den Akker LH, et al. Dead or Alive: Gene expression profiles of advanced atherosclerotic plaques from autopsy and surgery. Physiol Genomics. 2007;30:335-41.

27. Seijkens T, Hoeksema MA, Beckers L, Smeets E, Meiler S, Levels J, et al. Hypercholesterolemiainduced priming of hematopoietic stem and progenitor cells aggravates atherosclerosis. FASEB J. 2014 May;28(5):2202-13.

28. Schrijvers DM, De Meyer GRY, Kockx MM, Herman AG, Martinet W. Phagocytosis of apoptotic cells by macrophages is impaired in atherosclerosis. Arterioscler Thromb Vasc Biol. 2005 Jun;25(6):1256-61.

29. Thorp E, Cui D, Schrijvers DM, Kuriakose G, Tabas I. Mertk receptor mutation reduces efferocytosis efficiency and promotes apoptotic cell accumulation and plaque necrosis in atherosclerotic lesions of apoe-/- mice. Arter Thromb Vasc Biol. 2008;28(8):1421-8.

30. Borissoff JI, Heeneman S, Kilinç E, Kassák P, Van Oerle R, Winckers K, et al. Early atherosclerosis exhibits an enhanced procoagulant state. Circulation. 2010 Aug 24;122(8):821-30.

31. Zizzo G, Hilliard BA, Monestier M, Cohen PL. Efficient Clearance of Early Apoptotic Cells by Human Macrophages Requires M2c Polarization and MerTK Induction. J Immunol. 2012 Aug 31;189(7):3508-20.

32. Li Y, Gerbod-Giannone M-C, Seitz H, Cui D, Thorp E, Tall AR, et al. Cholesterol-induced apoptotic macrophages elicit an inflammatory response in phagocytes, which is partially attenuated by the Mer receptor. J Biol Chem. 2006 Mar 10;281(10):6707-17.

33. Kennedy AS, Raleigh JA, Perez GM, Calkins DP, Thrall DE, Novotny DB, et al. Proliferation and hypoxia in human squamous cell carcinoma of the cervix: first report of combined immunohistochemical assays. Int J Radiat Oncol Biol Phys. 1997;37(4):897-905.

34. Lee YA, Kim JY, Hong SJ, Lee SH, Yoo MC, Kim KS, et al. Synovial proliferation differentially affects hypoxia in the joint cavities of rheumatoid arthritis and osteoarthritis patients. Clin Rheumatol. 2007;26(12):2023-9.

35. Haroon ZA, Raleigh JA, Greenberg CS, Dewhirst MW. Early wound healing exhibits cytokine surge without evidence of hypoxia. Ann Surg. 2000;231(1):137-47.

36. Rausch ME, Weisberg S, Vardhana P, Tortoriello D V. Obesity in C57BL/6J mice is characterized by adipose tissue hypoxia and cytotoxic T-cell infiltration. Int J Obes. 2008;32:451-63.

37. Rist RJ, Naftalin RJ. Glucose- and phorbol myristate acetate-stimulated oxygen consumption and superoxide production in rat peritoneal macrophages is inhibited by dexamethasone. Biochem J. 1993/04/15 ed. 1993;291 ( Pt 2:509-14.

38. Wagner BA, Venkataraman S, Buettner GR. The rate of oxygen utilization by cells. Free 
Radic Biol Med. 2011/06/15 ed. 2011;51(3):700-12.

39. Folco EJ, Sheikine Y, Rocha VZ, Christen T, Shvartz E, Sukhova GK, et al. Hypoxia but not inflammation augments glucose uptake in human macrophages: Implications for imaging atherosclerosis with 18fluorine-labeled 2-deoxy-D-glucose positron emission tomography. J Am Coll Cardiol. 2011 Aug 2;58(6):603-14.

40. Levin M, Leppanen O, Evaldsson M, Wiklund O, Bondjers G, Bjornheden T. Mapping of ATP, glucose, glycogen, and lactate concentrations within the arterial wall. Arter Thromb Vasc Biol. 2003;23(10):1801-7.

41. Kawaguchi T, Veech RL, Uyeda K. Regulation of energy metabolism in macrophages during hypoxia. Roles of fructose 2,6-bisphosphate and ribose 1,5-bisphosphate. J Biol Chem. 2001 Jul 27;276(30):28554-61.

42. Fujisaka S, Usui I, Ikutani M, Aminuddin A, Takikawa A, Tsuneyama K, et al. Adipose tissue hypoxia induces inflammatory M1 polarity of macrophages in an HIF- $1 \alpha$-dependent and HIF-1 $\alpha$ independent manner in obese mice. Diabetologia. 2013 Mar 15;56(6):1403-12.

43. Van Overmeire E, Laoui D, Keirsse J, Van Ginderachter JA. Hypoxia and tumor-associated macrophages: A deadly alliance in support of tumor progression. Oncoimmunology. 2014 Jan 1;3(1):e27561.

44. Lillis AP, Van Duyn LB, Murphy-Ullrich JE, Strickland DK. LDL receptor-related protein 1: unique tissue-specific functions revealed by selective gene knockout studies. Physiol Rev. 2008 Jul;88(3):887-918.

45. Cohen PL, Caricchio R, Abraham V, Camenisch TD, Jennette JC, Roubey RAS, et al. Delayed apoptotic cell clearance and lupus-like autoimmunity in mice lacking the c-mer membrane tyrosine kinase. J Exp Med. 2002 Jul 1;196(1):135-40.

46. Brizel DM, Lin S, Johnson JL, Brooks J, Dewhirst MW, Piantadosi CA. The mechanisms by which hyperbaric oxygen and carbogen improve tumour oxygenation. Br J Cancer. 1995 Nov;72(5):1120-4.

47. Kergoat $\mathrm{H}$, Faucher $\mathrm{C}$. Effects of oxygen and carbogen breathing on choroidal hemodynamics in humans. Invest Ophthalmol Vis Sci. 1999/11/05 ed. 1999;40(12):2906-11.

48. Hoskin P, Rojas A, Saunders M. Accelerated radiotherapy, carbogen, and nicotinamide (ARCON) in the treatment of advanced bladder cancer: mature results of a Phase II nonrandomized study. Int J Radiat Oncol Biol Phys. 2009 Apr 1;73(5):1425-31.

49. Lipsky BA, Berendt AR. Hyperbaric oxygen therapy for diabetic foot wounds: has hope hurdled hype? Diabetes Care. 2010 May 1;33(5):1143-5.

50. Drager LF, Bortolotto LA, Figueiredo AC, Krieger EM, Lorenzi GF. Effects of continuous positive airway pressure on early signs of atherosclerosis in obstructive sleep apnea. Am J Respir Crit Care Med. 2007 Oct 1;176(7):706-12.

51. Silvola JMU, Saraste A, Forsback S, Laine VJO, Saukko P, Heinonen SE, et al. Detection of hypoxia by [18F]EF5 in atherosclerotic plaques in mice. Arterioscler Thromb Vasc Biol. 2011 May;31(5):1011-5. 


\section{SUPPLEMENTAL DATA}

\begin{tabular}{|lll|}
\hline Gene & Forward primer & Reverse primer \\
\hline Cyclophylin & CAAATGCTGGACCAAACACAA & TTCACCTTCCCAAAGACCACAT \\
\hline 18S rRNA & GTAACCCGTTGAACCCCATT & CCATCCAATCGGTAGTAGCG \\
\hline IL6 & ACAAGTCGGAGGCTTAATTACACAT & TTGCCATTGCACAACTCTTTTC \\
\hline iNOS & CAGCTGGGCTGTACAAACCTT & CATTGCAAGTGAAGCGTTTCG \\
\hline IL10 & TGCTCCTAGAGCTGCGGACT & TGCTCCTAGAGCTGCGGACT \\
\hline MR & GCAAATGGAGCCGTCTGTGC & CTCGTGGATCTCCGTGACAC \\
\hline MerTK & CACAATGACAAAGGACTGACG & AGTAGCCATCAAAACCAGGG \\
\hline CD36 & GCCAAGCTATTGCGACATGA & AAAAGAATCTCAATGTCCGAGACTTT \\
\hline LRP1 & GGACCACCATCGTGGAAA & TCCCAGCCACGGTGATAG \\
\hline
\end{tabular}

Supplemental table 1: Sequences of quantitative RT-PCR primers

\section{Supplemental figure I}

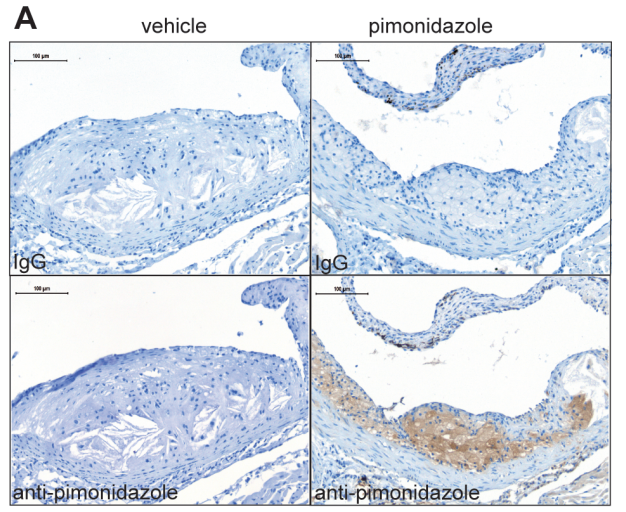

B
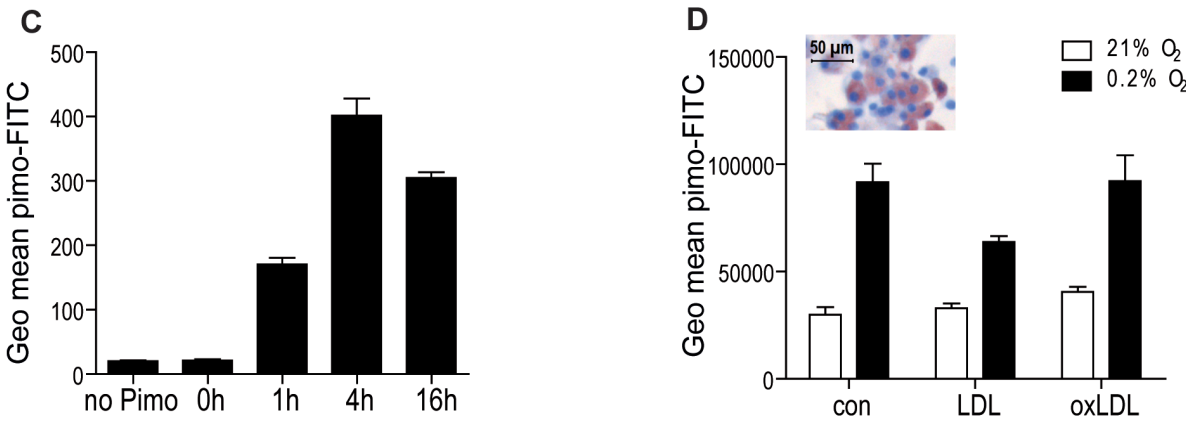

Supplemental Figure 1: Specificity of pimonidazole as hypoxic marker

A. Representative pictures of pimonidazole detection (brown, DAB) in LDL $r^{*-}$ mice receiving pimonidazole injection (right panels) or vebicle (left panels), and immunobistochemistry using anti-pimonidazole antibody (bottom panels) or isotype IgG control (top panels). B. THP-1 macrophages exposed to decreasing oxygen concentrations show increased pimonidazole incorporation. C. Pimonidazole positivity in THP-1 macrophages subjected to $0.2 \%$ oxygen for increasing 
time periods. D. BMDMs treated with LDL, oxLDL or control only show increased pimonidazole incorporation upon exposure to $0.2 \% \mathrm{O}_{2}$ not after incubation in normoxia. Foam cell formation was confirmed with Oil Red O. All graphs present geo mean fuorescence of anti-pimonidazole FITC measured by flow cytometry.

\section{Supplemental figure II}

A

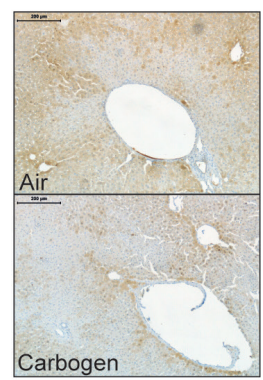

C

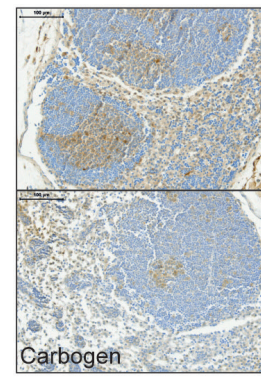

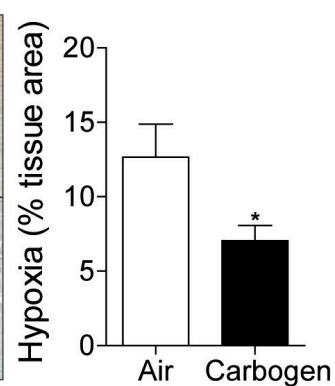

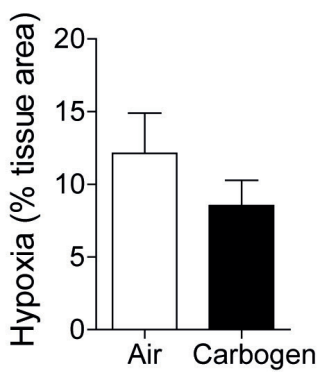

B
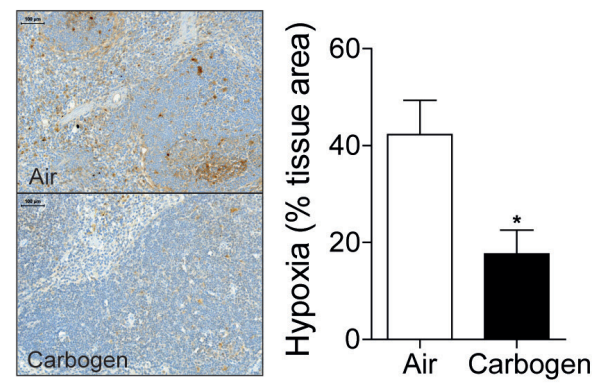

D arterial WBC venous WBC

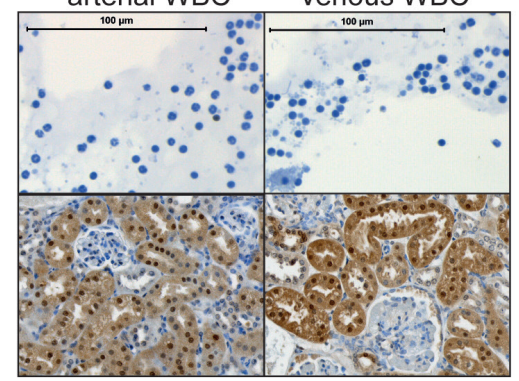

Supplemental Figure 2: Carbogen reoxygenated spleen and lymph nodes

A. Representative pictures and quantifications of liver, B. spleen, and C. lymph node stained with pimonidazole (brown) from air or carbogen treated $L D L r^{\prime}-$ mice. ${ }^{*} p$-value $<0.05$. D. Representative pictures of pimonidazole stained arterial and venous white blood cells of two separate LDLr/- mice, with their respective mouse-matched kidney sections stained as positive control. 


\section{Supplemental figure III}
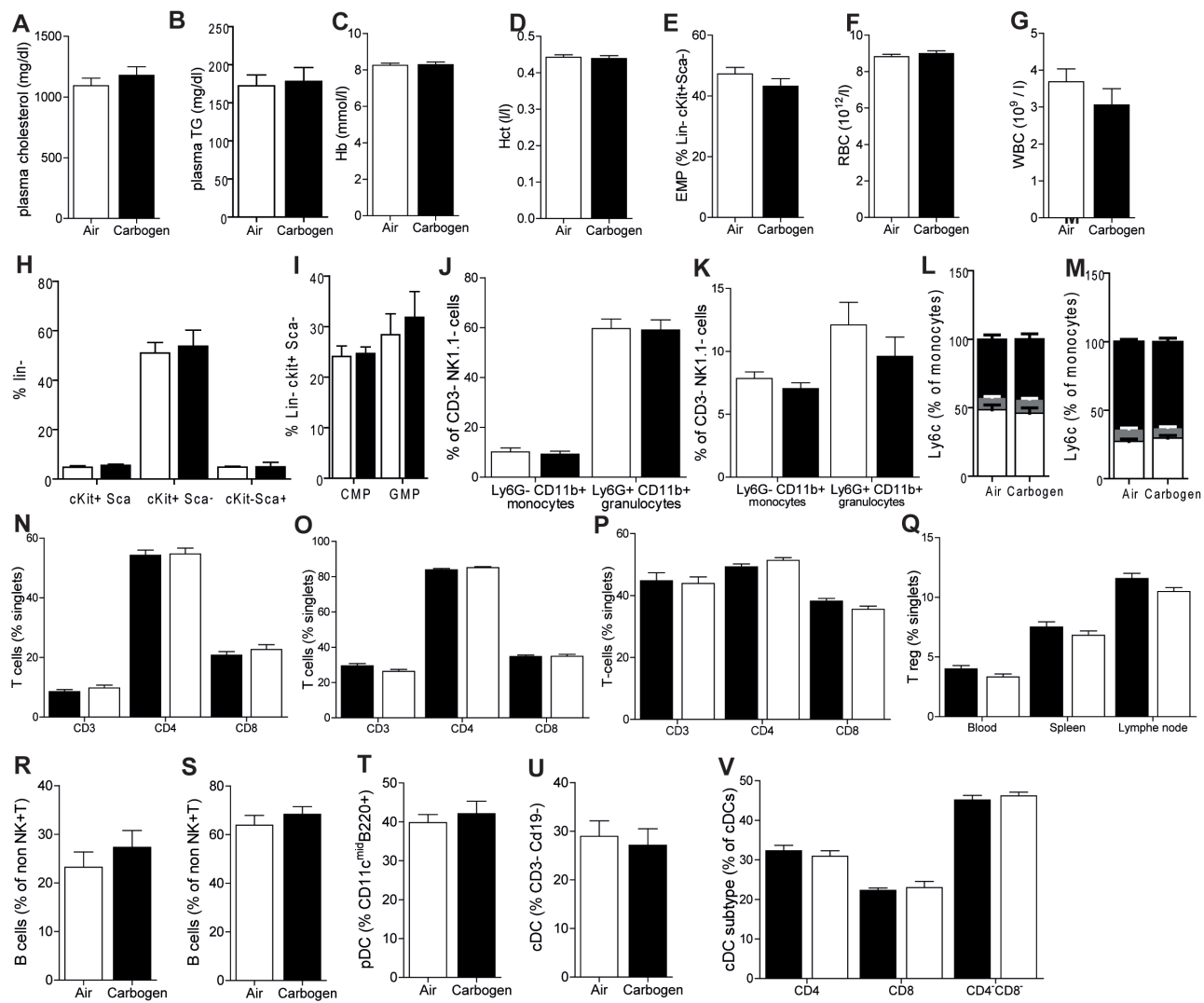

Supplemental Figure 3: Cholesterol and hematopoiesis are unchanged after carbogen exposure.

A. plasma cholesterol, B. plasma triglycerides (TG), C. hemoglobin (Hb), and D. hematocrit (Ht), E. bone marrow erythrocyte-megakaryocyte progenitors (EMP, CD16/32CD34), F. absolute erythrocyte counts and G. absolute white blood cell counts were similar between carbogen (black bars) or air-treated mice (wbite bars). H. Bone marrow stem cells, I. common myeloid progenitor (CMP), and granulocyte-macrophage progenitor (GMP) were similar between carbogen or air-treated mice. J. Blood and K. spleen monocyte and granulocyte populations and L. blood and M. spleen Ly6C monocytic subsets (Ly6C high, white bar; Ly6Clow, grey bar; Ly6C "ug, black bar) were similar between carbogen or airtreated mice N. Total T cells (CD3), T helper (CD4) and cytotoxic T cells (CD8) were similar between carbogen or air-treated mice in blood, O. spleen, and P. lymph nodes. Q. T regulatory cell (T-reg) counts were also similar in blood, spleen and lymph nodes of carbogen or air-treated mice. R. B-cells were similar between carbogen or air-treated mice in blood, and S. spleen. T. Plasmacytoid dendritic cells ( $p D C), U$. conventional $D C(c D C)$ and $V . c D C$ subsets were similar between carbogen or air-treated mice in spleen. 


\section{Supplemental figure IV}
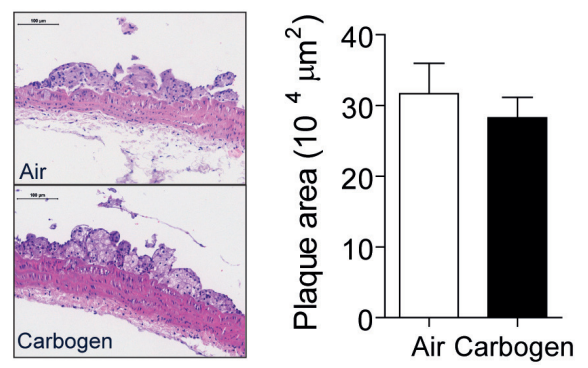

Supplemental Figure 4: Carbogen did not change plaque initiation in the aortic arch.

Representative hematoxylin and eosin images and quantification of aortic arch plaques of $\mathrm{LDLr} \mathrm{r}^{-}$mice exposed to an initial 4 weeks of HCD followed by 4 weeks of diet in combination with air (wbite bars) or carbogen gas (black bars). 



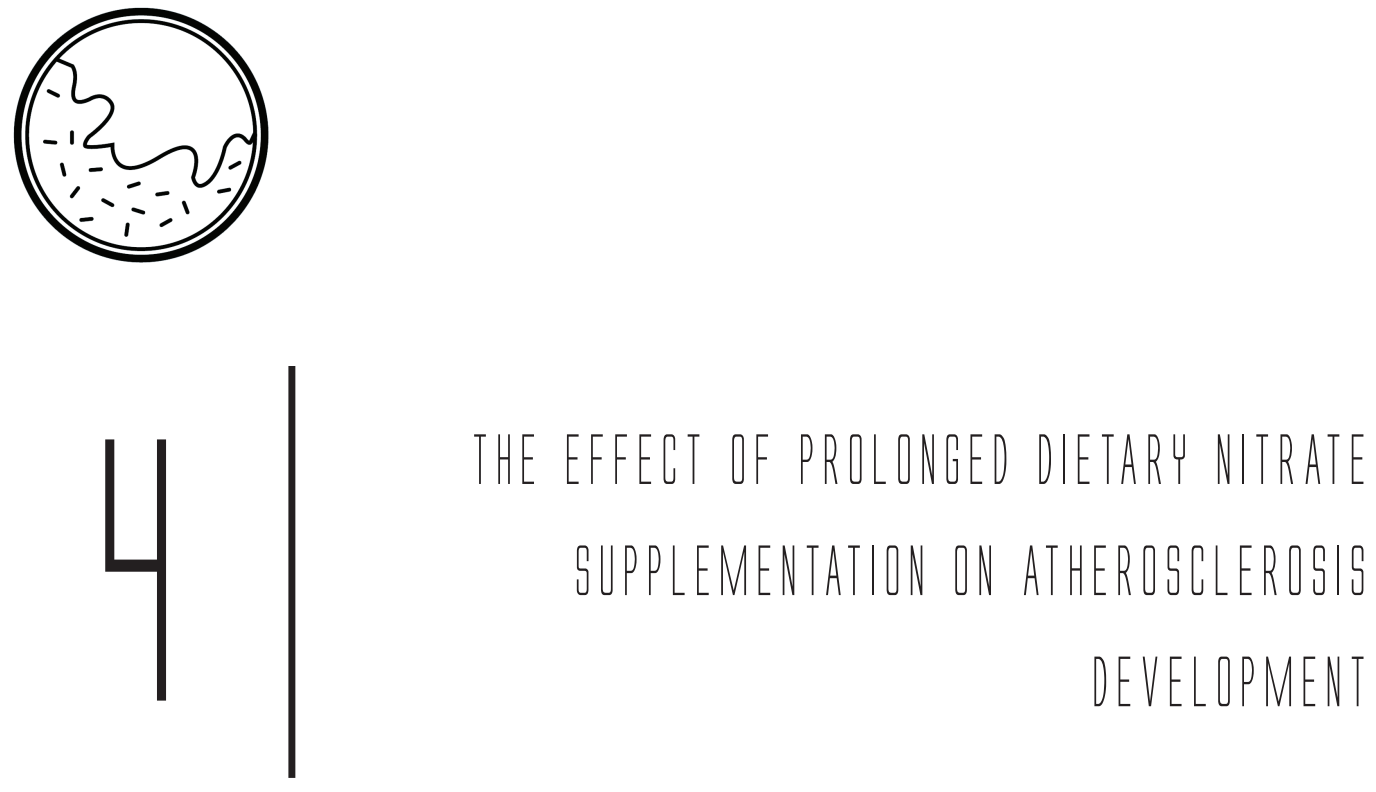

margch e, theelen tL, Jangsen bua, briede dل, haenen gr, genden JMg, van loon lub, poeze M, BIERAU J, GIJBELS MJ, DAEMEN MJAP, gLUIMER JC ATHERDGCLEROSIS 2016 FEB;245:212-21. 



\section{ABSTRACT}

\section{BACKGROUND}

Short term dietary nitrate or nitrite supplementation has nitric oxide (NO)-mediated beneficial effects on blood pressure and inflammation and reduces mitochondrial oxygen consumption, possibly preventing hypoxia. As these processes are implicated in atherogenesis, dietary nitrate was hypothesized to prevent plaque initiation, hypoxia and inflammation.

\section{APPROACH AND RESULTS}

$\mathrm{LDLr}^{-/}$mice were administered sodium-nitrate or equimolar sodium-chloride in drinking water alongside a high cholesterol diet for 14 weeks to induce atherosclerosis. Plasma nitrate, nitrite and hemoglobin-bound nitric oxide were measured by chemiluminescence and electron paramagnetic resonance, respectively.

Plasma nitrate levels were elevated after 14 weeks of nitrate supplementation $(\mathrm{NaCl}$ : $\left.40.29 \pm 2.985, \mathrm{NaNO}_{3}: 78.19 \pm 6.837, \mathrm{p}<0.0001\right)$. However, prolonged dietary nitrate did not affect systemic inflammation, hematopoiesis, erythropoiesis and plasma cholesterol levels, suggesting no severe side effects. Surprisingly, neither blood pressure, nor atherogenesis was altered. Mechanistically, plasma nitrate and nitrite were elevated after two weeks $\left(\mathrm{NaCl}: 1.0 \pm 0.2114, \mathrm{NaNO}_{3}: 3.977 \pm 0.7371, \mathrm{p}<0.0001\right)$, but decreased over time $(6,10$ and 14 weeks). Plasma nitrite levels even reached baseline levels at 14 weeks $\left(\mathrm{NaCl}: 0.7188 \pm 0.1072, \mathrm{NaNO}_{3}: 0.9723 \pm 0.1279, \mathrm{p}=0.12\right)$. Also hemoglobin-bound $\mathrm{NO}$ levels were unaltered after 14 weeks. This compensation was not due to altered eNOS activity or conversion into peroxynitrite and other RNI, suggesting reduced nitrite formation or enhanced nitrate/nitrite clearance.

\section{CONCLUSION}

Prolonged dietary nitrate supplementation resulted in compensation of nitrite and NO levels and did not affect atherogenesis or exert systemic side effects. 


\section{INTRODUCTION}

Ischemic heart disease and stroke remain the leading cause of death in the western world (1). Atherosclerosis presents the main underlying cause of cardiovascular disease. Atherosclerosis is a lipid-driven inflammatory disease, initiated by endothelial dysfunction, resulting in accumulation and subsequent oxidation of cholesterol in the vessel wall. In turn, this triggers inflammatory cell infiltration and macrophage foam cell formation leading to apoptosis and secondary necrosis and plaque advancement (2). Additionally, atherosclerotic plaques are hypoxic (3), with hypoxia playing a causal role in atherogenesis by reducing macrophage efferocytosis capacity (4). In support, sleep apnea patients suffer from an increased risk of atherosclerosis $(5,6)$ and continuous positive airway pressure-mediated oxygen supply in patients can improve endothelial function (7).

Seeing this highly complex disease, we aimed to target atherosclerosis on multiple levels: ameliorating hypertension, hypoxia and inflammation. Inorganic dietary nitrate supplementation may present such a multi-targeted approach, as it has been shown to dampen several processes involved in atherosclerosis: hypertension, hypoxia, endothelial dysfunction and inflammation. Short term dietary nitrate supplementation has been shown to lower resting blood pressure in humans (8). Furthermore, dietary nitrate supplementation improves mitochondrial function, thereby reducing oxygen requirement during exercise (9-11). This reduced cellular oxygen consumption may potentially restore plaque oxygenation and alleviate plaque progression. Additionally, one week dietary nitrate and nitrite supplementation reduced infarct size in a murine myocardial ischemia-reperfusion model, by replenishing nitrate and nitrite stores during infarction (12). Also, dietary nitrate resulted in enhanced cardiorespiratory function (13) and improved glucose tolerance in diabetic rats (14). Nitrate-rich beetroot juice intake prior to exercise achieved similar results, in particular improving exercise tolerance to high-intensity exercise (15), but also in patients with chronic obstructive pulmonary disease (16). Further, an anti-inflammatory effect has been linked to dietary nitrate and nitrite consumption (17), overall hinting towards an atherosclerosis-protective role of dietary nitrate supplementation. Mechanistically, nitrate is reduced to nitrite by commensal bacteria of the gastrointestinal tract and body surfaces (18). The resulting nitrite can be further reduced to nitric oxide (NO) via numerous pathways (reviewed in (19)). In addition to the well-known vasodilatory and anti-hypertensive action of NO $(20,21)$, NO inhibition enhanced leukocyte rolling and adhesion (22). This suggests an anti-atherogenic leukocyte action of $\mathrm{NO}$ itself. Also, NO production rate is decreased in atherosclerosis in patients $(23,24)$ which may contribute to the general pro-inflammatory profile and hampered vasodilatation seen in this disease. Furthermore, nitrate 
supplementation via drinking water reduced triglyceride levels, body weight gain and glucose intolerance in endothelial nitric oxide synthase (eNOS) deficient mice(25), thus reversing symptoms of the metabolic syndrome. This suggests also anti-atherosclerotic effects of nitrate.

Dietary nitrate supplementation has entered clinical trials in cardiovascular disease, however, the results are variable (26-28). Most human and murine studies have applied short nitrate supplementation regimens ranging from 2-6 days to 15 days in humans, reporting many health benefits and improved functional performance (10,29-31). Additionally, conflicting evidence about possible side-effects associated with long term nitrate supplementation (32) has prompted us to study the effects of prolonged dietary nitrate supplementation in hypercholesterolemic low density lipoprotein receptor deficient mice $\left(\mathrm{LDLr}^{-}\right)$. Based on the atheroprotective effects of nitrate described, we hypothesized that prolonged dietary nitrate reverses plaque hypoxia, lowers hypertension and reduces systemic inflammation, thereby alleviating plaque burden.

\section{MATERIAL AND METHODS}

\section{ANIMALS}

All mouse experiments were approved by the regulatory authority of the Maastricht University Medical Centre and performed in compliance with the guidelines described in the directive 2010/63/EU of the European Parliament. All mice were bred at least 9x on C57/JBl6 background and male $\mathrm{LDLr}^{-/}$mice were obtained from an in-house breeding colony, originating from Charles River (Wilmington, MA, USA). All animals were housed in individually ventilated cages (GM500, Techniplast) in groups of up to 5 animals per cage, with bedding (corncob, Technilab-BMI) and cage enrichment. Cages were changed $1 \mathrm{x}$ weekly, reducing handling of the mice to one handling per week during non-intervention periods.

\section{ATHEROSCLEROSIS AND DIETARY NITRATE}

Male LDLr ${ }^{-1}$ mice ( $\mathrm{n}=15$ per group, 9 weeks of age) were fed a high cholesterol diet (HCD, 0.25\% cholesterol, SDS 824171, ad libitum) for 14 weeks and cages with up to 5 mice were randomly assigned to receive either sodium-nitrate $\left(\mathrm{NaNO}_{3}, 1 \mathrm{~g} / \mathrm{L}\right)$ or equimolar sodium-chloride $(\mathrm{NaCl})$ in drinking water ad libitum throughout the course of HCD. A dose of $1 \mathrm{~g} / \mathrm{L} \mathrm{NaNO}_{3}$ equals ca. $150 \mu \mathrm{mol}$ nitrate per day based on observed drinking behavior. This is similar to a vegetable rich diet in humans $(19,33)$. Drinking 
water and HCD contain nitrates; however the levels of nitrates in water and HCD are negligible, as they only account for up to $4.1 \%$ of the total nitrate intake upon nitrate drinking (25mg/ $\mathrm{L} \mathrm{NO}_{3}$ in drinking water (34) and $8 \mathrm{mg} \mathrm{NO} / \mathrm{kg} \mathrm{HCD}$, as analyzed by Special Diet Services). 1 hour prior to sacrifice, mice were intraperitoneally (i.p) injected with the hypoxia-specific marker pimonidazole $(100 \mathrm{mg} / \mathrm{kg}$, hypoxyprobe Omni kit, Hypoxyprobe Inc. Burlington, MA, USA). Mice were euthanized by exsanguination among pentobarbital-anesthesia (100mg/kg i.p).

\section{BLOOD PRESSURE AND HEART RATE MEASUREMENT}

One week prior to sacrifice, systolic, diastolic and mean blood pressure as well as heart rate were measured ( $\mathrm{n}=8$ per group) by tailcuff method using a Volume Pressure Recording sensor (CODA, KENT Scientific Corporation, Torrington, CT, USA) (35).

To confirm the tailcuff measurements, mean arterial pressure (MAP) and heart rate were confirmed in anesthetized mice (Isoflurane; Baxter, Deerfiel, IL, USA), ( $n=4$ per group) at the day of sacrifice. A PE-10 catheter was advanced into the thoracic aorta via the carotid artery and the catheter was subsequently connected to a pressure transducer (Miller Instruments, Houston, TX, USA) with the pressure signal being sampled at 2 $\mathrm{kHz}$. MAP and heart rate were calculated after hemodynamic variables were stabilized.

\section{ATHEROSCLEROSIS QUANTIFICATION AND IMMUNOHISTOCHEMISTRY}

Mice were euthanized with a pentobarbital overdose $(100 \mathrm{mg} / \mathrm{kg}$ i.p.) and blood was withdrawn via the right ventricle for flow cytometry, absolute white and red blood cell count (Coulter Ac. T diff, Beckman Coulter) and total cholesterol analysis. Mice were perfused via the left cardiac ventricle with PBS containing sodium nitroprusside $(0.1 \mathrm{mg} /$ ml; Sigma-Aldrich, Seelze, Germany) followed by 1\% paraformaldehyde (PFA). Aortic arch, root and organs were subsequently excised and fixed in 1\% PFA overnight and paraffin-embedded.

Aortic roots and arches were serially sectioned and stained with hematoxylin and eosin (H\&E, Sigma) for plaque area and lipid core content quantification. Five consecutive $\mathrm{H} \& \mathrm{E}$ sections at $20 \mu \mathrm{m}$ intervals were averaged per mouse and analyzed blindly using computerized morphometry (Leica QWin V3, Cambridge, UK). Sections within this $100 \mu \mathrm{m}$ interval were used for remaining immunohistochemical stainings. For all stainings, antigen retrieval was performed at pH6 (Dako REAL target retrieval, Dako). Hypoxia was detected in the aortic roots using a rabbit polyclonal antibody (clone 2627) 
directed against pimonidazole derivates, formed specifically in hypoxic but living cells in vivo (\% pimonidazole/total plaque area). Additionally, macrophage content of aortic root plaques was quantified as $\mathrm{MAC}^{+}$cells/plaque area (rat anti-mouse MAC-3, clone $\mathrm{M} 3 / 84, \mathrm{BD})$.

\section{TOTAL CHOLESTEROL AND TRIGLYCERIDES}

Plasma was obtained by centrifugation and stored at $-80^{\circ} \mathrm{C}$ until further use. Plasma cholesterol and TG were assessed using standard enzymatic assays (Cholesterol FS'10; Ref: 113009910 021; Diagnostic Systems GmbH, Holzheim, Germany and Triglycerides FS 5' Ecoline; Ref: 157609990 314; Diagnostic Systems GmbH, Holzheim, Germany) automated on the Cobas Fara centrifugal analyzer (Roche).

\section{FLOW CYTOMETRY}

Cells isolated from whole blood, spleen and lymph nodes were analyzed using flow cytometry ( $\mathrm{n}=10$ per group). TruCount absolute cell count tubes (BD Biosciences) were used on whole blood samples, to allow absolute blood cell count. Spleen and lymph nodes were crushed into single-cell suspensions and blood and spleen were subsequently subjected to erythrocyte lysis. Following specific antibodies were used to detect leucocytes subsets in all tissues: leucocytes $\left(\mathrm{CD} 45^{+}\right.$, Biolegend), NK-T cells (CD $3 \varepsilon^{+}$ NK1-1 ${ }^{+}$; Miltenyi, eBioscience, respectively), CDT cells (CD3 $\varepsilon^{+}$NK1-1; Miltenyi, eBioscience, respectively), $\mathrm{T}$ helper cells (CD4 $\left.{ }^{+}, \mathrm{BD}\right)$, cytotoxic $\mathrm{T}$ cells (CD8a $\left.{ }^{+}, \mathrm{BD}\right)$, effector $\mathrm{T}$ cells $\left(\mathrm{CD} 44^{\text {high }}, \mathrm{CD} 62^{\text {low }}\right.$; $\mathrm{BD}$, eBioscience, respectively), central memory $\mathrm{T}$ cells (CD44 $\left.{ }^{\text {high }} \mathrm{CD} 66^{\text {high }}\right)$, naïve $\mathrm{T}$ cells $\left(\mathrm{CD} 44^{\text {low }} \mathrm{CD}^{\text {high }}\right.$ ), regulatory $\mathrm{T}$ cells $\left(\mathrm{CD} 4^{+}\right.$ CD25 ${ }^{\text {high }}$ FoxP $3^{\text {high }}$; eBioscience), B cells $\left(B 220^{+}\right.$; BD), NK cells $\left(\mathrm{NK} 1-1^{+}\right)$, granulocytes $\left(\mathrm{CD} 11 \mathrm{~b}^{\text {high }} \mathrm{Ly} 6 \mathrm{G}^{\text {high }} ; \mathrm{BD}\right.$, eBioscience, respectively) and monocytes (CD11b ${ }^{\text {high }}$

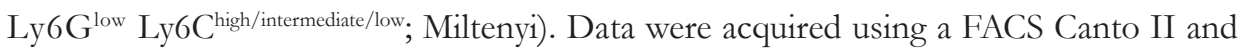
analyzed with FACSdiva software (BD), as described earlier (4).

\section{PLASMA NITRATE AND NITRITE}

Plasma nitrate and nitrite levels in mice receiving drinking water on chow $(n=10)$ served as control. Additionally, plasma nitrate and nitrite levels were measured for mice after 14 weeks of $\mathrm{HCD}$ and $\mathrm{NaNO}_{3}$ or $\mathrm{NaCl}\left(\mathrm{NaNO}_{3}, \mathrm{n}=15 ; \mathrm{NaCl}, \mathrm{n}=19\right)$. Aliquots were stored at $-80^{\circ} \mathrm{C}$ to avoid repeated freezing and thawing. Plasma nitrate $\left(\mathrm{NO}_{3}^{-}\right)$and nitrite $\left(\mathrm{NO}_{2}^{-}\right)$ were determined by a sensitive and selective HPLC system analysis (ENO-20 Eicom Japan). This system uses reverse phase chromatography to separate nitrite from nitrate 
and nitrate is then reduced to nitrite via a cadmium and reduced copper reaction inside a reduction column. The resulting reduced nitrite is subsequently derivatized using Griess reagent and the level of diazo compounds is measured at $540 \mathrm{~nm}$ (36).

\section{WhOLE BLOOD hEMOgLOBIN-BOUND hbNO USING ELECTRON PARAMagNETIC RESONANCE (EPR)}

Changes in levels of nitric oxide in whole blood was determined using electromagnetic spin resonance (ESR) spectroscopy, by detection of the nitrosyl-hemoglobin (NO-Hb) signal using the settings described before (37). In short, ESR spectra were recorded on $1 \mathrm{ml}$ of snap frozen whole blood at $77 \mathrm{~K}$ in a glass finger dewar on a Bruker EMX 1273 spectrometer with an ER 4119HS cavity and $12 \mathrm{~kW}$ power supply, operating at X band frequencies. ESR conditions were as follows: The magnetic field was $3260 \mathrm{G}$ with a sweep width of $200 \mathrm{G}$. The modulation frequency was $100 \mathrm{kHz}$ with a $5 \mathrm{G}$ amplitude, and 10 scans were made. NO concentrations were calculated from the height of three line NO-Hb spectrum with Bruker WINEPR software as described (37).

\section{PLASMA NITROTYROSINE AND AMINO ACID PROFILE USING HPLC}

Plasma samples were obtained from nitrate and $\mathrm{NaCl}$ drinking mice after 14 weeks of $\mathrm{HCD}$. Samples were snap-frozen and aliquots stored at $-80^{\circ} \mathrm{C}$ until further use. Plasma nitration was analyzed using a 3-nitrotyrosine ELISA on plasma samples (OxiSelect; STA-305, Cell Biolabs). For amino acid profiling, 100ul of plasma was deproteinized using $6 \mathrm{mg}$ of 5 -sulfosalicyic acid, vortex-mixed vigorously and stored at $-80^{\circ} \mathrm{C}$ until further use. Prior to analysis, samples were thawed and centrifuged $10 \mathrm{~min}$ at $50.000 \mathrm{xg}$ in a pre-chilled $\left(4^{\circ} \mathrm{C}\right)$ centrifuge. A $5 \mu$ aliquot of the clear supernatant was automatically derivatized with o-phthaldialdehyde and chromatographed as described earlier (38).

\section{STATISTICAL ANALYSIS}

All data are presented as mean \pm SEM. All parameters were analyzed using independent sample tests and were tested for normal distribution using Shapiro-Wilk normality test. Parameters with two groups were compared with student's t-test or Mann-Whitney rank-sum test. In case of more than two groups, parameters were analyzed using 1way ANOVA followed by Bonferroni's Multiple Comparison Test or Kruskal-Wallis ranksum test, followed by Dunn's post-hoc testing. Correlations of plasma nitrate and nitrite levels with time of last nitrate consumption were tested using Pearson or Spearman correlation (GraphPad Prism4). 


\section{RESULTS}

\section{dietary nitrate gupplementation eleVates plasma nitrate LEVELS}

First the effect of a high cholesterol diet (HCD) and equimolar $\mathrm{NaCl}$ (33) on plasma nitrate levels was measured. Plasma nitrate concentrations were not affected by HCD with $\mathrm{NaCl}$ drinking compared to normal drinking water plus a chow diet (Fig. 1A).

Dietary nitrate at a dose of $1 \mathrm{~g} / \mathrm{L}$ is the most common dose described to increase plasma nitrate levels, and corresponds to a physiological nitrate intake achieved by a vegetablerich diet in humans (33). Also in our hands, 14 weeks of dietary nitrate drinking increased plasma nitrate levels compared to sodium drinking controls (Fig. 1B), similarly to levels described (33). No differences were observed in body weight gain and liquid consumption suggesting comparable eating and drinking behavior between groups (Fig. $1 \mathrm{C}, \mathrm{D})$.

Figure 1
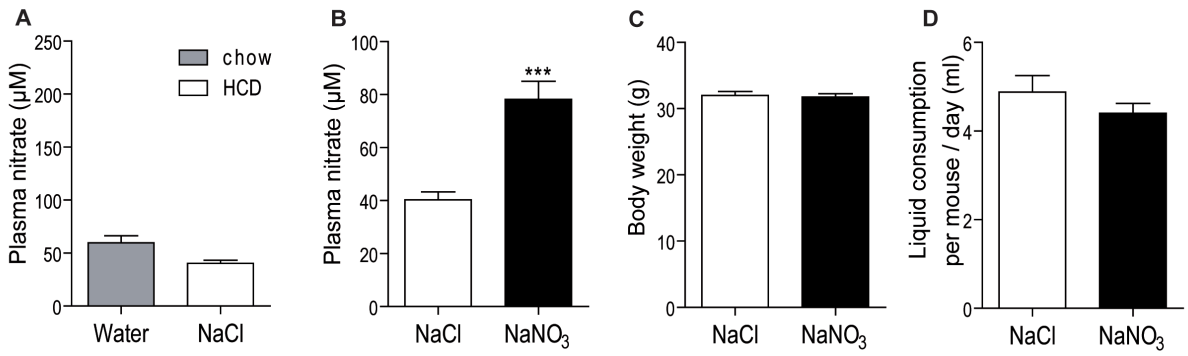

Figure 1: Dietary nitrate syoolementation elevates plasma nitrate levels.

A. Plasma nitrate measured in LDLr/- mice drinking $\mathrm{NaCl}$ on $\mathrm{HCD}$ for 14 weeks as compared to mice with normal drinking water on chow diet. B. Plasma nitrate levels in $\mathrm{HCD}$-fed $\mathrm{LDL} \mathrm{r}^{\circ}$ - mice after drinking $1 \mathrm{~g} / \mathrm{L} \mathrm{NaNO}_{3}$ (black bars) or equimolar $\mathrm{NaCl}$ (white bars) for 14 weeks. ***p-value $<0.0001 \mathrm{NaNO}_{3}$ vs. $\mathrm{NaCl}$ supplementation, student's t-test. C. Body weight and D. average liquid consumption per mouse per day in HCD-fed LDLr/- mice treated with $\mathrm{NaCl}$ or $\mathrm{NaNO}_{3}$ for 14 weeks. Graphs represent mean \pm SEM for 15 mice per group. 


\section{PROLONGED dietary NItRAtE dOES NOT CAUSE gYSTEMIC SIDE-EFFECTS}

Dietary nitrate supplementation has been linked with tumorigenesis. We therefore investigated the systemic effects of prolonged nitrate ingestion.

Morphologically, we did not observe organ tumor development, as analyzed by a certified veterinary pathologist. Nitrates have also recently been suggested to affect platelets and thrombosis (39). However, neither total red blood cell count nor platelet count (Fig. 2A) was affected by dietary nitrate supplementation. Additionally, red blood cell composition, as measured in red blood cell mean cell volume (MCV), mean corpuscular hemoglobin $(\mathrm{MCH})$ and mean corpuscular hemoglobin concentration $(\mathrm{MCHC})$ and hematocrit, did not differ between nitrate and $\mathrm{NaCl}$ treatments (Fig. 2B-E). Together, these data suggest that neither leucocyte count nor erythropoiesis were affected following prolonged dietary nitrate supplementation.

\section{Figure 2}

A

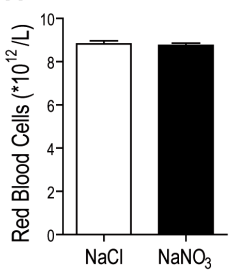

C

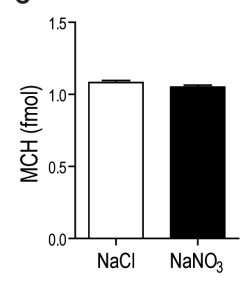

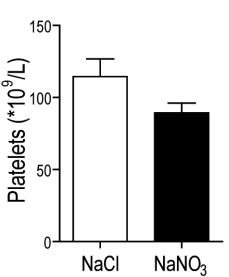

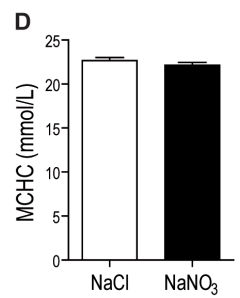

B

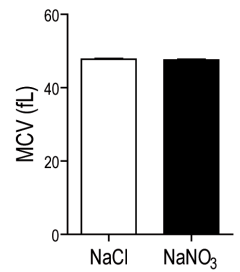

E

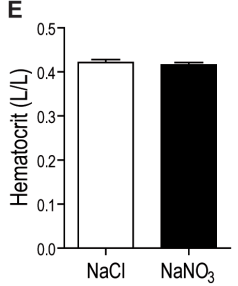

Figure 2: Prolonged dietary nitrate does not cause systemic side effects.

A. Red blood cell content and platelet count after 14 weeles of nitrate supplementation compared to control. B. Coulter counter analysis of mean cell volume (MCV), C. mean corpuscular hemoglobin (MCH), D. mean corpuscular hemoglobin concentration $(\mathrm{MCHC})$ and $\mathrm{E}$. hematocrit level in nitrate and $\mathrm{NaCl}$ drinking mice after 14 weeks of $\mathrm{HCD}$. All data was analyzed using student's t-test.

Additionally, dietary nitrate and $\mathrm{NO}$ have been shown to reduce circulating and adhering leukocytes $(17,22,23,40,41)$. Therefore, we compared innate and adaptive immune cells in blood, spleen and lymph nodes between nitrate supplemented mice and mice receiving the control treatment. However, dietary nitrate supplementation did not modulate circulating white blood cell count (Fig. 3A) or differentiation and distribution 
of inflammatory cells in blood or lymphoid organs (blood: Fig. 3B-H, Supplementary Figure 1, spleen and lymph nodes: data not shown), suggesting that prolonged dietary nitrate supplementation does not lower circulating or tissue-resident immune cells.

Figure 3

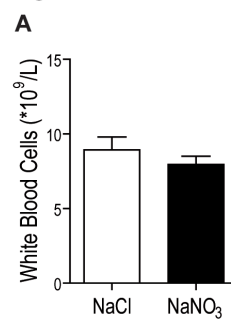

B
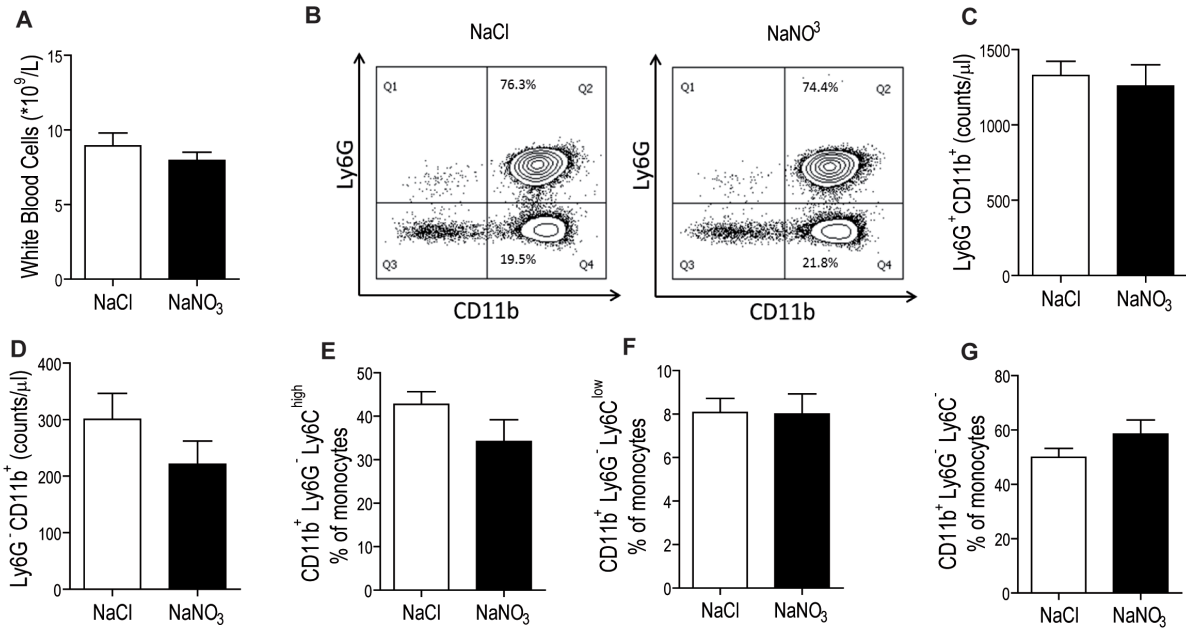

$\mathbf{F}$

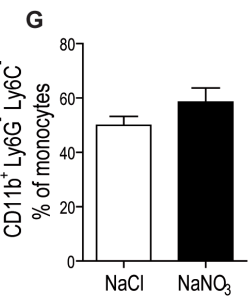

$\mathrm{H}$

\begin{tabular}{|llll|}
\hline Cell type & $\mathrm{NaCl}($ mean \pm SEM) & Nitrate (mean \pm SEM) & p value \\
\hline CD45 $^{+}$leucocytes & $3124 \pm 280.9$ & $2627 \pm 253.3$ & 0.2142 \\
\hline T cells & $362.5 \pm 32.30$ & $321.6 \pm 24.7$ & 0.3453 \\
\hline CD4 $^{+}$T cells & $210.1 \pm 18.24$ & $186.5 \pm 14.46$ & 0.3619 \\
\hline CD8 $^{+}$T cells & $128.0 \pm 12.38$ & $112.9 \pm 9.046$ & 0.3563 \\
\hline B220 $^{+}$B cells & $747.7 \pm 104.1$ & $513.4 \pm 56.52$ & 0.0936 \\
\hline Granulocytes & $1329 \pm 94.64$ & $1258 \pm 141.8$ & 0.6778 \\
\hline
\end{tabular}

Figure 3: Prolonged dietary nitrate does not cause inflammatory side effects.

A. White blood cell count (Coulter counter) in HCD-fed LDLr/- treated with $\mathrm{NaCl}$ or $\mathrm{NaNO}_{3}$ for 14 weeks. B. FACS plot example for granulocyte and monocyte gating in $\mathrm{NaCl}$ and $\mathrm{NaNO}_{3}$ treated mice (see supplement for gating strategy). C. Absolute cell counts acquired by flow cytometry of granulocyte and D. monocyte content. E. Importantly for atherosclerosis, relative contribution of monocyte subsets with Ly6C Chigh F. Ly6C Con and G. Ly6C Cog monocyte subsets. H. Overview of remaining leucocyte subsets, which were all unchanged. Flow cytometry analysis was performed on whole blood of HCD-fed LDLr/ mice treated with $\mathrm{NaCl}$ or $\mathrm{NaNO}_{3}$ for 14 weeks. $\mathrm{NaCl}$ group is presented with white bars and $\mathrm{NaNO}_{3}$ group in black bars. Graphs represent mean \pm SEM for 15 mice per group. All data was analyzed using student's t-test. 
prolonged dietary nitrate supplementation does not afFect atherosclerogls DEVELOPMENT, hyPOXIA OR BLOOD PREgSURE

Next, the effect of prolonged dietary nitrate supplementation on atherosclerosis was studied. Short term dietary nitrate has been shown to prevent microvascular inflammation in hypercholesterolemic C57BL/6J mice, where it also reversed endothelial dysfunction (42). However, $\mathrm{LDLr}^{-/}$mice receiving nitrate supplementation showed similar plaque size and necrotic core development in the aortic root as control (Fig. 4A), while plasma cholesterol and triglyceride (TG) levels were unchanged (Fig. $5 \mathrm{~A}, \mathrm{~B})$. In line with this, advanced plaques in a second location in the arterial tree, the aortic arch (inner curvature and main branch points: brachiocephalic trunk, left carotid artery, subclavian artery), also presented with equal plaque size and necrotic core in $\mathrm{NaNO}_{3}$ and $\mathrm{NaCl}$ supplemented mice, as analyzed as the sum of all plaques in this area (Fig. 4A) and as individual plaques in separate branch points (data not shown). Additionally, macrophage content was equivalent between the nitrate and control group for both the aortic root and arch (Fig. 4B). Also, plaque collagen and smooth muscle cell content did not change upon prolonged dietary nitrate supplementation (Fig. 4C, D). This, together with unchanged cap thickness in both groups (Fig. 4C) suggests no difference in plaque stability upon dietary nitrate supplementation. In addition, blinded semi-quantitative analysis of plaque phenotype (plaque fibrosis, necrosis, granulocyte and adventitial immune cells) by a veterinary pathologist did not reveal any changes (data not shown). In fact, classification of the aortic root plaque stage, as described earlier (43), by a certified veterinary pathologist confirmed similar plaque progression (Fig. 4F). In short, plaques were classified in early lesions with fatty streaks containing only macrophage derived foam-cells, intermediated lesions characterized by the additional presence of a collagenous cap and advanced lesions with involvement of the media and increased collagen content. Prolonged dietary nitrate supplementation hence did not alter plaque progression or phenotype and did not affect macrophage tissue infiltration. 
Figure 4
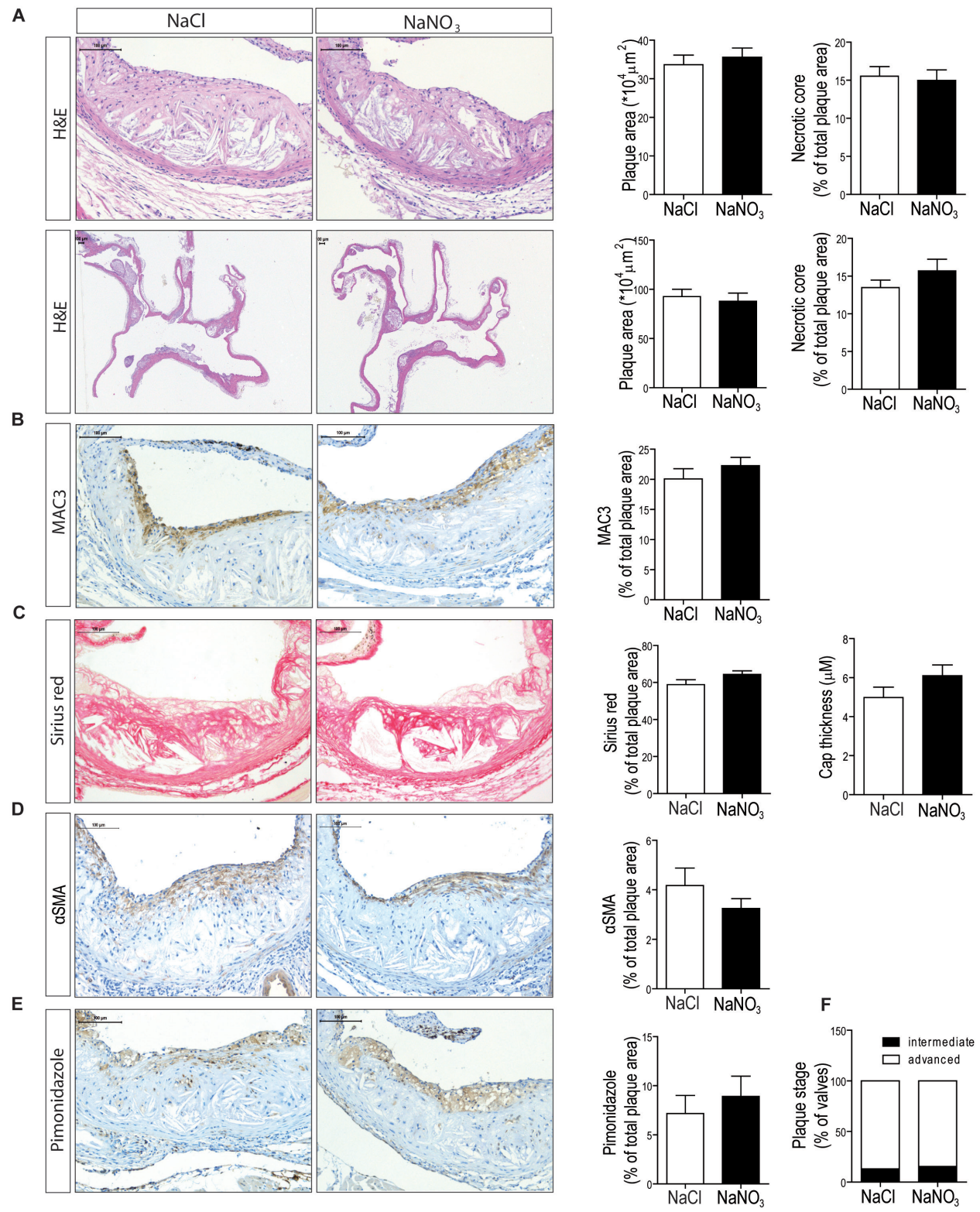

Figure 4: Prolonged dietary nitrate supplementation does not affect atherosclerosis development, hypoxia or blood pressure.

A. Representative pictures and quantifications of H\&Es (bematoxylin \& eosin) of aortic roots and arches, as well as B. MAC3 and staging of plaques of HCD-fed LDLr/- mice treated with $\mathrm{NaCl}$ or $\mathrm{NaNO}_{3}$ for 14 weeks. C. Sirius red quantification as well as cap thickness analysis, D. a-smooth muscle actin (aSMA), and E. Pimonidazole staining 
in aortic arches of $\mathrm{HCD}$-fed $\mathrm{LDL} \mathrm{r}^{/-}$mice treated with $\mathrm{NaCl}$ or $\mathrm{NaNO}_{3}$ for 14 weeks. F. Plaque stage scoring by a certified veterinary pathologist. White bars represent $\mathrm{NaCl}$ and black bars $\mathrm{NaNO}_{3}$ treated mice. Graphs represent mean $\pm S E M$ for 15 mice per group and all data was analyzed using student's t-test.

Additionally, we hypothesized that enhanced mitochondrial function could reverse plaque hypoxia thereby alleviating plaque burden. As shown previously (4), advanced murine plaques were hypoxic, as measured using the hypoxia-specific marker pimonidazole. However, prolonged dietary nitrate did not affect plaque hypoxia in the aortic root of mice having received either 14 weeks of nitrate or control on HCD (Fig. 4E). Oxygen sensitivity and specificity of pimonidazole-mediated detection of tissue hypoxia have been described elsewhere (4).

Moreover, we did not observe changes in blood pressure of long term supplemented mice, as suggested by the literature. Neither mean blood pressure, nor systolic or diastolic blood pressure was affected by prolonged dietary nitrate, as measured by tailcuff plethysmography and catheter probe in the carotid artery (Fig. 5C-E). Also heart rate remained stable between groups (Fig. 5F), suggesting no hemodynamic effects of prolonged dietary nitrate supplementation. In conclusion, prolonged dietary supplementation of nitrate does not alter atherosclerosis development, tissue oxygenation or blood pressure.

\section{Figure 5}
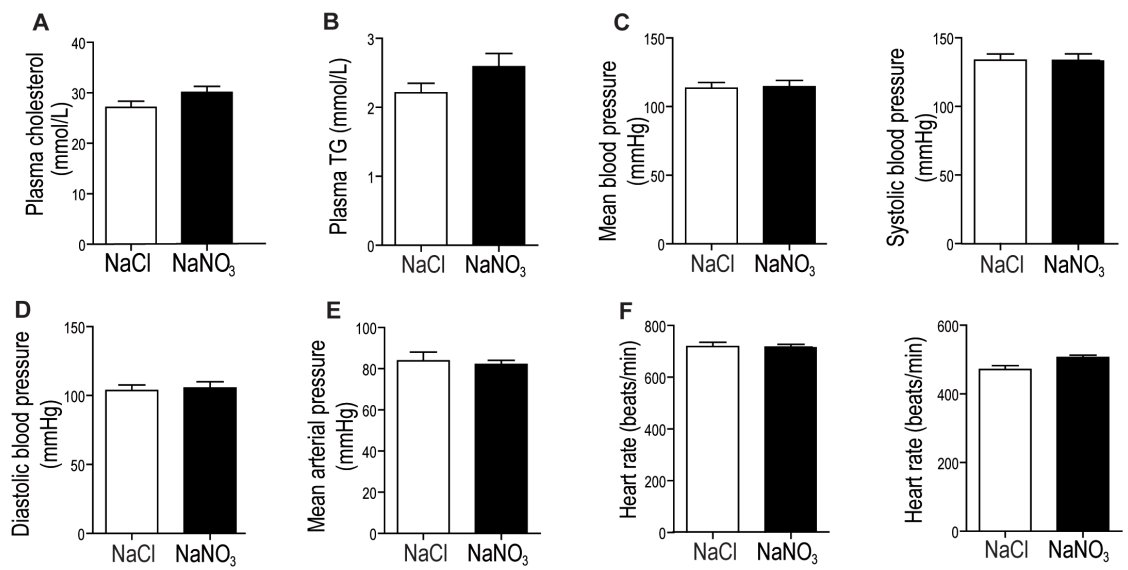

Figure 5: Plasma lipid levels and hemodynamics are unaffected by prolonged dietary nitrate supplementation.

A. Plasma cholesterol and B. plasma triglyceride (TG) levels in $\mathrm{HCD}$-fed LDLr/- mice treated with $\mathrm{NaCl}$ or $\mathrm{NaNO}_{3}$ for 14 weeks. C. Mean blood pressure (left panel), systolic (middle panel) and D. diastolic blood pressure, as measured by tailcuff plethysmography in $\mathrm{HCD}$-fed LDLr/- mice treated with $\mathrm{NaCl}$ or $\mathrm{NaNO}_{3}$ for 14 weeks. E. Mean arterial pressure, measured with a catheter probe in the carotid artery in $\mathrm{NaNO}_{3}$ compared to $\mathrm{NaCl}$ receiving mice after 14 
weeks of HCD. F. Heart rate measured by tail-cuff method (left panel) and catheter probe (right panel) in the thoracic aorta in $\mathrm{HCD}$-fed $\mathrm{LDLrR} /$ mice treated with $\mathrm{NaCl}$ or $\mathrm{NaNO}_{3}$ for 14 weeks. White bars represent $\mathrm{NaCl}$ and black bars $\mathrm{NaNO}_{3}$ treated mice. Graphs represent mean \pm SEM for 15 mice per group and all data was analyzed using student's t-test.

\section{prolonged dietary Nitrate drinking doEs NOt gustain an INCREased NItrate-NItrite-NO PATHWAY}

In order to understand the lack of effects seen, plasma nitrate and nitrite levels were studied over time during dietary nitrate supplementation. Similarly to plasma nitrate, plasma nitrite levels were not affected by $\mathrm{HCD}$ consumption and $\mathrm{NaCl}$ itself (Fig. 6A). Two weeks dietary nitrate supplementation significantly increased plasma nitrite levels (Fig. 6B). However, over time and already after 6 weeks, plasma nitrite levels decrease gradually back to $\mathrm{NaCl}$ control levels after 14 weeks of nitrate administration (Fig. 6B). Also, plasma nitrate levels decrease similarly, but remain significantly elevated at 14 weeks (Fig.6C). Hemoglobin-bound nitric oxide levels were not elevated upon 14 weeks of dietary nitrate supplementation (Fig. 6D, example of electron paramagnetic resonance (EPR) spectrum in Fig. 6E). These data suggest a negative feedback response to prolonged dietary nitrate supplementation. Additionally, there was no correlation between plasma nitrate and nitrite levels with the time point of last nitrate consumption. Three hours after the last nitrate consumption, mice show equivalent plasma nitrate levels compared to mice sacrificed at earlier time points (Fig. 6F).

Mechanistically, compensation at the level of nitrite and NO levels can be mediated by reduced nitrite and NO production by bodily cells themselves or enhanced clearance of nitrate/nitrite from the body. NO fate and production was studied in mice receiving 14 weeks of dietary nitrate supplementation as follows: We studied the citrulline/arginine ratio as a marker of NOS-mediated conversion of arginine into citrulline and simultaneous NO production. Additionally, the ratio of plasma ornithine/citrulline was used as a quantitative indicator of arginine consumption by arginase (44). Together these ratios provide an insight into NOS activity as compared to arginase activity (see Fig. 6G). Both ratios were unaffected (Fig. 6H-I), suggesting that NO production and fate are not altered in mice receiving dietary nitrate. Furthermore, the level of nitrotyrosine in plasma was determined to estimate the conversion of nitrite to peroxynitrite and other reactive nitrogen intermediates (RNI) (45). Plasma nitrotyrosine levels were not affected after 14 weeks of dietary nitrate (Fig. 6J) suggesting on the one hand that compensation also does not take place at this level. On the other hand, plasma tyrosine levels serve as a marker for liver toxicity, which was also not affected by prolonged dietary nitrate consumption. Overall, a slower compensation of plasma nitrate as compared to plasma 
nitrite levels, might suggest initial compensation at the plasma nitrite as opposed to the plasma nitrate level (Fig6 B-C).

Figure 6

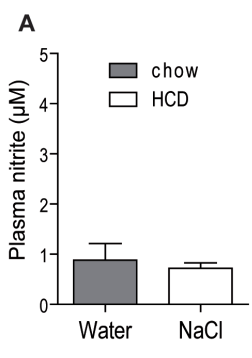

E

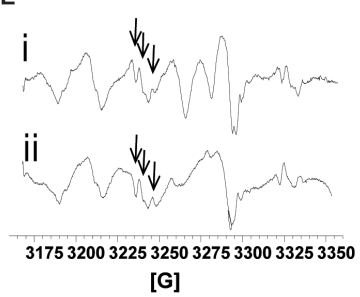

G

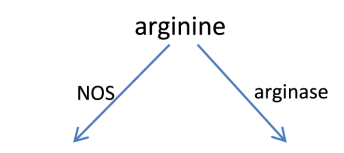

citrulline $\longleftarrow$ ornithine

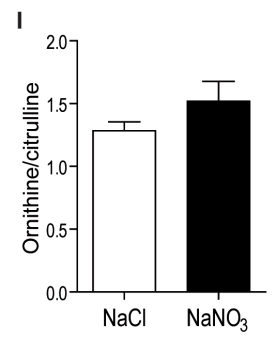

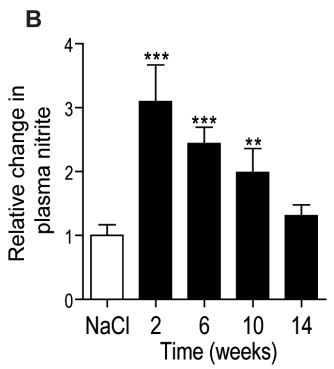
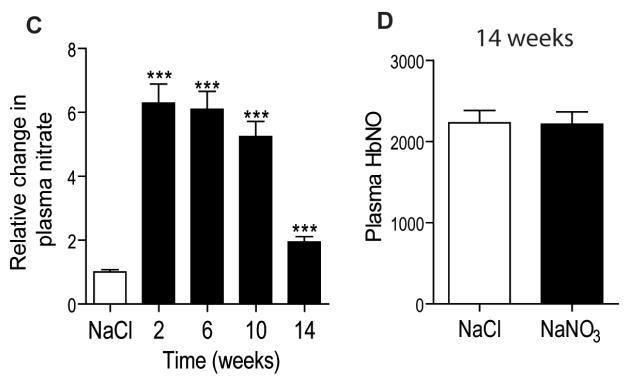

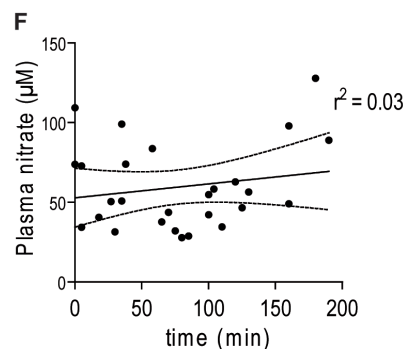

$\mathrm{H}$
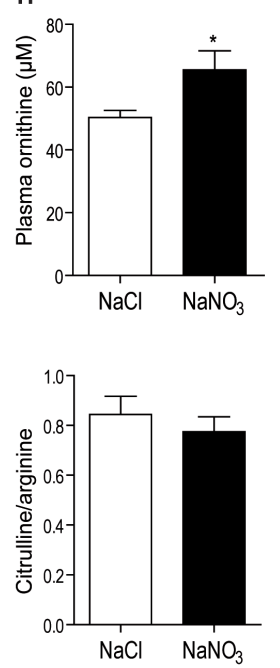
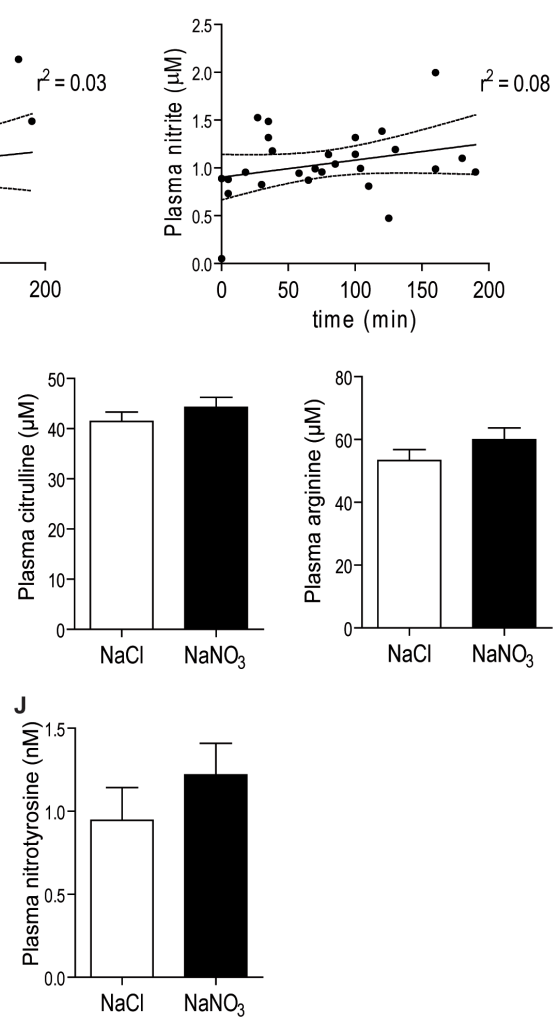

Figure 6: Compensation of prolonged nitrate drinking does not involve eNOS activity and NO scavenging.

A. Plasma nitrite after 14 weeks of $\mathrm{NaCl}$ drinking and $\mathrm{HCD}$ intervention compared to normal water and chow diet exposed mice. B. Relative plasma nitrite and C. nitrate levels after 2, 6, 10 and 14 weeks of dietary nitrate supplementation. Data is presented relative to control $\mathrm{NaCl}$ drinking mice of the same time point. 
D. Hemoglobin-bound nitric oxide levels in $\mathrm{NaNO}_{3}$ compared to $\mathrm{NaCl}$ supplemented mice after 14 weeks of supplementation and HCD. E. Example of an Electron Parametric Resonance (EPR) spectrum showing Hb-NO detected in mouse blood of i) control mouse and ii) nitrate-exposed mouse. Arrows indicate a small three line patterns with a g-factor of 2.05, representing HB-NO. F. Plasma nitrate (left panel) and nitrite (right panel) showed no correlation with the time point of last nitrate consumption (Pearson correlation, GraphPad Prism4). G. Scheme of arginine metabolism. H. Plasma ornithine, citrulline and arginine levels (left, middle and right panel, respectively) as well as I. calculated ornithine/ citrulline and citrulline/ arginine ratios (left and right panel, respectively). J. Plasma nitrotyrosine levels to asses $\mathrm{NO}$ scavenging in mice drinking $\mathrm{NaNO}_{3}$ compared to $\mathrm{NaCl}$ for 14 weeks. Graphs represent mean \pm SEM for 15 mice per group. ***p-value $<0.0001 \mathrm{NaNO}_{3}$ vs. $\mathrm{NaCl}$ supplementation of same time point, student's t-test.

\section{DISCUSSION}

In this study, we show that short term dietary nitrate supplementation increased plasma nitrate and nitrite levels, while following prolonged dietary nitrate supplementation only plasma nitrate levels remained slightly elevated. Plasma nitrite levels decreased earlier than plasma nitrate levels, potentially hinting towards a compensatory mechanism at the level of plasma nitrite. Overall, fourteen weeks of dietary nitrate supplementation did not affect blood pressure, systemic and tissue immune cells or atherosclerosis development. These data suggest that elevated nitrate and nitrite in the initial 6 weeks of dietary nitrate supplementation did either not exert anti-atherosclerotic effects on initial fatty streak formation, first occurring after 4-6 weeks, or effects are overridden upon subsequent plaque advancement. Also, the slight elevation in plasma nitrate levels remaining after 14 weeks was not sufficient to affect plaque burden.

Short term dietary nitrate supplementation has been studied extensively over the past few years. Effects described include blood pressure lowering as well as antiinflammatory effects after acute or short term administration of dietary nitrate, as studied in inflammatory bowel disease models $(17,46)$. Also, short term dietary nitrate supplementation (max. of 12 days) was shown to reduce erythropoiesis capacity in rats (47). In this study we show that upon long term nitrate supplementation, however, these properties, which were expected to be anti-atherosclerotic, are not observed. Additionally, we do not find any systemic side effects of prolonged dietary nitrate supplementation.

Indeed, the main concern of nitrate supplementation used to be cancer development, in particular stomach cancer, due to a reaction of nitrite with dietary amines resulting in carcinogenic nitrosamines. This has led to adjustments in policies of nitrate supplementation in water and processed meat. More recent evidence points at the many health benefits of nitrate supplementation $(32,48,49)$, thereby changing the 
classic view on dietary nitrate supplementation. In our model of prolonged dietary nitrate administration, we did not observe organ tumor development. The latter is not surprising as carcinogenesis with the modest level of nitrate supplementation in the present study is unlikely. A dose of $1 \mathrm{~g} / \mathrm{L} \mathrm{NaNO}_{3}$ equals ca. $150 \mu$ mol nitrate per day based on observed drinking behavior, similar to a vegetable rich diet in humans (33).

Dietary nitrate supplementation has entered clinical trials in cardiovascular disease. However, the results are variable and few long term studies have been performed so far in humans (26-28). While using similar nitrate concentrations as used in our study, beneficial effects on oxygen cost during exercise, cardiovascular function, blood pressure and inflammation have been described upon short term interventions $(8-11,13,15)$. In fact, nitrate supplementation periods of up to 4 weeks for humans and wild type mice elevated both plasma nitrate and nitrite levels on the short term $(27,29)$. In comparison, our data suggests a compensatory negative feedback mechanism upon prolonged dietary nitrate supplementation leading to normalization of plasma nitrite and NO levels and eventually also plasma nitrate levels, over-riding the effects observed following acute or short term dietary nitrate supplementation. In accordance, Carlström et al. have previously described a return to baseline plasma and tissue nitrite levels after 8-10 weeks of prolonged nitrate drinking in mice and rats $(25,50)$.

Also, recently Hezel et al. could confirm a lack of plasma nitrite and nitrate elevation upon 17 month of dietary nitrate supplementation (51). Our results confirm this finding and extend on the compensatory mechanism. Carlström et al. attribute the compensation seen to a feedback down-regulation of eNOS activity in rodents (50), as in addition to $\mathrm{NO}$ generation from nitrate-nitrite, eNOS generates NO from L-arginine. However, compensation was also seen in eNOS deficient mice and reduced NO production might therefore not present the only mechanism (25). In fact, in our hands eNOS activity was probably not affected in mice as suggested by the unaffected ratio of citrulline/ arginine upon prolonged dietary nitrate interventions, confirming other compensatory mechanisms. Alternatively, enhanced clearance of nitrate and nitrite via urine could explain the observed normalization in plasma nitrate and nitrite concentrations after prolonged nitrate supplementation. Sixty to seventy percent of the ingested dietary nitrate is secreted in the urine as studied in rats and humans upon single high dose nitrate supplementation (52,53). Also, 24-hour nitrate was enhanced in subjects with elevated nitrate concentrations in drinking water, compared to subjects drinking water with lower nitrate contamination (54). In fact, already after 7 days of dietary nitrate supplementation increased urinary nitrate excretion by sevenfold (55). Interestingly, Hezel et al. have investigated this upon $17 \mathrm{months}$ of dietary nitrate supplementation and 
found inconclusive data due to an extreme outlier in their data (51). From the available data we suggest that reduced nitrite could hence result from enhanced nitrate or nitrite urinary excretion. As we observed that plasma nitrite reduction preceded plasma nitrate levels, we suggest the compensatory mechanism on the level of plasma nitrite and possibly NO.

Additional explanations for nitrite compensation include reduced nitrite generation from nitrate or enhanced conversion of nitrite into $\mathrm{NO}$ and $\mathrm{NO}$-derived metabolites. The latter is unlikely, as we did not observe changes in plasma nitrotyrosine levels, a biomarker for peroxynitrite generation and other RNI formations (45). These results confirm the previously reported observation that nitrate did not increase nitration of skeletal muscle proteins (9). Additionally, we did not observe any harmful systemic side effects of nitrate supplementation, such as atherosclerosis or plaque necrosis, which would be expected when production of pro-atherogenic RNI is increased (56,57). This also suggests that the prolonged dietary nitrate intervention is safe with respect to RNIinduced disorders. Reduced nitrite generation from nitrate, however, might still present an additional compensatory mechanism. In mammals, nitrate is reduced to nitrite by commensal bacteria only, as mammals lack nitrate reductases themselves. HendgenCotta et al. did indeed show a reduction in plasma nitrite levels in mice which received anti-septic mouth wash to reduce commensal bacterial growth in addition to nitrate supplementation, compared to mice with sole dietary nitrate supplementation (33). Also in men, Bondonno et al could recently show that anti-bacterial mouth wash reduces oral nitrate reduction (58), suggesting that changes in the commensal flora can affect nitrite and subsequent NO availability also in mice and men. However, if commensal bacterial growth and productivity contributes to the compensation seen upon prolonged nitrate drinking in mice, remains to be established. Additionally, plasma nitrite can be derived from ceruloplasmin-mediated oxidation or auto-oxidation of $\mathrm{NO}$ to nitrite (reviewed in (19)). Nevertheless, similar hemoglobin-bound NO levels do not support reduced autooxidation as an alternative compensatory mechanism.

Overall, long term dietary nitrate supplementation does not show beneficial effects on atherosclerosis development in mice. If prolonged dietary nitrate is also compensated for in humans remains to be shown. Alternatively, intermittent therapy might present a novel strategy and provide insights into the compensatory mechanism. In fact, Carlström et al. show a reversible downregulation of eNOS activity upon long term dietary nitrate (50). However, a therapeutic effect of intermittent nitrate drinking remains to be established, but could prove successful in humans, based on the promising effects seen of short term dietary nitrate supplementation. 


\section{CONCLUSION}

There is still debate on potential beneficial and detrimental effects of nitrate supplementation. In this manuscript, we show that prolonged dietary nitrate supplementation abolished beneficial effects of nitrates, while at the same time not causing any systemic side-effects in mice. Short term supplementation of dietary nitrates might hence have therapeutic value, whereas chronic diseases, such as atherosclerosis might not benefit from continuous dietary nitrate supplementation. 


\section{FUNDING}

VENI fellowship of the Dutch Organization for Scientific Research (to J.C.S. 016.116.017); PhD-student Fellowship from the Cardiovascular Research Institute Maastricht (to T.L.T.) 


\section{REFERENCES}

1. WHO. Cardiovascular disease risk [Internet]. WHO.int. 2013. Available from: http://www. who.int/cardiovascular_diseases/en/

2. Tabas I. Macrophage death and defective inflammation resolution in atherosclerosis. Nat Rev Immunol. 2009/12/05 ed. 2010;10(1):36-46.

3. Sluimer JC, Gasc J-M, van Wanroij JL, Kisters N, Groeneweg M, Sollewijn Gelpke MD, et al. Hypoxia, hypoxia-inducible transcription factor, and macrophages in human atherosclerotic plaques are correlated with intraplaque angiogenesis. J Am Coll Cardiol. 2008 Apr 1;51(13):1258-65.

4. Marsch E, Theelen TL, Demandt JAF, Jeurissen M, van Gink M, Verjans R, et al. Reversal of Hypoxia in Murine Atherosclerosis Prevents Necrotic Core Expansion by Enhancing Efferocytosis. Arterioscler Thromb Vasc Biol. 2014 Sep 25;34(12):2545-53.

5. Drager LF, Polotsky VY, Lorenzi-Filho G. Obstructive sleep apnea: an emerging risk factor for atherosclerosis. Chest. 2011 Aug;140(2):534-42.

6. Bradley TD, Floras JS. Obstructive sleep apnoea and its cardiovascular consequences. Lancet. 2009 Jan 3;373(9657):82-93.

7. Drager LF, Bortolotto LA, Figueiredo AC, Krieger EM, Lorenzi GF. Effects of continuous positive airway pressure on early signs of atherosclerosis in obstructive sleep apnea. Am J Respir Crit Care Med. 2007 Oct 1;176(7):706-12.

8. Larsen FJ, Ekblom B, Sahlin K, Lundberg JO, Weitzberg E. Effects of dietary nitrate on blood pressure in healthy volunteers. N Engl J Med. 2006 Dec 28;355(26):2792-3.

9. Larsen FJ, Schiffer TA, Borniquel S, Sahlin K, Ekblom B, Lundberg JO, et al. Dietary inorganic nitrate improves mitochondrial efficiency in humans. Cell Metab. 2011 Feb 2;13(2):149-59.

10. Larsen FJ, Weitzberg E, Lundberg JO, Ekblom B. Effects of dietary nitrate on oxygen cost during exercise. Acta Physiol (Oxf). 2007 Sep;191(1):59-66.

11. Larsen FJ, Weitzberg E, Lundberg JO, Ekblom B. Dietary nitrate reduces maximal oxygen consumption while maintaining work performance in maximal exercise. Free Radic Biol Med. $2010 \mathrm{Jan}$ 15;48(2):342-7.

12. Bryan NS, Calvert JW, Elrod JW, Gundewar S, Ji SY, Lefer DJ. Dietary nitrite supplementation protects against myocardial ischemia-reperfusion injury. Proc Natl Acad Sci U S A. 2007 Nov 27:104(48):19144-9.

13. Bond V, Curry BH, Adams RG, Millis RM, Haddad GE. Cardiorespiratory function associated with dietary nitrate supplementation. Appl Physiol Nutr Metab. 2014 Feb;39(2):168-72.

14. Khalifi S, Rahimipour A, Jeddi S, Ghanbari M, Kazerouni F, Ghasemi A. Dietary nitrate improves glucose tolerance and lipid profile in an animal model of hyperglycemia. Nitric Oxide. 2014 Nov 18;44C:24-30.

15. Breese BC, McNarry MA, Marwood S, Blackwell JR, Bailey SJ, Jones AM. Beetroot juice supplementation speeds $\mathrm{O}_{2}$ uptake kinetics and improves exercise tolerance during severe-intensity exercise initiated from an elevated metabolic rate. Am J Physiol Regul Integr Comp Physiol. 2013 Dec 15;305(12):R1441-50.

16. Berry MJ, Justus NW, Hauser JI, Case AH, Helms CC, Basu S, et al. Dietary nitrate supplementation improves exercise performance and decreases blood pressure in COPD patients. Nitric Oxide. 2015 Aug 1;48:22-30.

17. Jädert C, Petersson J, Massena S, Ahl D, Grapensparr L, Holm L, et al. Decreased leukocyte recruitment by inorganic nitrate and nitrite in microvascular inflammation and NSAID-induced intestinal injury. Free Radic Biol Med. 2012 Feb 1;52(3):683-92.

18. Duncan C, Dougall H, Johnston P, Green S, Brogan R, Leifert C, et al. Chemical generation of nitric oxide in the mouth from the enterosalivary circulation of dietary nitrate. Nat Med. Nature Publishing Group; 1995 Jun;1(6):546-51. 
19. Lundberg JO, Weitzberg E, Gladwin MT. The nitrate-nitrite-nitric oxide pathway in physiology and therapeutics. Nat Rev Drug Discov. 2008 Feb;7(2):156-67.

20. Joannides R, Haefeli WE, Linder L, Richard V, Bakkali EH, Thuillez C, et al. Nitric oxide is responsible for flow-dependent dilatation of human peripheral conduit arteries in vivo. Circulation. 1995 Mar 1;91(5):1314-9.

21. Ignarro LJ. Experimental evidences of nitric oxide-dependent vasodilatory activity of nebivolol, a third-generation beta-blocker. Blood Press Suppl. 2004 Oct;1:2-16.

22. Hossain M, Qadri SM, Liu L. Inhibition of nitric oxide synthesis enhances leukocyte rolling and adhesion in human microvasculature. J Inflamm (Lond). 2012 Jan;9(1):28.

23. Ignarro LJ, Napoli C. Novel features of nitric oxide, endothelial nitric oxide synthase, and atherosclerosis. Curr Diab Rep. 2005 Feb;5(1):17-23.

24. Voetsch B, Jin RC, Loscalzo J. Nitric oxide insufficiency and atherothrombosis. Histochem Cell Biol. 2004 Oct;122(4):353-67.

25. Carlström M, Larsen FJ, Nyström T, Hezel M, Borniquel S, Weitzberg E, et al. Dietary inorganic nitrate reverses features of metabolic syndrome in endothelial nitric oxide synthase-deficient mice. Proc Natl Acad Sci U S A. 2010 Oct 12;107(41):17716-20.

26. Siddiqi N, Neil C, Bruce M, MacLennan G, Cotton S, Papadopoulou S, et al. Intravenous sodium nitrite in acute ST-elevation myocardial infarction: a randomized controlled trial (NIAMI). Eur Heart J. 2014 May 14;35(19):1255-62.

27. Kapil V, Khambata RS, Robertson A, Caulfield MJ, Ahluwalia A. Dietary Nitrate Provides Sustained Blood Pressure Lowering in Hypertensive Patients: A Randomized, Phase 2, Double-Blind, Placebo-Controlled Study. Hypertension. 2014 Nov 24;65(2):320-7.

28. Zamani P, Rawat D, Shiva-Kumar P, Geraci S, Bhuva R, Konda P, et al. Effect of inorganic nitrate on exercise capacity in heart failure with preserved ejection fraction. Circulation. 2015 Jan 27;131(4):371-80.

29. Vanhatalo A, Bailey SJ, Blackwell JR, DiMenna FJ, Pavey TG, Wilkerson DP, et al. Acute and chronic effects of dietary nitrate supplementation on blood pressure and the physiological responses to moderate-intensity and incremental exercise. Am J Physiol Regul Integr Comp Physiol. 2010 Oct;299(4):R1121-31.

30. Lansley KE, Winyard PG, Fulford J, Vanhatalo A, Bailey SJ, Blackwell JR, et al. Dietary nitrate supplementation reduces the $\mathrm{O}_{2}$ cost of walking and running: a placebo-controlled study. J Appl Physiol. 2011 Mar;110(3):591-600.

31. Sandbakk SB, Sandbakk Ø, Peacock O, James P, Welde B, Stokes K, et al. Effects of acute supplementation of L-arginine and nitrate on endurance and sprint performance in elite athletes. Nitric Oxide. 2015 Aug 1;48:10-5.

32. Katan MB. Nitrate in foods: harmful or healthy? Am J Clin Nutr. 2009 Jul;90(1):11-2.

33. Hendgen-Cotta UB, Luedike P, Totzeck M, Kropp M, Schicho A, Stock P, et al. Dietary nitrate supplementation improves revascularization in chronic ischemia. Circulation. 2012 Oct 16;126(16):1983-92.

34. Dutch_Drinking_water_statistics_2012 [Internet]. [cited 2014 Nov 24]. Available from: http://www.vewin.nl/SiteCollectionDocuments/Publicaties/English__publications/Vewin_Dutch_ Drinking_water_statistics_2012.pdf

35. Daugherty A, Rateri D, Hong L, Balakrishnan A. Measuring blood pressure in mice using volume pressure recording, a tail-cuff method. J Vis Exp. 2009 Jan;(27).

36. Jansson EA, Huang L, Malkey R, Govoni M, Nihlén C, Olsson A, et al. A mammalian functional nitrate reductase that regulates nitrite and nitric oxide homeostasis. Nat Chem Biol. 2008 Jul;4(7):411-7. 
37. Wijnands KAP, Vink H, Briedé JJ, van Faassen EE, Lamers WH, Buurman WA, et al. Citrulline a more suitable substrate than arginine to restore $\mathrm{NO}$ production and the microcirculation during endotoxemia. PLoS One. 2012 Jan;7(5):e37439.

38. van Eijk HM, Rooyakkers DR, Deutz NE. Rapid routine determination of amino acids in plasma by high-performance liquid chromatography with a 2-3 microns Spherisorb ODS II column. J Chromatogr. 1993 Oct 22;620(1):143-8.

39. Park JW, Piknova B, Huang PL, Noguchi CT, Schechter AN. Effect of blood nitrite and nitrate levels on murine platelet function. PLoS One. 2013 Jan;8(2):e55699.

40. Kubes P, Suzuki M, Granger DN. Nitric oxide: an endogenous modulator of leukocyte adhesion. Proc Natl Acad Sci. 1991 Jun 1;88(11):4651-5.

41. Cerwinka W, Granger DN. Nitric Oxide Modulates Leukocyte-Endothelial Cell Adhesion. Humana Press; 2000 Jan 1;139-51.

42. Stokes KY, Dugas TR, Tang Y, Garg H, Guidry E, Bryan NS. Dietary nitrite prevents hypercholesterolemic microvascular inflammation and reverses endothelial dysfunction. Am J Physiol Heart Circ Physiol. 2009 May 1;296(5):H1281-8.

43. Xanthoulea S, Thelen M, Pöttgens C, Gijbels MJJ, Lutgens E, de Winther MPJ. Absence of p55 TNF receptor reduces atherosclerosis, but has no major effect on angiotensin II induced aneurysms in LDL receptor deficient mice. PLoS One. 2009 Jan;4(7):e6113.

44. Jung C, Gonon AT, Sjöquist P-O, Lundberg JO, Pernow J. Arginase inhibition mediates cardioprotection during ischaemia-reperfusion. Cardiovasc Res. 2010 Jan 1;85(1):147-54.

45. Herce-Pagliai C, Kotecha S, Shuker DE. Analytical methods for 3-nitrotyrosine as a marker of exposure to reactive nitrogen species: a review. Nitric Oxide. 1998 Jan;2(5):324-36.

46. Jädert C, Phillipson M, Holm L, LundbergJO, Borniquel S. Preventive and therapeutic effects of nitrite supplementation in experimental inflammatory bowel disease. Redox Biol. 2014 Jan;2:73-81. 47. Ashmore T, Fernandez BO, Evans CE, Huang Y, Branco-Price C, Griffin JL, et al. Suppression of erythropoiesis by dietary nitrate. FASEB J. 2015 Mar;29(3):1102-12.

48. Bryan NS, Alexander DD, Coughlin JR, Milkowski AL, Boffetta P. Ingested nitrate and nitrite and stomach cancer risk: an updated review. Food Chem Toxicol. 2012 Oct;50(10):3646-65.

49. Weitzberg E, Lundberg JO. Novel aspects of dietary nitrate and human health. Annu Rev Nutr. 2013 Jan;33:129-59.

50. Carlström M, Liu M, Yang T, Zollbrecht C, Huang L, Peleli M, et al. Cross-talk Between Nitrate-Nitrite-NO and NO Synthase Pathways in Control of Vascular NO Homeostasis. Antioxid Redox Signal. 2015 Aug 1;23(4):295-306.

51. Hezel MP, Liu M, Schiffer TA, Larsen FJ, Checa A, Wheelock CE, et al. Effects of long-term dietary nitrate supplementation in mice. Redox Biol. 2015 May 29;5:234-42.

52. Wagner DA, Young VR, Tannenbaum SR, Schultz DS, Deen WM. Mammalian nitrate biochemistry: metabolism and endogenous synthesis. IARC Sci Publ. 1984 Jan;(57):247-53.

53. Bartholomew B, Hill MJ. The pharmacology of dietary nitrate and the origin of urinary nitrate. Food Chem Toxicol. 1984 Oct;22(10):789-95.

54. van Maanen JM, Welle IJ, Hageman G, Dallinga JW, Mertens PL, Kleinjans JC. Nitrate contamination of drinking water: relationship with HPRT variant frequency in lymphocyte DNA and urinary excretion of N-nitrosamines. Environ Health Perspect. 1996 May;104(5):522-8.

55. Bondonno CP, Liu AH, Croft KD, Ward NC, Puddey IB, Woodman RJ, et al. Short-term effects of a high nitrate diet on nitrate metabolism in healthy individuals. Nutrients. 2015 Jan;7(3):190615.

56. Salisbury D, Bronas U. Reactive oxygen and nitrogen species: impact on endothelial dysfunction. Nurs Res. Jan;64(1):53-66.

57. Virág L, Szabó E, Gergely P, Szabó C. Peroxynitrite-induced cytotoxicity: mechanism and opportunities for intervention. Toxicol Lett. 2003 Apr 11;140-141:113-24. 
58. Bondonno CP, Liu AH, Croft KD, Considine MJ, Puddey IB, Woodman RJ, et al. Antibacterial mouthwash blunts oral nitrate reduction and increases blood pressure in treated hypertensive men and women. Am J Hypertens. 2015 May;28(5):572-5. 


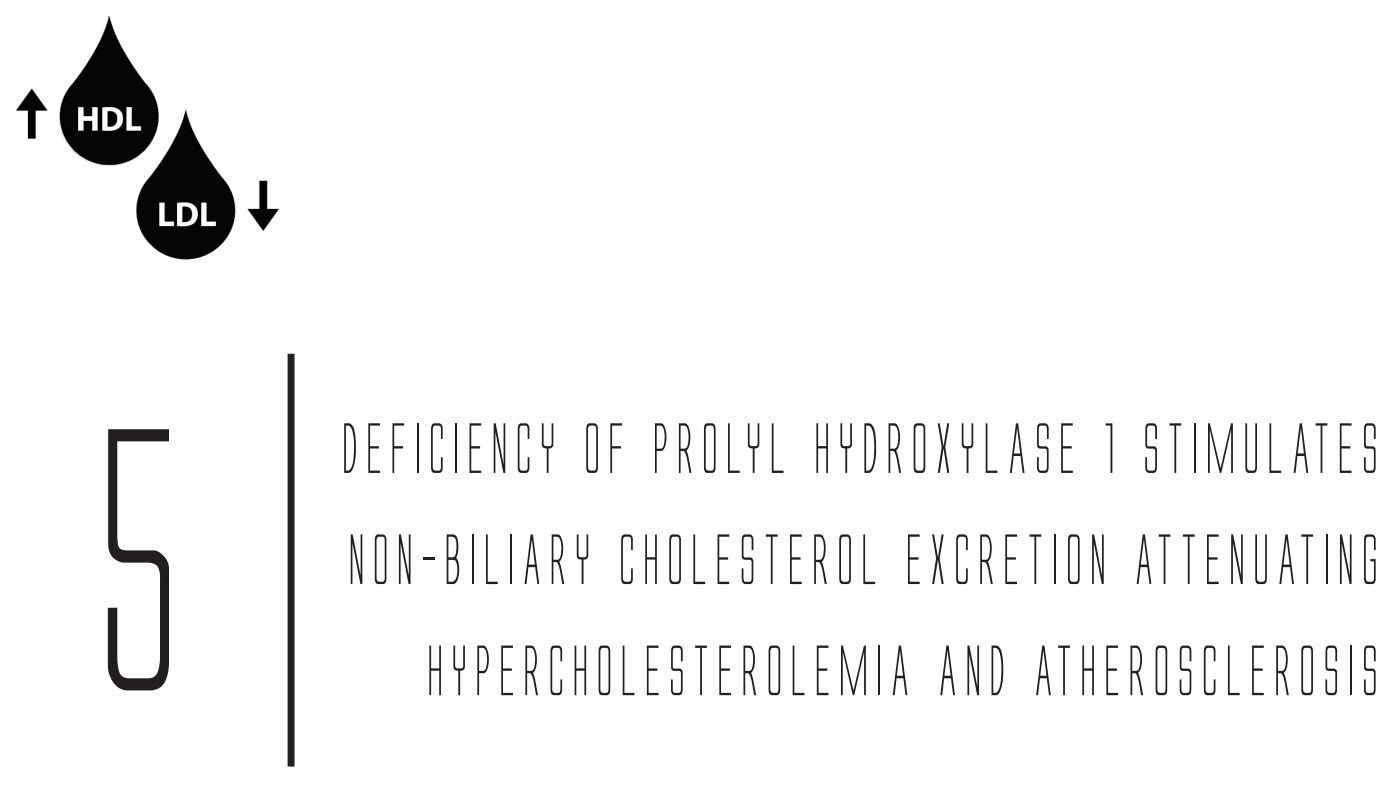

Marsch e, demand Jaf, theelen tl, tullemans bMe, wouters K, boON mr, Van DIJK th, Glubels MJ, OUBOIS LJ, MEEX gJR, MAZZONE M, hUNG G, fISHER EA, BIESSEN EAL, DAEMEN MJAP, RENSEN PCN, CARMELIET P, GROEN AK, SLUIMER JC modifIEd from: EUROPEAN heART JOURNAL, BRIEF COMMUNICATION, ACGEPTED. 



\section{ABSTRACT}

\section{BACKGROUND}

Atherosclerotic plaque hypoxia is present across species and has recently been shown to contribute to atherosclerosis pathogenesis by promoting plaque destabilization. Deficiency of HIF prolyl hydroxylase 1 (PHD1) switches metabolism towards glycolysis and reduces cellular oxygen consumption. We hypothesized that reduced cellular oxygen consumption by PHD1 deficiency alleviates plaque hypoxia and atherogenesis.

\section{APPROACH AND RESULTS}

$\mathrm{LDLr}^{-/}$and $\mathrm{PHD}^{-/} \mathrm{LDLr}^{-/}$mice ( $\mathrm{n}=17$ and 10, respectively) were placed on a diet containing $0.25 \%$ cholesterol (HCD) for 8 weeks and atherosclerosis was analyzed in aortic roots and arches. Plaque hypoxia, detected by pimonidazole injections, and plaque size were indeed reduced by $32 \%$ and $35 \%$, respectively, in $\mathrm{PHD}^{-/} \mathrm{LDLr}^{-/}$mice, independent of plaque macrophage content (MAC3), as main determinant of hypoxia. Interestingly, $\mathrm{PHD}^{-/}{ }^{-\mathrm{LDLr}^{-/}}$mice also exhibited 5-fold reduced plasma cholesterol levels on chow and on HCD, explaining the observed reduction in plaque development. All these effects were independent of erythropoiesis stimulation associated with general PHD inhibition. Bone marrow transplantations did not affect plasma cholesterol or atherosclerosis, suggesting a stromal effect of PHD1. Mechanistically, whole body cholesterol flux analysis showed 4-fold enhanced non-biliary cholesterol excretion directly from plasma into intestines and feces. Simultaneously, hepatic cholesterol uptake, storage and synthesis were not affected in $\mathrm{PHD}^{-/} \mathrm{LDLr}^{-/}$mice, while biliary cholesterol excretion was even slightly decreased. In vivo antisense oligonucleotide-mediated knockdown of hypoxia-inducible factor $2 \alpha$, the main PHD1 target, showed that PHD1 effects were independent of HIF-2 $\alpha$ stabilization.

\section{CONCLUSION}

In conclusion, PHD1 deficiency protects against atherosclerosis by improving non-biliary cholesterol clearance independent of HIF, HMG-CoA-reductase and LDLr. 
INTRODUCTION

Atherosclerotic plaque hypoxia was recently shown to contribute to disease progression, adding another risk factor to the well-characterized risk factors for atherosclerosis development, such as high blood cholesterol, smoking and hypertension (1). Both, human, rabbit and murine atherosclerotic plaques display hypoxic areas (2-4), predominantly located in inflammatory foci. Non-invasive imaging of human plaque hypoxia using $\left[{ }^{18} \mathrm{~F}\right]-\mathrm{HX} 4$ correlated well with $\left[{ }^{18} \mathrm{~F}\right]$-fluorodeoxyglucose uptake, confirming this close association between hypoxia and inflammation (5). Plaque hypoxia is, however, more than an epiphenomenon of inflammation, as reversal of hypoxia using hyperoxic carbogen gas $\left(95 \% \mathrm{O}_{2}, 5 \% \mathrm{CO}_{2}\right)$ was able to block plaque progression and necrosis (4). Here, we aimed to reverse hypoxia using a genetically modified mouse model with reduced oxygen consumption to study the reoxygenation and potential anti-atherosclerotic effects of deficiency in the oxygen sensor prolyl hydroxylase 1 (PHD1, EGLN2) in murine atherosclerosis.

PHD enzymes 1, 2 and 3 are known for their role in hypoxia-inducible factor $\alpha$ (HIF $\alpha$ ) stabilization. In the presence of oxygen, PHDs mediate hydroxylation of two critical prolines of the HIF $\alpha$ proteins, which subsequently targets $\mathrm{HIF} \alpha$ for von Hippel Lindau (vHL) protein-mediated ubiquitination and proteasomal degradation. In hypoxia, hydroxylation capacity of PHDs is inhibited, resulting in $\mathrm{HIF} \alpha$ stabilization (6). The ensuing HIF-mediated transcription limits energy- and oxygen consuming processes, while promoting angiogenesis and thus oxygen supply. The PHD isoforms have differing affinity for the HIF $\alpha$ subunits (7), potentially underlying the differential functions of PHDs. Interestingly, deficiency of PHD1 results in a HIF- $2 \alpha$-mediated metabolic shift from oxidative phosphorylation towards glycolysis, thereby reducing cellular oxygen consumption. This shift was observed to be independent of compensatory fatty acid beta-oxidation or upregulation of PHD2 and PHD3 isoforms (8).

Based on these findings, we hypothesized that the reduced cellular oxygen consumption mediated by PHD1 deficiency can restore plaque oxygen levels, thereby preventing plaque progression in a hypercholesterolemic mouse model. Here, we show that genetic PHD1 deficiency indeed alleviated plaque hypoxia and plaque burden in atherosclerosis-prone low density lipoprotein receptor deficient $\left(\mathrm{LDLr}^{-/}\right)$mice. However, PHD1 deficiency also reduced plasma cholesterol levels. Mechanistically, lower plasma cholesterol levels were due to enhanced non-biliary cholesterol excretion, so called trans-intestinal cholesterol excretion (TICE). Thereby, this study unveils an intriguing new link between oxygen-sensing and cholesterol metabolism. 


\section{MATERIAL AND METHODS}

\section{ANIMALS}

All mouse experiments were approved by the regulatory authority of the Maastricht University Medical Centre and performed in compliance with the Dutch governmental guidelines. PHD1 ${ }^{-/}$mice were generously provided by Prof. Carmeliet (KU Leuven, (8)) and were bred at least 9x on $\mathrm{LDLr}^{-/} \mathrm{C} 57 \mathrm{~J} / \mathrm{Bl6}$ background. Male $\mathrm{LDLr}^{-/}$mice were obtained from an in-house breeding colony, originating from Charles River (Wilmington, MA, USA). Complete absence of PHD1 mRNA was confirmed in bone-marrow derived macrophages, liver and intestines (data not shown).

\section{ATHEROSCLEROSIS MODELS}

Male $\mathrm{PHD}^{-/} \mathrm{LDLr}^{-/}$and LDLr ${ }^{-/}$littermates ( $\mathrm{n}=10$ and 17, respectively, age: 11 weeks) were fed a high cholesterol diet (HCD) ad libitum (0.25\% cholesterol, SDS 824171) for 8 weeks. For bone marrow transplantations, $\mathrm{LDLr}^{-/}$recipients and $\mathrm{PHD}^{-/} \mathrm{LDLr}^{-/-}$ recipients were placed on antibiotic water, containing neomycin $(100 \mathrm{mg} / \mathrm{L}$; Gibco, Carlsbad, CA, USA) and polymyxin B sulfate (60.000U/L; Gibco) for 7 weeks in total while being fed a chow diet. After one week of antibiotic water drinking, bone marrow as isolated from female wild-type (WT; C57J/B16) and PHD1 ${ }^{-/}$mice and transplanted (1x107 cells/mouse) into both lethally irradiated $\mathrm{LDLr}^{-/}$recipients $(2 \mathrm{x} 6 \mathrm{~Gy}, \mathrm{n}=20 / 20)$ and $\mathrm{PHD}^{-/} \mathrm{LDLr}^{-/}$recipients $(\mathrm{n}=8 / 8)$. Mice were left to recover for 6 weeks on chow diet and subsequently placed on HCD for 8 weeks. An additional set of lethally irradiated $\mathrm{LDLr}^{-/}$recipient mice received either WT ( $\mathrm{n}=22$ recipients) or PHD1 ${ }^{-/}$( $\mathrm{n}=15$ recipients) bone marrow. Upon recovery and 4 weeks of HCD, mice underwent bilateral, perivascular collar placement surgery, as previously described (9). These mice were sacrificed after another 13 weeks of HCD (21 weeks post-BMT).

\section{ATHEROSCLEROSIS QUANTIFICATION AND IMMUNOHISTOCHEMISTRY}

One hour prior to sacrifice, all mice were intraperitoneally (i.p.) injected with the hypoxiaspecific marker pimonidazole (100 mg/kg, hypoxyprobe Omni HP3 kit, Hypoxyprobe Inc. Burlington, MA, USA). Mice were euthanized with a pentobarbital overdose (100 mg/kg i.p.) and blood was withdrawn via the right ventricle for flow cytometry, absolute white and red blood cell counts (Coulter Ac.T diff, Beckman Coulter) and total cholesterol analysis. Mice were perfused via the left cardiac ventricle with PBS containing sodium nitroprusside (0.1 mg/ml; Sigma-Aldrich, Seelze, Germany). 
Aortic arch, root and organs were subsequently excised and fixed in 1\% PFA overnight and paraffin-embedded.

Aortic roots and arches were serially sectioned and stained with hematoxylin and eosin (H\&E, Sigma) for plaque area and lipid core content quantification. Five consecutive H\&E sections at $20 \mu \mathrm{m}$ intervals were analyzed blindly using computerized morphometry (Leica QWin V3, Cambridge, UK) and averaged per mouse. Sections within this 100 $\mu \mathrm{m}$ interval were used for remaining immunohistochemical stainings. For all stainings, antigen retrieval was performed at pH 6 (Dako REAL target retrieval, Dako). Hypoxia was detected in the aortic roots and livers using a rabbit polyclonal antibody (clone 2627) directed against pimonidazole derivates, formed specifically in hypoxic but living cells in vivo (\% pimonidazole/viable plaque area). Additionally, macrophage content of aortic root plaques was quantified as $\mathrm{MAC}^{+}$cells/plaque area (rat anti-mouse MAC-3, clone $\mathrm{M} 3 / 84, \mathrm{BD})$ and leucocyte count was quantified in the liver as CD45 positive cells (rat-anti-mouse CD45 LCA ly-5 antibody, clone 30F11 B\&D) per liver area. Oil red $\mathrm{O}$ staining (ORO) on optimum cutting temperature medium (OCT)-frozen tissue allowed quantification of ORO positive area per total liver area as indicator for hepatic fat accumulation.

\section{HUMAN ATHEROSCLEROSIS}

All experiments were conducted in agreement with the code for proper secondary use of human tissue in the Netherlands (http://www.fmwv.nl). PHD1 protein expression was assessed by immunofluorescence in human carotid autopsy samples $(n=38$, mean age 72 years, 64\% men) representing the following stages of atherosclerosis: intimal thickening, pathological intimal thickening, thick fibrous cap (stable) atheroma, and plaque with intraplaque haemorrhage. After antigen retrieval (target retrieval DAKO), slides were incubated overnight with rabbit anti-human PHD1 antibody (1:100 Novus Biologicals NB100-310), followed by biotin-conjugated donkey-anti-rabbit, streptavidinhorseradish peroxidase (hrp) and Cy3 labelled tyramide signal amplification reagent (Perkin Elmer). Nuclei were visualised with DAPI. PHD1 mRNA expression in 21 thick fibrous cap atheromas (stable) and 23 ruptured plaques was analyzed by microarray derived from human carotid endarterectomy, as described in (10). In short, flanking sites of tissue used for transcriptomics analysis were formalin-fixed for subsequent plaque stage analysis. PHD1 mRNA expression intensities from microarrays was correlated with morphometrically analyzed histological plaque characteristics: plaque size, lipid core, necrotic core ( $\%$ of plaque), haemorrhage ( $\%$ of plaque), microvessels $(\%$ CD 31 of plaque), macrophages ( $\%$ CD 68 of plaque), T-cells ( $\%$ CD3 of plaque). Classification of 
all used plaques was performed on hematoxylin and eosin (HE) stained slides according to Virmani et al. (11) by two investigators independently (T.L.T. and J.C.S.).

\section{CHOLEgTEROL FLUX ANALYSIS IN VIVO}

$\mathrm{PHD}^{-/} \mathrm{LDLr}^{-/}$and $\mathrm{LDLr}^{-/-}$mice ( $\mathrm{n}=10 /$ group) were fed a HCD for 8 weeks and subsequently received an intravenous dose of $0.3 \mathrm{mg}(0.763 \mathrm{~mol})$ cholesterol- $\mathrm{D}_{5}$ dissolved in Intralipid (20\%, Fresenius Kabi, Den Bosch, The Netherlands) and an oral dose of $0.6 \mathrm{mg}(1.535 \mathrm{~mol})$ cholesterol-D $\mathrm{D}_{7}$ dissolved in medium chain TG oil. Blood spots were collected from the tail on filter paper before administration of labeled cholesterol and 3, 6, 12, 24, 48, 72, 96, 120, 144 and 168 hours thereafter. At this point, mice received $\left[1-{ }^{13} \mathrm{C}\right]$ acetate (Isotec, Miamisburg, OH) in drinking water $(2 \% \mathrm{w} / \mathrm{w}$ in tap water). Feces (1x daily) and blood spots (0, 8, 24, 32, 48, 56 and 72 hours) were collected from individually housed mice. Food intake and drinking behavior was monitored by weighing food and drinking water during the whole experiment. Seventy-two hours after $\left[1-{ }^{13} \mathrm{C}\right]$ acetate administration, mice were anesthetized by intraperitoneal injection with Hypnorm (fentanyl/fluanisone, $1 \mathrm{ml} / \mathrm{kg}$ ) and Diazepam $(10 \mathrm{mg} / \mathrm{kg}$ ). Bile was collected by cannulation of the gallbladder for $30 \mathrm{~min}$, during which body temperature was stabilized using a humidified incubator. At the end of the experiment, mice were euthanized by exsanguination and livers and intestines were excised.

Analytical procedures are described elsewhere (12). In short, cholesterol was extracted from blood spots (13). Total biliary and plasma concentrations of cholesterol were subsequently determined (14). Biliary lipids (15) and fecal neutral sterols (16) were extracted and derivatized using N,O-bis-(trimethyl)trifluoroacetamide/pyridine (1:1 $\mathrm{v} / \mathrm{v}$ ) with $1 \%$ trimethylchlorosilane at room temperature.

\section{WhOLE bOdy cholesterol FlUX calcUlations}

For cholesterol kinetic analysis, the fractional contributions of the different isotopologues as measured by GCMS ( $\mathrm{m} 0-\mathrm{m} 7)$ were adjusted for the natural abundance of ${ }^{13} \mathrm{C}$ by multi-linear regression as described by Lee et al. (17). This way, the excess fractional distribution of mass isotopologues (M0-M7) due to dilution and incorporation of tracers during the experiment was obtained. In this procedure, the sample taken before the experiment of each animal was used as baseline distribution and so M0 equals one and subsequently, M1 to M7 equals zero for these samples. Empirically, it was found that the time-course of both cholesterol tracers can be described by the sum of three exponential curves: 


$$
f^{t r}(t)=f_{1}^{t r} e^{-k_{1} t}+f_{2}^{t r} e^{-k_{2} t}-f_{3}^{t r} e^{-k_{3} t}
$$

in which $\mathrm{f}$ represents the fractional contribution of the tracer at time point 0 and $\mathrm{k}$ the elimination/absorption rates of the tracer.

To estimate the kinetic parameters in this equation the model was implemented in SAAM II (v2.3, The Epsilon Group, Charlottesville, VA, USA). The area under the curve (AUC) was calculated as the sum of the ratios of the fractional contribution of the tracer ( $\mathrm{fx}$ ) and the fractional elimination/absorption rates $(\mathrm{kx})$ of the three exponential curves.

$$
A U C^{t r}=\frac{f_{1}^{t r}}{f_{1}^{t r}}+\frac{f_{2}^{t r}}{f_{2}^{t r}}-\frac{f_{3}^{t r}}{f_{3}^{t r}}
$$

The turnover rate plasma cholesterol $(\mathrm{Ra})$ is calculated as the amount of the IVadministered cholesterol tracer, i.e., the product of the administered tracer dose (D) and its fractional availability (F) divided by its AUC. The pool size (Q) is calculated as tracer dose of the administered IV cholesterol divided by the intercept with the $\mathrm{y}$-axis (f3). Cholesterol absorption (Rabs) is calculated as the ratio of AUC of OR and IV administered cholesterol that is corrected for its respective dose.

$$
R a=\frac{F^{I V} \times D^{I V}}{A U C^{I V}}
$$

$$
Q=\frac{F^{I V} x D^{I V}}{f_{3}^{I V}}
$$

Whole body cholesterol synthesis in vivo was measured according to mass isotopomer distribution analysis (MIDA) (18-20). In short, mice were supplemented with $\left[1-{ }^{13} \mathrm{C}\right]$ acetate $(2 \% \mathrm{w} / \mathrm{w}$ in tap water) for 3 days and feces $(1 \mathrm{x}$ daily) and blood spots $(0,8,24$, $32,48,56$ and 72) were collected.

The frequencies of isotopologues of cholesterol molecules in blood spots, i.e., M1 and M3, were used to calculate precursor pool enrichments of cholesterol synthesis. 
These enrichments were used to calculate the distributions of M1 and M3 in the newly synthesized part of cholesterol $\left(\mathrm{M}^{* 1}\right.$ and $\left.\mathrm{M} * 3\right)$.

The ratios $\mathrm{M} 1$ over $\mathrm{M} * 1$ and $\mathrm{M} 3$ over $\mathrm{M} * 3$ gives an estimation of the fractional cholesterol synthesis. The absolute cholesterol was subsequently determined as the product of the fractional synthesis and pool size.

\section{IN VIVO Vldl production and VldL-LIKe emUlgion particle Clearance}

VLDL production was assessed in $\mathrm{PHD}^{-/} \mathrm{LDLr}^{-/-}$and $\mathrm{LDLr}^{-/-}$mice after 8 weeks of a HCD using i.v. injections with Triton WR1339 (Sigma, T8761, $500 \mathrm{mg} / \mathrm{kg}, 6$ mice per genotype) or i.p. with poloxamer 407 (Sigma, 16758, $1000 \mathrm{mg} / \mathrm{kg}, 10 \mathrm{mice}$ per genotype). Blood samples were withdrawn over time for triglyceride (TG) analysis in plasma.

For VLDL clearance, PHD1 ${ }^{-/} \mathrm{LDLr}^{-/}$and $\mathrm{LDLr}^{-/}$mice ( $\mathrm{n}=10 /$ group) were fed a high cholesterol diet (HCD: 0.25\% cholesterol, SDS 824171) for 8 weeks and fasted for 4 hours prior to the experiment and blood was taken via tail snip at $\mathrm{t}=0 \mathrm{~min}$. Glycerol tri $\left[{ }^{3} \mathrm{H}\right]$ oleate $\left(\left[{ }^{3} \mathrm{H}\right] \mathrm{TO} ; 100 \mu \mathrm{Ci}\right)$ and $\left[{ }^{14} \mathrm{C}\right]$ cholesteryl oleate $\left(\left[{ }^{14} \mathrm{C}\right] \mathrm{CO} ; 10 \mu \mathrm{Ci}\right)$ doublelabeled VLDL-like particles were prepared as described earlier (21). To study the in vivo clearance of these particles, mice were fasted for $4 \mathrm{~h}$ and injected intravenously with the emulsion ( $1 \mathrm{mg}$ TG in $200 \mu \mathrm{l}$ emulsion per mouse). Blood samples were taken via tail snip at 2, 5, 10 and 15 min after injections. Subsequently, mice were sacrificed by cervical dislocation and perfused via the heart for $5 \mathrm{~min}$ with ice-cold PBS containing 10U/ $\mathrm{ml}$ heparin. Next, organs were harvested and uptake of $\left[{ }^{3} \mathrm{H}\right] \mathrm{TO}$ and $\left[{ }^{14} \mathrm{C}\right] \mathrm{CO}$-derived radioactivity by the organs was determined and expressed as percentage of the injected dose per gram wet tissue weight.

\section{Antigense dligonucledtide (As0) Injections}

Control or HIF-2 $\alpha$ ASOs were generously provided by ISIS Pharmaceuticals. LDLr ${ }^{-/}$

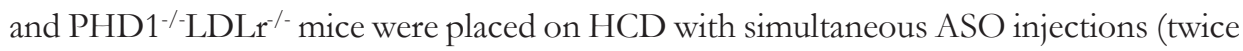
weekly i.p. $25 \mathrm{mg} / \mathrm{kg}$ ). Blood was withdrawn via vena saphena prior to first injections and at 2, 4 and 6 weeks of ASO administration (maximum $50 \mu$ l to avoid anemia effects). 


\section{PLASMA CHOLESTEROL, TRIGLYCERIDES ANALYSIS AND LIPOPROTEIN FRACTIONING}

Plasma was obtained by centrifugation, snap-frozen and stored at $-80^{\circ} \mathrm{C}$ until further use. Plasma total cholesterol (TC) and TG were assessed using standard enzymatic assays (Cholesterol FS’10; Ref: 113009910 021; Diagnostic Systems GmbH, Holzheim, Germany and Triglycerides FS 5' Ecoline; Ref: 157609990 314; Diagnostic Systems GmbH, Holzheim, Germany) automated on the Cobas Fara centrifugal analyzer (Roche).

Hepatic cholesterol and TG were extracted using the Bligh \& Dyer lipid extraction method (15). Pooled plasma samples from $\mathrm{LDLr}^{-/}$and $\mathrm{PHD} 1^{-/} \mathrm{LDLr}^{-/}$mice were used for lipoprotein fractioning on a Superose 6 PC 3.2/30 column (Akta System, Amersham Pharmacia Biotech, Piscataway, NJ). The samples were eluted at a constant flow rate of $50 \mu \mathrm{L} / \mathrm{min}$ in PBS (supplemented with $1 \mathrm{mmol} / \mathrm{L}$ EDTA; pH 7.4). Fractions of $50 \mu \mathrm{l}$ were collected and assayed for TC and TG using the kits described above.

\section{hepatic ChOlegterol, triglyceride, gLYCOGEN AND ATP}

Livers were snapfrozen and stored at $-80^{\circ} \mathrm{C}$ until further use. For cholesterol and TG analysis, approx. $50 \mathrm{mg}$ tissue was homogenized using glass beads in SET buffer $(250 \mathrm{mM}$ sucrose, $2 \mathrm{mM}$ EDTA, $10 \mathrm{mM}$ Tris pH6.8), with 2 subsequent freeze-thaw cycles. Samples were then further homogenized by pulling through $25 \mathrm{G}$ needles repeatedly, followed by another freeze-thaw cycle of the homogenate. Hepatic cholesterol and TG were determined and subsequently corrected for protein content in the homogenate (BCA kit, Thermo Fisher Scientific, Cat. No. 23227).

Hepatic glycogen extraction was performed adjusted from (22). In short, $25 \mathrm{mg}$ of liver was homogenized in $1 \mathrm{M} \mathrm{KOH}$ using glass beads and incubated at $100^{\circ} \mathrm{C}$ for $30 \mathrm{~min}$. Lysates were neutralized adding 0.5 volume of $1 \mathrm{M}$ perchloric acid. Samples were centrifuged at $10000 \mathrm{~g}$ for $1 \mathrm{~min}$ and supernatants were left to precipitate overnight in $95 \% \mathrm{EtOH} / 0.1 \% \mathrm{LiCl}$. Precipitates were washed in $80 \% \mathrm{MeOH} / 0.1 \% \mathrm{LiCl}$ and $80 \% \mathrm{EtOH} / 0.1 \% \mathrm{LiCl}$ in water with centrifugation at $1000 \mathrm{rpm}$ for $2 \mathrm{~min}$ in between. Pellet was left to dry and hydrolyzed to glucose using $1 \mathrm{M} \mathrm{HCl}\left(2 \mathrm{~h}, 100^{\circ} \mathrm{C}\right)$. Upon cooling, the reaction was neutralized with $2 \mathrm{M} \mathrm{NaOH}$. Glucose was then measured using the Glucose (GO) Assay kit (GAGO-20 Sigma).

Hepatic ATP was extracted using Phenol-TE. 20-30mg tissue was taken up in $3 \mathrm{ml}$ PhenolTE and homogenized using glass beads. Per ml homogenate, $200 \mu \mathrm{l}$ chloroform and $150 \mu \mathrm{l}$ de-ionized water was added and upon thorough shaking (20s) and centrifugation $\left(10,000 \mathrm{rpm}, 5 \mathrm{~min}, 4^{\circ} \mathrm{C}\right)$, ATP was measured in the supernatant using the CellTiter-Glo 
Luminescence Cell Viability Assay (Promega G7570).

\section{FLOW CYTOMETRY AND bloOd PARAmeters}

Cells isolated from whole blood, spleen and lymph nodes were analyzed using flow cytometry ( $\mathrm{n}=10$ per group). Spleen and lymph nodes were crushed into single-cell suspensions and blood and spleen were subsequently subjected to erythrocyte lysis. Following specific antibodies were used to detect leucocyte subsets in all tissues: bone marrow stem cells (lin/Sca-1/c-kit; eBioscience, BD), common myeloid progenitors $\left(\mathrm{CD} 16 / 32^{\text {int }}, \mathrm{CD} 34^{\text {int, }} ;\right.$ eBioscience, $\mathrm{BD}$ respectively), granulocyte-macrophage progenitors (CD16/32 high, $\left.\mathrm{CD} 34^{\text {high }}\right)$, erythrocyte-megakaryocyte progenitors (CD16/32 , CD34), leucocytes (CD45 ${ }^{+}$, Biolegend), T cells (CD3 $\varepsilon^{+}$, NK1-1; Miltenyi, eBioscience, resp.), Thelper cells $\left(\mathrm{CD} 4^{+}, \mathrm{BD}\right)$, cytotoxic $\mathrm{T}$ cells $\left(\mathrm{CD} 8 \mathrm{a}^{+}, \mathrm{BD}\right)$, effector $\mathrm{T}$ cells $\left(\mathrm{CD} 44^{\text {high }}\right.$, $\mathrm{CD} 62^{\text {low; }} \mathrm{BD}$, eBioscience, resp.), central memory $\mathrm{T}$ cells $\left(\mathrm{CD} 44^{\text {high }}, \mathrm{CD} 66^{\text {high }}\right)$, naive $\mathrm{T}$ cells (CD44 $\left.4^{\text {low }}, \mathrm{CD} 66^{\text {high }}\right)$, regulatory $\mathrm{T}$ cells $\left(\mathrm{CD} 4^{+}, \mathrm{CD} 25^{\text {high }}\right.$, FoxP $3^{\text {high }}$; eBioscience), $\mathrm{B}$ cells $\left(\mathrm{B} 220^{+} ; \mathrm{BD}\right), \mathrm{NK}$ cells $\left(\mathrm{NK} 1-^{+}\right)$, granulocytes $\left(\mathrm{CD} 11 \mathrm{~b}^{\text {high }}\right.$, Ly6 $\mathrm{G}^{\text {high }} ; \mathrm{BD}$, eBioscience, resp.) and monocytes (CD11 $\mathrm{b}^{\text {high }} \mathrm{Ly} 6 \mathrm{G}^{\text {low }} \mathrm{Ly} 6 \mathrm{C}^{\text {high/intermediate/low; }}$ Miltenyi). Data were acquired using a FACS Canto II and analyzed with FACSdiva software (BD).

For blood parameter analysis, whole blood was diluted 1:10 in Hepes buffer, $\mathrm{pH} 7.45$ (10mM Hepes, $136 \mathrm{mM} \mathrm{NaCl}, 2.7 \mathrm{mM} \mathrm{KCl}, 2 \mathrm{mM} \mathrm{MgCl}_{2}, 0.1 \%$ glucose, $0.1 \%$ BSA) and subsequently measured on the XP3000 Sysmex analyzer (Sysmex, Chuo-ku Kobe, Japan).

\section{QUANTITATIVE RT- PCR (DPCR)}

Total RNA was isolated and transcribed as described (23). QPCR analyses were performed from 10ng cDNA and amplicons were visualized SYBR green using gene specific primer sets (Supplemental Table 2). For hepatic genes $18 \mathrm{~S}$ rRNA and for intestinal samples GAPDH was used as housekeeping gene, insensitive to changes in oxygen levels and PHD1 deficiency. 


\section{HUMAN ATHEROSCLEROSIS}

All experiments were conducted in agreement with the code for proper secondary use of human tissue in the Netherlands (http://www.fmwv.nl). PHD1 protein expression was assessed by immunofluorescence in human carotid autopsy samples $(n=38$, mean age 72 years, 64\% men) representing the following stages of atherosclerosis: intimal thickening, pathological intimal thickening, thick fibrous cap (stable) atheroma, and plaque with intraplaque haemorrhage. After antigen retrieval (target retrieval DAKO), slides were incubated overnight with rabbit anti-human PHD1 antibody (1:100 Novus Biologicals NB100-310), followed by biotin-conjugated donkey-anti-rabbit, streptavidinhorseradish peroxidase (hrp) and Cy3 labelled tyramide signal amplification reagent (Perkin Elmer). Nuclei were visualised with DAPI. PHD1 mRNA expression in 21 thick fibrous cap atheromas (stable) and 23 ruptured plaques was analyzed by microarray derived from human carotid endarterectomy, as described in (10). In short, flanking sites of tissue used for transcriptomics analysis were formalin-fixed for subsequent plaque stage analysis. PHD1 mRNA expression intensities from microarrays was correlated with morphometrically analyzed histological plaque characteristics: plaque size, lipid core, necrotic core ( $\%$ of plaque), haemorrhage ( $\%$ of plaque), microvessels $(\%$ CD 31 of plaque), macrophages ( $\%$ CD 68 of plaque), T-cells ( $\%$ CD3 of plaque). Classification of all used plaques was performed on hematoxylin and eosin (HE) stained slides according to Virmani et al. (11) by two investigators independently (T.L.T. and J.C.S.).

\section{STATISTICAL ANALYSIS}

All data are presented as mean+SEM, with ${ }^{*}$ p-value $<0.05$, **p-value $<0.01$, $*_{* *}$ p-value $<0.0001$. All parameters were analyzed using independent sample tests and were tested for normal distribution using Shapiro-Wilk normality test. Parameters with two groups were compared with student's t-test or Mann-Whitney rank-sum test. In case of more than two groups, parameters were analyzed using one-way ANOVA followed by Bonferroni's Multiple Comparison Test or Kruskal-Wallis rank-sum test, followed by Dunn's post-hoc testing. Time course experiments were analyzed using repeated measured (mixed model) ANOVA, followed by Bonferroni post-tests upon confirmation of homogeneity of variances using Levene's (IBM SPSS statistics 22). Correlation analysis was performed using Spearman bivariate correlation analysis (IBM SPSS statistics 22). 


\section{RESULTS}

\section{PHDI DEFICIENCY REDUCES PLAQUE FORMATION, PLAQUE hYPOXIA AND PLASMA CHOLESTEROL LEVELS}

In keeping with our hypothesis, PHD1 deficiency reduced plaque size and necrotic core content compared to $\mathrm{LDLr}^{-/}$mice on high cholesterol diet (HCD) (Figure 1 A). Hypoxic (pimonidazole-positive) area was reduced in the atherosclerotic plaques of $\mathrm{PHD}^{-/} \mathrm{LDLr}^{-/}$mice. This alleviation in plaque hypoxia was independent of the reduction in plaque size and macrophage content (Figure 1A), suggesting PHD1 deficiency reduced macrophage oxygen consumption as shown before in other cell types (8).

Figure 1

A
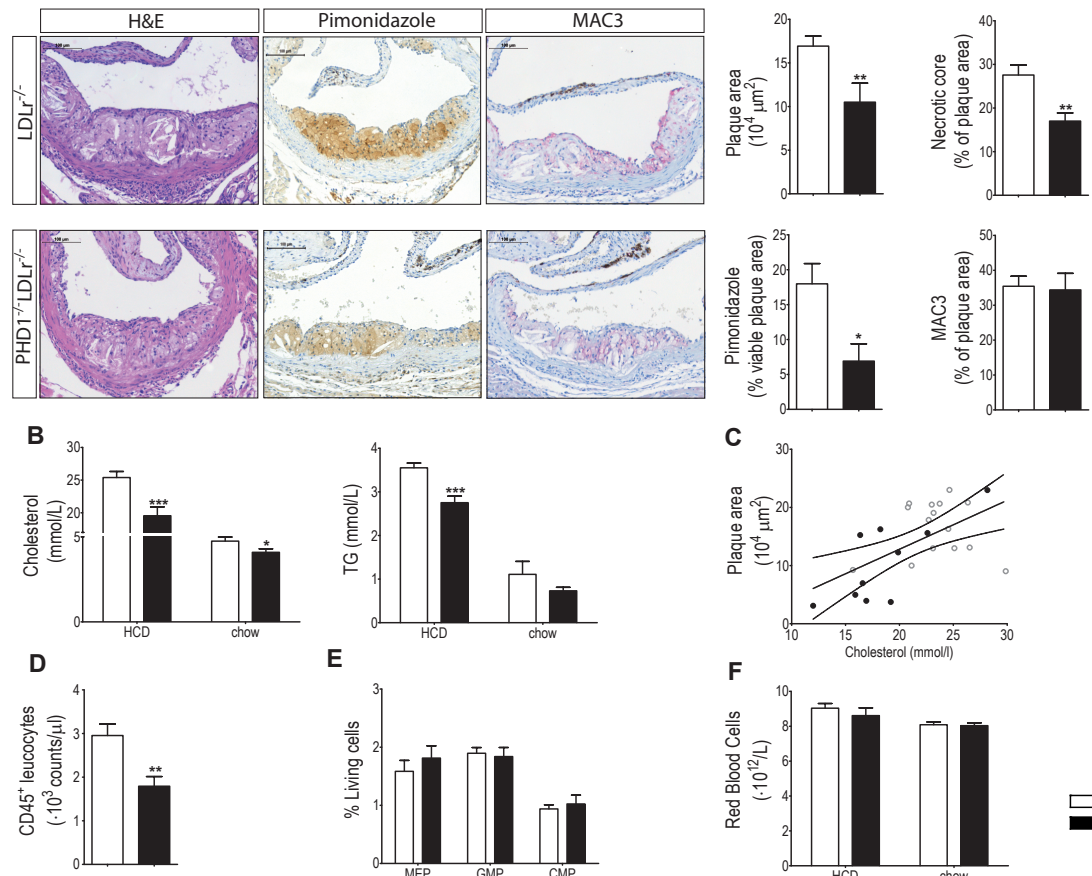

E

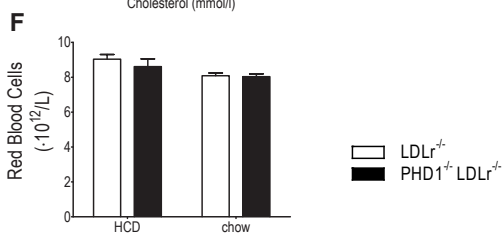

Figure 1: PHD1 deficiency reduces plaque formation and plaque hypoxia, but also plasma cholesterol levels.

A. Representative pictures of aortic roots (bematoxylin and eosin H\&E, pimonidazole and MAC3) and quantifications of plaque size and necrotic core as well as plaque hypoxia and macrophage content of PHD1/LDLr/ mice $(n=10)$ and LDLr/- $(n=17)$ after 8 weeks of HCD. B. Plasma cholesterol and triglyceride levels after 8 weeks of high cholesterol diet and on chow. C. Linear regression analysis of plasma cholesterol and plaque size shown for both genotypes (grey open circles for LDL $r^{/-}$and closed black circles for PHD1/ LDL $r^{-/}, r^{2}=0.3388$, $\left.p=0.0023\right)$. D. Total CD45 lencocyte 
count and E. flow cytometry of hematopoietic progenitors megakaryocyte-erythroid progenitor (MEP), granulocytemacrophage progenitor (GMP) and common myeloid progenitor (CMP) isolated from bone marrow of LDLr/- (white bars) and PHD 1\% mice (black bars). F. Red blood cell count in whole blood upon 8 weeks of HCD and on chow. White bars represent $L D L r^{\circ} /$ and black bars represent $P H D 1^{-1}-L D L r^{\prime-~ m i c e . ~}$

Surprisingly, $\mathrm{PHD}^{-/} \mathrm{LDLr}^{-/}$mice showed reduced plasma cholesterol and triglyceride (TG) levels (Figure 1B), despite similar food and hence cholesterol intake and body weight gain (Supplemental Figure 1A). Cholesterol levels correlated with atherosclerotic plaque area (r2=0.3388, $\mathrm{p}=0.0023$, Figure 1C). Additionally, $\mathrm{PHD}^{-/} \mathrm{LDLr}^{-/}$mice presented with significantly reduced leucocyte count in blood, spleen and lymph nodes across all sub-populations (Figure 1D, Supplemental Figure 2, lymph node and spleen data not shown). The same trend was observed in mice on chow diet (data not shown). This effect was not due to aberrant leucocyte differentiation, as myeloid progenitors in bone marrow were not changed (Figure 1E, Supplemental Figure 1B). Although erythropoiesis is HIF-dependent, red bone marrow erythropoietic progenitors, blood cell count or morphology, hemoglobin, hematocrit, blood lactate and $\mathrm{pH}$, were unchanged on HCD and chow diet (Figure 1F, Supplemental Figure 1C-F). Unaltered plasma thyroid stimulating hormone (TSH) levels show that PHD1 deficiency does not result in an obvious hyperthyroidism, thereby enhancing metabolic rate and cholesterol usage (Supplemental Figure 1G). This data points towards a direct effect of PHD1 on cholesterol metabolism.

heMATOPOIETIC PhDI DEFICIENCY DOES NOT AFFECt AtheROSCLEROSIS DEVELOPMENT AND MARGINALLY LOWERS PLASMA CHOLESTEROL LEVELS

In order to differentiate between the effects of cholesterol and inflammation on atherosclerosis development, bone marrow from wild-type (WT) or PHD1 ${ }^{-/}$bone marrow was transplanted into $\mathrm{LDLr}^{-/}$mice. Hematopoietic PHD1 deficiency in $\mathrm{LDLr}^{-/}$recipient mice did not significantly affect plasma cholesterol or TG levels (Figure 2A, PHD1 $1^{-/}$bone marrow indicated by $\left(^{(-/}\right)$, left two bars). Only upon stromal PHD1 deficiency (PHD1 ${ }^{-/} \mathrm{LDLr}^{-/}$mice with ${ }^{-/}$bone marrow), plasma cholesterol and TG levels were significantly lowered compared to $\mathrm{LDLr}^{-/}$mice receiving wt bone marrow (Figure $2 \mathrm{~A}$, right two bars).

Also, hematopoietic deficiency of PHD1 in $\mathrm{LDLr}^{-/-}$recipients did not significantly affect leucocyte count, plaque hypoxia or atherosclerosis development (Figure 2B,C), suggesting that mainly the stromal and not the hematopoietic fraction contributes to PHD1 deficiency-associated changes in cholesterol metabolism, which might subsequently affect leucocyte count and plaque hypoxia and development. In accordance, PHD1 mRNA and immunoreactivity expression in human atherosclerotic plaques did not 
correlate with plaque phenotype (intraplaque hemorrhage, lipid core, necrotic core T cells or $\mathrm{CD} 31^{+}$vessels) (Supplemental Table 1, Supplemental Figure 3), underpinning the importance of PHD1 expression in regulation of cholesterol levels.

Figure 2

A

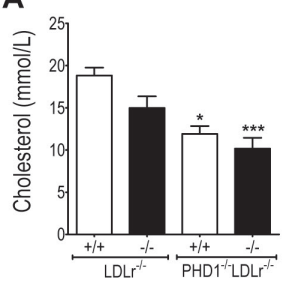

C
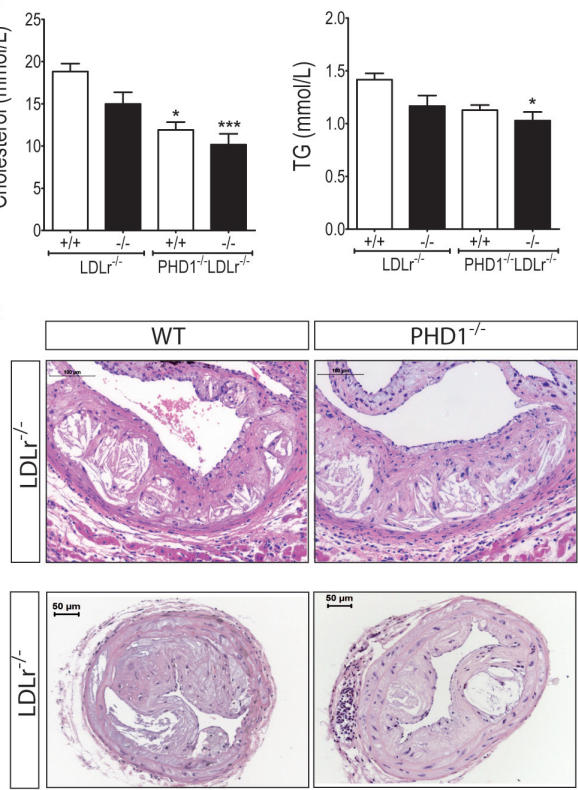

B
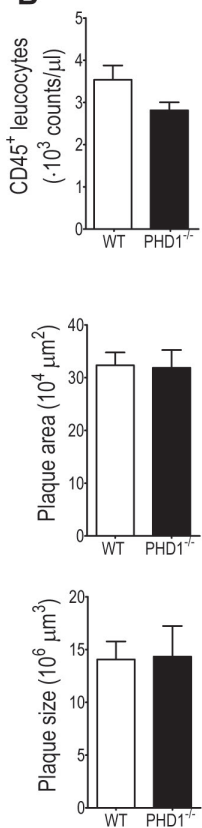
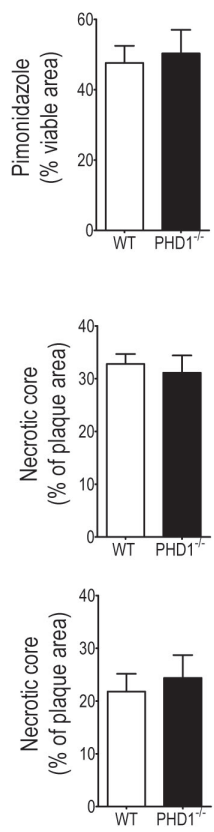

Figure 2: Hematopoietic PHD1 deficiency does not affect atherosclerosis development.

A. Plasma cholesterol and triglyceride levels upon hematopoietic PHD1 deficiency (-/-, black bars) or wildtype (+/+, white bars) in LDLr/- recipients ( $n=20 / 20$,) or PHD1/-LDLr/- recipients $(n=8 / 8)$. B. Leucocyte count and plaque bypoxia in whole blood of LDLr/- recipients reconstituted with wild-type (WT $n=20)$ or PHD1\% $(n=20)$ bone marrow. C. Representative pictures (H\&E) of atherosclerotic plaques in aortic roots (upper panel, $n=20 \mathrm{WT}$ and $n=20$ PHD 1\%) and carotid artery (lower panel $n=20 \mathrm{WT}$ and $15 \mathrm{PHD} 1^{-}$) with quantifications of plaque size and necrotic core content of $L D L r^{\prime}-$ recipients with $W T$ (black bars) or PHD1\% (wbite bars) reconstituted bone marrow. 


\section{hepatic ChOLEgterol and tg Metabolism IS NOT AFFECted bY PhDI DEFICIENCY}

Next, the liver as the main regulator of lipid metabolism was further studied. Hepatocyte cell size, hepatic inflammation, hypoxia and glycogen content were similar in $\mathrm{PHD}^{-/} \mathrm{LDLr}^{-/}$mice and littermate controls, with a slight reduction in liver weight (Supplemental Figure 4A-C). The VLDL pool could be held accountable for the lower plasma cholesterol levels observed in $\mathrm{PHD}^{-/} \mathrm{LDLr}^{-/}$mice on HCD and on chow diet (Figure 3A, Supplemental Figure 4D,E), while high density lipoprotein (HDL)cholesterol and HDL-apoAI concentrations (data not shown) remained unchanged.

We therefore investigated hepatic VLDL-TG production and clearance in PHD1\% $\mathrm{LDLr}^{-/}$mice compared to controls. Hepatic VLDL-TG production was similar in PHD1 /-LDLr/- mice compared to controls using two different lipoprotein lipase inhibitors, Poloxamer 407 (Figure 3B) and Triton WR1339 (Supplemental Figure 4F,G). To assess VLDL clearance, we generated glycerol tri $\left[{ }^{3} \mathrm{H}\right]$ oleate and $\left[{ }^{14} \mathrm{C}\right]$ cholesteryl oleate $\left(\left[{ }^{3} \mathrm{H}\right] \mathrm{TO}\right.$, $\left.\left[{ }^{14} \mathrm{C}\right] \mathrm{CO}\right)$ double-labeled VLDL-like particles, allowing tracing of the selective clearance of triglycerides via lipoprotein lipase (LPL)-mediated delipidation $\left(\left[{ }^{3} \mathrm{H}\right] \mathrm{TO}\right)$, in addition to clearance of cholesteryl ester-containing remnant particles $\left(\left[{ }^{14} \mathrm{C}\right] \mathrm{CO}\right)$. Plasma clearance of $\left[{ }^{3} \mathrm{H}\right] \mathrm{TO}$ and $\left[{ }^{14} \mathrm{C}\right] \mathrm{CO}$ was unaltered (Figure 3C, Supplemental Figure $4 \mathrm{H}$ ). Interestingly, the hepatic uptake of ${ }^{14} \mathrm{C}$ activity (reflecting hepatic remnant particle uptake) was enhanced in $\mathrm{PHD}^{-/} \mathrm{LDLr}^{-/}$mice compared to $\mathrm{LDLr}^{-/}$mice (Figure 3D, right graph). Hepatic expression of SR-B1 was indeed increased, possibly explaining enhanced cholesterol uptake in the liver (Figure 3E). However, cholesterol did not accumulate in the liver, as witnessed by the unchanged hepatic cholesterol content (Figure 3F) and oil red O-stainable lipid content (Supplemental Figure 4I). In line, hepatic expression of HMG-CoA reductase, a rate-limiting enzyme in cholesterol synthesis and negatively regulated by hepatic lipid accumulation (24), was unchanged. Accordingly, unaltered hepatic acetyl-CoA levels, a cholesterol precursor (Figure 3G), reflected unchanged cholesterol synthesis in $\mathrm{PHD}^{-/} \mathrm{LDLr}^{-/-}$mice. Also, whole body cholesterol synthesis, measured by $\left[{ }^{13} \mathrm{C}\right]$ acetate incorporation into the cholesterol pool was unaltered (Figure 3H). Thus, internalized cholesterol is probably rapidly excreted by the liver in $\mathrm{PHD}^{1 /-} \mathrm{LDLr}^{-/}$mice by either fast clearance from the liver via the bile or by release back into the circulation. 
Figure 3
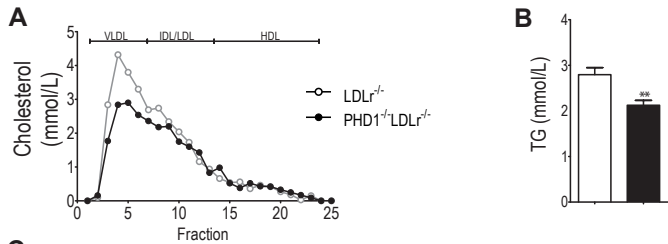

C
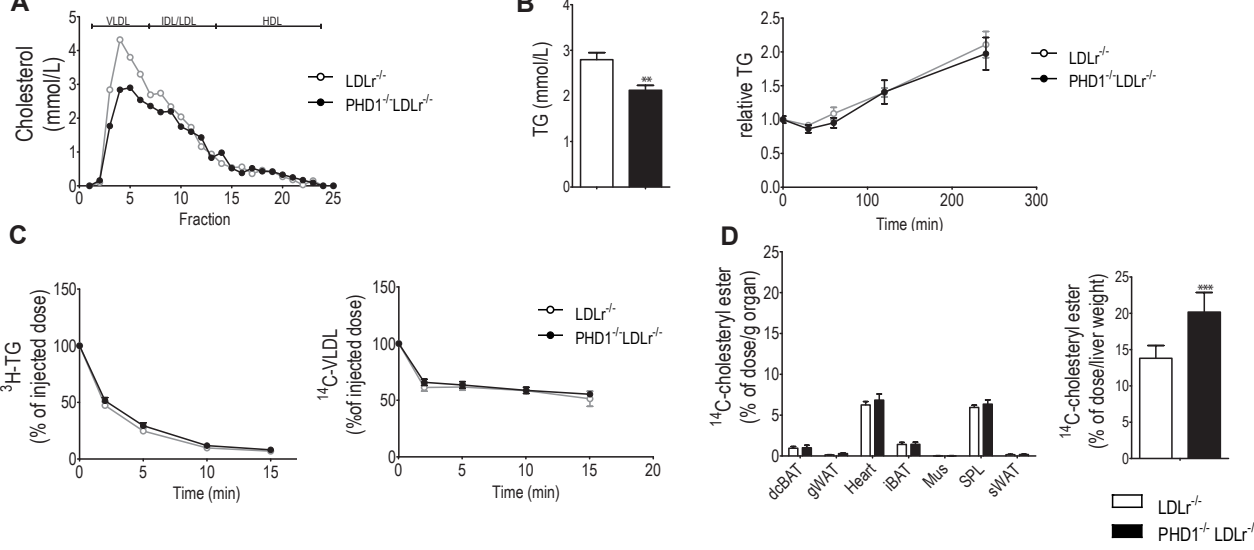

E

$\mathbf{F}$

G

H
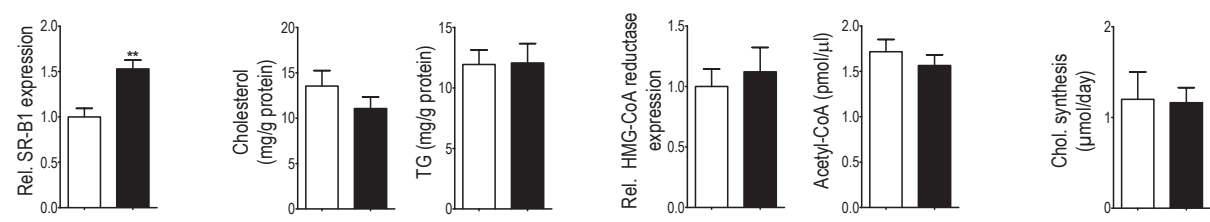

Figure 3: PHD1 deficiency does not influence hepatic TG and cholesterol metabolism

All data presented in this figure were obtained after 8 weeks of HCD. A. Lipoprotein fractionation of pooled plasma samples from PHD1/LDLr/- mice and LDLr/ controls (pooled samples $n=3$ ). B. VLDL-TG packaging upon poloxamer 407 injection. Results are presented relative to 1, as plasma TG levels were again decreased on baseline (left graph) in PHD1-LDLr- mice compared to littermate controls ( $n=10 /$ group). C. Glycerol tri $\left.{ }^{3} \mathrm{H}\right]$ oleate-labeled emulsion particle clearance representing TG clearance (left) and cholesteryl ester clearance over time (right) $(n=10 /$ group). D. Accumulation of $\left[{ }^{14} \mathrm{C}\right]$ cholesteryl oleate-labeled remnants analyzed in various indicated tissues (left graph). Liver accumulation of the particles (right graph) is corrected for total liver weight, as liver were again lighter in PHD1 ${ }^{\prime} \mathrm{L} D \mathrm{Lr}$ / - mice compared to controls (data not shown) ( $n=10 /$ group). E. Hepatic SR-B1 expression relative to LDLr 1- control. F. Hepatic cholesterol and TG levels as well as G. relative HMG-Co A reductase expression and acetyl-Co A content in the liver ( $n=10 /$ group). $H$. Whole body cholesterol synthesis as analyzed as $\left[{ }^{13} \mathrm{C}\right]$-acetate incorporation into plasma cholesterol levels, corrected for differences in plasma cholesterol pool ( $n=10 /$ group). White bars represent LDLr 1- and black bars represent PHD ${ }^{-1} L D L r^{*-}$ mice. 


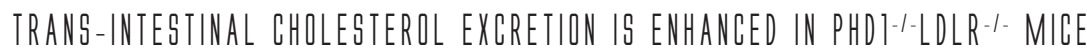

Plasma cholesterol levels are the resultant of input and output pathways. Since cholesterol synthesis and subsequent VLDL secretion were unaltered, while plasma cholesterol levels were reduced, another cholesterol flux must have changed. We therefore performed a whole body cholesterol flux analysis using stable isotope labeling of cholesterol in vivo (12).

Fecal excretion of neutral sterols was enhanced in $\mathrm{PHD} 1^{-/} \mathrm{LDLr}^{-/}$mice on chow (Figure 4A). This effect was less pronounced after 8 weeks on HCD, as the high dietary cholesterol load potentially obscured the measurement (Figure 4B). Thus, PHD1 deficient mice presented with excretion of neutral sterols, such as cholesterol. Bile duct cannulations at the end of the experiment showed unaltered bile flow between the two groups upon HCD (Figure 4C). Surprisingly, biliary cholesterol excretion (measured as cholesterol concentration in bile) was even lower in $\mathrm{PHD}^{-/-\mathrm{LDLr}^{-/}}$mice (Figure 4D) and bile acid concentration in bile was unaffected in PHD1 deficient mice (Figure 4E). Interestingly, hepatic PPAR $\alpha$ expression was upregulated, in line with upregulation of some of the target genes, including hepatic ABCG5/8 mRNA expression (Figure 4F right panel), which has been directly linked to enhanced biliary cholesterol excretion (25). However, hepatic levels of ATP, the main co-factor for ABCG5/8 activation, were significantly reduced in PHD1 deficient livers (Figure 4G), suggesting reduced ATP-binding cassette protein function to explain reduced biliary cholesterol excretion. Biliary cholesterol excretion could hence not explain the reduction in plasma cholesterol levels in PHD1 deficient mice. Additionally, even though biliary cholesterol excretion is reduced, cholesterol did not accumulate in the liver and must be released back into the circulation (despite lower plasma cholesterol levels). All effects were independent of dietary cholesterol intake (Figure 4H). Increased fecal neutral sterol excretion despite of decreased biliary secretion and slightly increased intestinal cholesterol absorption (Figure 4I) can only be accounted for by increased non-biliary cholesterol excretion. Indeed this pathway, termed trans-intestinal cholesterol excretion (TICE), was increased in $\mathrm{PHD}^{-/} \mathrm{LDLr}^{-/}$mice, likely accounting for the reduced plasma cholesterol levels (Figure 4J). Thus, in vivo analysis of cholesterol homeostasis showed a strong contribution of non-biliary, extra-hepatic cholesterol excretion to PHD1-mediated lower plasma cholesterol levels. 
Figure 4
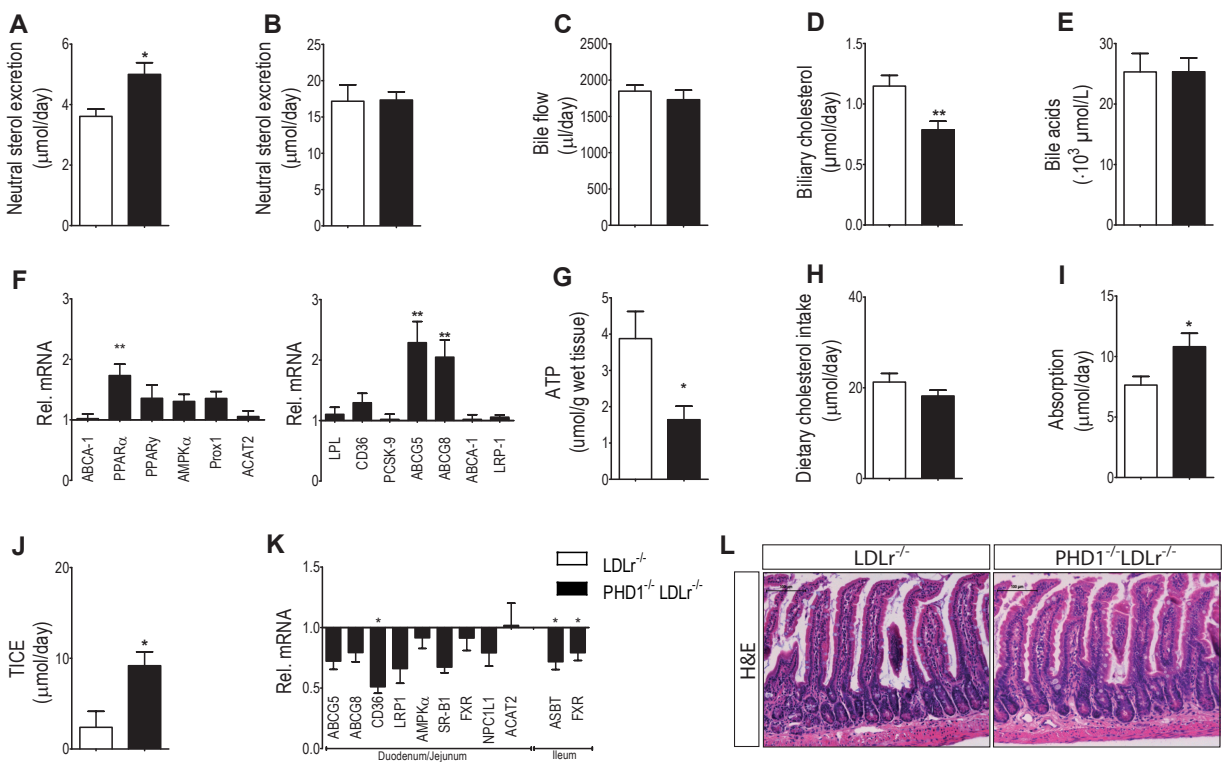

Figure 4: Trans-intestinal cholesterol excretion is enhanced in $\mathrm{PHD1}^{-/-\mathrm{LDLr}^{-/} \text {mice }}$

If not indicated otherwise, data was acquired upon 8 weeks of HCD feeding $(n=10 /$ group). A. Neutral sterol excretion on chow and B. after 8 weeks of HCD. C. Bile flow upon 30min of bile cannulation and D. Biliary cholesterol excretion, corrected for bile flow in mice. E. Total bile acid content in bile. F. Relative $m R N A$ expression of hepatic genes in PHD1 deficient mice, presented relative to LDLr/- littermate controls $(n=10 /$ group). G. Hepatic ATP content. H. Dietary cholesterol intake monitored over 10 days after 8 weeks of HCD. I. Cholesterol absorption presenting the sum of dietary cholesterol absorption and re-absorption. J. Trans-intestinal (non-biliary) cholesterol excretion in PHD1 deficient mice and controls, as calculated based on dietary cholesterol input and biliary cholesterol excretion subtracting cholesterol absorption and neutral fecal neutral sterol excretion. $K$. Relative $m R N A$ expression of various genes in duodenum/jejunum and ileum of PHD1 deficient mice on chow. Data is presented relative to littermate LDLr deficient controls $\left(n=6 /\right.$ group). L. Representative pictures of duodenums of PHD1-LDL $r^{r-a}$ and LDLr/ controls, presenting similar villi length, epithelial cell alignment and inflammation. White bars represent $L D L r^{\prime-}$ and black bars represent PHD 1/-LDLr/- mice.

The molecular processes underlying TICE have only been partly revealed (26). Current thoughts are that TICE may be regulated at the intestinal level by the cholesterol transporters Niemann-Pick C1-like 1 (NPC1L1), ATP-binding cassette sub-family G member 5 and 8 (ABCG5/8) or other VLDL receptors and cholesterol transporters. Intestinal gene expression analysis, however, did not reveal any changes or even decreased expression of known regulators of TICE in $\mathrm{PHD}^{-/} \mathrm{LDLr}^{-/}$mice compared to $\mathrm{LDLr}^{-/}$controls (Figure 4K). Likewise, intestines of $\mathrm{PHD}^{-/} \mathrm{LDLr}^{-/}$mice did not show any morphological abnormalities such as enterocyte toxicity and inflammation, 
confirming a viable and functional intestinal barrier (representative pictures, Figure 4L).Thus, PHD1 deficiency stimulated cholesterol excretion through strong elevation of non-biliary cholesterol efflux, without changes in known intestinal cholesterol transporter expression or intestinal histology.

\section{PhDI DEFICIENCY-MEDIATED CHOLESTEROL LOWERING IS HIF-2A INDEPENDENT}

PHD1 deficiency results in HIF-2 $\alpha$ rather than HIF-1 $\alpha$ stabilization (27). Therefore, we investigated a causal role for HIF- $2 \alpha$ stabilization in cholesterol lowering in $\mathrm{PHD}^{-/} \mathrm{LDLr}^{-/}$mice using antisense oligonucleotide (ASO) mediated knockdown of $\mathrm{HIF}-2 \alpha$ in $\mathrm{LDLr}^{-/}$and PHD1 ${ }^{-/-} \mathrm{LDLr}^{-/}$mice. ASO injections twice weekly resulted in a specific HIF- $2 \alpha$ knockdown of $80-90 \%$ in both liver and intestines (Supplemental Figure $5 \mathrm{~A}, \mathrm{~B}$ expression data expressed relative to control ASO HIF-2 $\alpha$ expression). Plasma cholesterol levels were again lower in $\mathrm{PHD}^{-/} \mathrm{LDLr}^{-/}$mice prior to start of injections (Figure 5A). However, HIF-2 $\alpha$ knockdown in livers and intestines only increased plasma cholesterol levels in $\mathrm{LDLr}^{-/}$, but not in $\mathrm{PHD}^{-/} \mathrm{LDLr}^{-/-}$mice (Figure 5B,C). As reported previously (28), hepatic HIF-2 $\alpha$ knockdown resulted in overt anemia after 4 weeks (Figure 5D LDLr ${ }^{-/}$recipients, Supplemental Figure 5C $\mathrm{PHD}^{-/-} \mathrm{LDLr}^{-/}$). As cholesterol was already raised prior to anemia, it is unlikely that cholesterol levels in the first 4 weeks were influenced by anemia. Thus, the cholesterol lowering observed in PHD1 deficient mice is not mediated by HIF-2 $\alpha$ stabilization in livers and intestines.

Figure 5
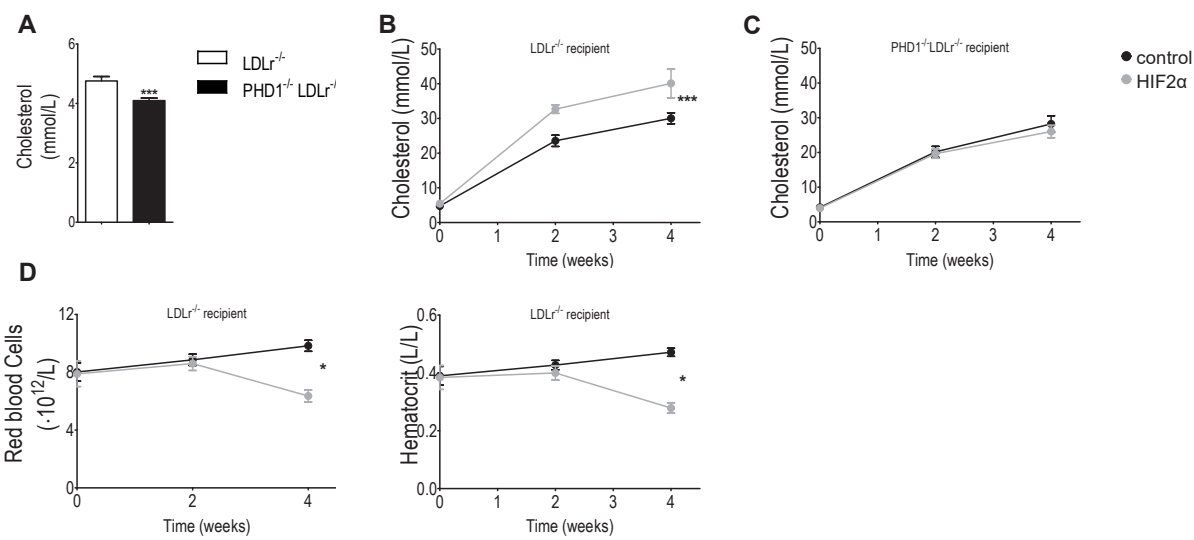

Figure 5: HIF-2 $\alpha$ stabilization does not mediate cholesterol lowering

A. Plasma cholesterol levels in PHD1/LDLr/- mice (black bar) and controls (white bar) prior to start of diet and ASO injections. B. plasma cholesterol levels upon HIF-2a ASO (grey line) and control ASO (black line) in LDL $r^{-1}$ and C. PHD 1/-LDLr/- recipient mice. D. Red blood cell count (left graph) and hematocrit (right graph) over time of HIF-2a ASO injections (grey lines) in LDL $r^{/-}$recipients. 


\section{DISCUSSION}

This study reveals a novel link between two crucial pathways in energy metabolism: oxygen sensing and cholesterol metabolism. Mice deficient in the oxygen sensor PHD1 unexpectedly presented with reduced plasma cholesterol levels and reduced atherogenesis. As PHD1 deficiency in the hematopoietic compartment did not significantly affect atherosclerosis development or leucocyte counts, stromal PHD1 deficiency in whole body $\mathrm{PHD}^{-/} \mathrm{LDLr}^{-/}$mice is likely the dominant factor reducing cholesterol levels, atherogenesis and leucocyte counts. PHD1 deficiency associated cholesterol-lowering appeared to be HIF- $2 \alpha$ independent. While hepatic cholesterol handling was marginally altered and biliary secretion decreased, net cholesterol excretion increased due to strong elevation of non-biliary cholesterol efflux (TICE). This phenomenon likely explains the normalization of plasma cholesterol seen in PHD1 deficiency.

Interest in alternative approaches to cholesterol-lowering therapy is growing, especially after a disappointing translation of HDL-cholesterol raising strategies from preclinical to human cardiovascular disease $(29,30)$. PHD1 inhibition may be a valuable addition to current lipid-lowering therapy, as cholesterol-lowering is independent of HMG-CoA reductase and LDLr, the main point of action of statins and Proprotein convertase subtilisin/kexin type 9 (PCSK9)-inhibitors, respectively. Importantly, general inhibition of all PHDs by roxadustat and GSK1278863 in humans mirrored the cholesterollowering effect seen in PHD1 deficient mice $(31,32)$. Together, these data show a strong relevance of PHD also for human cholesterol metabolism, as well as its therapeutic potential.

However, neither the mechanisms underlying the PHD-mediated changes in plasma cholesterol levels in humans nor the responsible prolyl hydroxylase isoform has been identified. Interestingly, aforementioned PHD pan inhibitors, are thought to preferentially block the PHD2 isoform (33). In fact, murine hypomorphism of PHD2 also lowers plasma cholesterol levels on chow (34), although the underlying mechanism is unknown. In contrast to PHD1 and PHD3, PHD2 germline deficiency is embryonically lethal as it is the main driver of HIF-dependent angiogenesis and erythropoiesis $(35,36)$, which may disqualify it as a drug target for cholesterol lowering.

The selective PHD2 inhibitors described above are advancing to phase 3 clinical trials as erythropoietin-stimulating agents to alleviate anemia related to chronic kidney failure (37). However, in subjects with normal erythrocyte counts, raising erythropoiesis and elevated hematocrit could potentially thwart the beneficial cardiovascular effects of cholesterol lowering by possible effects on thrombosis (38-40). Here, no alterations 
in erythropoiesis were observed in $\mathrm{PHD} 1^{-/} \mathrm{LDLr}^{-/}$mice on chow diet or $\mathrm{HCD}$, as described earlier (8). In addition, HDL-cholesterol was unchanged in PHD1 ${ }^{-1 / \mathrm{LDLr}^{-1}}$ mice, while being lowered in both PHD2 hypomorphism (34) and in human subjects $(31,32)$. Also HDL-apoAI concentration in plasma was unaltered in PHD1 deficient mice. Of course, the effect of overall lipoprotein reduction mediated by PHD2 inhibition on atherosclerosis or cardiovascular outcome in humans has not yet been established. Nevertheless, specific targeting of PHD1 may be a more attractive approach avoiding potential cardiovascular side effects associated with raised hematocrit and HDL and HDL-cholesterol lowering.

Our results suggest a link between cholesterol metabolism on the one hand and hypoxia and/or hydroxylation of the key PHD targets HIF-1 $\alpha$ and HIF-2 $\alpha$, on the other hand. As hypoxia naturally inactivates the enzymatic activity of PHDs resulting in HIF- $\alpha$ stabilization, one would expect hypoxia and HIF signaling to lower plasma cholesterol. In contrast to this hypothesis, mice exposed to continuous (41) or intermittent hypoxia have enhanced serum cholesterol (42), while reoxygenation of hypercholesterolemic mice was unable to lower plasma cholesterol (4). Although, the murine lipoprotein profile in hypercholesterolemia does not necessarily reflect human lipoprotein profiles, the same was observed in humans. Continuous positive airway pressure prevented intermittent hypoxia in sleep apnea patients and reduced, not enhanced, serum cholesterol levels in one randomized clinical trial (43). Conversely, three other randomized controlled trials failed to show any changes, suggesting an ambiguous effect of systemic oxygen on cholesterol metabolism (44-46).

Our study shows that HIF signaling does not explain the cholesterol lowering seen in PHD1 deficient mice as knockdown of hepatic and intestinal HIF-2 $\alpha$ increased plasma cholesterol levels in LDLr/- mice, but did not alter cholesterol levels in PHD1 deficient mice. Intriguingly, this is in contrast to earlier data, reporting dramatically enhanced plasma cholesterol levels upon HIF $\alpha$ stabilization obtained by postnatal hepatic von Hippel Lindau (vHL)-deletion. Effects were mainly dependent on HIF- $2 \alpha$-mediated limitation of bile acid metabolism and biliary cholesterol clearance (41). While biliary cholesterol clearance was also reduced in PHD1 deficient mice, plasma cholesterol levels were decreased. Although both models utilized acute vHL/HIF-2 $\alpha$ knockdown via tamoxifen or ASO, hepatic vHL deletion resulted in lethality due to very severe liver dysfunction. Intriguingly, intestinal or myeloid vHL knockdown in the same study did not alter cholesterol metabolism (41). In our hands, however, simultaneous hepatic and intestinal HIF- $2 \alpha$ knockdown resulted in increased plasma cholesterol levels in $\mathrm{LDLr}^{-/}$controls, potentially pointing towards a cross-talk between liver and intestines in 
HIF signaling and cholesterol metabolism. Thus, interference with complete hypoxic/ HIF signaling via systemic hypoxia or vHL deletion might reflect other pathways then relatively subtle HIF skewing in PHD1 deficient mice.

Additionally, other hydroxylation targets of PHDs might be involved in the cholesterollowering seen in PHD deficiency models, and these targets are likely in non-myeloid sites, given the lack of effect in bone marrow transplantation experiments. Multiple hydroxylation targets have been identified, the most prominent being Inhibitory kappaB kinase-beta (IKK $\beta$ ) (47). If acting through IKK $\beta$ and subsequent nuclear factor kappa $B$ $(\mathrm{NF} \varkappa \mathrm{B})$ activity, PHD1 deficiency would be expected to enhance $\mathrm{NF} \varkappa \mathrm{B}$ activity resulting in reduced plasma cholesterol levels. However, mice with highly active NFxB (48) showed enhanced plasma cholesterol levels, while hampered $\mathrm{NF} \varkappa \mathrm{B}$ activity resulted in reduced plasma cholesterol (49). These studies likely exclude IKK $\beta$ and NFxB as mediators of PHD-driven changes in cholesterol metabolism. Taken together, while there is evidence for hypoxia and mainly HIF-2 $\alpha$-mediated hyperlipidemia and hypercholesterolemia, PHD inhibition attenuates hypercholesterolemia via alternative, yet unknown molecular mechanisms.

\section{CONCLUSIONS}

This study reveals a novel link between PHD and cholesterol metabolism, whereby PHD1 inhibition lowers plasma cholesterol through increased non-biliary cholesterol clearance and reduces atherosclerosis. This effect is likely hypoxia-, HIF- and NFuBindependent and seems to affect a different cholesterol lowering pathway as compared to statins or PCSK9 inhibitors. PHD1 specific inhibition might therefore present an attractive therapeutic strategy in hypercholesterolemia, in particular without affecting HDL-cholesterol or erythropoiesis and subsequent side effects, seen after pan-PHD or PHD2-only inhibition. 


\section{FUNDING}

This work was supported by the Cardiovascular research Institute [CARIM PhD fellowship to TLT], the Netherlands Scientific Organization [NWO VENI, tot JCS]; the Dutch Heart Foundation (NHS2009T038 to PCNR); 'the Netherlands CardioVascular Research Initiative: the Dutch Heart Foundation, Dutch Federation of University Medical Centers, the Netherlands Organisation for Health Research and Development and the Royal Netherlands Academy of Sciences' (CVON2011-19, to PCNR]; by the Belgian Science Policy [IAP P7/03 to PC]; long-term Methusalem funding by the Flemish Government [to PC], and grants from the FWO - Flemish Government [G.0595.12.N, G.0671.12N tot PC], and the National Institutes of Health [HL127930 to EAF].

\section{DISCLOSURES}

Relationships to industry do not exist for EM, JAFD, TLT, BMET, MRB, THvD, MJG, LJD, SHRM, MM, EAF, EB, MJAPD, PC, AKG and JCS. PCNR received research funding from MSD (unrelated to this work). GH is employed by ISIS Pharmaceuticals, supplying the ASOs used in this study. 


\section{REFERENCES}

1. The Lancet. The global burden of disease study 2010 [Internet]. The Lancet. 2012 [cited 2013 May 1]. Available from: http://www.thelancet.com/themed/global-burden-of-disease

2. Sluimer JC, Gasc J-M, van Wanroij JL, Kisters N, Groeneweg M, Sollewijn Gelpke MD, et al. Hypoxia, hypoxia-inducible transcription factor, and macrophages in human atherosclerotic plaques are correlated with intraplaque angiogenesis. J Am Coll Cardiol. 2008 Apr 1;51(13):1258-65.

3. Bjornheden T, Levin M, Evaldsson M, Wiklund O. Evidence of Hypoxic Areas Within the Arterial Wall In vivo. Arterioscler Thromb Vasc Biol. 1999 Apr 1;19(4):870-6.

4. Marsch E, Theelen TL, Demandt JAF, Jeurissen M, van Gink M, Verjans R, et al. Reversal of Hypoxia in Murine Atherosclerosis Prevents Necrotic Core Expansion by Enhancing Efferocytosis. Arterioscler Thromb Vasc Biol. 2014 Sep 25;34(12):2545-53.

5. van der Valk FM, Sluimer JC, Vöö SA, Verberne HJ, Nederveen AJ, Windhorst AD, et al. In vivo Imaging of Hypoxia in Atherosclerotic Plaques in Humans. JACC Cardiovasc Imaging. 2015 Nov 5;8(11):1340-1.

6. Epstein AC, Gleadle JM, McNeill LA, Hewitson KS, O’Rourke J, Mole DR, et al. C. elegans EGL-9 and mammalian homologs define a family of dioxygenases that regulate HIF by prolyl hydroxylation. Cell. 2001 Oct 5;107(1):43-54.

7. Appelhoff RJ, Tian Y-M, Raval RR, Turley H, Harris AL, Pugh CW, et al. Differential function of the prolyl hydroxylases PHD1, PHD2, and PHD3 in the regulation of hypoxia-inducible factor. J Biol Chem. 2004 Sep 10;279(37):38458-65.

8. Aragonés J, Schneider M, Van Geyte K, Fraisl P, Dresselaers T, Mazzone M, et al. Deficiency or inhibition of oxygen sensor Phd1 induces hypoxia tolerance by reprogramming basal metabolism. Nat Genet. 2008 Feb;40(2):170-80.

9. von der Thüsen JH, van Berkel TJ, Biessen EA. Induction of rapid atherogenesis by perivascular carotid collar placement in apolipoprotein E-deficient and low-density lipoprotein receptor-deficient mice. Circulation. 2001 Feb 27;103(8):1164-70.

10. Goossens P, Gijbels MJJ, Zernecke A, Eijgelaar W, Vergouwe MN, van der Made I, et al. Myeloid type I interferon signaling promotes atherosclerosis by stimulating macrophage recruitment to lesions. Cell Metab. 2010 Aug 4;12(2):142-53.

11. Virmani R, Kolodgie FD, Burke AP, Farb A, Schwartz SM. Lessons from sudden coronary death: a comprehensive morphological classification scheme for atherosclerotic lesions. Arter Thromb Vasc Biol. 2000/05/16 ed. 2000;20(5):1262-75.

12. van der Veen JN, van Dijk TH, Vrins CLJ, van Meer H, Havinga R, Bijsterveld K, et al. Activation of the liver X receptor stimulates trans-intestinal excretion of plasma cholesterol. J Biol Chem. 2009 Jul 17;284(29):19211-9.

13. Neese RA, Faix D, Kletke C, Wu K, Wang AC, Shackleton CH, et al. Measurement of endogenous synthesis of plasma cholesterol in rats and humans using MIDA. Am J Physiol. 1993 Jan;264(1 Pt 1):E136-47.

14. Plōsch T, Kok T, Bloks VW, Smit MJ, Havinga R, Chimini G, et al. Increased hepatobiliary and fecal cholesterol excretion upon activation of the liver $\mathrm{X}$ receptor is independent of ABCA1.J Biol Chem. 2002 Sep 13;277(37):33870-7.

15. Bligh EG, Dyer WJ. A rapid method of total lipid extraction and purification. Can J Biochem Physiol. 1959 Aug;37(8):911-7.

16. Arca M, Montali A, Ciocca S, Angelico F, Cantafora A. An improved gas-liquid chromatographic method for the determination of fecal neutral sterols. J Lipid Res. 1983 Mar;24(3):332_ 5 .

17. Lee WN, Byerley LO, Bergner EA, Edmond J. Mass isotopomer analysis: theoretical and practical considerations. Biol Mass Spectrom. 1991 Aug;20(8):451-8. 
18. Hellerstein MK, Neese RA. Mass isotopomer distribution analysis: a technique for measuring biosynthesis and turnover of polymers. Am J Physiol. 1992 Nov;263(5 Pt 1):E988-1001.

19. Bandsma RH, Stellaard F, Vonk RJ, Nagel GT, Neese RA, Hellerstein MK, et al. Contribution of newly synthesized cholesterol to rat plasma and bile determined by mass isotopomer distribution analysis: bile-salt flux promotes secretion of newly synthesized cholesterol into bile. Biochem J. 1998 Feb 1;329 ( Pt 3:699-703.

20. Neese RA, Faix D, Kletke C, Wu K, Wang AC, Shackleton CH, et al. Measurement of endogenous synthesis of plasma cholesterol in rats and humans using MIDA. Am J Physiol Endocrinol Metab. 1993 Jan 1;264(1):E136-47.

21. Rensen PC, van Dijk MC, Havenaar EC, Bijsterbosch MK, Kruijt JK, van Berkel TJ. Selective liver targeting of antivirals by recombinant chylomicrons--a new therapeutic approach to hepatitis $\mathrm{B}$. Nat Med. 1995 Mar;1(3):221-5.

22. Mora A, Lipina C, Tronche F, Sutherland C, Alessi DR. Deficiency of PDK1 in liver results in glucose intolerance, impairment of insulin-regulated gene expression and liver failure. Biochem $\mathrm{J}$. 2005 Feb 1;385(Pt 3):639-48.

23. Sluimer JC, Kisters N, Cleutjens KB, Volger OL, Horrevoets AJ, van den Akker LH, et al. Dead or Alive: Gene expression profiles of advanced atherosclerotic plaques from autopsy and surgery. Physiol Genomics. 2007;30:335-41.

24. Ness GC, Chambers CM. Feedback and hormonal regulation of hepatic 3-hydroxy-3methylglutaryl coenzyme A reductase: the concept of cholesterol buffering capacity. Proc Soc Exp Biol Med. 2000 May;224(1):8-19.

25. Kosters A, Frijters RJJM, Schaap FG, Vink E, Plösch T, Ottenhoff R, et al. Relation between hepatic expression of ATP-binding cassette transporters G5 and G8 and biliary cholesterol secretion in mice. J Hepatol. 2003 Jun;38(6):710-6.

26. van der Velde AE, Brufau G, Groen AK. Transintestinal cholesterol efflux. Curr Opin Lipidol. 2010 Jun;21(3):167-71.

27. Schneider M, Van Geyte K, Fraisl P, Kiss J, Aragonés J, Mazzone M, et al. Loss or silencing of the PHD1 prolyl hydroxylase protects livers of mice against ischemia/reperfusion injury. Gastroenterology. 2010 Mar;138(3):1143-54.e1-2.

28. Gruber M, Hu C-J, Johnson RS, Brown EJ, Keith B, Simon MC. Acute postnatal ablation of Hif-2alpha results in anemia. Proc Natl Acad Sci U S A. 2007 Feb 13;104(7):2301-6.

29. Taylor AJ, Villines TC, Stanek EJ, Devine PJ, Griffen L, Miller M, et al. Extended-release niacin or ezetimibe and carotid intima-media thickness. N Engl J Med. 2009 Nov 26;361(22):2113-22. 30. Boden WE, Probstfield JL, Anderson T, Chaitman BR, Desvignes-Nickens P, Koprowicz $\mathrm{K}$, et al. Niacin in patients with low HDL cholesterol levels receiving intensive statin therapy. $\mathrm{N}$ Engl J Med. 2011 Dec 15;365(24):2255-67.

31. Bakris GL, Yu K-HP, Leong R, Shi W, Lee T, Saikali K, et al. Late-Breaking Orals. J Clin Hypertens. 2012 Jul 13;14(7):487-9.

32. Olson E, Demopoulos L, Haws TF, Hu E, Fang Z, Mahar KM, et al. Short-term treatment with a novel HIF-prolyl hydroxylase inhibitor (GSK1278863) failed to improve measures of performance in subjects with claudication-limited peripheral artery disease. Vasc Med. 2014 Dec $1 ; 19(6): 473-82$.

33. Higashijima Y, Tanaka T, Nangaku M. Structure-based drug design for hypoxia-inducible factor prolyl-hydroxylase inhibitors and its therapeutic potential for the treatment of erythropoiesisstimulating agent-resistant anemia: raising expectations for exploratory clinical trials. Expert Opin Drug Discov. 2013 Aug;8(8):965-76.

34. Rahtu-Korpela L, Karsikas S, Hörkkö S, Blanco Sequeiros R, Lammentausta E, Mäkelä KA, et al. HIF prolyl 4-hydroxylase-2 inhibition improves glucose and lipid metabolism and protects against obesity and metabolic dysfunction. Diabetes. 2014 Oct;63(10):3324-33. 
35. Takeda K, Ho VC, Takeda H, Duan LJ, Nagy A, Fong GH. Placental but not heart defects are associated with elevated hypoxia-inducible factor alpha levels in mice lacking prolyl hydroxylase domain protein 2. Mol Cell Biol. 2006;26(22):8336-46.

36. Franke K, Kalucka J, Mamlouk S, Singh RP, Muschter A, Weidemann A, et al. HIF-1 $\alpha$ is a protective factor in conditional PHD2-deficient mice suffering from severe HIF-2 $\alpha$-induced excessive erythropoiesis. Blood. American Society of Hematology; 2013 Feb 21;121(8):1436-45.

37. ClinicalTrials.gov. ClinicalTrials.gov - HIF-prolyl hydroxylase inhibitors in clinical trials [Internet]. 2015 [cited 2015 Jun 4]. Available from: https://clinicaltrials.gov/ct2/ results?term $=$ prolyl + hydroxylase + CKD\&Search $=$ Search

38. Holliger P, Prospero T, Winter G. "Diabodies": small bivalent and bispecific antibody fragments. Proc Natl Acad Sci U S A. 1993;90(14):6444-8.

39. Musallam KM, Porter JB, Sfeir PM, Tamim HM, Richards T, Lotta LA, et al. Raised haematocrit concentration and the risk of death and vascular complications after major surgery. $\mathrm{Br} \mathrm{J}$ Surg. 2013 Jul;100(8):1030-6.

40. Phrommintikul A, Haas SJ, Elsik M, Krum H. Mortality and target haemoglobin concentrations in anaemic patients with chronic kidney disease treated with erythropoietin: a metaanalysis. Lancet. Elsevier; 2007 Feb 3;369(9559):381-8.

41. Ramakrishnan SK, Taylor M, Qu A, Ahn S-H, Suresh M V, Raghavendran K, et al. Loss of von Hippel-Lindau protein (VHL) increases systemic cholesterol levels through targeting hypoxiainducible factor $2 \alpha$ and regulation of bile acid homeostasis. Mol Cell Biol. 2014 Apr;34(7):1208-20.

42. Drager LF, Li J, Shin M-K, Reinke C, Aggarwal NR, Jun JC, et al. Intermittent hypoxia inhibits clearance of triglyceride-rich lipoproteins and inactivates adipose lipoprotein lipase in a mouse model of sleep apnoea. Eur Heart J. 2012 Mar;33(6):783-90.

43. Robinson G V, Pepperell JCT, Segal HC, Davies RJO, Stradling JR. Circulating cardiovascular risk factors in obstructive sleep apnoea: data from randomised controlled trials. Thorax. 2004 Sep;59(9):777-82.

44. Drager LF, Bortolotto LA, Figueiredo AC, Krieger EM, Lorenzi GF. Effects of continuous positive airway pressure on early signs of atherosclerosis in obstructive sleep apnea. Am J Respir Crit Care Med. 2007 Oct 1;176(7):706-12.

45. Comondore VR, Cheema R, Fox J, Butt A, John Mancini GB, Fleetham JA, et al. The impact of CPAP on cardiovascular biomarkers in minimally symptomatic patients with obstructive sleep apnea: a pilot feasibility randomized crossover trial. Lung. Jan;187(1):17-22.

46. Coughlin SR, Mawdsley L, Mugarza JA, Wilding JPH, Calverley PMA. Cardiovascular and metabolic effects of CPAP in obese males with OSA. Eur Respir J. 2007 Apr;29(4):720-7.

47. Cummins EP, Berra E, Comerford KM, Ginouves A, Fitzgerald KT, Seeballuck F, et al. Prolyl hydroxylase-1 negatively regulates IkappaB kinase-beta, giving insight into hypoxia-induced NFkappaB activity. Proc Natl Acad Sci U S A. 2006;103(48):18154-9.

48. Wong MC, van Diepen JA, Hu L, Guigas B, de Boer HC, van Puijvelde GH, et al. Hepatocyte-specific IKK $\beta$ expression aggravates atherosclerosis development in APOE*3-Leiden mice. Atherosclerosis. 2012 Feb;220(2):362-8.

49. Sui Y, Park S-H, Xu J, Monette S, Helsley RN, Han S-S, et al. IKK $\beta$ links vascular inflammation to obesity and atherosclerosis. J Exp Med. 2014 May 5;211(5):869-86. 


\section{SUPPLEMENTAL DATA}

\section{Supplemental figure 1}

A
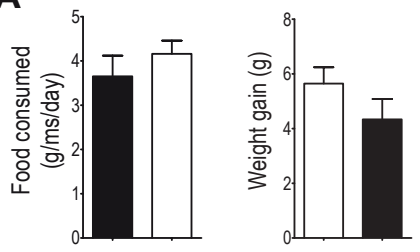

D

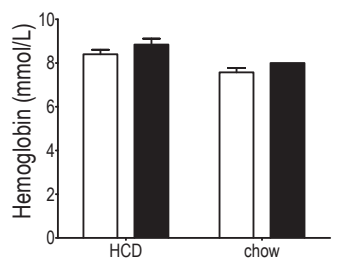

$\mathbf{F}$

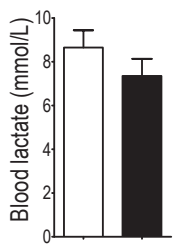

B
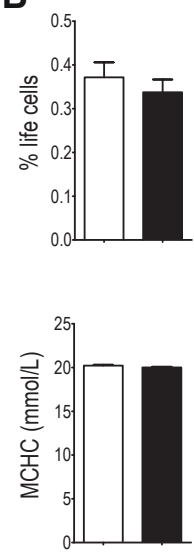

G

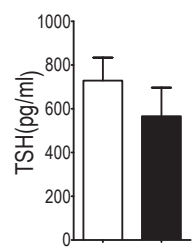

C

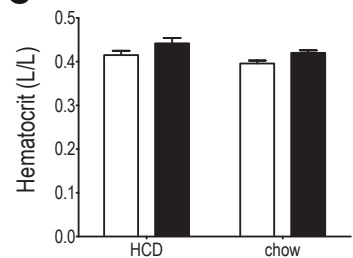

E

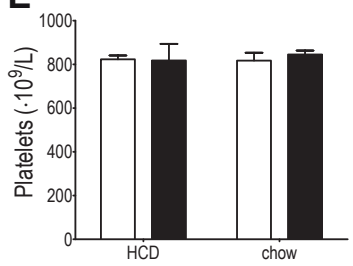

\section{Supplemental Figure 1.}

A. Food consumption and body weight gain upon 8 weeks of HCD of PHD1/LDLr/ and LDLr/ mice. B. Lineage-negative (lin-), Sca-1 ${ }^{+}, c$-Kit ${ }^{+}$cells (LSK), representing the hematopoietic stem cells isolated and quantified from bone marrow of PHD1- and WT mice. C. Hematocrit levels in whole blood of PHD1-LDLr ${ }^{-1}$ mice and controls on chow and after 8 weeks of HCD. D. Hemoglobin levels and mean cell hemoglobin concentration (MCHC) in both genotypes after 8 weeks of HCD. E. Platelet counts were measured both at chow and after 8 weeks of HCD. F. Lactate levels and $p H$ in whole blood in mice upon 8 weeks of HCD feeding. G. thyroid stimulating hormone (TSH) levels in plasma of PHD1/- LDL $r^{-/}$and controls after 8 weeks of HCD feeding. White bars represent $\mathrm{LDL} \mathrm{r}^{-/}$and black bars represent PHD1/-LDLr/- mice ( $n=10 /$ group). 


\section{Supplemental figure 2}

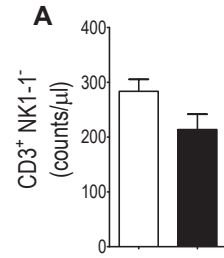

C
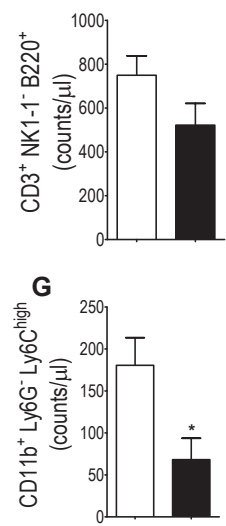

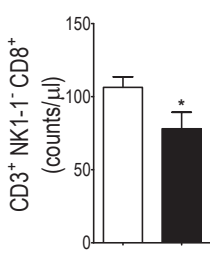

D
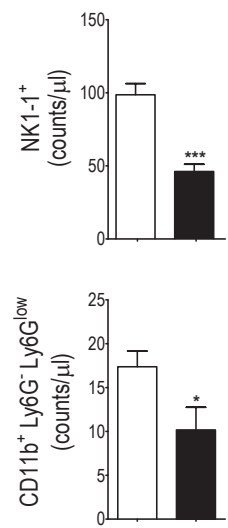

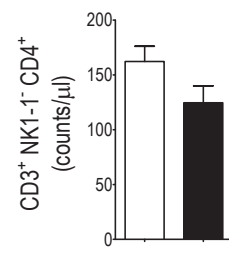

E
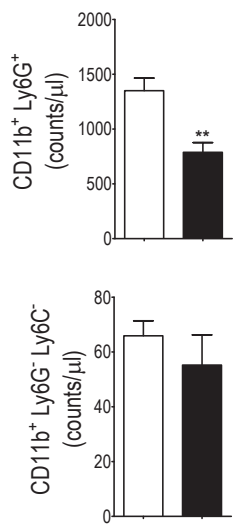

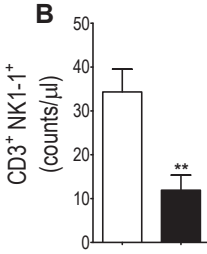

$\mathbf{F}$

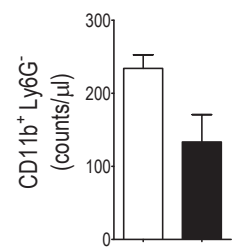

\section{Supplemental Figure 2.}

All graphs present quantitative flow cytometry in whole blood of PHD1- LDL $\mathrm{r}^{/-}$mice and controls on 8 weeles of HCD. A. Total T cells (CD3 ${ }^{+}$NK1-1), cytotoxic T cells (CD8) and T helper cells (CD4). B. NK-T cells gated as $\mathrm{CD}^{+}$and NK1-1 ${ }^{+}$. C. B cell, D. NK and E. Granulocyte count. F. Monocyte count and subpopulation count looking at G. Ly6Chigh (left panel), Ly6C low (middle panel) and Ly6C negative monocytes (right panel). White bars represent $L D L r^{\prime}$ and black bars represent PHD1/LDLr/- mice ( $n=10 /$ group). 
Supplemental figure 3
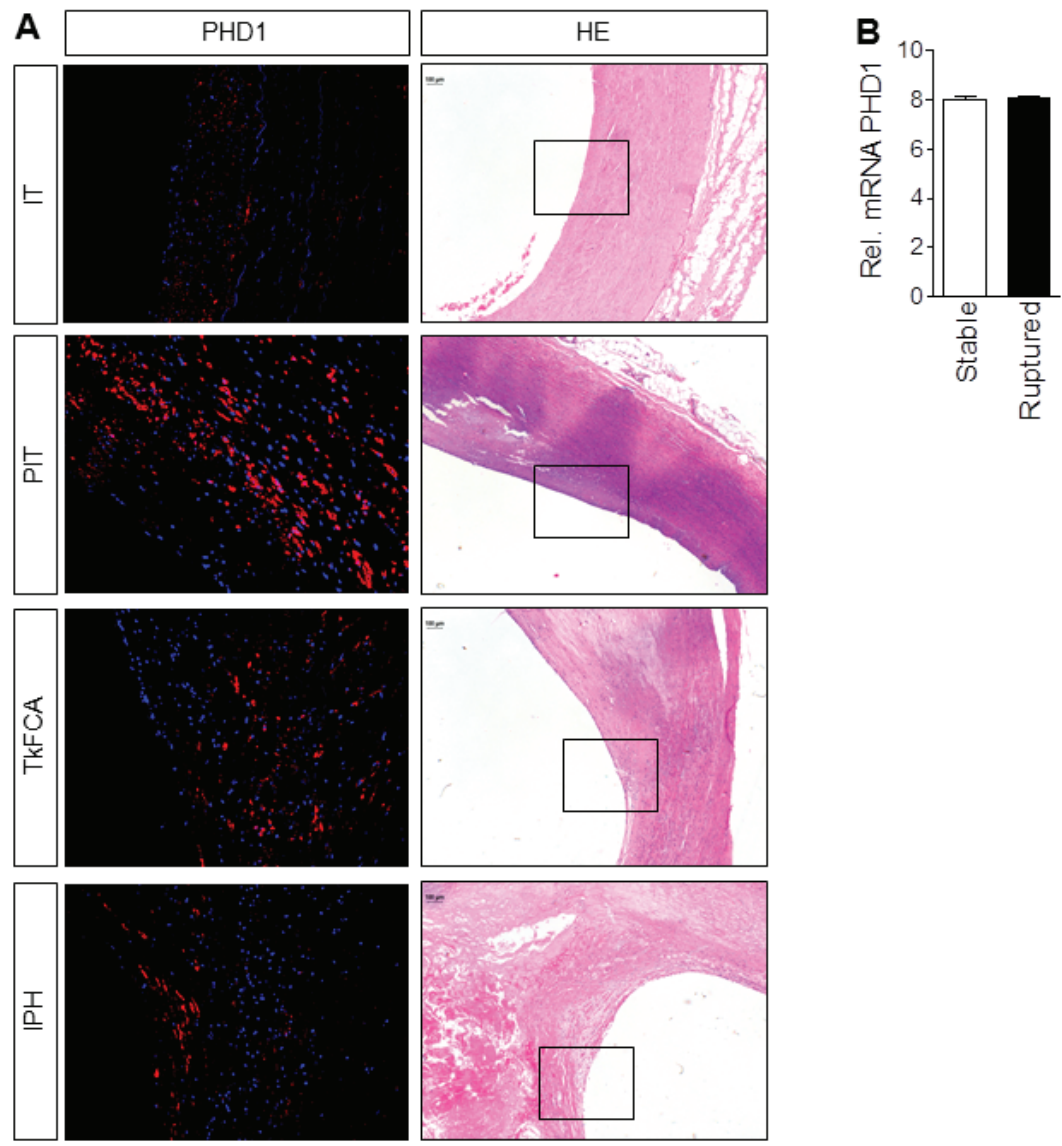

\section{Supplemental Figure 3.}

A. PHD1 expression (red) in different stages of buman atherosclerosis, HE for overview (samples from autopsy; IT - intimal thickening, PIT - pathological intimal thickening, TkFCA - Thick fibrous cap atheroma, IPH - intra-plaque haemorrbage). B. PHD1 $m \mathrm{RN} A$ expression (derived from microarrays) in stable and ruptures buman carotid endarterectomy plaques ( $n=21$ for stable and 23 for unstable). 


\section{Supplemental figure 4}

A
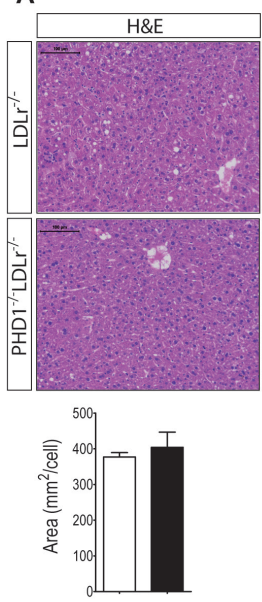

E

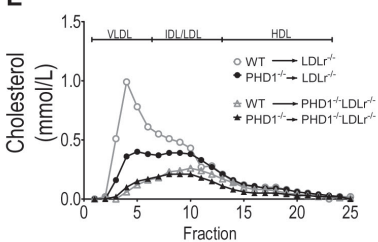

H

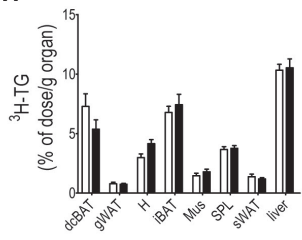

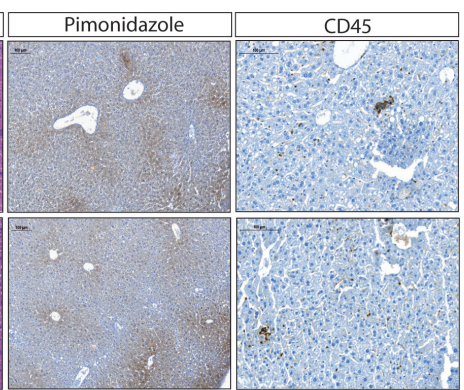
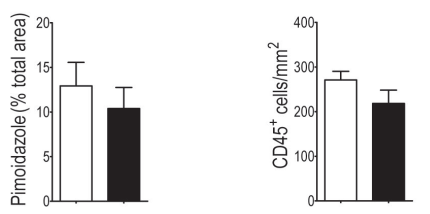

$\mathbf{F}$

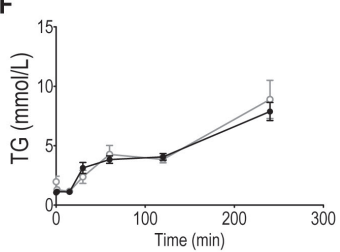

I

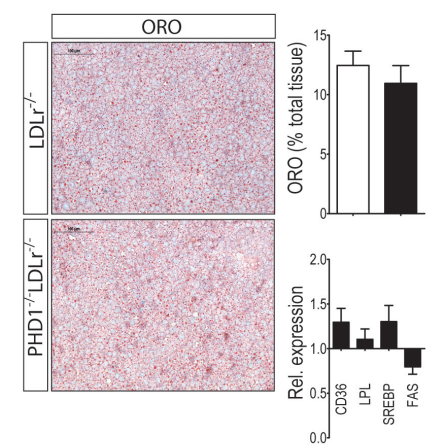

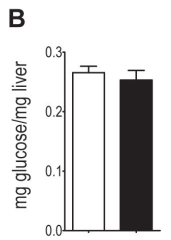
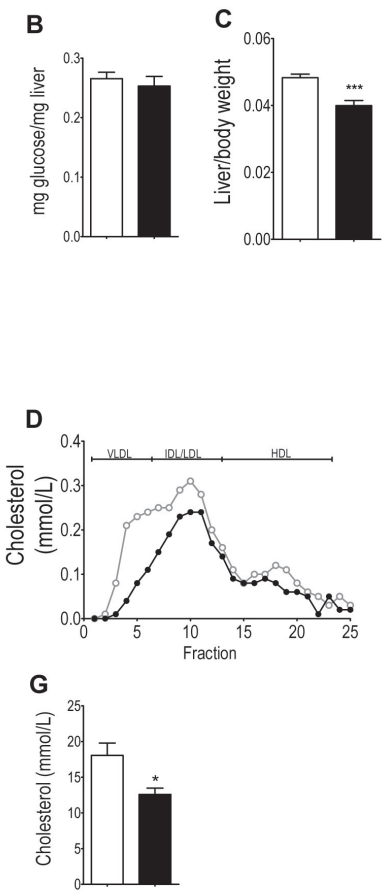

\section{Supplemental Figure 4.}

A. Representative pictures and quantifications of hepatocyte cell size (H\&E), hypoxia (pimonidazole) and inflammation (CD45) $\left(n=17 \mathrm{LDLr} /\right.$ and $\left.n=10 \mathrm{PHD}{ }^{-1}-\mathrm{LDLr} /-\right)$. B. Hepatic glycogen content corrected for hepatic protein content and C. Liver weights of PHD1/LDLr/- mice $(n=10)$ and LDLr/- controls $(n=17)$ after 8 weeks of HCD, corrected for body weight. D. Lipoprotein fractioning of plasma pools from $\mathrm{PHD}{ }^{-1} \mathrm{LDL} \mathrm{r}^{-/}$mice (white dots) and controls (black dots) on chow and E. in bone marrow transplanted mice after 8 weeks of $H C D(n=3 /$ pool $)$. Open symbols represent LDL $r^{*-}$ recipients with either WT or PHD1/- bone marrow and closed symbols show PHD1-LDLr/- recipients of either WT or PHD1\% bone marrow. F. TG accumulation in plasma of fasted mice upon triton WR1339 injection over time. G. Plasma cholesterol in HCD-fed mice prior to Poloxamer 407 injection. H. Accumulation of tritium-labelled 
$T G$ in a range of organs upon glycerol tri $\left[\begin{array}{l}\beta \\ H\end{array}\right]$ oleate-labeled emulsion particle injection. I. Representative pictures and quantification of Oil Red O stained livers, including relative expression of hepatic CD36, LPL, SREBP and FAS expression in PHD1-LDL $r^{-1}$ mice, relative to LDL $r^{\prime-}$ mice ( $n=10 /$ group). If not differently indicated, white bars represent $L D L r^{\circ}$ and black bars represent $P H D 1 / L D L r^{r /-}$ mice $(n=10 /$ group $)$.

Supplemental figure 5
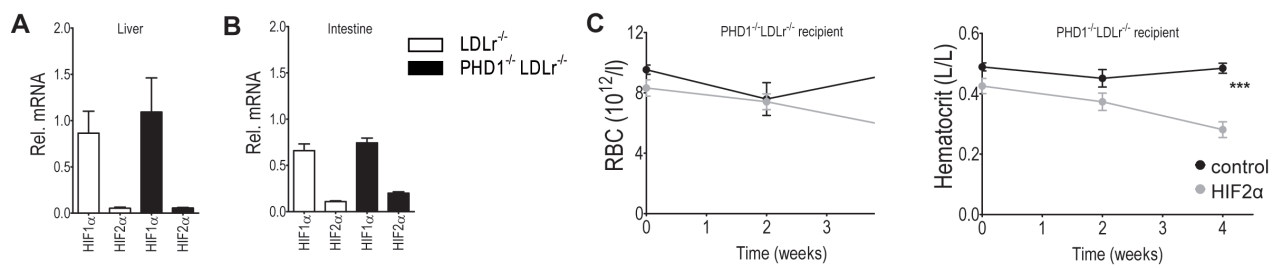

Supplemental Figure 5 .

A. Specificity and knockdown confirmation (qPCR) in livers and B. intestines of HIF-2a ASO in LDLr/- mice (white bars) and PHD1-LDLr/- (black bars). C. Red blood cell (left graph) and hematocrit (right graph) changes upon HIF-2a ASO injections (grey lines) in PHD1/-LDLr/- recipients. All graphs represent $n=9 /$ group.

\begin{tabular}{|lll|}
\hline & Regression coefficient & p-value \\
\hline Plaque size & 0.7550 & 0.4502 \\
\hline Lipid Core & 195867443307917.0000 & T 0.0501 \\
\hline Haemorrhage size & 15834969044486.0000 & 0.1133 \\
\hline Haemorrhage tissue ratio & 0.5410 & 0.5885 \\
\hline Necrotic core size & 0.7107 & 0.4772 \\
\hline Necrotic core tissue ratio & 105968450749331.0000 & 0.2893 \\
\hline CD31 vessel count & -0.0612 & 0.9512 \\
\hline CD31 \%staining & -0.3805 & 0.7036 \\
\hline CD68 cell count & 0.4115 & 0.6807 \\
\hline CD68 tissue ratio & -0.8658 & 0.3866 \\
\hline T cells cell count & 0.2266 & 0.8208 \\
\hline T cell tissue ratio & -0.5262 & 0.5987 \\
\hline
\end{tabular}

Supplemental Table 1:

Correlation of PHD1 expression in human plaques with plaque phenotype. 


\begin{tabular}{|c|c|c|}
\hline Gene & Forward primer & Reverse primer \\
\hline $18 S$ & TGC ATG GCC GTT CTT AGT TG & AGT TAG CAT GCC AGA GTC TCG TT \\
\hline GAPDH & CAA CTC ACT CAA GAT TGT CAG CAA & TGG CAG TGA TGG CAT GGA \\
\hline Cyclophillin & CAA ATG CTG GAC CAA ACA CAA & TTC ACC TTC CCA AAG ACC ACA T \\
\hline CD36 & GCC AAG CTA TTG CGA CAT GA & AAA AGA ATC TCA ATG TCC GAG ACT TT \\
\hline LPL & TTT GTG AAA TGC CAT GAC AAG & CAG ATG CTT TCT TCT CTT GTT TGT \\
\hline SREBP2 & ATG AGG CTA AGA GCA GCG CAA & TCA CAG CAC ACA GAG CCA TGT \\
\hline FAS & TGC TCC CAG CTG CAG GC & GCC CGG TAG CTC TGG GTG TA \\
\hline ABCG5 & TCC TGC ATG TGT CCT ACA GC & ATT TGC CTG TCC CAC TTC TG \\
\hline ABCG8 & ATC CAT TGG CCA CCC TTG T & GCG TCT GTC GAT GCT GGT C \\
\hline LRP1 & GGA CCA CCA TCG TGG AAA & TCC CAG CCA CG TGA TAG \\
\hline AMPK $\alpha$ & GTC GAC GTA GCT CCA AGA CC & ATC GTT TTC CAG TCC CTG TG \\
\hline SR-B1 & GCA AGA AGC CAA GCT ATA GGG & AAG AAG CGG GGT GTA GGG \\
\hline FXR & CAA AAT GAC TCA GGA GGA GTA CG & TCC TTG ATG TAT TGT CTG TCT GG \\
\hline NPC1L1 & CAA CAT CTT CAT CTT TCT TCT TGA G & GCC AAT GTG AGC CTC TCG \\
\hline ACAT2 & ATT CCA GCC ATA AAG CAA GC & TTT AGC TAT TGC CGC AGA CA \\
\hline ASBT & GAC TAG CTG GTC AAC CCT GGT A & GGG GGA GAA GGA GAG CTG TA \\
\hline FXR & CAA AAT GAC TCA GGA GGA GTA CG & TCC TTG ATG TAT TGT CTG TCT GG \\
\hline PCSK-9 & TGG AAC CTG GAG CGA ATT AT & CCT GGC TGC TTC CAT CAG \\
\hline ABCA1 & CCC AGA GCA AAA AGC GAC TC & GGT CAT CAT CAC TTT GGT CCT TG \\
\hline PPAR $\alpha$ & ATG CCA GTA CTG CCG TTT TC & GGCCTTGACCTTGTTCATGT \\
\hline PPARY & AAG AGC TGA CCC AAT GGT TG & ACC CTT GCA TCC TTC ACA AG \\
\hline Prox1 & CGA CAT CTC ACC TTA TTC AGG A & TTG CCT TTT TCA AGT GAT TGG \\
\hline HMG-CoA & TTG CTG TGA GAA TGT GAT CGG & ACA AGA CAG CCT TCC GTC GTT \\
\hline
\end{tabular}

Supplemental Table 2:

Primer sequences (murine). 


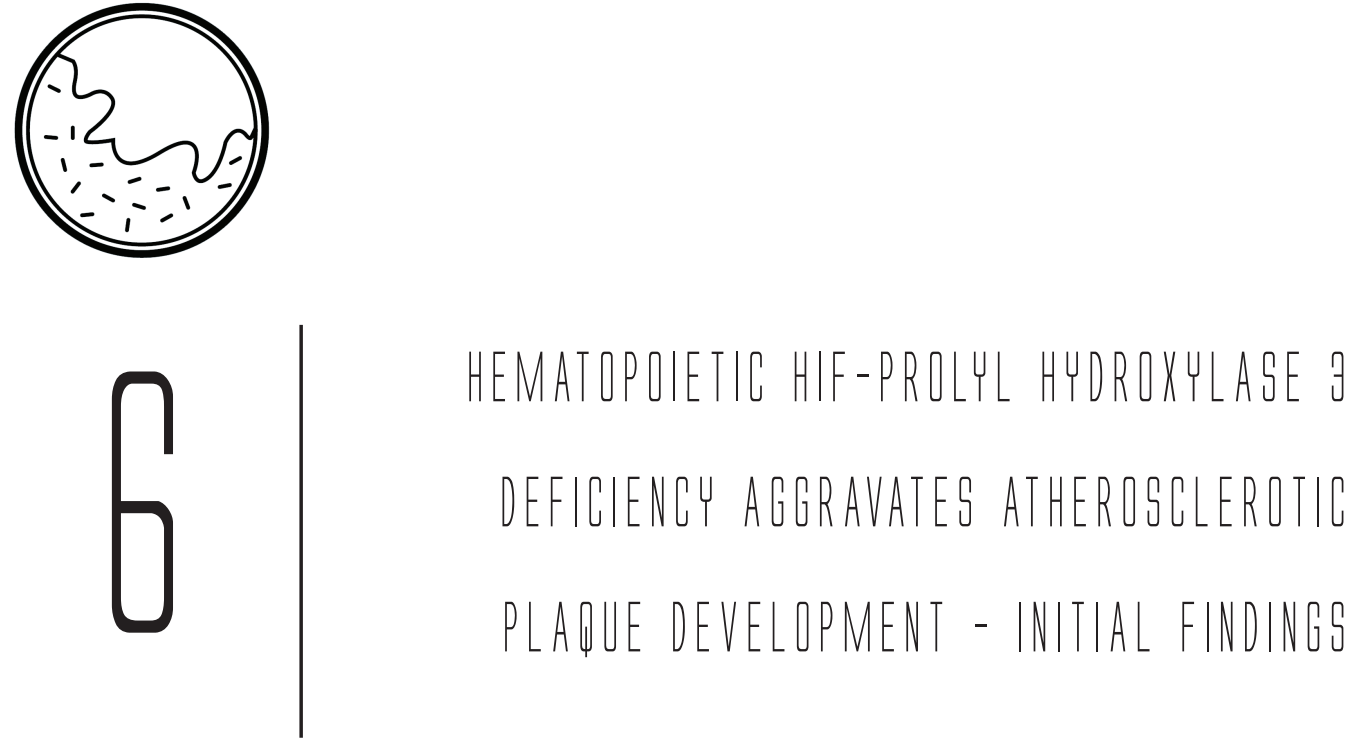

margch e, demandt jaf, theelen tl, tullemans bme, hefFron S, Jangsen a, Rousch mum, figher ea, carmeliet pC, heeneman S, Blegsen eal, daemen maap, glumer dC IN PREPARATION. 



\section{ABSTRACT}

\section{BACKGROUND}

Atherosclerotic plaque hypoxia co-localizes with inflammation in humans and mice. As alternative to inflammation targeting, we propose to interfere with hypoxic signaling in atherosclerotic plaques. Expression of the oxygen sensor Hypoxia-inducible factorprolyl hydroxylase 3 (PHD 3) is induced in pro-inflammatory macrophages, prompting us to study the effect of hematopoietic PHD3 deficiency compared to whole body PHD3 deficiency in atherosclerosis development.

\section{APPROACH AND RESULTS}

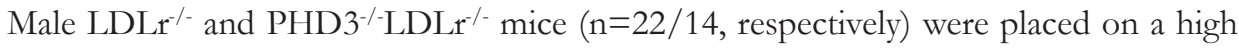
cholesterol diet $\left(0.25 \%\right.$ cholesterol, HCD) for 10 weeks. In a separate study, male LDLr ${ }^{-/}$ mice ( $n=18 / 16$, respectively) were transplanted with wild-type or PHD3 ${ }^{-/}$bone marrow and placed on HCD for 8 weeks. All mice were injected with pimonidazole $(25 \mathrm{mg} / \mathrm{kg}$ intraperitoneally) 1 hour prior to sacrifice for subsequent tissue hypoxia analysis. Aortic root plaque and necrotic core size (H\&E) was not altered in whole body $\mathrm{PHD}^{-/} \mathrm{LDLr}^{-/}$ mice, despite a small elevation in plasma cholesterol levels $(+11 \%)$. Blood hematocrit levels were enhanced in whole body $\mathrm{PHD}^{-/} \mathrm{LDLr}^{-/}$mice. In contrast, hematopoietic $\mathrm{PHD}^{-/}$did not affect plasma cholesterol levels but enhanced plaque size (40\%) and necrotic core expansion (50\%), likely attributable to enhanced intra-plaque apoptosis (twofold increase, TUNEL). Apoptotic cell clearance capacity (efferocytosis) was not affected by hematopoietic PHD3 ${ }^{-}$in situ and in vitro, suggesting that apoptosis itself was elevated in hematopoietic $\mathrm{PHD}^{-/}$mice. Plaque collagen (Sirius Red), smooth muscle cell ( $\alpha$ SMA) and macrophage content (MAC3) and adventital microvessel density (CD31) remained unchanged. Additionally, hematopoietic PHD3 deficiency increased circulating granulocyte numbers as well as a splenic and lymph node cDCs. Erythropoietic parameters in blood were not altered by hematopoietic PHD3-- mice.

\section{CONCLUSION}

Whole body PHD3 deficiency slightly elevated plasma cholesterol levels, without affecting atherosclerosis. On the other hand, hematopoietic PHD3 deficiency aggravated early atherosclerotic plaque development, likely by enhancing apoptosis. 


\section{INTRODUCTION}

Recent therapeutic approaches aim at reducing inflammation in atherosclerosis, by inhibiting inflammatory mediator biosynthesis and/or signaling, or by inducing immune tolerance by means of vaccination. Multiple agents are undergoing clinical trials at the moment (reviewed in (1)). However, results are partially disappointing, when investigating cardiovascular outcome (stroke, myocardial infarction and cardiovascular mortality incidence) (1), suggesting that other factors are likely involved. In human and murine atherosclerotic plaques, inflammatory areas co-localize with hypoxia and hypoxiainducible factor (HIF) expression (2,3). Also, inflammation in human plaques, measured by labelled $\left[{ }^{18} \mathrm{~F}\right]$-fluorodeoxyglucose uptake via positron emission tomography (PET) correlated with hypoxia (4). Thus, instead of targeting inflammation, we propose to interfere with hypoxic signaling in atherosclerotic plaques, in particular in macrophages, thereby potentially also affecting inflammation.

On a cellular level, hypoxia is mainly sensed by HIF-prolyl hydroxylases (PHD) 1, 2 and 3. In the presence of oxygen, PHDs hydroxylate HIF- $\alpha$ subunits, thereby targeting them for degradation. In hypoxia, however, $\mathrm{HIF}-\alpha$ subunits are stabilized, heterodimerize with $\beta$ subunits and subsequently transcriptionally regulate genes involved in cellular metabolism, growth and proliferation, angiogenesis and apoptosis.

We recently showed reduced atherosclerosis development and plasma cholesterol levels in $\mathrm{PHD}^{-/} \mathrm{LDLr}^{-/}$mice (chapter 5). A similar phenotype was observed for PHD2 hypomorphism and upon general PHD inhibition in mice (5). In turn, macrophagespecific PHD2 deficiency resulted in enhanced macrophage collagen production and enhanced plaque development (Theelen, Marsch et al. in preparation). Thus, PHDs seemed to cell type-specifically regulate atherosclerosis development.

As opposed to PHD1 and PHD2, PHD3 is actually upregulated in hypoxic cardiac myocytes, smooth muscle cells and endothelial cells (6-8). In accordance with the colocalization of hypoxia and inflammation in the plaque, human pro-inflammatory (M1) macrophages also showed enhanced PHD3 expression (9). On the other hand, PHD3 deficient peritoneal macrophages showed enhanced pro-inflammatory activation upon LPS stimulation as compared to controls. Next to that, PHD3 plays a role in regulation of apoptosis, where PHD3 deficiency seems to protect cardiomyocytes, neurons and macrophages from apoptosis under normoxic conditions (10-12). In turn, PHD3 deficiency enhances apoptosis in neutrophils under hypoxic conditions (13), suggesting a cell type-specific or oxygen-dependent role of PHD3 in apoptosis regulation. 
In this study, we study the effect of $\mathrm{PHD}^{-/}$in stromal and hematopoietic cells on atherosclerotic plaque development. We hypothesize that PHD3 deficiency negatively affects macrophage function and results in enhanced apoptosis in the hypoxic plaque environment.

\section{MATERIAL AND METHODS}

\section{ANIMALS}

All mouse experiments were approved by the regulatory authority of Maastricht University Medical Centre and performed in compliance with the guidelines described in the directive 2010/63/EU of the European Parliament. All mice were bred at least 9x on C57/JBl6 background and male LDLr/- mice were obtained from an in-house breeding colony, originating from Charles River (Wilmington, MA, USA) and refreshed every 10 generations to avoid genetic drift. All animals were housed in individually ventilated cages (GM500, Techniplast) in groups of up to 5 animals per cage, with bedding (corncob, Technilab-BMI) and cage enrichment. Cages were changed weekly, reducing handling of the mice to one handling per week during non-intervention periods.

\section{ATHEROSCLEROSIS MODELS}

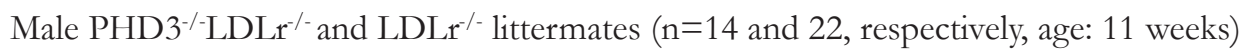
were fed a high cholesterol diet (HCD) ad libitum (0.25\% cholesterol, SDS 824171) for 10 weeks. For bone marrow transplantations, $\mathrm{LDLr}^{-/-}$recipients $(\mathrm{n}=18 / 16)$ were placed on antibiotic water, containing neomycin $(100 \mathrm{mg} / \mathrm{L}$; Gibco, Carlsbad, CA, USA) and polymyxin B sulphate $(60.000 \mathrm{U} / \mathrm{L}$; Gibco) for 7 weeks in total while being fed a chow diet. After one week of antibiotic water drinking, bone marrow was isolated from female wild-type (WT; C57J/B16) and PHD3 ${ }^{-/-}$mice and transplanted (1x107 cells/mouse) into irradiated $\mathrm{LDLr}^{-/-}$recipients $(2 \times 6 \mathrm{~Gy}, \mathrm{n}=18 / 16)$. Mice were left to recover for 6 weeks on chow diet and subsequently placed on HCD for 8 weeks.

\section{atherosclerogIS QUANTIFICATION AND IMMUNOHISTOCHEMISTRY}

One hour prior to sacrifice, all mice were intraperitoneally (i.p.) injected with the hypoxiaspecific marker pimonidazole (100 mg/kg, hypoxyprobe Omni HP3 kit, Hypoxyprobe Inc. Burlington, MA, USA). Mice were euthanized with a pentobarbital overdose $(100 \mathrm{mg} / \mathrm{kg}$ i.p.) and blood was withdrawn via the right ventricle for flow cytometry, absolute white and red blood cell counts (Coulter Ac.T diff, Beckman Coulter) and 
total cholesterol analysis. Mice were perfused via the left cardiac ventricle with PBS containing sodium nitroprusside ( $0.1 \mathrm{mg} / \mathrm{ml}$; Sigma-Aldrich, Seelze, Germany).

Aortic root and all organs were subsequently excised and fixed in 1\% PFA overnight, processed and paraffin-embedded. Aortic roots and arches were serially sectioned and stained with hematoxylin and eosin (H\&E, Sigma) for plaque area and lipid core content quantification. Five consecutive H\&E sections at $20 \mu \mathrm{m}$ intervals were analyzed blindly using computerized morphometry (Leica QWin V3, Cambridge, UK) and averaged per mouse. Sections within this $100 \mu \mathrm{m}$ interval were used for remaining immunohistochemical stainings. If appropriate, antigen retrieval was performed at pH 6 (Dako REAL target retrieval, Dako). Atherosclerotic plaques were characterized for macrophage content $\left(\mathrm{MAC}^{+}\right.$area/total area, BD Cat. No. 553322), smooth muscle cell content $\left(\alpha \mathrm{SMA}^{+}\right.$area/total tissue, $\alpha \mathrm{SMA}^{+}$area; $\alpha \mathrm{SMA}$ Sigma F3777), adventitial microvessel density (number of $\mathrm{CD} 31^{+}$microvessels/adventitial area, BD Cat. No. 550274) and collagen (Picosirius Red $^{+}$area/plaque area, Polyscience 09400). Hypoxia was detected in the aortic roots and livers using a rabbit polyclonal antibody (clone 2627) directed against pimonidazole derivates, formed in vivo specifically in hypoxic but living cells (\% pimonidazole/total plaque area). Apoptosis (TUNEL ${ }^{+}$nuclei/plaque area, Roche) and clearance of apoptotic cells (efferocytosis) in situ were analyzed in entire tissue sections using TUNEL ${ }^{+} / \mathrm{MAC}^{+}$double immunohistochemistry as the ratio of bound/free apoptotic cells according to established methods $(14,15)$. Leucocyte count was quantified in the liver as $\mathrm{CD}^{+} 5^{+}$cells (rat-anti-mouse CD45 LCA ly-5 antibody, clone 30F11 B\&D) per liver area.

\section{total and hepatic cholegterol and trIglycerIdes}

Plasma was separated by centrifugation, and stored at $-80^{\circ} \mathrm{C}$ until further use. Standard enzymatic techniques were used to assess plasma cholesterol (cholesterol FS'10; Ref: 113009910 021; Diagnostic Systems GmbH, Holzheim, Germany) and plasma triglycerides (TG) (FS5' Ecoline REF 157609990314; DiaSys - Diagnostic Systems GmbH, Holzheim, Germany) automated on the Cobas Fara centrifugal analyzer (Roche).

For hepatic cholesterol and TG content, livers were homogenated in SET buffer $(250 \mathrm{mM}$ sucrose, 2mM EDTA 10mM Tris, pH6.8). Upon two freeze-thaw cycles and suction of the homogenate through an insulin syringe, TG and cholesterol were measured in the homogenates using the kits described above. Cholesterol and TG levels were corrected for protein content assessed in the same homogenate using a BCA kit (Thermo Fisher Scientific, Cat. No. 23227). 


\section{FLOW CYTOMETRY AND BLOOD PARAMETERS}

Cells isolated from whole blood were analyzed using flow cytometry ( $\mathrm{n}=10$ per group) Spleens and lymph nodes were dissociated into single-cell suspensions, and enzymatically digested for dendritic cell separation using liberase and DNAse (both $0.2 \mathrm{mg} / \mathrm{ml}$, Roche) for 30 minutes in RPMI medium. Blood and spleen were subjected to erythrocyte lysis. The following specific antibodies were used to detect leucocyte subsets: leucocytes (CD45 ${ }^{+}$, Biolegend), T cells (CD3 $\varepsilon^{+}$, NK1-1; Miltenyi, eBioscience, resp.), T helper cells $\left(\mathrm{CD} 4^{+}, \mathrm{BD}\right)$, cytotoxic $\mathrm{T}$ cells $\left(\mathrm{CD} 8 \mathrm{a}^{+}, \mathrm{BD}\right)$, effector $\mathrm{T}$ cells $\left(\mathrm{CD} 44^{\text {high }}, \mathrm{CD} 62^{\text {low }}\right.$ BD, eBioscience, resp.), central memory $\mathrm{T}$ cells $\left(\mathrm{CD} 44^{\text {high }}, \mathrm{CD} 66^{\text {high }}\right)$, naïve $\mathrm{T}$ cells $\left(\mathrm{CD} 44^{\text {low }}\right.$,

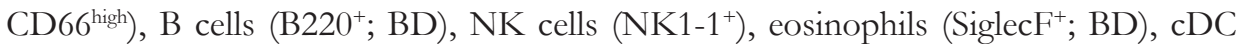
(CD11chigh, eBioscience), pDC (CD11 $\left.\mathrm{c}^{\mathrm{mid}}, \mathrm{B} 220^{+}, \mathrm{Ly} 6 \mathrm{C}^{+}\right)$, granulocytes (CD11b $\mathrm{b}^{\text {high }}$ Ly $6 G^{\text {high, }}$ BD, eBioscience, resp.) and monocytes $\left(C D 11 b^{\text {high }}\right.$ Ly6G ${ }^{\text {low }}$ Ly6C $C^{\text {high/intermediate/low; }}$ Miltenyi). Data were acquired using a FACS Canto II and analyzed with FACSdiva software (BD).

For erythropoietic parameter analysis, whole blood was diluted 1:10 in Hepes buffer, $\mathrm{pH}$ 7.45 (10mM Hepes, $136 \mathrm{mM} \mathrm{NaCl}, 2.7 \mathrm{mM} \mathrm{KCl}, 2 \mathrm{mM} \mathrm{MgCl}_{2}, 0.1 \%$ glucose, $0.1 \%$ BSA) and subsequently measured on the XP3000 Sysmex analyzer (Sysmex, Chuo-ku Kobe, Japan).

\section{IN VITRO CELL CULTURE AND EFFEROCYTOSIS}

Bone marrow was isolated and cells were cultured for seven days in RPMI-1640 (Gibco with Glutamax, 2g/L glucose) supplemented with 10\% FCS, 100U/ml PenicillinStreptomycin, and 15\% L929-conditioned medium to generate bone marrow-derived macrophages (BMDM). For efferocytosis analysis in vitro, Jurkat T cells were labeled with calcein-AM $(1 \mu \mathrm{g} / \mathrm{ml}$ Invitrogen) prior to induction of apoptosis by UV irradiation (15 min 254nm, UVS-26, 6W bulb 0.02J/s/ $\mathrm{cm}^{2}$ ) and added 3:1 to BMDM. Macrophages were exposed to $21 \%, 1 \%$ or $0.2 \% \mathrm{O}_{2}$ during 45 minutes of efferocytosis (Invivo2 1000, Ruskinn technology LTD, Pencoed, UK). After thorough washing, macrophages were dissociated and analyzed using flow cytometry for the percentage of calcein/jurkat ${ }^{+}$ macrophages.

\section{COLLAGEN SYNTHESIS}

Macrophage collagen synthesis was measured after $72 \mathrm{~h}$ with the last $48 \mathrm{~h}$ under hypoxia $\left(0.2 \% \mathrm{O}_{2}\right)$, in 24 wells plates with a density of $0.4 \times 10^{6}$ cells per well. Collagen was visualized by Sirius Red staining ( $1 \%$ Sirius red in $0.01 \mathrm{M} \mathrm{HCl}$ for $1 \mathrm{~h}$ at $\mathrm{RT}$ ), after fixation with 
$3.7 \%$ formaldehyde for $1 \mathrm{~h}$ at RT. The amount of collagen was spectrophotometrically determined in cell lysates at $544 \mathrm{~nm}$.

\section{Collagen degradation}

The functional activity of matrix metalloproteinase (MMPs) was determined using OmniMMPтM fluorogenic substrate (Enzo Life Science, BML-P126-0001). The substrate with the sequence Mca-Pro-Leu-Gly-Leu-Dpa-Ala-Arg-NH2 incorporates a quencher (Dpa) and a fluorescent side (Mca). Upon MMP cleavage of the quencher fluorescence can be detected. A total of $0.14 * 10^{6}$ BMDM (lysed in PBS containing 1\% Trition-X100) was incubated with $180 \mathrm{mM} \mathrm{CaCl}_{2} 65 \mu \mathrm{M}$ OmniMMP substrate at $37^{\circ} \mathrm{C}$ and the fluorescence was detected at an interval of two minutes on a Spectromax (Ex 328nm, Em 393nm, Molecular Devices SPECTRAmax M2). The slope of fluorescence intensity increase was subsequently analyzed.

\section{STATISTICAL ANALYSIS}

All data are presented as mean \pm SEM. All parameters were analyzed using independent sample tests and were tested for normal distribution using Shapiro-Wilk normality test. Parameters with two groups were compared with student's t-test or Mann-Whitney ranksum test. A p-value of $\mathrm{p}<0.05$ was considered significant $(* \mathrm{p}<0.05$, ** $\mathrm{p}<0.01, * * *$ $\mathrm{p}<0.001)$. 


\section{RESULTS}

hematopoletic phoz deficiency enhances plaque development, While WhOLE bODY PHD-1-LDLR ${ }^{-1}$ DOES NOT AFFECT ATHEROSCLEROIS

Atherosclerotic plaque development was investigated in hematopoietic PHD3-1and whole body $\mathrm{PHD}^{-/} \mathrm{LDLr}^{-/}$mice. Hematopoietic $\mathrm{PHD}^{-/}$mice presented with enhanced plaque and necrotic core size compared to their wild-type (WT) controls (Figure 1A). This effect was not observed in whole body PHD3 $3^{-/} \mathrm{LDLr}^{-/}$mice (Figure 1B). Plaque hypoxia or macrophage content were not affected in hematopoietic or whole body PHD3-1-LDLr ${ }^{-1}$ (Figure 1C-D), suggesting that enhanced macrophage influx or proliferation or plaque hypxoxia does not underlie enhanced plaque size in the hematopoietic $\mathrm{PHD}^{-/}$model. In general, plaques of hematopoietic PHD3/- mice were much smaller than whole body $\mathrm{PHD}^{--} \mathrm{LDLr}^{-/}$plaques (Figure $1 \mathrm{~A}, \mathrm{~B}$ ). This could be due to the duration of diet ( 8 weeks instead of 10 in the whole body $\mathrm{PHD}^{-/} \mathrm{LDLr}^{-/}$), in addition irradiation affects plaque development (16). 
Figure 1
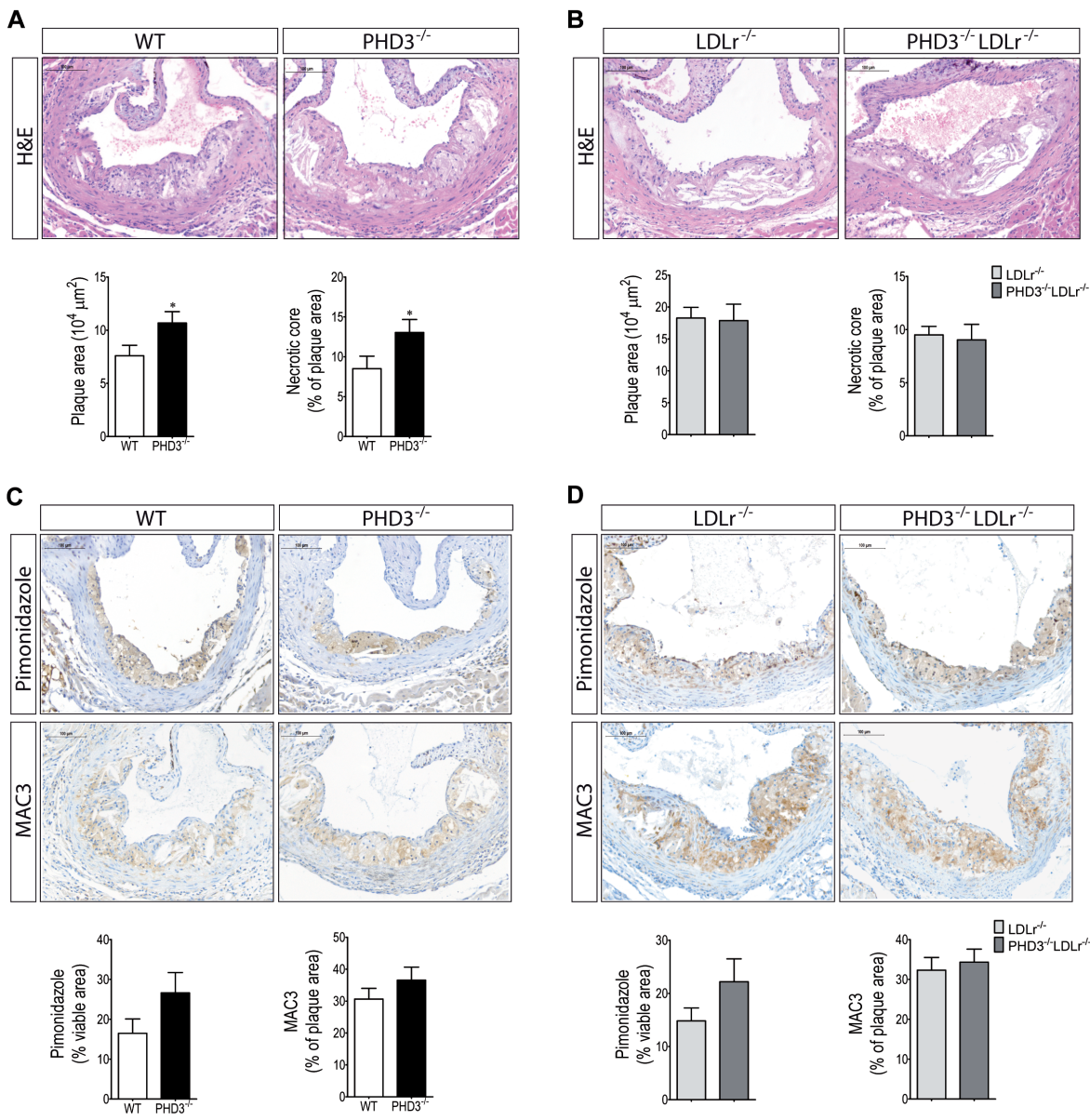

Figure 1: Hematopoietic PHD3 $\%$ increases atherosclerotic plaque and necrotic core size.

Representative pictures and quantification of aortic root plaque area and necrotic core content (\% plaque area) of A. hematopoietic PHD ${ }^{3-}$ and controls and B. whole body PHD ${ }^{-1-L D L r^{-}}$. C. Aortic root pimonidazole and MAC3 content in hematopoietic PHD ${ }^{-1}$ as well as D. whole body PHD ${ }^{-1} \mathrm{LDLr}^{\circ}$. 
Plasma cholegterol leVels are elevated IN WhOLE bODY PhD马 DEFICIENT MICE ONLY

Neither cholesterol nor TG levels were altered in hematopoietic PHD3 ${ }^{-/}$mice (Figure 2A). Also, hematopoietic PHD3־ did not affect body weight or organ weights. Prior to bone marrow transplantations, recipient $\mathrm{LDLr}^{-/}$mice were matched for body weight (Figure 2C). Hepatic liver weight, hepatocyte size, hepatic inflammation and hypoxia, hepatic cholesterol, triglyceride and glycogen content were unaltered in hematopoietic PHD3-- (Supplemental Figure 1).

In contrast to the hematopoietic $\mathrm{PHD}^{-/}$, whole body $\mathrm{PHD}^{-/} \mathrm{LDLr}^{-/}$showed slightly enhanced plasma cholesterol and TG levels upon high cholesterol diet (Figure 2B). Whole body $\mathrm{PHD}^{-/} \mathrm{LDLr}^{-/}$mice were heavier, although weight gain was similar, and showed enhanced liver weight, even when corrected for the elevated body weight

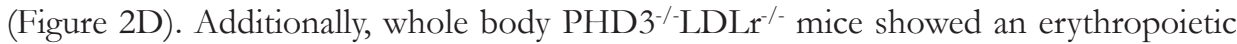
effect, while hematopoietic $\mathrm{PHD}^{-/}$mice presented with a similar hematocrit compared to their controls (Figures 2E-H).

Figure 2

A
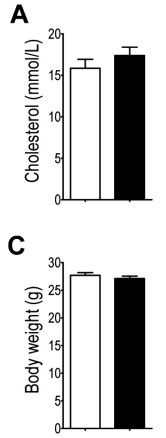

E
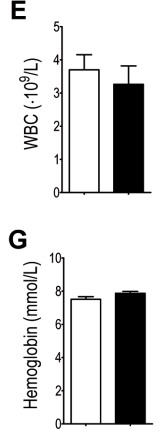
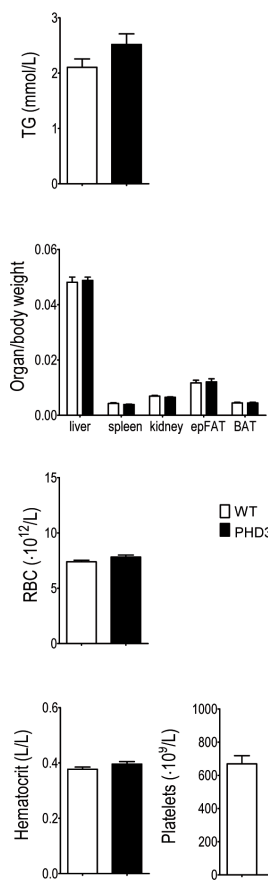

$\mathrm{WT}$
$\mathrm{PHD}^{-1}$

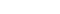

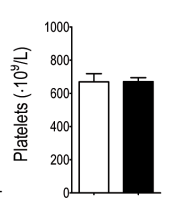
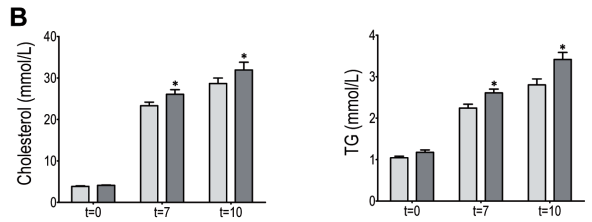

D
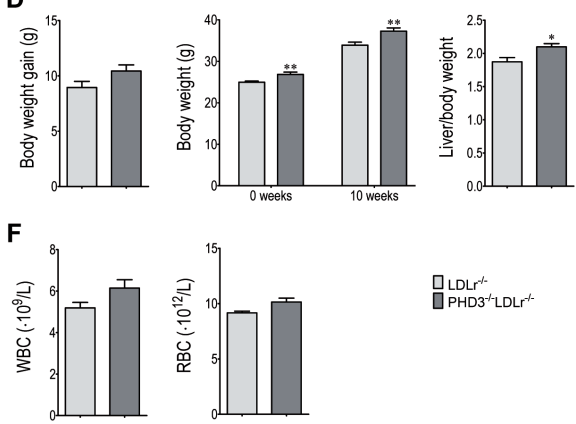

H
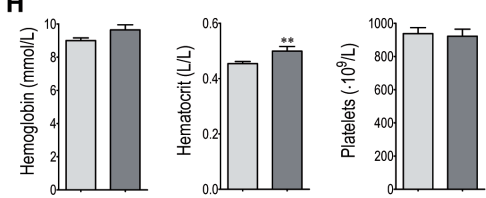

Figure 2: Whole body $\mathrm{PHD3}^{-/}$enhanced body and liver weight upon high cholesterol diet. 


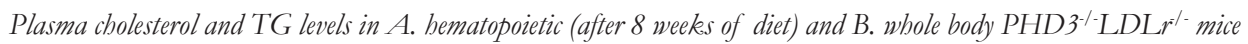
compared to their respective controls (prior to start of HCD, $t=0$; after 7 weeks, $t=7$ and 10 weeks, $t=10$ ). C. Body

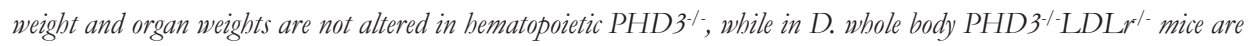
heavier with heavier livers compared to their LDL $r^{\prime}$ - controls. E. White blood cell, red blood cell count in hematopoietic PHD ${ }^{-/}$and F. whole body PHD ${ }^{--} \mathrm{LDLr}^{-/}$were unchanged. G. hemoglobin, hematocrit and platelet levels in hematopoietic PHD $3^{-1}$ and H. whole body PHD ${ }^{-1-L D L r / .}$.

\section{hematopoletic phog-1- afFected circulating granulocytes and tigsue cocs}

Next, we investigated leucocyte distribution in blood of hematopoietic $\mathrm{PHD}^{-/-}$(Figure $3 \mathrm{~A}, \mathrm{C}$ ) and whole body $\mathrm{PHD}^{-/} \mathrm{LDLr}^{-/}$mice (Figure 3 B,D). None of the subsets studied were affected numerically in either model, except from an induction in $\mathrm{Ly} 6 \mathrm{G}^{+}$granulocyte numbers in the hematopoietic PHD3/- (Figure 3A). In lymph node and spleen, an increase in conventional dendritic cells (cDCs) was observed in the hematopoietic $\mathrm{PHD}^{-/-}$model (Figure 3E).

Figure 3

A
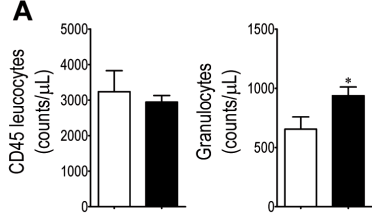

QWT

C
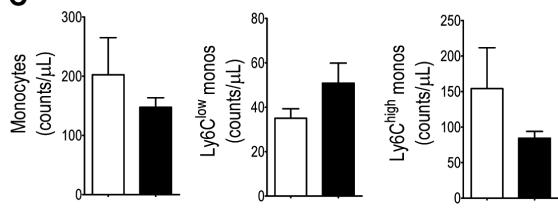

B
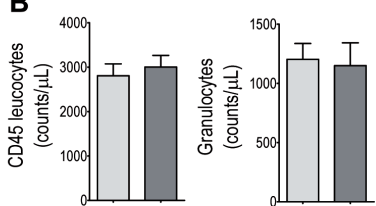

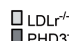

PHD3'LDLr't

D
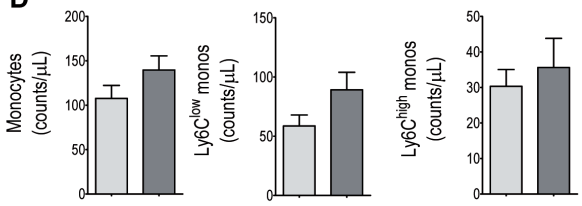

E

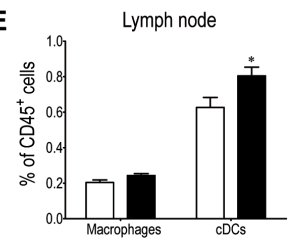

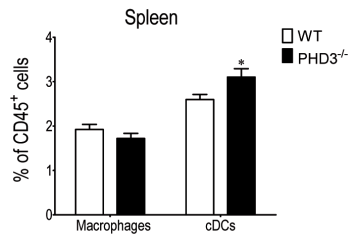

Figure 3: Leucocyte subsets in blood, spleen and lymph nodes.

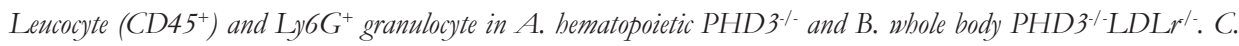
Ly6GCD11 b bong mocytes and Ly6C Cow and Ly6C high monocytes on hematopoietic PHD ${ }^{-1}$ and D. whole body $\mathrm{PHD}^{3-\mathrm{LDLr}}$. E. CD11 $c^{\text {bigh }} \mathrm{cDC}$ numbers in lymph nodes and spleen of hematopoietic PHD ${ }^{-\%}$ mice. 
HEMATOPOIETIC PHDZ DEFICIENCY DID NOT AFFECT ADVENTITIAL MICROVESSEL DENSITY OR PLAQUE COLLAGEN AND SMOOTH MUSCLE CELL CONTENT BUt INCREASE PLAQUE APOPTOSIS

No microvessels were detected in atherosclerotic plaques of hematopoietic or whole body PHD 3 deficiency and controls. In the plaque adventitia, CD31 positive microvessel density was unchanged in hematopoietic PHD ${ }^{\%}$ mice (Figure 4A). Additionally, hematopoietic $\mathrm{PHD}^{-} \%$ did not induce differences in plaque smooth muscle cell or collagen content in the plaque (Figure 4A). Hematopoietic PHD3 deficiency enhanced apoptosis, compared to their WT controls (Figure 4C), while efferocytosis analyzed in situ (ratio of bound/unbound apoptotic cells to $\mathrm{MAC}^{+}$macrophages) was unchanged (Figure 4B). Thus enhanced apoptosis might underlie the necrotic core expansion and accompanying plaque growth seen in these mice, however, the cell type undergoing apoptosis in hematopoietic $\mathrm{PHD}^{\%}$ remains unknown. Also in vitro, PHD3\% macrophages did not show any differences in collagen synthesis or MMP activity, as a marker of collagen degradation capacity (Figure 4C). Additionally, macrophage PHD3 deficiency did not alter apoptotic $\mathrm{T}$ cell clearance in vitro, supporting the in vivo data (Figure 4D).

Figure 4

A
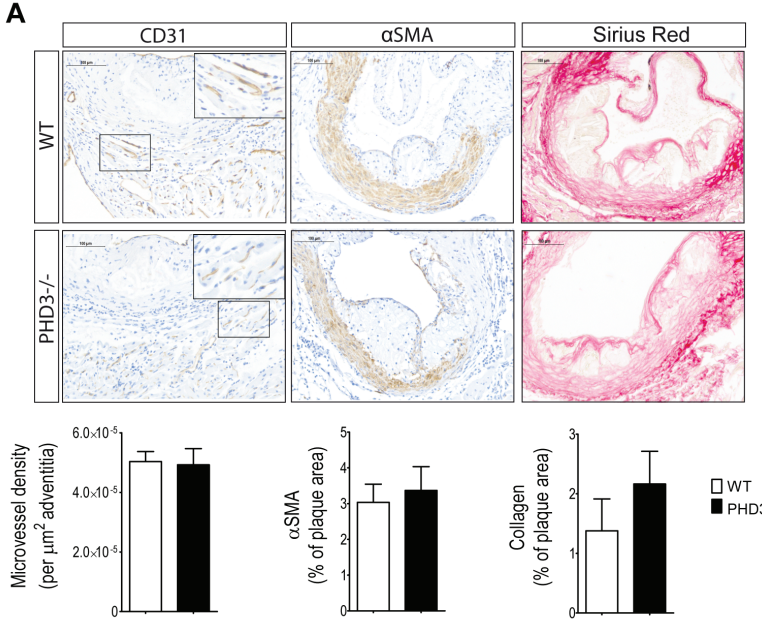

C

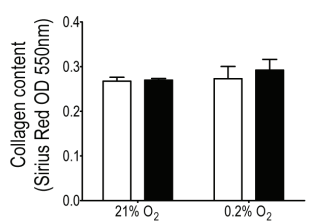

B
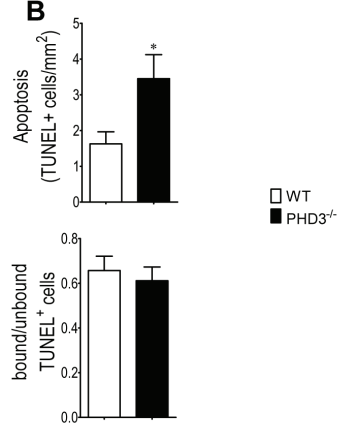

Figure 4: Enhanced apoptosis in hematopoietic PHD3 deficient plaques. 
A. Representative pictures and quantification of microvessel density (CD31), smooth muscle cell content (aSMA) and collagen deposition (Sirius Red) in the aortic roots of hematopoietic PHD $3 \%$ mice and controls. B. Apoptosis (TUNEL) and efferocytosis (bound/unbound TUNEL ${ }^{+}$cells to $M A C 3^{+}$macrophages) in situ in plaques of bematopoietic PHD $3 \%$ and control mice. C. In vitro collagen content and MMP activity assay (analyzed as increase in fluorescence upon successful MMP-mediated quencher cleavage over time) of PHD3-1 macrophages and controls. D. Efferocytosis of apoptotic Jurkat cells by PHD3 deficient macrophages. Significance indicated presents effects relative to $21 \%$ oxygen conditions of the same genotype.

\section{DISCUSSION}

This study investigated the effect of the absence of the oxygen sensor PHD3 on atherosclerosis development in mice. We showed that hematopoietic PHD3 ${ }^{-/}$enhanced plaque progression, necrotic core size and apoptosis within the plaque. As efferocytosis was unchanged, enhanced apoptosis might result in the necrotic core expansion and accompanying plaque growth seen in these mice. In comparison, whole body $\mathrm{PHD}^{-/} \mathrm{LDLr}^{-/}$did not affect plaque progression and development, despite slightly elevated plasma cholesterol levels.

PHD3 has mainly been studied in ischemia reperfusion injury, where PHD3 deficiency reduced infarct size by inducing angiogenesis and increasing capillary density $(17,18)$. In our murine model of atherosclerosis, we did not observe any angiogenesis effects. We only investigated microvessel formation in the adventitia, as atherosclerotic plaques did not show any vessel formation. However, studying angiogenesis in murine atherosclerosis has proven difficult (19) and thus a lack of angiogenesis in our murine model does not exclude an angiogenic effect on human atherosclerosis in a translational approach.

Independent of plaque angiogenesis, we showed enhanced apoptosis in hematopoietic $\mathrm{PHD}^{\%}$ mice potentially underlying the enhanced necrotic core size and plaque expansion. However, the cell type undergoing apoptosis remains unknown. Nevertheless, enhanced apoptosis in PHD3 deficiency is partially in line with current literature, showing enhanced neutrophil apoptosis under hypoxic conditions in PHD3 deficient mice (12,13). In contrast to hypoxic neutrophils, normoxic sympathetic neurons from PHD3 lacking mice showed reduced apoptosis in a HIF-2 $\alpha$-dependent manner. In addition, PHD3 deficient mice presented with increased superior cervical ganglia numbers, though they were not functional and hypoxia was not assessed (11). In line with that, PHD3 silencing by siRNA in vitro protected normoxic rat cardiomyocytes from doxorubicin-induced apoptosis (10). Thus, PHD3 seems to differentially regulate apoptosis in normoxic and hypoxic conditions and enhances apoptosis in hypoxic neutrophils, but not in other normoxic neurons and cardiomyocytes. Here, we show 
enhanced apoptosis in hematopoietic PHD3 deficient hypoxic atherosclerotic plaques.

A mechanistic link between PHD3 and apoptosis has been demonstrated for the Bax/Bcl-2 complex. PHD3 overexpression induced apoptosis in normoxic cardiomyocytes by inhibiting the formation of the anti-apoptotic protein complex $\mathrm{Bax} / \mathrm{Bcl}-2$ (10). Indeed, pro-apoptotic Bax expression in smooth muscle cells and macrophages within human plaques has been associated with enhanced apoptosis (20) and a reduced $\mathrm{Bcl}-2 / \mathrm{Bax}$ ratio was observed in apoptotic cells of advanced human atherosclerotic plaques (21). However, whether the $\mathrm{Bax} / \mathrm{Bcl}-2$ complex is altered in our model of hematopoietic PHD3 deficiency remains to be established.

Intriguingly, we do not observe any difference in plaque and necrotic core size, hypoxia or macrophage content in the whole body $\mathrm{PHD}^{-/} \mathrm{LDLr}^{-/}$model. We have to note that plaques were less advanced in the hematopoietic $\mathrm{PHD}^{-/-}$model as compared to the whole body $\mathrm{PHD}^{-/} \mathrm{LDLr}^{-/}$. Thus, one could speculate that enhanced apoptosis also occurs in the whole body $\mathrm{PHD}^{-/}$, which has been compensated for upon further progression of the plaque. However, this remains to be shown by for example investigating early stage whole body $\mathrm{PHD}^{-/-}$plaque development. On the other hand, stromal PHD3 or smooth muscle cell PHD3 may compensate its hematopoietic effects. For instance, alleviated smooth muscle cell apoptosis - in line with reduced apoptosis in cardiomyocytes (10) - or paracrine survival signaling may counterbalance pro-apoptotic signaling in hematopoietic $\mathrm{PHD}^{-/}$cells.

PHD3 whole body deficiency slightly increased plasma cholesterol level and body and liver weights. Nevertheless, this rather small increase in plasma cholesterol levels was either not sufficient to affect atherosclerotic plaque growth in whole body PHD $3^{-}$ $\mathrm{LDLr}^{-/}$mice, or was overruled by other yet unknown anti-atherosclerotic effects of

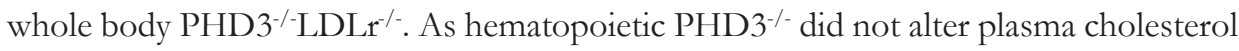
levels, the cholesterol effect is likely mediated by stromal cells, in line with a stromal effect of PHD1 on cholesterol metabolism (chapter 5). Plasma cholesterol levels might be close to saturation in the high cholesterol diet fed $\mathrm{PHD}^{-/} \mathrm{LDLr}^{-/}$mice, potentially limiting the effect size seen in whole body PHD3 deficiency mice after 10 weeks of high cholesterol diet. Despite this, atherosclerosis development was not affected in these mice. This is in contrast to our recent data on PHD1 deficiency resulting in reduced plasma cholesterol levels and reduced plaque size (chapter 5). Also, PHD2 hypomorphism and inhibition was recently shown to reduce plasma cholesterol levels, at least on chow diet, and to decrease atherosclerotic plaque progression $(5,22)$. $\mathrm{PHD}^{-} /$has been shown to result in profound HIF-2 $\alpha$ stabilization in the liver (23). In turn, hepatic HIF-2 $\alpha$ was crucial for 
hepatic cholesterol accumulation and elevation in plasma cholesterol levels in van Hippel Lindau deficient mice (24). Hepatic HIF-2 $\alpha$ stabilization and cholesterol accumulation remains to be investigated in whole body $\mathrm{PHD}^{-/}$mice. Silencing HIF-2 $\alpha$ in PHD3 deficient mice would reveal a potential causal relationship for cholesterol elevation in PHD3 deficiency. Nevertheless, general PHD inhibitors, partially inhibiting PHD3, reduce plasma cholesterol in humans $(25,26)$. This suggests that PHD3 is not the crucial PHD with respect to cholesterol homeostasis in general PHD inhibition.

These general PHD inhibitors are undergoing clinical trials as erythropoiesis-stimulating agents to treat anemia patients. Whole body PHD3 deficiency slightly increased hematocrit levels, though not to the same extent as observed for general PHD inhibition $(25,26)$. Thus, PHD3 might contribute but does not seem to be essential for PHD inhibition-mediated erythropoiesis.

\section{CONCLUSIONS}

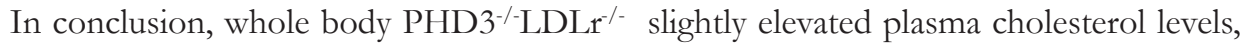
an effect that is likely mediated by stromal cells, as hematopoietic PHD $3^{-/}$did not affect cholesterol levels. Despite this cholesterol-raising effect, whole body PHD3 ${ }^{-/} \mathrm{LDLr}^{-/}$ mice did not show any effects on plaque development. Rather, hematopoietic $\mathrm{PHD}^{\%}$ aggravated early atherosclerotic plaque development, probably by inducing intra-plaque apoptosis. 


\section{FUNDING}

VENI fellowship of the Dutch Organization for Scientific Research (to J.C.S. 016.116.017); PhD-student Fellowship from the Cardiovascular Research Institute Maastricht (to T.L.T.) 


\section{REFERENCES}

1. Bäck M, Hansson GK. Anti-inflammatory therapies for atherosclerosis. Nat Rev Cardiol. Nature Publishing Group, a division of Macmillan Publishers Limited. All Rights Reserved.; 2015 Apr;12(4):199-211.

2. Sluimer JC, Gasc J-M, van Wanroij JL, Kisters N, Groeneweg M, Sollewijn Gelpke MD, et al. Hypoxia, hypoxia-inducible transcription factor, and macrophages in human atherosclerotic plaques are correlated with intraplaque angiogenesis. J Am Coll Cardiol. 2008 Apr 1;51(13):1258-65.

3. Marsch E, Theelen TL, Demandt JAF, Jeurissen M, van Gink M, Verjans R, et al. Reversal of Hypoxia in Murine Atherosclerosis Prevents Necrotic Core Expansion by Enhancing Efferocytosis. Arterioscler Thromb Vasc Biol. 2014 Sep 25;34(12):2545-53.

4. van der Valk FM, Sluimer JC, Vöö SA, Verberne HJ, Nederveen AJ, Windhorst AD, et al. In Vivo Imaging of Hypoxia in Atherosclerotic Plaques in Humans. JACC Cardiovasc Imaging. 2015 Nov 5;8(11):1340-1.

5. Rahtu-Korpela L, Määttä J, Dimova EY, Hörkkö S, Gylling H, Walkinshaw G, et al. HypoxiaInducible Factor-Prolyl 4-Hydroxylase-2 Inhibition Protects Against Development of Atherosclerosis. Arterioscler Thromb Vasc Biol. 2016 Feb 4;ATVBAHA.115.307136 - .

6. Cioffi CL, Liu XQ, Kosinski PA, Garay M, Bowen BR. Differential regulation of HIF1 alpha prolyl-4-hydroxylase genes by hypoxia in human cardiovascular cells. Biochem Biophys Res Commun. 2003 Apr 11;303(3):947-53.

7. Pescador N, Cuevas Y, Naranjo S, Alcaide M, Villar D, Landázuri MO, et al. Identification of a functional hypoxia-responsive element that regulates the expression of the egl nine homologue 3 (egln3/phd3) gene. Biochem J. 2005 Aug 15;390(Pt 1):189-97.

8. Metzen E, Stiehl DP, Doege K, Marxsen JH, Hellwig-Bürgel T, Jelkmann W. Regulation of the prolyl hydroxylase domain protein 2 (phd2/egln-1) gene: identification of a functional hypoxiaresponsive element. Biochem J. 2005 May 1;387(Pt 3):711-7.

9. Escribese MM, Sierra-Filardi E, Nieto C, Samaniego R, Sánchez-Torres C, Matsuyama T, et al. The prolyl hydroxylase PHD3 identifies proinflammatory macrophages and its expression is regulated by activin A. J Immunol. 2012 Aug 15;189(4):1946-54.

10. Liu Y, Huo Z, Yan B, Lin X, Zhou Z-N, Liang X, et al. Prolyl hydroxylase 3 interacts with Bcl-2 to regulate doxorubicin-induced apoptosis in H9c2 cells. Biochem Biophys Res Commun. 2010 Oct 15;401(2):231-7.

11. Bishop T, Gallagher D, Pascual A, Lygate CA, de Bono JP, Nicholls LG, et al. Abnormal Sympathoadrenal Development and Systemic Hypotension in PHD3-/- Mice. Mol Cell Biol. 2008 Mar 10;28(10):3386-400.

12. Swain L, Wottawa M, Hillemann A, Beneke A, Odagiri H, Terada K, et al. Prolyl-4hydroxylase domain 3 (PHD3) is a critical terminator for cell survival of macrophages under stress conditions. J Leukoc Biol. 2014 Sep;96(3):365-75.

13. Walmsley SR, Chilvers ER, Thompson AA, Vaughan K, Marriott HM, Parker LC, et al. Prolyl hydroxylase 3 (PHD3) is essential for hypoxic regulation of neutrophilic inflammation in humans and mice. J Clin Invest. 2011 Mar;121(3):1053-63.

14. Schrijvers DM, De Meyer GRY, Kockx MM, Herman AG, Martinet W. Phagocytosis of apoptotic cells by macrophages is impaired in atherosclerosis. Arterioscler Thromb Vasc Biol. 2005 Jun;25(6):1256-61.

15. Thorp E, Cui D, Schrijvers DM, Kuriakose G, Tabas I. Mertk receptor mutation reduces efferocytosis efficiency and promotes apoptotic cell accumulation and plaque necrosis in atherosclerotic lesions of apoe-/- mice. Arterioscler Thromb Vasc Biol. 2008 Aug;28(8):1421-8.

16. Stewart FA, Heeneman S, Te Poele J, Kruse J, Russell NS, Gijbels M, et al. Ionizing radiation accelerates the development of atherosclerotic lesions in ApoE-/- mice and predisposes to an 
inflammatory plaque phenotype prone to hemorrhage. Am J Pathol. 2006 Feb;168(2):649-58.

17. Oriowo B, Thirunavukkarasu M, Selvaraju V, Adluri RS, Zhan L, Takeda K, et al. Targeted gene deletion of prolyl hydroxylase domain protein 3 triggers angiogenesis and preserves cardiac function by stabilizing hypoxia inducible factor 1 alpha following myocardial infarction. Curr Pharm Des. 2014 Jan;20(9):1305-10.

18. Loinard C, Ginouvès A, Vilar J, Cochain C, Zouggari Y, Recalde A, et al. Inhibition of prolyl hydroxylase domain proteins promotes therapeutic revascularization. Circulation. 2009 Jul 7;120(1):50 9 .

19. Khurana R, Simons M, Martin JF, Zachary IC. Role of angiogenesis in cardiovascular disease: a critical appraisal. Circulation. 2005 Sep 20;112(12):1813-24.

20. Kockx MM, De Meyer GRY, Muhring J, Jacob W, Bult H, Herman AG. Apoptosis and Related Proteins in Different Stages of Human Atherosclerotic Plaques. Circulation. 1998 Jun 16;97(23):2307-15.

21. Saxena A, McMeekin JD, Thomson DJ. Expression of Bcl-x, Bcl-2, Bax, and Bak in endarterectomy and atherectomy specimens. J Pathol. 2002 Mar;196(3):335-42.

22. Rahtu-Korpela L, Karsikas S, Hörkkö S, Blanco Sequeiros R, Lammentausta E, Mäkelä KA, et al. HIF prolyl 4-hydroxylase-2 inhibition improves glucose and lipid metabolism and protects against obesity and metabolic dysfunction. Diabetes. 2014 Oct;63(10):3324-33.

23. Groen AK, Bloks VW, Verkade H, Kuipers F. Cross-talk between liver and intestine in control of cholesterol and energy homeostasis. Mol Aspects Med. 2014 Jun;37:77-88.

24. Ramakrishnan SK, Taylor M, Qu A, Ahn S-H, Suresh M V, Raghavendran K, et al. Loss of von Hippel-Lindau protein (VHL) increases systemic cholesterol levels through targeting hypoxiainducible factor $2 \alpha$ and regulation of bile acid homeostasis. Mol Cell Biol. 2014 Apr;34(7):1208-20.

25. Bakris GL, Yu K-HP, Leong R, Shi W, Lee T, Saikali K, et al. Late-Breaking Orals. J Clin Hypertens. 2012 Jul 13;14(7):487-9.

26. Olson E, Demopoulos L, Haws TF, Hu E, Fang Z, Mahar KM, et al. Short-term treatment with a novel HIF-prolyl hydroxylase inhibitor (GSK1278863) failed to improve measures of performance in subjects with claudication-limited peripheral artery disease. Vasc Med. 2014 Dec $1 ; 19(6): 473-82$. 


\section{SUPPLEMENTAL DATA}

\section{Supplemental Figure 1}

A
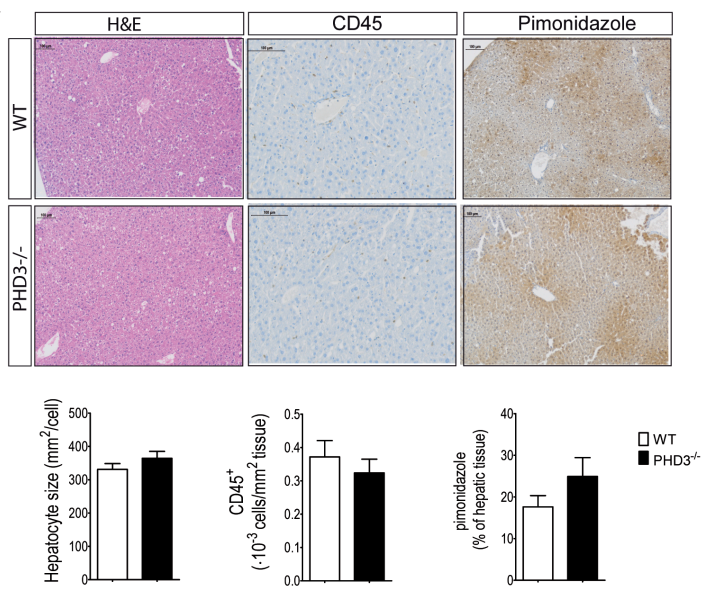
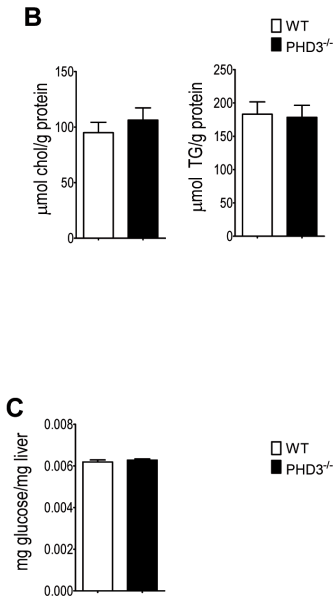

Supplemental Figure 1: No differences in liver morphology in hematopoietic PHD3-/- mice compared to their wild-type controls.

A. Representative pictures and quantification of bepatocyte size, inflammatory infux (CD45) and bypoxia (pimonidazole). B. Neither hepatic cholesterol, triglyceride nor C. glycogen contents are changed in hematopoietic KO mice. 




$$
\begin{aligned}
& 2 \\
& 71 \\
&
\end{aligned}
$$





\section{MAIN FINDINGS OF THE DISSERTATION}

The overall aim of this disseration was to explore the causative role of plaque hypoxia in atherosclerotic disease development and progression and to establish the therapeutic potential of interfering with plaque oxygenation.

\section{The essential results of the work presented in this dissertation, which are discussed in this chapter, are:}

1. Atherosclerotic plaques are hypoxic from initiation on and hypoxia mainly co-localizes with plaque macrophages, pointing towards enhanced oxygen consumption rather than reduced oxygen diffusion to underlie plaque hypoxia (chapter 3).

2. Enhancing systemic oxygen supply using carbogen gas reverses plaque hypoxia and alleviate plaque burden (chapter 3).

3. Prolonged dietary nitrate did not affect plaque hypoxia or burden, and no conclusions on the role of oxygen consumption in atherosclerosis can be drawn from this study (chapter 4).

4. Reducing whole body cellular oxygen consumption in PHD1 deficient mice can reduce plaque hypoxia and atherosclerotic plaque progression; however cholesterol-lowering effect in PHD1 deficiency trumps plaque hypoxia effects. In turn, PHD3 deficiency did not affect plaque hypoxia, not allowing for solid conclusions on the causal contribution of cellular oxygen consumption to plaque progression (chapter 5,6).

5. Hematopoietic PHD3 deficiency enhances plaque apoptosis and plaque growth, while hematopoietic PHD1 deficiency does not alter plaque development (chapter 5,6).

6. PHD1 deficiency enhances non-biliary cholesterol efflux directly from plasma to the feces, thereby reducing VLDL and LDL-contained cholesterol by $20-40 \%$ (chapter 5). In turn, PHD3 deficiency slightly increases plasma cholesterol levels (chapter 6). 


\section{ENHANCING SYSTEMIC OXYGEN AVAILABILITY: OXYGEN thERAPY FOR ATHEROSCLEROSIS}

The human body adjusts to systemic hypoxia via carotid body chemoreceptors, pulmonary artery smooth muscle cells (both rapid) and erythropoietin producing cells (slow) (1). On the tissue level, oxygen tensions differ between organs (2). We and others could show pimonidazole positivity even under physiological conditions, e.g. in the entire intestinal epithelial cell lining (3), as well as in the renal medulla, and venous areas of the liver (chapter 3, (4)). Pimonidazole forms adducts with thiol groups in proteins, peptides and amino acids in case of hypoxia $\left(\mathrm{pO}_{2}<10 \mathrm{mmHg}\right.$, less than $1 \%$ oxygen). Subsequent adducts can be detected immunohistochemically (5). Thus, pimonidazole represents an "on/off” signal of oxygen tensions below or above 1\%. However, also within one organ oxygen tensions can vary. This is particularly obvious for human adipose tissue, where oxygen levels actually range from $\sim 3$ and $11 \% \mathrm{O}_{2}(\sim 23-84 \mathrm{mmHg})(6,7)$. Indeed, in rabbit atherosclerotic plaques, oxygen tensions of 0 to $15 \mathrm{mmHg}$ were recorded close to the media of atherosclerotic arteries compared with $50 \mathrm{mmHg}$ in non-diseased arterial walls, the adventitia and close to the lumen of atherosclerotic plaques $(8,9)$.

The presence of plaque hypoxia is nowadays widely accepted and has been shown across species. However, whether pimonidazole staining and plaque hypoxia represent pathological or physiological hypoxia has not yet been clarified. For tumors the critical partial oxygen pressure is proposed to be $8-10 \mathrm{mmHg}$ though (10), and below this threshold, ATP depletion, intracellular acidosis and apoptosis can be observed. Similarly advanced rabbit plaques ( $<500 \mu \mathrm{m}$ thick) showed ATP depletion, low glucose and glycogen concentrations and lactate accumulation. Additionally these plaques were pimonidazole positive (11). In line with that, Naghavi et al. could show a significantly lower $\mathrm{pH}$ in lipid-rich areas as compared to calcified areas in human carotid endarterectomy specimens and a generally high spatial heterogeneity in $\mathrm{pH}$ within human and rabbit plaques (12). All in all, at least rabbit and human atherosclerotic plaques show signs of pathological hypoxia, with energy depletion and acidosis as main features. This suggests a causative role of hypoxia in plaque progression and thus oxygen therapy would be expected to reduce plaque burden.

First proof for the applicability of oxygen therapy in atherosclerosis comes from as early as 1954. Altschul et al. showed that exposure of hypercholesterolemic rabbits to intermittent $60-65 \%$ oxygen reduced atherosclerosis development, by significantly reducing plasma cholesterol levels (13). Vesselinovitch et al. later studied plaque regression in rabbits. When switching high cholesterol diet fed rabbits back to normal diet, they could show profound plaque regression. Plaque regression was even more 
pronounced, when adding oxygen therapy (100\% oxygen) on of top of the diet-switch and these plaques presented with a high proportion of collagen, a thick fibro-muscular cap and mild to moderate lipid deposition in the aorta (14).

More recently, subjecting mice to normoxia after 6 weeks of intermittent hypoxia could reverse cardiovascular remodeling seen upon intermittent hypoxia (15). Similarly, reversal of chronic intermittent hypoxia $(\mathrm{CIH})$ in apnea patients using continuous positive airway pressure (cPAP) could improve cardiac symptoms and hemodynamic parameters $(16,17)$. cPAP can increase daytime partial oxygen pressure by substantially reducing the number and severity of respiratory events in patients suffering from $\mathrm{CIH}$ (18). Additionally, hyperbaric oxygen therapy, using 100\% oxygen, has been shown to reduce atherosclerotic formation in rabbits and mice (19-21). This is in line with our results, where we showed reduced necrotic core expansion in mice receiving carbogen gas (chapter 3). Hyperbaric oxygen exerted its anti-atherosclerotic effect by reducing lipid oxidation (19), and ox-LDL specific auto-antibody titers (20) and by dampening $\mathrm{T}$ and $\mathrm{B}$ cell mediated response to oxLDL and inflammatory stimuli (21), while plasma cholesterol levels remained unchanged (19-21). While we did not measure autoantibodies against oxLDL or $\mathrm{T}$ and $\mathrm{B}$ cell responses to oxLDL, we did not observe any changes in systemic cytokine levels. Also, we did not observe differences in intra-plaque or serum reactive oxygen species production in chapter 3, pointing towards unaltered lipid oxidation. However, we cannot exclude that carbogen gas affected auto-antibody production or $\mathrm{T}$ and $\mathrm{B}$ cell responses.

In turn, we could show reduction of plaque hypoxia by our treatment, which was not investigated in any previous studies. Improving oxygen delivery enhanced efferocytosis capacity by macrophages in vivo. When studying this phenomenon in vitro, we could show reduced expression of the efferocytosis receptor mer tyrosine kinase (MerTK) in hypoxic conditions, likely explaining the in vivo phenotype in the plaque (chapter 3). Indeed, MerTK dysfunction due to mutations resulted in elevated plaque necrosis and apoptotic cell accumulation in mice (22). However, reversal of MerTK activity and its effect on atherosclerosis and thus translation to the in vivo situation remains to be established.

Overall, systemic oxygen therapy seems to alleviate atherosclerotic and cardiovascular disease burden. Depending on the route of administration and percentage of oxygen administered, oxygen therapy reduces hypercholesterolemia, inflammation and even hypertension $(13,16,17,21)$. Unfortunately, the chronic nature of atherosclerosis and its prevalence make oxygen therapy not feasible as general therapy for atherosclerosis and 
alternative routes have to be explored. Alternatively, targeting downstream factors in the hypoxic signaling cascade, e.g. enhancing MerTK availability and/or activity in the plaque might reduce plaque progression.

\section{CELLULAR OXYGEN CONSUMPtION AND INFLAMMation IN Atherogclerotic PLAQUES}

Plaque hypoxia can result from decreased oxygen diffusion into the plaque or enhanced cellular oxygen consumption. With an oxygen diffusion distance of about 100-250 $\mu \mathrm{m}$ (23), oxygen diffusion distance is unlikely to determine plaque hypoxia in murine plaques (max. $200 \mu \mathrm{m}$ thick). Indeed, murine plaques showed pimonidazole-positivity from 50 $\mu \mathrm{m}$ thick fatty streak formation onwards, suggesting enhanced oxygen consumption rather than oxygen diffusion to underlie plaque hypoxia (chapter 3). Oxygen is for example required for LDL oxidation in vitro (24), though this effect might be marginal in the plaque, as for the plaque mainly radicals and enzymatic modifications have been described in LDL oxidation, including reactive nitrogen species, as well as myeloperoxidase and lipoxygenases (25). Alternatively, M2 macrophages with maintained oxidative phosphorylation (reviewed in (26)), could be responsible for consuming oxygen in plaques. Activated more pro-inflammatory peritoneal macrophages actually present with reduced oxygen consumption as compared to resting cells (27). Additionally, M1 macrophages show anaerobic metabolism, suggesting reduced cellular oxygen consumption (28).

In atherosclerotic plaques, both pro-inflammatory M1 and anti-inflammatory M2 macrophages are found, with M1 macrophages being localized in rupture-prone areas (29). Hypoxia mainly co-localized with macrophages in atherosclerotic plaques in humans (30) and mice (chapter 3) and inflammation and hypoxia are in general tightly interlinked: inflammatory diseases are frequently characterized by tissue hypoxia (e.g. in inflammatory bowel disease and lung inflammation $(3,31,32))$ and vice versa hypoxic conditions, such as ischemia/reperfusion injury, show enhanced inflammatory influx (33). Also, short term exposure to low oxygen concentration led to enhanced vascular leakage, inflammatory cell accumulation in multiple organs, as studied upon Evan's blue leakage into abdominal organs, and elevated serum cytokine levels in experimental mouse models (34-38). In particular for pro-inflammatory macrophages in vitro the relationship between glycolysis, inflammation and hypoxia is evident. However, whether this hypoxia is secondary to macrophage inflammation or proceeds inflammation is unknown. Tawakol et al. could recently show that hypoxia enhances macrophage glycolysis and favored a pro-inflammatory macrophage M1 phenotype. In turn, inhibiting glycolysis 
using the 6-phosphofructo-2-kinase-fructose-2,6-biphosphatase 3 (PFKFB3) inhibitor 3PO, reversed pro-inflammatory M1 activation both in normoxia and hypoxia (28). Whether glycolysis inhibition also affects macrophage oxygen consumption, however, was not assessed. In turn, they could show reduced cellular oxygen consumption and enhanced glycolysis in macrophages exposed to oxLDL in vitro (28). It remains to be studied whether oxLDL exposure also enhances non-oxygen consuming glycolysis in vivo in macrophages. This would then suggest that the hypoxia observed in fatty streak does not result from foam cell formation. Indeed also in our hands, oxLDL-mediated foam cell formation did not promote pimonidazole-positivity in vitro (chapter 3). A more detailed time line, tracing pimonidazole positivity in the initial phases of murine atherosclerosis development might give insight into this chicken-egg situation of inflammation and hypoxia in atherosclerosis.

Independent of the origin of plaque hypoxia, hypoxia can have effects on the microenvironment of the plaque, such as inducing angiogenesis, but also amplifying inflammation in the plaque. Thus, reducing cellular oxygen consumption was hypothesized to reduce plaque progression.

However, our attempts to reduce cellular oxygen consumption (chapter 4, 5, 6) within the plaque, by genetic or pharmacological intervention, were unable to proof or disproof a causative effect of hypoxia in atherosclerosis development, angiogenesis and inflammation, due to multiple reasons: In chapter 4, prolonged dietary nitrate supplementation did not affect plaque hypoxia or size. Short term dietary nitrate supplementation has been shown to improve mitochondrial function, thereby reducing oxygen requirement during exercise (39-41). However, a compensatory downregulation of plasma nitrate, nitrite and nitric oxide levels after 14 weeks of dietary nitrate supplementation obscured any effects on plaque hypoxia. How elimination or processing of NOx intermediates occurs remains to be studied. Plasma nitrate and nitrite levels were elevated after 2 weeks of supplementation (chapter 4) and short term dietary nitrate administration has been shown to lower blood pressure as well as exert antiinflammatory effects, as studied in inflammatory bowel disease models $(42,43)$. While we did not study effects of short term dietary nitrate supplementation on blood pressure, oxygen consumption ability or inflammation in our model, it seems that none of the effects described were sufficient to affect atherosclerotic plaque hypoxia or progression. Thus, from this study no firm conclusions on the effect of plaque oxygen consumption on plaque progression could be drawn. 
In chapter 6, PHD3 deficiency did also not affect plaque hypoxia in mice. PHD3 deficiency is known to result in stabilization of the transcription factor HIF, with subsequent transcription of genes involved in erythropoiesis, angiogenesis, energy metabolism as well as oxygen delivery and oxygen consumption (44). While plaque hypoxia was unchanged by PHD3 deficiency, we did not directly measure oxygen consumption in any PHD3 lacking plaque-relevant cells (e.g. macrophages, smooth muscle cells). Thus, we cannot conclude whether oxygen consumption in PHD3 lacking cells was actually affected. Overall, this model does not allow conclusions about a role of hypoxia in atherosclerosis.

In the PHD1 deficiency model, with known reduction in cellular oxygen consumption (45), a reduction in plaque hypoxia was observed (chapter 5). However, when matching mice for cholesterol levels, there was no significant additional PHD1 deficiency mediated effect seen on plaque size, necrotic core size (Figure 1). Thus, the cholesterol-lowering effect likely trumped all direct hypoxia-mediated effects on plaque progression.
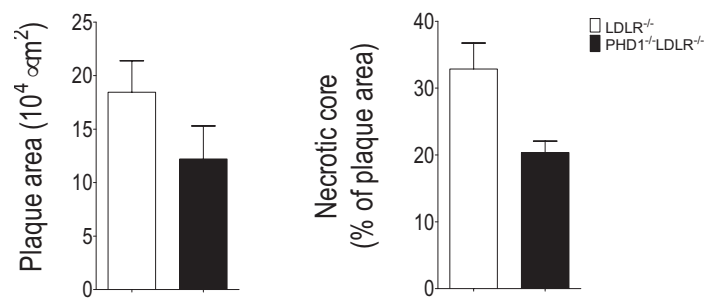

\section{Figure 1:}

Atherosclerotic plaque size and necrotic core content of PHD1/ LDLr/ mice and controls in the aortic roots of cholesterol-matched mice.

To conclude, the models used in this dissertation did neither proof nor disproof a causal contribution of cellular oxygen consumption on plaque progression. While reducing cellular oxygen consumption in the PHD1 deficiency model could reduce plaque hypoxia, the cholesterol-lowering effects overruled plaque hypoxia effects. This might suggest a more modulatory role of plaque hypoxia. Potentially in line with a modulatory role of plaque hypoxia, plaque oxygenation with carbogen gas treatment in chapter 3 affected plaque phenotype by reducing necrotic core formation, but not plaque size. 
Induction of HIF signaling would be expected to promote angiogenesis in our PHD deficiency models (44). However, none of the models presented here, show intraplaque angiogenesis (chapters 3-6). This is a general drawback of most current murine atherosclerosis models, which complicates translation to the human situation, where neo-vessel formation has been associated with symptomatic carotid disease (thus plaque prone to rupture) (46-49). On the one hand, we might thus underestimate the role of hypoxia in atherosclerotic plaques in LDLr deficiency mouse models, which might be mediated by enhanced angiogenesis in humans. On the other hand, we might not observe any changes in plaque hypoxia in our PHD models due to the lack of dysfunctional microvessel formation in murine plaques. Indeed, PHD3 deficiency would be expected to induce angiogenesis, as shown in a murine hindlimb ischemia model (50), and thus formation of potentially oxygen carrying vessels in the plaque. Thus PHD3 inhibition in the human situation might actually induce or facilitate plaque angiogenesis, which is not observed in our murine models. However, plaque hypoxia is also present in human plaques, independent of angiogenesis (48). Thus, even in the case of microvessel formation, angiogenesis is either not sufficient or dysfunctional in restoring plaque normoxia.

Interestingly, a link between hypoxia and angiogenesis or vice versa has never been shown for atherosclerosis. In turn, also plaque angiogenesis itself has never actually been causally linked to atherosclerosis either and is only "guilty by association" in plaque progression and vulnerability (51). Best evidence comes from experimental studies in aged ApoE deficient mice on high cholesterol diet, using anti-angiogenic therapy $(52,53)$, which could reduce atherosclerosis development. However, numbers and incidences of intra-plaque vessels angiogenesis were very low and non-specific effects of the therapy cannot be excluded. Also, studies on pro-angiogenic therapy successfully increasing angiogenesis and potentially atherosclerosis remain to be performed. A recent murine model with heterozygous mutations in the fibrillin-1 gene actually developed intra-plaque and adventitial angiogenesis, hemorrhage and plaque rupture. Also, these mice showed plaque hypoxia, which mainly localized to sites of angiogenesis (54). While the authors suggest hypoxia to precede angiogenesis in that case, proof of causality is still outstanding. 


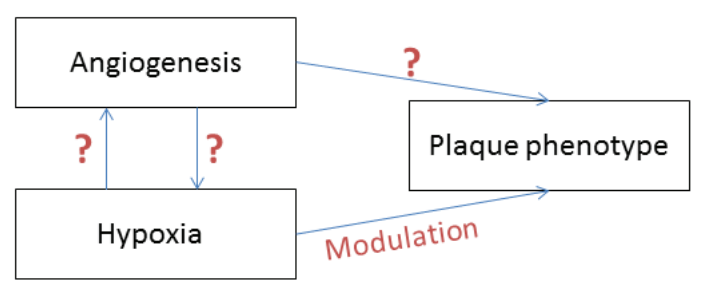

Figure 2:

Triangle of angiogenesis, hypoxia and plaque phenotype. Causal relations have not been shown between hypoxia resulting in plaque angiogenesis or vice versa (e.g. via enhanced inflammation). Also plaque angiogenesis has never been causally shown to affect plaque phenotype or even promote plaque rupture.

In this triangle of unknowns (Figure 2), we can only conclude about angiogenesisindependent effects of plaque hypoxia from our models, pointing towards a modulatory role of plaque hypoxia. Recent advances in murine but also pig models with angiogenesis offer promising tools to tackle causal relationships between hypoxia, angiogenesis and plaque phenotype. Indeed, pig plaques very much resemble human atherosclerosis and the PCSK9 Yucatan mini pig has recently been shown to present with intra-plaque, as well as adventitial vessel growth and hypoxia (55). Local angiogenesis inhibition of intra-plaque and adventitial plaque angiogenesis e.g. using VEGFR and PDGFR inhibitors in those models will give more insight into a causal relationship between angiogenesis, plaque hypoxia and plaque progression. 


\section{PHDS IN CARDIOVASCULAR DISEASE}

In this dissertation, we present evidence for differential roles of PHD1 and PHD3 inhibition in atherosclerosis (chapters 5 and 6). Additionally, our group has studied myeloid PHD2 deficiency in atherosclerosis (Theelen, Marsch et al. in preparation). In line with the PHD isoform diversity (discussed in chapter 1), the three isoforms have very different effects on atherosclerotic phenotypes (table 1):

\begin{tabular}{|c|c|c|c|c|c|c|c|}
\hline Parameter & $\begin{array}{l}\text { PHD1 WB } \\
\text { (chapter 5) }\end{array}$ & $\begin{array}{l}\text { PHD1 BMT } \\
\text { (chapter 5) }\end{array}$ & $\begin{array}{l}\text { PHD2 } \\
\text { inhibitor* }\end{array}$ & $\begin{array}{l}\text { PHD2 } \\
\text { hypomorph* }\end{array}$ & $\begin{array}{l}\text { PHD2 mf } \\
\text { (submitted) }\end{array}$ & $\begin{array}{l}\text { PHD3 WB } \\
\text { (chapter 6) }\end{array}$ & $\begin{array}{l}\text { PHD3 BMT } \\
\text { (chapter 6) }\end{array}$ \\
\hline plaque size & $\downarrow$ & $=$ & $\downarrow$ & $\downarrow$ & $\uparrow$ & $=$ & $\uparrow$ \\
\hline plasma cholesterol & $\downarrow$ & $=$ & $\downarrow$ & $\downarrow=* *$ & $\downarrow$ & $\uparrow$ & $=$ \\
\hline apoptosis & ND & ND & ND & ND & ND & ND & $\uparrow$ \\
\hline erythropoiesis & $=$ & $=$ & ND & ND & $=$ & 个 НСТ & $=$ \\
\hline
\end{tabular}

Tabe 1: PHDS in murine $\mathrm{LDLr}^{-/}$atherosclerosis models in vivo.

* from (56) ** reduced on chow (57), not on high fat diet (56). BMT - bone marrow transplantation model; HCT bematocrit; $m f$ - myeloid-specific deficiency; ND - not determined; WB - whole body deficiency model.

PHD1 deficiency reduced atherosclerosis development by reducing plasma cholesterol levels, an effect that has also recently been described for whole body PHD2 inhibition (56). Interestingly, myeloid PHD2 deficiency also reduced plasma cholesterol levels slightly after 12 weeks of high cholesterol diet, but increased atherosclerotic plaque size (Theelen, Marsch et al. in preparation). Whole body PHD3 deficiency showed no effect on atherosclerosis but enhanced plasma cholesterol, while the hematopoietic PHD3 deficiency increased plaque size with only a trend towards increased plasma cholesterol levels. On the contrary, hematopoietic PHD1 deficiency did not affect atherosclerosis development or cholesterol at all. Thus, there seems to be a cell type-specific and isoenzyme-specific effect on plasma cholesterol levels and atherosclerosis development (figure 3). As the PHD isoforms have the same substrates, expression pattern could underlie the differential effects seen. However, in general all three PHD isoforms are ubiquitously expressed, with PHD2 expression exceeding PHD1 and PHD3 in most organs. A particular enhancement in expression is seen for PHD1 in testes and PHD3 in the heart (www.genecards.org) and latter could potentially contribute to the phenotype seen in PHD3 deficient mice. 
In chapter $1, \mathrm{I}$ already described the diverse functions of the three PHD isoforms in ischemia/reperfusion (I/R). In that setting, PHD2 deficiency seems to mainly induce angiogenesis, thereby reducing $\mathrm{I} / \mathrm{R}$ injury. In our model of myeloid PHD2 deficiency, however, we did not observe any effects on angiogenesis, quantified as CD31 positive microvessels in the adventitia (table 1). The authors of PHD2 inhibition and hypomorphism paper did not study angiogenesis $(56,57)$. This does not exclude a pro-angiogenic activity of PHD2 inhibition in humans though, as in general there are no good murine models of plaque angiogenesis available (reviewed elsewhere (58-66)). In line with a PHD2-specific role in angiogenesis, plaque angiogenesis was not affected in whole body PHD1 deficient mice or hematopoietic PHD3 deficient mice (table 1, chapter 5 and 6), though again the limitations of the model have to be considered. Against expectations, myeloid PHD2 deficiency showed enhanced macrophageassociated collagen production, likely via HIF-1 $\alpha$ stabilization (Theelen, Marsch et al. in preparation). In line with these results, hypoxia has recently been shown to increase collagen synthesis in cultured fibroblasts (67) and enhance collage cross-linking (68). These phenotypes were not seen in PHD1 or PHD3 deficiency models (both BMT and whole body, chapter 5 and 6), again illustrating major cell type and isoform differences.

In contrast, hematopoietic PHD3 deficiency resulted in enhanced plaque size with enhanced necrotic core expansion and apoptosis in the plaque (chapter 6). This is in line with the literature, linking PHD3 to intracellular apoptosis signaling, with PHD3 seemingly differentially regulating apoptosis in hypoxia and normoxia. On the one hand, PHD3 silencing in vitro protected normoxic rat cardiomyocytes from doxorubicininduced apoptosis. Consequently, PHD3 overexpression induced apoptosis by inhibiting the formation of the anti-apoptotic protein complex Bax/Bcl-2 (69). In line with that, normoxic sympathetic neurons from PHD3 deficient mice showed HIF- $2 \alpha-$ dependent enhancement of cell survival. Also in vivo, PHD3 deficient mice presented with increased superior cervical ganglia numbers, though they were not functional (70). PHD3 deficient macrophages also show improved survival in response to stress triggers (serum-starvation, staurosporine and SNAP) in normoxic conditions (71). On the other hand, in hypoxic neutrophils, PHD3 deficiency resulted in enhanced selective apoptosis of neutrophils in vivo and in vitro, this time by reducing $\mathrm{Bcl}$ family member Bcl-xl expression and increasing pro-apoptotic Siva1 expression (72). Hypoxia in atherosclerotic plaques may thus promote the pro-apoptotic phenotype seen in hematopoietic PHD3 deficient mice in chapter 6. Neither whole body PHD3 nor PHD1 deficiency nor myeloid PHD2 deficiency seemed to affect apoptosis in a similar way (chapter 5). 
PHD1 deficiency has been linked to a switch in cellular metabolism towards glycolysis (45). The subsequent reduced cellular oxygen consumption then protected against ischemic necrosis in a femoral artery ligation model (45). Also in atherosclerosis, PHD1 deficiency reduced plaque hypoxia, presumably by reducing oxygen consumption. However, hematopoietic PHD1 deficiency did not affect plaque hypoxia (chapter 5).

This is intriguing and suggests a decisive role of macrophages in plaque hypoxia. This could mean that PHD1 deficient macrophages do not show reduced cellular oxygen consumption in the plaque in first place and that other stromal cells lacking PHD1 affected macrophage hypoxia in the whole body deficiency, which was not seen in the hematopoietic PHD1 deficiency only. Alternatively, reduced plaque size, earlier plaque stage, inflammatory cell count or cholesterol-lowering seen in whole body PHD1 deficient mice, but not in hematopoietic PHD1 deficiency, could determine the relative decrease in plaque hypoxia upon whole body PHD1 deficiency. Additionally, pimonidazole only detects oxygen tensions below $1 \%$ oxygen $(<10 \mathrm{mmHg})$ in the tissue and small oxygenation changes exerted by hematopoietic PHD1 deficiency might not be visible in the staining. While plaque hypoxia was not determined in whole body PHD2 hypomorphic mice (56,57), myeloid PHD2, hematopoietic PHD3 and whole body PHD3 deficiency also did not affect plaque hypoxia. Thus stromal PHD1 seems to regulate atherosclerotic plaque hypoxia via a yet unknown mechanism. Studying cellular oxygen consumption under hypoxic conditions and upon atherosclerotic triggers (e.g. oxLDL, interleukins and TNF- $\alpha$ ) in PHD deficient macrophages might give insight into whether these macrophages actually show reduced cellular oxygen consumption in the plaque. Co-cultures with smooth muscle cells or total plaque lysates could reveal an additional effect of the plaque microenvironment on cellular oxygen consumption. To investigate whether plaque size, stage and/or cholesterol levels affect plaque hypoxia, non-invasive imaging of hypoxia progression in plaques over time could be considered, with paralleled tracing of plasma/serum cholesterol levels. 


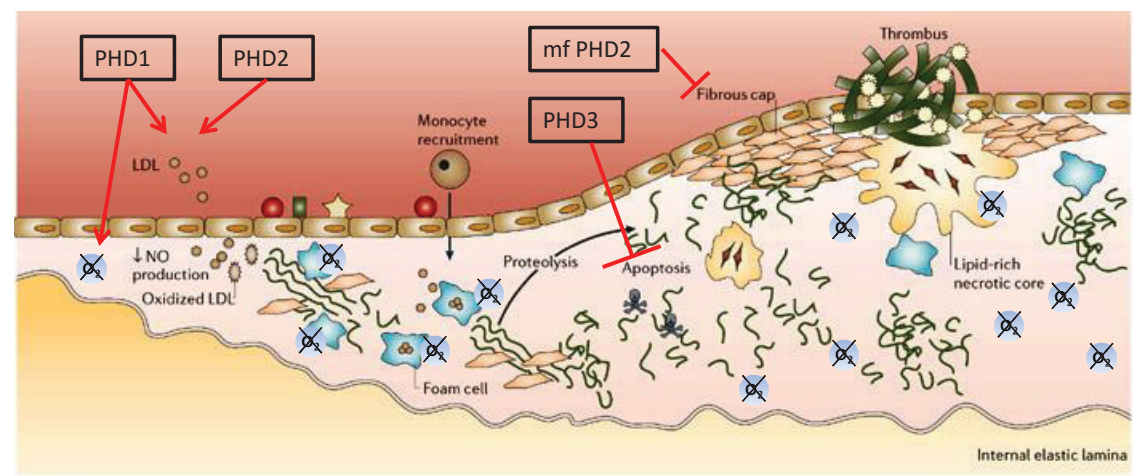

\begin{tabular}{lll}
\hline Apoptotic cell & Collagen fibril \\
Platelet & Smooth muscle cell
\end{tabular} Fibrin 2 Macrophage

Figure 3: Adjusted from (73), indicateing differential functions of PHDs in atherosclerosis.

To conclude, PHD isoforms have distinct effects on plaque growth and affect different atherosclerotic processes. PHD3 deficiency opposes the effects of PHD1 and PHD2 on plaque development and more obviously on plasma cholesterol levels. This might be explained by the fact, that PHD3 is actually upregulated in hypoxia. Also PHD3 has a lower oxygen $\mathrm{Km}$ value (concentration of oxygen required to achieve $50 \%$ of the maximum enzymatic activity) and might thus still be active at intermediate oxygen tensions (74,75), thereby regulating HIF responses in hypoxia. PHD3 inhibition therefore prevents the hypoxic feedback response (74) and might result in uncontrolled HIF responses. Investigating cellular oxygen consumption as well as HIF stabilization in PHD3 deficiency macrophage and/or hepatocytes might give more insight into mechanisms underlying enhanced atherosclerosis and cholesterol levels.

Differential affinity of PHD isoforms for HIF- $1 \alpha$ and HIF- $2 \alpha$ hydroxylation (76) could potentially explain their diverse functions in atherosclerosis. HIF- $1 \alpha$ and HIF-2 $\alpha$ are expressed in human plaques and correlate with plaque hypoxia and macrophages (30). HIF- $2 \alpha$ deficiency has so far not been studied in atherosclerosis and plaque hypoxia was only assessed in one study, where macrophage-specific HIF-1 $\alpha$ deficiency did not affect hypoxia (unpublished data). HIF-1 $\alpha$ effects on atherosclerosis are summarized in table 2 . Overall, HIF-1 $\alpha$ deficiency also cell type-specifically and non-consistently affects plaque size, complicating conclusions on whether PHD effects on atherosclerosis are HIF-mediated. 


\begin{tabular}{|c|c|c|c|c|c|c|}
\hline HIF-1 $\alpha$ modulation & Cell type & Model & $\begin{array}{l}\text { Plaque } \\
\text { size }\end{array}$ & $\begin{array}{l}\text { Plasma } \\
\text { cholesterol }\end{array}$ & Identified process & Ref \\
\hline Ко & $\begin{array}{l}\text { VE-Cad-Cre- } \\
E R^{T 2} E C\end{array}$ & $\begin{array}{l}\text { ApoE }{ }^{-/} \text {, tamoxifen-induced } \\
\text { KO prior to start of diet }\end{array}$ & $\downarrow$ & $=$ & $\begin{array}{l}\downarrow \text { monocyte adhesion } \\
\text { macrophage accumulation }\end{array}$ & (77) \\
\hline HIF- $\alpha$ KO & $\begin{array}{l}\text { Local } \\
\text { adenovirus }\end{array}$ & $\begin{array}{l}\text { ApoE }{ }^{-1-} \text { wire-induced vascular } \\
\text { injury }\end{array}$ & $\downarrow$ & ND & $\downarrow$ VEGF-A expression & (78) \\
\hline HIF- $\alpha$ KI & $\begin{array}{l}\text { Local } \\
\text { adenovirus }\end{array}$ & $\begin{array}{l}\text { ApoE }{ }^{-/-} \text {wire-induced vascular } \\
\text { injury }\end{array}$ & $\uparrow$ & ND & ND & (78) \\
\hline KI & $\begin{array}{l}\text { Mainly } \mathrm{CD} 4^{+} \mathrm{T} \\
\text { cells }\end{array}$ & $\begin{array}{l}\text { ApoE }{ }^{-\%} \text {, systemic plasmid } \\
\text { infusion, mainly accumulates } \\
\text { in } \mathrm{CD}^{+} \mathrm{T} \text { cells }\end{array}$ & $\downarrow$ & $=$ & $\begin{array}{l}\downarrow \text { IFN- } \gamma \text { in } \mathrm{CD}^{+} \mathrm{T} \text { cells and } \\
\text { aortas }\end{array}$ & (79) \\
\hline Ко & $\mathrm{CD} 11 \mathrm{c}^{+} \mathrm{cDC} / \mathrm{mf}$ & $\operatorname{LDLr}^{-/}$ & $\uparrow$ & $=$ & $\begin{array}{l}\text { Increased T cell } \\
\text { recruitment, likely } \\
\text { attributable to } \mathrm{cDCs}\end{array}$ & (80) \\
\hline KO & LysMCre Mf & $\operatorname{LDLr}^{-/-}$ & $=$ & $=$ & $\begin{array}{l}\text { No effect on plaque } \\
\text { hypoxia }\end{array}$ & (80) \\
\hline KO & $\begin{array}{l}\text { Local HIF-1 } \alpha \\
\text { SiRNA }\end{array}$ & $\begin{array}{l}\text { ApoE }{ }^{-/-} \text {wire-induced vascular } \\
\text { injury }\end{array}$ & $\downarrow$ & ND & $\begin{array}{l}\text { Reduced smooth muscle } \\
\text { cell content }\end{array}$ & (81) \\
\hline HIF- $2 \alpha$ stabilization & Liver & C57BL/6, vHL deletion & ? & $\uparrow$ & $\begin{array}{l}\text { Decreased bile acid } \\
\text { synthesis and signaling }\end{array}$ & (82) \\
\hline HIF-1 $\alpha$ stabilization & Liver & C57BL/6, vHL deletion & ? & $=$ & HIF- $1 \alpha$ stabilized & (82) \\
\hline HIF- $\alpha$ stabilization & Intestine & C57BL/6, vHL deletion & $?$ & $=$ & HIF- $1 \alpha \&$ HIF- $2 \alpha$ stabilized & (82) \\
\hline HIF- $\alpha$ stabilization & Mf & C57BL/6, vHL deletion & ? & $=$ & HIF- $1 \alpha$ \& HIF- $2 \alpha$ stabilized & (82) \\
\hline
\end{tabular}

Table 2: HIFs in atherosclerosis and plasma cholesterol regulation in vivo.

$K O$ - deficiency; KI - knockin; $c D C$ - conventional dendritic cell; $m f$ - macrophage; $N D$ - not determined; ApoE - apolipoprotein E; vHL - von Hippel Lindau; VEGF-A - vascular endothelial cell growth factor A; IFN- $\gamma$ interferon $\gamma$.

In conclusion, both PHDs and HIF-1 $\alpha$ have cell type-specific effects on plaque development and progression, which need to be considered individually in order to draw mechanistic conclusions. All PHD deficiencies show HIF- $\alpha$ stabilization $(76,83)$ and PHD interference may skew the HIF-stabilizing system, potentially explaining phenotypic differences between HIF deficiency and PHD deficiency models. In fact, PHD isoform inhibition reflects a much more subtle intervention as compared to HIF interference, as the remaining PHD isoforms can still fullfil their task in HIF hydroxylation. Next to that, PHDs have additional binding partners and potentially even hydroxylation substrates (discussed in chapter 1), which could exert effects on atherogenesis. Thus, we are only beginning to understand the oxygen sensors PHDs in atherosclerosis and other disease conditions. Further cell type-, tissue- and PHD isoform-specific studies will reveal further applicability of these proteins as therapeutic targets. 


\section{HYPOXIA AND CHOLESTEROL METABOLISM}

Interestingly, we could show a novel contribution of oxygen sensor PHDs to cholesterol metabolism regulation in chapters 5 and 6 . These data suggest a link between hypoxia and cholesterol metabolism. Indeed, on the one hand, oxygen breathing in rabbits and humans seemed to decrease plasma cholesterol levels, as presented already in the $1950 \mathrm{~s}$ by Altschul (13), and exposure of mice to continuous (82) or intermittent hypoxia has actually enhanced serum cholesterol levels (84). On the other hand, reversing intermittent hypoxia using continuous positive airway pressure in sleep apnea patients reduced serum cholesterol levels in one randomized clinical trial (85), but not in three others $(17,86,87)$. These diverse outcomes of hypoxia on cholesterol metabolism might not be surprising, considering the complexity of cholesterol metabolism involving crosstalk of multiple organs (including liver, intestines but also peripheral tissue) as well as multiple processes (cholesterol synthesis, reverse cholesterol transport, lipoprotein metabolism, cholesterol absorption etc.),

Hematopoietic deficiencies did not affect plasma cholesterol levels, except a minor decrease in plasma cholesterol levels for the myeloid PHD2 deficiency model after 12 weeks high cholesterol diet (Theelen, Marsch et al. in preparation). Despite this cholesterol-lowering, mice actually presented with larger plaques. Hematopoietic cells can affect cholesterol metabolism e.g. in the liver, where Kupffer cells can take up cholesterol in high cholesterol fed rabbits (88). Indeed, Kupffer cell depletion could prevent steatosis (89), showing a role in cholesterol metabolism. To what extent these hepatic macrophages can contribute to cholesterol clearance from plasma however is unknown. Next to using cholesterol for cellular functions, peripheral cells including macrophages contribute to cholesterol metabolism is reverse cholesterol transport (RCT). Macrophages can affect RCT via increased cholesterol efflux to HDL mainly. This process is mediated by ABCA1 and ABCG1 and potentially SR-B1, though the latter is not yet confirmed in vivo (90). Mechanistically, Parathath and colleagues could show that hypoxia and subsequent HIF-1 $\alpha$ stabilization actually decreased ABCA-1 expression levels with subsequent reduction in cholesterol efflux to ApoA1 (a form of pre- $\beta$-HDL) in vitro. In line, HIF- $1 \alpha$ over-expression reduced cholesterol efflux from macrophages, which was even further decreased upon hypoxia in vitro (91). This would suggest reduced cholesterol efflux in PHD2 deficient macrophages in our model (Theelen, Marsch et al. in preparation), but cholesterol efflux capacity of PHD2 deficient macrophages remains to be confirmed. On top of that, hypoxia and HIF stabilization have been shown to enhance cholesterol accumulation in multiple cell types and organs. Hypoxic macrophages showed enhanced cholesterol uptake via Lox-1 upon hypoxia (92), and RNA interference with HIF-1 $\alpha$ could inhibit foam cell formation in vitro (93). 
In our model of macrophage-specific PHD2 deficiency, we did not investigate foam cell formation (Theelen, Marsch et al. in preparation) and overall, the mechanism underlying macrophage PHD2-mediated cholesterol lowering remains to be established. Against a possible role of $\mathrm{HIF}-1 \alpha$, are the data from Chaudhari et al., who showed no effect of lysMCre HIF-1 $\alpha$ deficiency bone marrow transplantations on plasma cholesterol levels after 6 weeks of $1.25 \%$ cholesterol diet (80). Likewise, macrophage-specific von Hippel Lindau deletion, resulting in both HIF- $1 \alpha$ and HIF- $2 \alpha$ stabilization, did not affect plasma cholesterol levels either (82). Thus, the cholesterol-lowering seen in myeloid PHD2 deficient mice might not be mediated by HIFs (Theelen, Marsch et al. in preparation). Neither hematopoietic PHD1 nor PHD3 deficiency show an effect on plasma cholesterol levels. PHD3 is actually higher expressed in peritoneal macrophages (www.immgen.org), which could however not result in a cholesterol phenotype. Thus PHD2 function rather than expression abundance seems critical for macrophagemediated effects on cholesterol metabolism.

Intriguingly, while PHD1 and PHD2 whole body deficiencies reduce plasma cholesterol levels (chapter 5, (56,57), PHD3 ablation had an opposite, albeit modest effect on plasma cholesterol (chapters 6). The relatively small effect size in PHD3 deficiency might be due to the already oversaturated plasma cholesterol levels of mice on high cholesterol diet. Alternatively, PHD3 activity or expression might not be sufficient to affect lipid profiles to the same extend as PHD1 and PHD2.

Interestingly, the cholesterol synthesis pathway is generally considered a highly oxygenconsuming pathway $(94,95)$ and accordingly, hypoxic kidney fibroblast-like cell lines and ovarian epithelial cell-like cells (1\% oxygen) showed enhanced lanosterol accumulation and subsequent degradation of HMG-CoA reductase. HMG-CoA reductase presents the rate-limiting step in cholesterol synthesis and upon degradation, cholesterol synthesis is presumably reduced (96). Tracing glucose and glutamine derived carbons showed reduced incorporation into cholesterol from glucose and glutamine in a metastatic histiocytoma cell line upon hypoxia, suggesting reduced cholesterol synthesis at least from these precursors (97). Alternatively, hepatic HMG-CoA levels in rats exposed to whole body hypoxia were not altered, suggesting unchanged hepatic cholesterol synthesis (98). In contrast, Parathath et al. could show increased HMG-CoA reductase expression in hypoxia macrophages in vitro. This upregulation was also HIF dependent and resulted in increased cholesterol synthesis in hypoxic, HIF-1 $\alpha$ positive macrophages (91). Thus, macrophages seem to increase cholesterol synthesis upon HIF-1 $\alpha$ stabilization, while in other cell-types cholesterol synthesis is unchanged or even decreased. Indeed, Rahtu-Korpela et al. suggest hampered hepatic cholesterol synthesis as the underlying 
cause of plasma lowering in PHD2 hypomorphic mice (56,57). However, these cholesterol synthesis measurements should be considered with caution. Simultaneous alterations in other cholesterol handling pathways, such as reduced cholesterol efflux, can affect the intracellular cholesterol pool, thereby affecting the intracellular tracer concentration. Correction for the appropriate cholesterol pool (if measuring tracer accumulation in plasma, thus plasma cholesterol pool) was indeed not considered in the study by RahtuKorpela et al. and the data should be validated. In our studies, we corrected for the total plasma contained cholesterol levels (pool) when analyzing ${ }^{13} \mathrm{C}$-incorporation into cholesterol in plasma. Based on this analysis, whole body cholesterol synthesis was not altered by PHD1 deficiency (chapter 5) and whether cholesterol synthesis is altered in PHD2 or PHD3 deficiency remains to be established.

PHD3 ablation has been shown to stabilize HIF-2 $\alpha$ in livers (99) and cholesterol elevation might indeed be HIF- $2 \alpha$-mediated. Hypoxic HepG2 showed enhanced lipid and cholesterol accumulation upon HIF-2 $\alpha$ mediated ABCA1 downregulation in vitro (100). In line with that, liver weights were much increased in whole body PHD3 deficient mice (chapter 6). In vivo, Ramakrishnan et al. found that HIF $\alpha$ stabilization obtained by postnatal hepatic von Hippel Lindau (vHL)-deletion enhanced hepatic and plasma cholesterol levels. Effects were mainly dependent on HIF-2 $\alpha$-mediated limitation of bile acid metabolism and biliary cholesterol clearance (82). Also in our hands, combined hepatic and intestinal HIF- $2 \alpha$ knockdown could increase plasma cholesterol levels in LDLr deficient mice but not in PHD1 deficient animals (chapter 5). Thus, PHD3 deficiency-mediated HIF-2 $\alpha$ stabilization is likely to promote the hypercholesterolemia seen, while cholesterol-lowering in PHD1 consequently HIF-2 $\alpha$ independent. Interfering with hepatic HIF-2 $\alpha$ expression in PHD3 deficient mice (e.g. via antisense oligonucleotides) would reveal whether PHD3 deficiency mediated cholesterol-raising is HIF-2 $\alpha$ dependent.

Overall, whole body PHD1 and PHD2 interference protected from diet-induced hypercholesterolemia (chapter 5, (56)). Also in the human situation general inhibition of all PHDs by Roxadustat and GSK1278863 mirrored the cholesterol-lowering effect seen in PHD1 and PHD2 deficient mice $(101,102)$. This data again highlights the delicate balance of PHD induced HIF hydroxylation. While PHD3 deletion, as well as hepatic vHL deficiency, with subsequent maximal HIF-1 and HIF-2 stabilization, show profound cholesterol-accumulating effects, interfering with oxygen sensors PHD1 and PHD2 upstream of HIFs skews the system to the opposite phenotype. Thus PHD isoforms have a differential and partially HIF-independent mode of action on cholesterol metabolism. 
Mechanistically, we could show enhanced trans-intestinal cholesterol excretion (TICE) to underlie the phenotype in PHD1 deficient mice (see Figure 4), while the mechanism in PHD2 hypomorphic mice remains obscure.

A

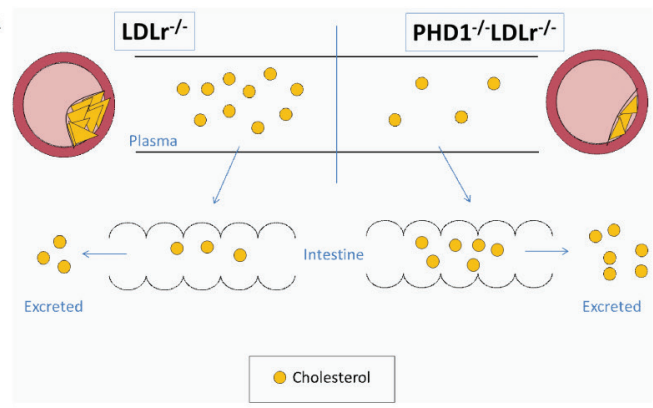

B

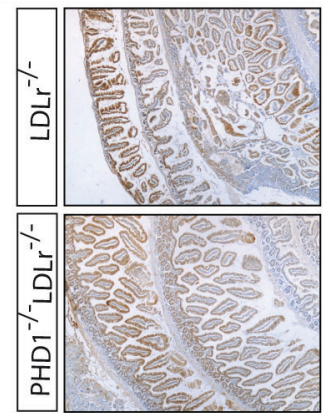

Figure 4:

A. Proposed processes involved in cholesterol-lowering and atheroprotection seen in PHD1 deficient mice. B. pimonidazole staining on swiss-roles of $\mathrm{LDLr}{ }^{-/}$and PHD1- LDL ${ }^{-/}$intestines.

TICE is a complex process and its regulation is far from clear. Looking at the intestinal epithelium lining, PHD1 deficient mice did not show a difference in pimonidazole staining (Figure 4) or general morphology (chapter 5). TICE has been described as an active transport process, with medium oxygenation enhancing TICE in an ex vivo TICE model (103). While this suggests a link to oxygen consumption in PHD1 deficient mice, the ex vivo system has major drawbacks in its translation to the in vivo situation and results from the ex vivo system remain to be confirmed. As an indication of its intestinal function, PHD1 deficiency has been described to enhance epithelial barrier function and prevent colitis (3). Also DMOG and FG4497, which preferentially inhibit PHD2, showed similar effects on intestinal epithelial cell function $(104,105)$. However, whether improved intestinal barrier function is linked to cholesterol uptake or TICE is unknown.

More relevantly, bile composition might affect TICE efficiency (reviewed in (106)). Using NPC1L1 transgenic mice, with enhanced NPC1L1 expression in the liver, reduced biliary cholesterol excretion was shown to promote TICE. Also in our hands, biliary cholesterol excretion was decreased in PHD1 deficient mice (chapter 5) and bile acid composition in bile was shifted towards hydrophilic chenodeoxycholate (CDCA) - derived bile acids (Figure 5A). However, a direct molecular link between bile acid composition and TICE induction as well as PHD1 deficiency and bile composition remains to be shown. Also, the gut microbiota has recently been shown to reduce taurobeta-muricholic acid (T $\beta$-MCA) in bile, a naturally occurring FXR antagonist (107). 
Biliary T $\beta$-MCA levels were actually enhanced in PHD1 deficient mice as compared to their controls (Figure 5B), potentially suggesting altered microbiota in PHD1 deficient mice. In line with T $\beta$-MCA, FXR target genes such as SR-B1 and ABCG5/8 were upregulated in the PHD1 deficiency livers (chapter 5, Figure 5C). Interestingly, hepatic FXR $\alpha 2$ over-expression has recently been shown to promote TICE (108), though the phenotype did not completely match our phenotype. For example, FXR $\alpha 2$ transduction did not affect intestinal cholesterol absorption, which was increased in PHD1 deficient mice (chapter 5). Also, other FXR target genes, such as SREBP1, Cy7A1 and CYP27A1 were not affected in PHD1 deficient mice. Also FXR transcription itself was not altered. This partial activation might be related to presence of co-activators and repressors as well as hetero-dimerization partners (FXR/RXR) (109). Thus, FXR might present a valid candidate in PHD1 mediated cholesterol lowering.

A

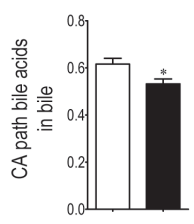

B

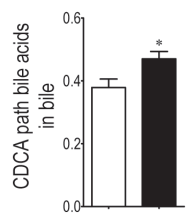

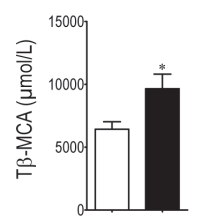

C

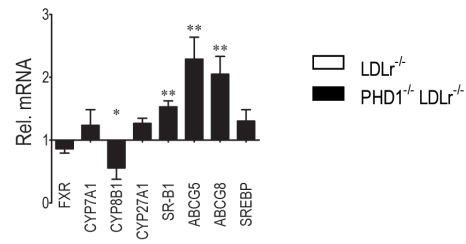

Figure 5: Changes in bile acid regulation in PHD1 deficient mice.

A. Switch in bile composition from cholic acid (CA) towards chenodeoxycholic acid (CDCA). B. T $\beta$-MCA levels are elevated in the bile of PHD1 deficient mice. C. Hepatic FXR target genes are differentially regulated in PHD1 deficient mice. Data is presented relative to expression in LDLr/- mice.

The presence of TICE has recently also been suggested in humans intestines ex vivo (103). Additional circumstantial evidence for TICE comes from looking at the whole body cholesterol balance (figure 6). Overall, non-biliary cholesterol excretion might present a valid new target in reducing plasma cholesterol levels and protecting from atherosclerosis progression. In fact, enhancing biliary cholesterol excretion as in traditional HDL-mediated RCT, might not be desirable as it promotes cholesterol gallstone disease $(110,111)$. 


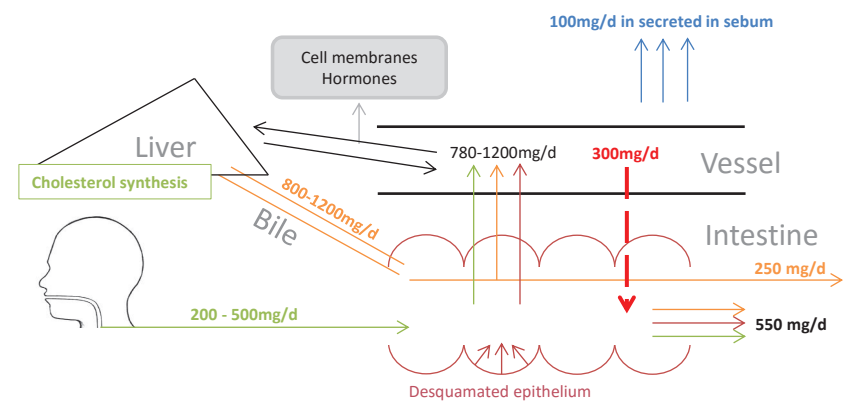

Figure 6: Calculation of TICE (in red) in humans.

Although there are inter-individual differences, a dietary cholesterol intake of $500 \mathrm{mg} / \mathrm{can}$ be estimated. With a biliary cholesterol excretion of $1200 \mathrm{mg} / d$ and $1200 \mathrm{mg} / d$ being reabsorbed, this means that a daily neutral sterol excretion in feces of $800 \mathrm{mg} / \mathrm{d}$ exceeds cholesterol intake by $300 \mathrm{mg} / \mathrm{d}$. While desquamated epithelium might contribute some of the fecal neutral sterols, there must be an alternative route of cholesterol excretion (in red) from plasma into the feces also in bumans (112). Additionally, cholesterol is used for cell membranes and as precursor for hormones and vitamin D (113), and cholesterol synthesis is required to fulfill the daily cholesterol need.

In conclusion, most evidence points towards increased plasma cholesterol levels upon systemic hypoxia. In line with that whole body PHD3 deficiency promoted hypercholesterolemia. Nevertheless, manipulation of hypoxic signaling in mice deficient in the major PHD isoforms, PHD1 and PHD2, led to reduced plasma cholesterol levels. These data suggest differences between PHD deficiency-mediated hypoxic signaling as opposed to systemic hypoxia or sole HIF signaling in plasma cholesterol level regulation.

\section{PHDS IN OBESITY AND DIABETES}

Next to cholesterol metabolism, PHDs have recently been studied in diet-induced obesity and diabetes models. Postnatal whole body PHD2 deficiency could attenuate high fat diet-induced apoptosis and cardiac dysfunction (114), and also adipocytespecific PHD2 deletion conferred resistance to high fat diet-induced obesity. Mice showed improved glucose tolerance and insulin resistance due to uncoupling protein 1 (UCP-1) mediated brown adipose tissue (BAT) activation (115). In line, Rahtu-Korpela et al. show reduced adipocyte size in a hypomorphic model of PHD2 deletion, with lowered plasma cholesterol levels and improved glucose and insulin tolerance (57). The authors describe no difference in BAT activation, based on unchanged BAT UCP-1 mRNA expression. However, it has been realized that mRNA expression levels of UCP1 have weak association with BAT activation and thermogenesis and in fact, protein levels have to be analyzed to allow conclusions about brown adipose tissue activation 
(116). Thus, enhanced BAT activation might still underlie the improved glucose tolerance and insulin resistance seen in PHD2 hypomorphic mice. Similarly, PHD1 deficiency reduced adiposity and plasma glucose levels and improved glucose intolerance (figure 7), an effect that could potentially also be mediated by UCP-1 mediated BAT activation. Indeed, mice lacking HIF- $2 \alpha$ specifically in white and brown adipocytes present with the reverse phenotype: increased weight gain upon diet induced obesity, insulin resistance and glycose intolerance. Also these mice show reduced BAT UCP-1 content and enhanced adipocyte size (117). Fat-tissue specific HIF-2 $\alpha$ in PHD1 deficient mice would give insights into whether improved glucose tolerance is HIF-2 $\alpha$ dependent in our model. Also, specifically targeting HIF- $\alpha$ could be shown to improve glucose tolerance and energy expenditure in mice (118). As a major drawback of these murine adiposity studies, however, mice show rapid adipose tissue expansion during high fat diet, with associated hypoxia and lack of adequate neovascularization. This is not necessarily true for human slowly growing adipose tissue expansion, as critically reviewed in (119). Thus the translation of interfering with hypoxic signaling in humans remains to be shown and should be monitored carefully.

For PHD3, only one study has been published so far, where acute hepatic PHD3 deletion improved insulin sensitivity and ameliorated diabetes by stabilizing HIF- $2 \alpha$, with subsequent insulin receptor substrate-2 (Irs2) induction (120). Irs2 in turn is known to promote insulin sensitivity (121). Interestingly, both HIF-2 $\alpha$ and Irs 2 were required for the improved insulin resistance, for the first time linking hypoxic signaling with insulin signaling (120). As mentioned, hepatic HIF-2 $\alpha$ stabilization has recently been shown to profoundly increase plasma cholesterol levels (82). However plasma cholesterol levels were not investigated in the PHD3 diabetes study and might also show different results due to different diets.
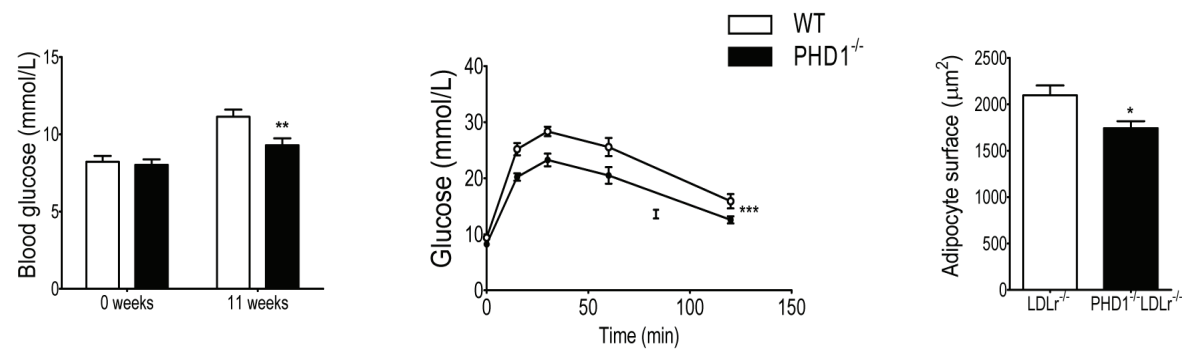

Figure 7:

PHD1 deficiency reduces blood glucose levels and glucose intolerance upon high fat diet (14 weeks, 60\%kcal\%fat). Adipocyte size in PHD1--LDLr/- mice upon high cholesterol diet is also reduced. (Adjusted from Marsch et al. EHJ, accepted) 
To conclude, whole body PHD inhibition seems to improve glucose tolerance, reduce adiposity and might affect diabetes, independent of isoform inhibition in mice. This is in contrast to differential isoform effects on atherosclerosis and hypercholesterolemia investigated in this disseration. Yet, the processes involved in improving glucose tolerance might differ between the PHD isoforms.

\section{DEVELOPMENT OF SPECIFIC PHDI INHIBITORS}

Cardiovascular diseases remain the main cause of death globally (122). Although the incidence of myocardial infarctions decreases in the western world due to life style improvements or other risk-factor modification as well as therapeutic advances (123), coronary event numbers are rising in the low-to-middle income countries (124). Indeed $82 \%$ of all non-communicable diseases (CVD, cancer, chronic respiratory diseases and diabetes) occur in low and middle income countries of which $37 \%$ are caused by CVDs (122). On the one hand, this shows that our current strategies are effective in lowering CVD in the western world, though they have not yet reached the low-to-middle income countries. On the other hand, we need additional strategies to treat the remaining still impressive number of CVD patients.

As summarized in chapter 1 , atherosclerosis is considered a cholesterol-driven chronic inflammatory disease. Current therapies for atherosclerosis involve surgical removal of the plaque and risk factor management mainly, with anti-inflammatory agents undergoing clinical trials at the moment. While the effectiveness of the latter remains to be shown, epidemiological evidence mainly points towards risk factor management as a good therapeutic strategy. Indeed, diabetes increases coronary atherosclerosis risk by 3-5 fold $(125,126)$ and hypertensive patients show a $27 \%$ increase in myocardial infarctions (MI) and $42 \%$ increase in stroke compared to healthy controls (127). Anti-hypertensives in turn reduce stroke risk by $42 \%$ and MI risk by $14 \%$ (128).

More strikingly, the evidence linking serum cholesterol levels with CVD is unequivocal. Individuals with familial hypercholesterolemia $(\mathrm{FH})$ due to a mutation in the LDLr gene, present a 2-3 fold increase in cholesterol levels, solely attributable to LDL-C elevation. Of these individuals, $85 \%$ will have experienced a MI by the age of 60 (129). These data show a strong influence of cholesterol and particularly LDL-C elevation on atherosclerotic disease risk. Indeed, statins can lower plasma LDL-C levels by $28 \%$ and reduce coronary events by $29 \%$ (130). However, also statins are not a wonder drug. Indeed, only fewer than 1 in 3 adults in the US with elevated LDL-C levels achieve desired LDL-C lowering with statins (131). This is partially due to wrong dosing of 
statins, but also statin resistance $(132,133)$ and even intolerance has been described (134). Thus, new or additional approaches in cholesterol -lowering are desirable. As an example of a new approach, PCSK-9 targeting is currently explored in clinical trials. A recent metaanalysis shows a tremendous $60 \%$ reduction in LDL cholesterol and reduced incidence of all-cause mortality (135-137).

Although these are extremely promising data, new cholesterol-lowering approaches present valid strategies to reduce atherosclerotic disease risk. In this thesis, we describe the potential of cholesterol-lowering via PHD1 inhibition. Mechanistically, the process was independent of cholesterol synthesis or LDLr effects (chapter 5). We therefore envision PHD1 inhibition as a potential add-on novel therapy in hypercholesterolemia treatment. Additionally, we could show a metabolically favorable phenotype in PHD1 deficiency, which showed reduced hypercholesterolemia to approximately the same extend as statins, circulating immune cells, atherosclerotic plaque development (chapter 5) and glucose intolerance (chapter 7). Thus, PHD1 inhibition might have additional applications in metabolic disease per se.

PHD inhibitors are currently undergoing clinical trials as erythropoiesis-stimulating drugs for anemia patients (www.clinicaltrials.org). These data show the drugability of PHDs and shortterm safety of interfering with PHDs systemically. However, inhibitors in clinical trials mainly target PHD2. As a note of caution on PHD2 inhibition in cardiovascular disease though, there are also reports on cardiac-specific PHD2 inactivation causing cardiomyopathy over time (138). The premature lethality of PHD2 whole body deficient mice and development of dilated cardiomyopathy in tamoxifen-induced PHD2 deficiency (injections near term and 3 weeks after birth) (139) raise additional concerns of PHD2 inhibition in CVD. Also, PHD2 deficiency is a major inducer of HIF-dependent erythropoiesis (140). While PHD2 inhibition is thus beneficial in anemia patients (see chapter 1), raising erythropoiesis and elevating hematocrit in subjects with normal erythrocyte counts, could potentially thwart the beneficial cardiovascular effects by possible effects on thrombosis (141-143). PHD2 inhibition might thus not be preferable. PHD3 is equally unattractive as a therapeutic target, as PHD3 deficiency actually enhanced plasma cholesterol levels (chapter 6).

As an indication on safety of targeting PHD1 specifically, polymorphisms and disease outcome can be checked. This will give additional insight into the exact function of protein domains. Indeed, one functional polymorphism has been described for PHD1. A polymorphism in the PHD1 proximal promoter (rs10680577) resulted in enhanced PHD1 protein expression and simultaneous RERT-lncRNA expression (a long noncoding RNA whose sequence overlaps with Ras-related GTP-binding protein 4b (RAB4B) and EGLN2). While the mechanism of PHD1 overexpression in this case is unknown the authors suggest 
that RERT-lncRNA affects recruitment of general transcription factors onto the promoter of PHD1 (144). This polymorphism increased risk for gastric cancer (145), non-small lung cancer (146) and hepatocellular carcinoma (144) in Chinese populations. Thus even in these cancers, PHD1 inhibition might be favorable. However, cancer cells have to be considered with caution. Although PHDs might be overexpressed in tumors, this does not necessarily imply more HIF degradation in tumor tissue. In fact, fumarate hydratase and succinate dehydrogenase mutations, resulting in succinate and fumarate accumulation in tumors have been described. In turn accumulating succinate and fumarate can reduce PHD activity, thereby resulting in HIF stabilization $(147,148)$. Likewise, ectopic expression of PHD1 in a mouse xenograft colon carcinoma model inhibited tumor growth and microvessel growth by inhibiting HIF-1 (149). In line with those results, an additional germ-line mutation has been identified in one patient with pheochromocytoma/paraganglioma-polycythemia. The mutation was located in the $\mathrm{C}$ terminus of the protein and resulted in reduced stability of the PHD1 protein with a 5 fold reduced half-life. Also, HIF-1 and HIF-2 were stabilized in tumor tissue of this patient and EPO responsiveness was increased (150). Obviously, this case-study does not allow any conclusions on erythropoiesis and cholesterol levels. Moreover, we and others do not find any erythropoiesis effects of PHD1 inhibition in murine models (chapter 5 (45)). Nevertheless, the human situation should be monitored carefully, in particular for cancer patients in general.

PHD inhibitors undergoing clinical trials at the moment are selective for PHD2, partially due to the relative abundance of PHD2 and partially based on affinity selection, but specific inhibitors for the other PHD isoenzymes are still lacking. In silico drug screening using PHD specific 3D structures might provide a tool for generating specific inhibitors. Crystal structures for in silico screening but also structure-function analysis are available for FIH (151-153) and truncated versions of PHD2 (154-157). For PHD2 structures, Christopher Schofield has pioneered the field. His group could show that only truncated PHD2 proteins (lack of $\mathrm{N}$ terminus) render highly active protein and thus they established a series of $3 \mathrm{D}$ structures of the $\mathrm{C}$ terminus of PHD2. In those structures, they also identified a mobile region within the protein that isolated the active site and stabilizes the PHD2/ $\mathrm{Fe}^{2+} / 2 \mathrm{OG}$ complex (154). Based on these structures, in silico screening for small molecule inhibitors of PHD2 has been successfully executed (158).

The difficulty of generation a 3D structure of the whole PHD protein has complicated the generation of PHD isoform specific inhibitors. Thus, we are exploiting the potential of in silico modeling of the PHD1 structure derived from PHD2 structures. This is 
relatively reliable for the $\mathrm{C}$ terminus because of the great homology between the PHD isoforms and the availability of the $\mathrm{C}$ terminal structure of the PHD2 protein. As a result of the C-terminal homology, the $\mathrm{N}$ terminus might actually confer substrate specificity. We have additionally made a de novo $\mathrm{N}$ terminal model, however, de novo modelling is generally considered less reliable than 3D structure based design (Figure 8).

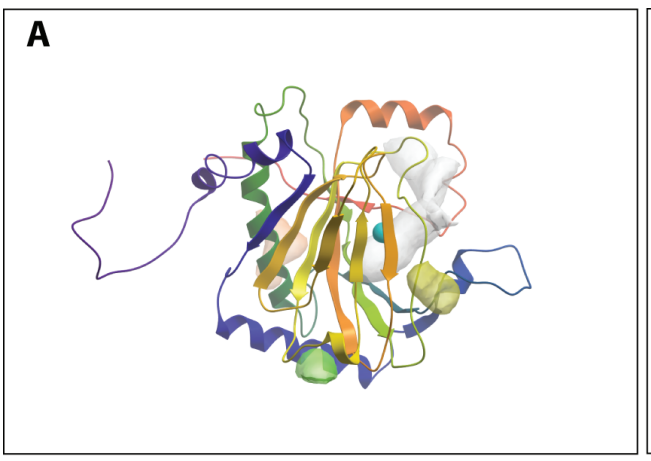

\section{B}

Figure 8: PHD1 3D structure with druggable pockets.

A. 3D structure PHD1 derived from PHD2 structures (monv.rcsb.org) white, yellow and green indicate druggable pockets identified with ICM-Pocket Finder and QSiteFinder. B. De novo model of PHD1 N terminus with in blue and red druggable pockets *Results obtained in collaboration with Dr. Gerry Nicolaes, Biochemistry Department, Maastricht University.

Simultaneously, we are making use of state-of-the-art expression systems to produce the whole PHD1 recombinant protein. Insect cell-expression systems have been successfully used to express whole PHD1, 2 and 3 proteins, but protein yield from these systems was not sufficient to allow NMR or X-ray analysis. To improve yields, we have optimized the sequence of the human PHD1 gene (OptimumGeneTM Codon Optimalization Analysis, Genscript) for various parameters critical to the efficiency of gene expression in E.coli (e.g. codon usage bias, GC content and additional cryptic splicing sites). Next, we used the optimized sequence for cloning into a Strep-tag system for one step purifications (159). Expression experiments are still ongoing, but this system will allow fast and one-step purification of our expressed protein of interest, being strep-tagged at the $\mathrm{N}$ terminus.

With this approach we aim to produce enough protein for NMR 3D structure analysis of the whole PHD1 protein and in silico drug screening (by virtual compound library screening). Additionally, truncated versions of the protein can then be investigated for structure and function. In fact, the recombinant protein will allow functional studies e.g. identification of potentially novel binding or hydroxylation partners. 
Overall, PHD1 might present a novel and feasible drug target in hypercholesterolemia, atherosclerosis and metabolic disease. In this dissertation, we have identified a novel role of PHD1 in cholesterol-lowering and attempted first steps into translating this finding into a drug target. In particular, statin-resistant might present an appropriate patient population for PHD1 inhibitors. PHD1 inhibition seems to work independently of cholesterol synthesis and LDLr manipulation and affects TICE instead. Whether this same pathway is also affected in humans, remains to be established. The fact that also in humans, general inhibition of all PHDs by Roxadustat and GSK1278863 cholesterol levels are lowered, however, supports our translational approach of PHD1 inhibition $(101,102)$.

\section{CONCLUDING REMARKS}

In this thesis we showed an atherosclerosis-modulatory function of plaque hypoxia towards and identified a novel role of oxygen sensors in cholesterol metabolism.

Hypoxia represents a complex cluster of reactions occurring in a range of low oxygen concentrations, with amongst others HIF stabilization and subsequent target gene activation. Studying a complex phenomenon as this in an equally as complex and multifactorial disease such as atherosclerosis is challenging. Nevertheless, systemic oxygen therapy seems to alleviate atherosclerotic and cardiovascular disease burden, by reducing hypercholesterolemia, inflammation and hypertension. Seeing the widespread presence of atherosclerotic disease oxygen therapy is however not feasible.

Plaque hypoxia can result from decreased oxygen diffusion into the plaque or enhanced cellular oxygen consumption. Reducing cellular oxygen consumption in PHD1 deficient mice could indeed reduce plaque hypoxia, but a profound cholesterol-lowering effect overruled potential local hypoxia signaling effects of PHD1 deficiency on plaque development. Dietary nitrate supplementation (to reduce cellular oxygen costs) or PHD2 and PHD3 deficiency did not affect plaque hypoxia and these models therefore neither proof nor disproof a causal contribution of cellular oxygen consumption on plaque progression. In contrast, improving oxygen delivery to the plaque by administering systemic carbogen gas, reduced atherosclerotic plaque necrotic core size. Overall, we suggest a modulatory role of plaque hypoxia in atherosclerotic disease.

Aside from hypoxia, we could show that PHDs have cell type- and isoform-specific effects on plaque development and progression and affect different atherosclerotic processes. Similarly, PHD deficiency differentially affected plasma cholesterol levels. 
In contrast, whole body PHD inhibition seems to improve glucose tolerance, reduce adiposity and might affect diabetes, independent of isoform inhibition in models of diet-induced obesity. While, general PHD inhibition has been shown to also lower plasma cholesterol levels in humans, adiposity effects remain to be translated to humans.

Cell type- and isoform-specificity thus has to be considered when investigating PHDs as potential therapeutic targets. In line with that, combinatory inhibition (e.g. PHD1 and PHD3) might present with even more diverse outcomes and potential in translational research. Nonetheless, as our study shows, PHD1 inhibition presents a feasible and attractive drug target in hypercholesterolemia and atherosclerosis alleviation. 


\section{REFERENCES}

1. Gonzalez C. Physiological hypoxia: concept, mechanisms of detection and responses. Proc Physiol Soc. The Physiological Society; 2003; Physiol .

2. Carreau A, El Hafny-Rahbi B, Matejuk A, Grillon C, Kieda C. Why is the partial oxygen pressure of human tissues a crucial parameter? Small molecules and hypoxia. J Cell Mol Med. 2011 Jun;15(6):1239-53.

3. Karhausen J, Furuta GT, Tomaszewski JE, Johnson RS, Colgan SP, Haase VH. Epithelial hypoxia-inducible factor-1 is protective in murine experimental colitis. J Clin Invest. American Society for Clinical Investigation; 2004 Oct 15;114(8):1098-106.

4. Brezis M, Rosen S. Hypoxia of the renal medulla--its implications for disease. N Engl J Med. 1995 Mar 9;332(10):647-55.

5. Raleigh JA, Koch CJ. Importance of thiols in the reductive binding of 2-nitroimidazoles to macromolecules. Biochem Pharmacol. 1990 Dec;40(11):2457-64.

6. Pasarica M, Sereda OR, Redman LM, Albarado DC, Hymel DT, Roan LE, et al. Reduced Adipose Tissue Oxygenation in Human Obesity: Evidence for Rarefaction, Macrophage Chemotaxis, and Inflammation Without an Angiogenic Response. Diabetes. 2008 Dec 15;58(3):718-25.

7. Goossens GH, Bizzarri A, Venteclef N, Essers Y, Cleutjens JP, Konings E, et al. Increased adipose tissue oxygen tension in obese compared with lean men is accompanied by insulin resistance, impaired adipose tissue capillarization, and inflammation. Circulation. 2011 Jul 5;124(1):67-76.

8. Jurrus ER, Weiss HS. In vitro tissue oxygen tensions in the rabbit aortic arch. Atherosclerosis. 1977 Nov;28(3):223-32.

9. Zemplenyi T, Crawford DW, Cole MA. Adaptation to arterial wall hypoxia demonstrated in vivo with oxygen microcathodes. Atherosclerosis. 1989 Apr;76(2-3):173-9.

10. Höckel M, Vaupel P. Biological consequences of tumor hypoxia. Semin Oncol. 2001 Apr;28(2 Suppl 8):36-41.

11. Leppänen O, Björnheden T, Evaldsson M, Borén J, Wiklund O, Levin M. ATP depletion in macrophages in the core of advanced rabbit atherosclerotic plaques in vivo. Atherosclerosis. 2006 Oct;188(2):323-30.

12. Naghavi M, John R, Naguib S, Siadaty MS, Grasu R, Kurian KC, et al. pH Heterogeneity of human and rabbit atherosclerotic plaques; a new insight into detection of vulnerable plaque. Atherosclerosis. 2002;164(1):27-35.

13. ALTSCHUL R, HERMAN IH. Influence of oxygen inhalation on cholesterol metabolism. Arch Biochem Biophys. 1954 Jul;51(1):308-9.

14. Vesselinovitch D, Wissler RW, Fisher-Dzoga K, Hughes R, Dubien L. Regression of atherosclerosis in rabbits. I. Treatment with low-fat diet, hyperoxia and hypolipidemic agents. Atherosclerosis. Jan;19(2):259-75.

15. Castro-Grattoni AL, Alvarez R, Torres M, Farré R, Montserrat JM, Dalmases M, et al. Intermittent Hypoxia-Induced Cardiovascular Remodeling is Reversed by Normoxia in A Mouse Model of Sleep Apnea. Chest. 2016 Jan 13;

16. Buchner NJ, Quack I, Woznowski M, Stahle C, Wenzel U, Rump LC. Microvascular endothelial dysfunction in obstructive sleep apnea is caused by oxidative stress and improved by continuous positive airway pressure therapy. Respiration. 2011/02/12 ed. 2011;82(5):409-17.

17. Drager LF, Bortolotto LA, Figueiredo AC, Krieger EM, Lorenzi GF. Effects of continuous positive airway pressure on early signs of atherosclerosis in obstructive sleep apnea. Am J Respir Crit Care Med. 2007 Oct 1;176(7):706-12.

18. Leech JA, Onal E, Lopata M. Nasal CPAP continues to improve sleep-disordered breathing and daytime oxygenation over long-term follow-up of occlusive sleep apnea syndrome. Chest. 1992 Dec;102(6):1651-5. 
19. Kudchodkar BJ, Wilson J, Lacko A, Dory L. Hyperbaric oxygen reduces the progression and accelerates the regression of atherosclerosis in rabbits. Arterioscler Thromb Vasc Biol. 2000 Jun;20(6):1637-43.

20. Kudchodkar BJ, Pierce A, Dory L. Chronic hyperbaric oxygen treatment elicits an antioxidant response and attenuates atherosclerosis in apoE knockout mice. Atherosclerosis. 2007 Jul;193(1):28-35.

21. Kudchodkar B, Jones H, Simecka J, Dory L. Hyperbaric oxygen treatment attenuates the proinflammatory and immune responses in apolipoprotein E knockout mice. Clin Immunol. 2008/07/04 ed. 2008;128(3):435-41.

22. Thorp E, Cui D, Schrijvers DM, Kuriakose G, Tabas I. Mertk receptor mutation reduces efferocytosis efficiency and promotes apoptotic cell accumulation and plaque necrosis in atherosclerotic lesions of apoe-/- mice. Arterioscler Thromb Vasc Biol. 2008 Aug;28(8):1421-8.

23. Torres Filho IP, Leunig M, Yuan F, Intaglietta M, Jain RK. Noninvasive measurement of microvascular and interstitial oxygen profiles in a human tumor in SCID mice. Proc Natl Acad Sci U S A. 1994 Mar 15;91(6):2081-5.

24. Raveh O, Pinchuk I, Fainaru M, Lichtenberg D. Oxygen availability as a possible limiting factor in LDL oxidation. Free Radic Res. 2002 Oct;36(10):1109-14.

25. Chisolm GM, Hazen SL, Fox PL, Cathcart MK. The Oxidation of Lipoproteins by Monocytes-Macrophages: BIOCHEMICAL AND BIOLOGICAL MECHANISMS. J Biol Chem. 1999 Sep 10;274(37):25959-62.

26. O'Neill LAJ, Hardie DG. Metabolism of inflammation limited by AMPK and pseudostarvation. Nature. 2013 Jan 17;493(7432):346-55.

27. Hard GC. Some biochemical aspects of the immune macrophage. Br J Exp Pathol. 1970 Feb;51(1):97-105.

28. Tawakol A, Singh P, Mojena M, Pimentel-Santillana M, Emami H, MacNabb M, et al. HIF$1 \alpha$ and PFKFB3 Mediate a Tight Relationship Between Proinflammatory Activation and Anerobic Metabolism in Atherosclerotic Macrophages. Arterioscler Thromb Vasc Biol. 2015 Jun 1;35(6):146371.

29. Stöger JL, Gijbels MJJ, van der Velden S, Manca M, van der Loos CM, Biessen EAL, et al. Distribution of macrophage polarization markers in human atherosclerosis. Atherosclerosis. 2012 Dec;225(2):461-8.

30. Sluimer JC, Gasc J-M, van Wanroij JL, Kisters N, Groeneweg M, Sollewijn Gelpke MD, et al. Hypoxia, hypoxia-inducible transcription factor, and macrophages in human atherosclerotic plaques are correlated with intraplaque angiogenesis. J Am Coll Cardiol. 2008 Apr 1;51(13):1258-65.

31. Colgan SP, Eltzschig HK. Adenosine and hypoxia-inducible factor signaling in intestinal injury and recovery. Annu Rev Physiol. Annual Reviews; 2012 Jan 15;74:153-75.

32. Eckle T, Brodsky K, Bonney M, Packard T, Han J, Borchers CH, et al. HIF1A reduces acute lung injury by optimizing carbohydrate metabolism in the alveolar epithelium. PLoS Biol. Public Library of Science; 2013 Sep 24;11(9):e1001665.

33. Eckle T, Hartmann K, Bonney S, Reithel S, Mittelbronn M, Walker LA, et al. Adora2b-elicited Per2 stabilization promotes a HIF-dependent metabolic switch crucial for myocardial adaptation to ischemia. Nat Med. Nature Publishing Group, a division of Macmillan Publishers Limited. All Rights Reserved.; 2012 May;18(5):774-82.

34. Eltzschig HK, Ibla JC, Furuta GT, Leonard MO, Jacobson KA, Enjyoji K, et al. Coordinated adenine nucleotide phosphohydrolysis and nucleoside signaling in posthypoxic endothelium: role of ectonucleotidases and adenosine A2B receptors. J Exp Med. 2003 Sep 1;198(5):783-96.

35. Eltzschig HK, Abdulla P, Hoffman E, Hamilton KE, Daniels D, Schönfeld C, et al. HIF-1dependent repression of equilibrative nucleoside transporter (ENT) in hypoxia. J Exp Med. 2005 Dec 5;202(11):1493-505. 
36. Thompson LF, Eltzschig HK, Ibla JC, Van De Wiele CJ, Resta R, Morote-Garcia JC, et al. Crucial role for ecto-5'-nucleotidase (CD73) in vascular leakage during hypoxia. J Exp Med. 2004 Dec 6;200(11):1395-405.

37. Eckle T, Faigle M, Grenz A, Laucher S, Thompson LF, Eltzschig HK. A2B adenosine receptor dampens hypoxia-induced vascular leak. Blood. 2008 Feb 15;111(4):2024-35.

38. Rosenberger P, Schwab JM, Mirakaj V, Masekowsky E, Mager A, Morote-Garcia JC, et al. Hypoxia-inducible factor-dependent induction of netrin-1 dampens inflammation caused by hypoxia. Nat Immunol. 2009 Feb;10(2):195-202.

39. Larsen FJ, Schiffer TA, Borniquel S, Sahlin K, Ekblom B, Lundberg JO, et al. Dietary inorganic nitrate improves mitochondrial efficiency in humans. Cell Metab. 2011 Feb 2;13(2):149-59.

40. Larsen FJ, Weitzberg E, Lundberg JO, Ekblom B. Effects of dietary nitrate on oxygen cost during exercise. Acta Physiol (Oxf). 2007 Sep;191(1):59-66.

41. Larsen FJ, Weitzberg E, Lundberg JO, Ekblom B. Dietary nitrate reduces maximal oxygen consumption while maintaining work performance in maximal exercise. Free Radic Biol Med. 2010 Jan 15;48(2):342-7.

42. Jädert C, Petersson J, Massena S, Ahl D, Grapensparr L, Holm L, et al. Decreased leukocyte recruitment by inorganic nitrate and nitrite in microvascular inflammation and NSAID-induced intestinal injury. Free Radic Biol Med. 2012 Feb 1;52(3):683-92.

43. Jädert C, Phillipson M, Holm L, Lundberg JO, Borniquel S. Preventive and therapeutic effects of nitrite supplementation in experimental inflammatory bowel disease. Redox Biol. 2014 Jan;2:73-81. 44. Semenza GL. Targeting HIF-1 for cancer therapy. Nat Rev Cancer. 2003 Oct;3(10):721-32.

45. Aragonés J, Schneider M, Van Geyte K, Fraisl P, Dresselaers T, Mazzone M, et al. Deficiency or inhibition of oxygen sensor Phd1 induces hypoxia tolerance by reprogramming basal metabolism. Nat Genet. 2008 Feb;40(2):170-80.

46. McCarthy MJ, Loftus IM, Thompson MM, Jones L, London NJM, Bell PRF, et al. Angiogenesis and the atherosclerotic carotid plaque: An association between symptomatology and plaque morphology. J Vasc Surg. 1999 Aug;30(2):261-8.

47. Burke AP, Farb A, Malcom GT, Liang Y, Smialek JE, Virmani R. Plaque Rupture and Sudden Death Related to Exertion in Men With Coronary Artery Disease. JAMA. American Medical Association; 1999 Mar 10;281(10):921.

48. Sluimer JC, Kolodgie FD, Bijnens APJJ, Maxfield K, Pacheco E, Kutys B, et al. Thin-walled microvessels in human coronary atherosclerotic plaques show incomplete endothelial junctions relevance of compromised structural integrity for intraplaque microvascular leakage. J Am Coll Cardiol. 2009 Apr 28;53(17):1517-27.

49. Mofidi R, Crotty TB, McCarthy P, Sheehan SJ, Mehigan D, Keaveny T V. Association between plaque instability, angiogenesis and symptomatic carotid occlusive disease. Br J Surg. 2001 Jul;88(7):945-50.

50. Loinard C, Ginouvès A, Vilar J, Cochain C, Zouggari Y, Recalde A, et al. Inhibition of prolyl hydroxylase domain proteins promotes therapeutic revascularization. Circulation. 2009 Jul 7;120(1):50 9 .

51. Khurana R, Simons M, Martin JF, Zachary IC. Role of angiogenesis in cardiovascular disease: a critical appraisal. Circulation. 2005 Sep 20;112(12):1813-24.

52. Moulton KS, Heller E, Konerding MA, Flynn E, Palinski W, Folkman J. Angiogenesis Inhibitors Endostatin or TNP-470 Reduce Intimal Neovascularization and Plaque Growth in Apolipoprotein E Deficient Mice. Circulation. 1999 Apr 6;99(13):1726-32.

53. Moulton KS, Vakili K, Zurakowski D, Soliman M, Butterfield C, Sylvin E, et al. Inhibition of plaque neovascularization reduces macrophage accumulation and progression of advanced atherosclerosis. Proc Natl Acad Sci U S A. 2003 Apr 15;100(8):4736-41.

54. Van der Donckt C, Van Herck JL, Schrijvers DM, Vanhoutte G, Verhoye M, Blockx I, et al. 
Elastin fragmentation in atherosclerotic mice leads to intraplaque neovascularization, plaque rupture, myocardial infarction, stroke, and sudden death. Eur Heart J. 2015 May 1;36(17):1049-58.

55. Al-Mashhadi RH, Sørensen CB, Kragh PM, Christoffersen C, Mortensen MB, Tolbod LP, et al. Familial hypercholesterolemia and atherosclerosis in cloned minipigs created by DNA transposition of a human PCSK9 gain-of-function mutant. Sci Transl Med. 2013 Jan;5(166):166ra1.

56. Rahtu-Korpela L, Määttä J, Dimova EY, Hörkkö S, Gylling H, Walkinshaw G, et al. HypoxiaInducible Factor-Prolyl 4-Hydroxylase-2 Inhibition Protects Against Development of Atherosclerosis. Arterioscler Thromb Vasc Biol. 2016 Feb 4;ATVBAHA.115.307136 - .

57. Rahtu-Korpela L, Karsikas S, Hörkkö S, Blanco Sequeiros R, Lammentausta E, Mäkelä KA, et al. HIF prolyl 4-hydroxylase- 2 inhibition improves glucose and lipid metabolism and protects against obesity and metabolic dysfunction. Diabetes. 2014 Oct;63(10):3324-33.

58. Celletti FL, Waugh JM, Amabile PG, Brendolan A, Hilfiker PR, Dake MD. Vascular endothelial growth factor enhances atherosclerotic plaque progression. Nat Med. 2001;7(4):425-9.

59. Drinane M, Mollmark J, Zagorchev L, Moodie K, Sun B, Hall A, et al. The antiangiogenic activity of rPAI-1(23) inhibits vasa vasorum and growth of atherosclerotic plaque. Circ Res. 2009 Feb;104(3):337-45.

60. Haghighat A, Weiss D, Whalin MK, Cowan DP, Taylor WR. Granulocyte colonystimulating factor and granulocyte macrophage colony-stimulating factor exacerbate atherosclerosis in apolipoprotein E-deficient mice. Circulation. 2007 Apr;115(15):2049-54.

61. Inoue M, Ishida T, Yasuda T, Toh R, Hara T, Cangara HM, et al. Endothelial cell-selective adhesion molecule modulates atherosclerosis through plaque angiogenesis and monocyte-endothelial interaction. Microvasc Res. Elsevier Inc.; 2010 Sep;80(2):179-87.

62. Luttun A, Tjwa M, Moons L, Wu Y, Angelillo-Scherrer A, Liao F, et al. Revascularization of ischemic tissues by PlGF treatment, and inhibition of tumor angiogenesis, arthritis and atherosclerosis by anti-Flt1. Nat Med. 2002;8(8):831-40.

63. Leppanen P, Koota S, Kholova I, Koponen J, Fieber C, Eriksson U, et al. Gene transfers of vascular endothelial growth factor-A, vascular endothelial growth factor-B, vascular endothelial growth factor-C, and vascular endothelial growth factor-D have no effects on atherosclerosis in hypercholesterolemic low-density lipoprotein. Circulation. 2005;112(9):1347-52.

64. Veerman KJ, Venegas-Pino DE, Shi Y, Khan MI, Gerstein HC, Werstuck GH. Hyperglycaemia is associated with impaired vasa vasorum neovascularization and accelerated atherosclerosis in apolipoprotein-E deficient mice. Atherosclerosis. Elsevier Ltd; 2013 Apr;227(2):250-8.

65. Petrovan RJ, Kaplan CD, Reisfeld RA, Curtiss LK. DNA vaccination against VEGF receptor 2 reduces atherosclerosis in LDL receptor-deficient mice. Arter Thromb Vasc Biol. 2007;27(5):1095100.

66. Nakano M, Fukumoto Y, Satoh K, Ito Y, Kagaya Y, Ishii N, et al. OX40 ligand plays an important role in the development of atherosclerosis through vasa vasorum neovascularization. Cardiovasc Res. 2010 Dec;88(3):539-46.

67. Horino Y, Takahashi S, Miura T, Takahashi Y. Prolonged hypoxia accelerates the posttranscriptional process of collagen synthesis in cultured fibroblasts. Life Sci. 2002 Nov 15;71(26):3031-45.

68. Makris EA, Hu JC, Athanasiou KA. Hypoxia-induced collagen crosslinking as a mechanism for enhancing mechanical properties of engineered articular cartilage. Osteoarthritis Cartilage. 2013 Apr;21(4):634-41.

69. Liu Y, Huo Z, Yan B, Lin X, Zhou Z-N, Liang X, et al. Prolyl hydroxylase 3 interacts with Bcl-2 to regulate doxorubicin-induced apoptosis in H9c2 cells. Biochem Biophys Res Commun. 2010 Oct 15;401(2):231-7.

70. Bishop T, Gallagher D, Pascual A, Lygate CA, de Bono JP, Nicholls LG, et al. Abnormal Sympathoadrenal Development and Systemic Hypotension in PHD3-/- Mice. Mol Cell Biol. 2008 Mar 
10;28(10):3386-400.

71. Swain L, Wottawa M, Hillemann A, Beneke A, Odagiri H, Terada K, et al. Prolyl-4hydroxylase domain 3 (PHD3) is a critical terminator for cell survival of macrophages under stress conditions. J Leukoc Biol. 2014 Sep;96(3):365-75.

72. Walmsley SR, Chilvers ER, Thompson AA, Vaughan K, Marriott HM, Parker LC, et al. Prolyl hydroxylase 3 (PHD3) is essential for hypoxic regulation of neutrophilic inflammation in humans and mice. J Clin Invest. 2011 Mar;121(3):1053-63.

73. Watkins H, Farrall M. Genetic susceptibility to coronary artery disease: from promise to progress. Nat Rev Genet. 2006 Mar; 7(3):163-73.

74. Minamishima YA, Moslehi J, Padera RF, Bronson RT, Liao R, Kaelin WG. A feedback loop involving the Phd3 prolyl hydroxylase tunes the mammalian hypoxic response in vivo. Mol Cell Biol. 2009 Nov 1;29(21):5729-41.

75. Stiehl DP, Wirthner R, Köditz J, Spielmann P, Camenisch G, Wenger RH. Increased prolyl 4-hydroxylase domain proteins compensate for decreased oxygen levels. Evidence for an autoregulatory oxygen-sensing system. J Biol Chem. 2006 Aug 18;281(33):23482-91.

76. Appelhoff RJ, Tian Y-M, Raval RR, Turley H, Harris AL, Pugh CW, et al. Differential function of the prolyl hydroxylases PHD1, PHD2, and PHD3 in the regulation of hypoxia-inducible factor. J Biol Chem. 2004 Sep 10;279(37):38458-65.

77. Akhtar S, Hartmann P, Karshovska E, Rinderknecht F-A, Subramanian P, Gremse F, et al. Endothelial Hypoxia-Inducible Factor- $1 \alpha$ Promotes Atherosclerosis and Monocyte Recruitment by Upregulating MicroRNA-19a. Hypertension. 2015 Dec;66(6):1220-6.

78. Christoph M, Ibrahim K, Hesse K, Augstein A, Schmeisser A, Braun-Dullaeus RC, et al. Local inhibition of hypoxia-inducible factor reduces neointima formation after arterial injury in ApoE/- mice. Atherosclerosis. 2014 Apr;233(2):641-7.

79. Ben-Shoshan J, Afek A, Maysel-Auslender S, Barzelay A, Rubinstein A, Keren G, et al. HIF1alpha overexpression and experimental murine atherosclerosis. Arterioscler Thromb Vasc Biol. 2009 May;29(5):665-70.

80. Chaudhari SM, Sluimer JC, Koch M, Theelen TL, Manthey HD, Busch M, et al. Deficiency of HIF1 $\alpha$ in Antigen-Presenting Cells Aggravates Atherosclerosis and Type 1 T-Helper Cell Responses in Mice. Arterioscler Thromb Vasc Biol. 2015 Nov 1;35(11):2316-25.

81. Karshovska E, Zernecke A, Sevilmis G, Millet A, Hristov M, Cohen CD, et al. Expression of HIF-1alpha in injured arteries controls SDF-1alpha mediated neointima formation in apolipoprotein E deficient mice. Arterioscler Thromb Vasc Biol. 2007 Dec;27(12):2540-7.

82. Ramakrishnan SK, Taylor M, Qu A, Ahn S-H, Suresh M V, Raghavendran K, et al. Loss of von Hippel-Lindau protein (VHL) increases systemic cholesterol levels through targeting hypoxiainducible factor $2 \alpha$ and regulation of bile acid homeostasis. Mol Cell Biol. 2014 Apr;34(7):1208-20.

83. Tuckerman JR, Zhao Y, Hewitson KS, Tian Y-M, Pugh CW, Ratcliffe PJ, et al. Determination and comparison of specific activity of the HIF-prolyl hydroxylases. FEBS Lett. 2004 Oct 8;576(12):145-50.

84. Drager LF, Li J, Shin M-K, Reinke C, Aggarwal NR, Jun JC, et al. Intermittent hypoxia inhibits clearance of triglyceride-rich lipoproteins and inactivates adipose lipoprotein lipase in a mouse model of sleep apnoea. Eur Heart J. 2012 Mar;33(6):783-90.

85. Robinson G V, Pepperell JCT, Segal HC, Davies RJO, Stradling JR. Circulating cardiovascular risk factors in obstructive sleep apnoea: data from randomised controlled trials. Thorax. 2004 Sep;59(9):777-82.

86. Comondore VR, Cheema R, Fox J, Butt A, John Mancini GB, Fleetham JA, et al. The impact of CPAP on cardiovascular biomarkers in minimally symptomatic patients with obstructive sleep apnea: a pilot feasibility randomized crossover trial. Lung. Jan;187(1):17-22.

87. Coughlin SR, Mawdsley L, Mugarza JA, Wilding JPH, Calverley PMA. Cardiovascular and 
metabolic effects of CPAP in obese males with OSA. Eur Respir J. 2007 Apr;29(4):720-7.

88. Nenseter MS, Gudmundsen O, Roos N, Maelandsmo G, Drevon CA, Berg T. Role of liver endothelial and Kupffer cells in clearing low density lipoprotein from blood in hypercholesterolemic rabbits. J Lipid Res. 1992 Jun;33(6):867-77.

89. Huang W, Metlakunta A, Dedousis N, Zhang P, Sipula I, Dube JJ, et al. Depletion of liver Kupffer cells prevents the development of diet-induced hepatic steatosis and insulin resistance. Diabetes. 2010 Feb;59(2):347-57.

90. Cuchel M, Rader DJ. Macrophage reverse cholesterol transport: key to the regression of atherosclerosis? Circulation. 2006 May 30;113(21):2548-55.

91. Parathath S, Mick SL, Feig JE, Joaquin V, Grauer L, Habiel DM, et al. Hypoxia is present in murine atherosclerotic plaques and has multiple adverse effects on macrophage lipid metabolism. Circ Res. 2011 Oct 28;109(10):1141-52.

92. Crucet M, Wüst SJA, Spielmann P, Lüscher TF, Wenger RH, Matter CM. Hypoxia enhances lipid uptake in macrophages: role of the scavenger receptors Lox1, SRA, and CD36. Atherosclerosis. 2013 Jul;229(1):110-7.

93. Jiang G, Li T, Qiu Y, Rui Y, Chen W, Lou Y. RNA interference for HIF-1alpha inhibits foam cells formation in vitro. Eur J Pharmacol. 2007 May 21;562(3):183-90.

94. BLOCH K. Biological synthesis of cholesterol. Harvey Lect. Jan;48:68-88.

95. Summons RE, Bradley AS, Jahnke LL, Waldbauer JR. Steroids, triterpenoids and molecular oxygen. Philos Trans R Soc Lond B Biol Sci. 2006 Jun 29;361(1470):951-68.

96. Nguyen AD, McDonald JG, Bruick RK, DeBose-Boyd RA. Hypoxia stimulates degradation of 3-hydroxy-3-methylglutaryl-coenzyme A reductase through accumulation of lanosterol and hypoxiainducible factor-mediated induction of insigs. J Biol Chem. 2007 Sep 14;282(37):27436-46.

97. Ta NL, Seyfried TN. Influence of Serum and Hypoxia on Incorporation of [(14)C]-DGlucose or [(14)C]-L-Glutamine into Lipids and Lactate in Murine Glioblastoma Cells. Lipids. 2015 Dec;50(12):1167-84.

98. Siques P, Brito J, Naveas N, Pulido R, De la Cruz JJ, Mamani M, et al. Plasma and liver lipid profiles in rats exposed to chronic hypobaric hypoxia: changes in metabolic pathways. High Alt Med Biol. 2014 Sep;15(3):388-95.

99. Groen AK, Bloks VW, Verkade H, Kuipers F. Cross-talk between liver and intestine in control of cholesterol and energy homeostasis. Mol Aspects Med. 2014 Jun;37:77-88.

100. Cao R, Zhao X, Li S, Zhou H, Chen W, Ren L, et al. Hypoxia induces dysregulation of lipid metabolism in HepG2 cells via activation of HIF-2 $\alpha$. Cell Physiol Biochem. 2014 Jan;34(5):1427-41.

101. Bakris GL, Yu K-HP, Leong R, Shi W, Lee T, Saikali K, et al. Late-Breaking Orals. J Clin Hypertens. 2012 Jul 13;14(7):487-9.

102. Olson E, Demopoulos L, Haws TF, Hu E, Fang Z, Mahar KM, et al. Short-term treatment with a novel HIF-prolyl hydroxylase inhibitor (GSK1278863) failed to improve measures of performance in subjects with claudication-limited peripheral artery disease. Vasc Med. 2014 Dec 1;19(6):473-82.

103. Le May C, Berger JM, Lespine A, Pillot B, Prieur X, Letessier E, et al. Transintestinal cholesterol excretion is an active metabolic process modulated by PCSK9 and statin involving ABCB1. Arterioscler Thromb Vasc Biol. 2013 Jul 1;33(7):1484-93.

104. Cummins EP, Seeballuck F, Keely SJ, Mangan NE, Callanan JJ, Fallon PG, et al. The hydroxylase inhibitor dimethyloxalylglycine is protective in a murine model of colitis. Gastroenterology. 2008 Jan;134(1):156-65.

105. Robinson A, Keely S, Karhausen J, Gerich ME, Furuta GT, Colgan SP. Mucosal protection by hypoxia-inducible factor prolyl hydroxylase inhibition. Gastroenterology. 2008 Jan;134(1):145-55.

106. Out C, Kuipers F, Groen AK. Bile acids and cholestasis. Gastroenterology. 2013 Feb;144(2):e17-8. 
107. Sayin SI, Wahlström A, Felin J, Jäntti S, Marschall H-U, Bamberg K, et al. Gut microbiota regulates bile acid metabolism by reducing the levels of tauro-beta-muricholic acid, a naturally occurring FXR antagonist. Cell Metab. 2013 Feb 5;17(2):225-35.

108. Boesjes M, Bloks VW, Hageman J, Bos T, van Dijk TH, Havinga R, et al. Hepatic farnesoid $\mathrm{X}$-receptor isoforms $\alpha 2$ and $\alpha 4$ differentially modulate bile salt and lipoprotein metabolism in mice. PLoS One. 2014 Jan;9(12):e115028.

109. Mangelsdorf DJ, Evans RM. The RXR heterodimers and orphan receptors. Cell. 1995 Dec 15;83(6):841-50.

110. Grünhage F, Acalovschi M, Tirziu S, Walier M, Wienker TF, Ciocan A, et al. Increased gallstone risk in humans conferred by common variant of hepatic ATP-binding cassette transporter for cholesterol. Hepatology. 2007 Sep;46(3):793-801.

111. Admirand WH, Small DM. The physicochemical basis of cholesterol gallstone formation in man. J Clin Invest. 1968 May;47(5):1043-52.

112. van der Velde AE, Vrins CLJ, van den Oever K, Kunne C, Oude Elferink RPJ, Kuipers F, et al. Direct intestinal cholesterol secretion contributes significantly to total fecal neutral sterol excretion in mice. Gastroenterology. 2007 Sep;133(3):967-75.

113. Myant NB. The Biology of Cholesterol and Related Steroids. The Biology of Cholesterol and Related Steroids. Elsevier; 1981. 315-337 p.

114. Zeng $\mathrm{H}$, Chen J-X. Conditional knockout of prolyl hydroxylase domain protein 2 attenuates high fat-diet-induced cardiac dysfunction in mice. PLoS One. Public Library of Science; 2014 Jan 29;9(12):e115974.

115. Matsuura H, Ichiki T, Inoue E, Nomura M, Miyazaki R, Hashimoto T, et al. Prolyl hydroxylase domain protein 2 plays a critical role in diet-induced obesity and glucose intolerance. Circulation. 2013 May 28;127(21):2078-87.

116. Nedergaard J, Cannon B. UCP1 mRNA does not produce heat. Biochim Biophys Acta. 2013 May;1831(5):943-9.

117. Garcia Martin R, Rubín de Celis M, Ziogas A, Phieler J, Qin N, Gercken B, et al. Protective role of adipocyte hypoxia-inducible factor-2 in diet-induced obesity in mice. Exp Clin Endocrinol Diabetes. 2014 Mar 5;122(03):P166.

118. Sun K, Halberg N, Khan M, Magalang UJ, Scherer PE. Selective inhibition of hypoxiainducible factor $1 \alpha$ ameliorates adipose tissue dysfunction. Mol Cell Biol. 2013 Mar;33(5):904-17.

119. Goossens GH, Blaak EE. Adipose tissue dysfunction and impaired metabolic health in human obesity: a matter of oxygen? Front Endocrinol (Lausanne). 2015 Jan;6:55.

120. Taniguchi CM, Finger EC, Krieg AJ, Wu C, Diep AN, LaGory EL, et al. Cross-talk between hypoxia and insulin signaling through Phd3 regulates hepatic glucose and lipid metabolism and ameliorates diabetes. Nat Med. 2013 Oct;19(10):1325-30.

121. Taniguchi CM, Ueki K, Kahn R. Complementary roles of IRS-1 and IRS-2 in the hepatic regulation of metabolism. J Clin Invest. 2005 Mar;115(3):718-27.

122. WHO. WHO | Cardiovascular diseases (CVDs). World Health Organization; 2015;

123. Yeh RW, Sidney S, Chandra M, Sorel M, Selby J V., Go AS. Population Trends in the Incidence and Outcomes of Acute Myocardial Infarction. N Engl J Med. 2010 Jun 10;362(23):2155-65.

124. Celermajer DS, Chow CK, Marijon E, Anstey NM, Woo KS. Cardiovascular disease in the developing world: prevalences, patterns, and the potential of early disease detection. J Am Coll Cardiol. 2012 Oct 2;60(14):1207-16.

125. Bierman EL. George Lyman Duff Memorial Lecture. Atherogenesis in diabetes. Arterioscler Thromb. 1992 Jun;12(6):647-56.

126. Pyörälä K, Laakso M, Uusitupa M. Diabetes and atherosclerosis: an epidemiologic view. Diabetes Metab Rev. 1987 Apr;3(2):463-524.

127. MacMahon S, Peto R, Cutler J, Collins R, Sorlie P, Neaton J, et al. Blood pressure, stroke, and 
coronary heart disease. Part 1, Prolonged differences in blood pressure: prospective observational studies corrected for the regression dilution bias. Lancet (London, England). 1990 Mar 31;335(8692):765-74.

128. Collins R, Peto R, MacMahon S, Hebert P, Fiebach NH, Eberlein KA, et al. Blood pressure, stroke, and coronary heart disease. Part 2, Short-term reductions in blood pressure: overview of randomised drug trials in their epidemiological context. Lancet (London, England). 1990 Apr 7;335(8693):827-38.

129. Nair P. Brown and Goldstein: the cholesterol chronicles. Proc Natl Acad Sci U S A. 2013 Sep 10;110(37):14829-32.

130. LaRosa JC, He J, Vupputuri S. Effect of Statins on Risk of Coronary Disease. JAMA. American Medical Association; 1999 Dec 22;282(24):2340.

131. CDC. National Health Report: Leading Causes of Morbidity and Mortality and Associated Behavioral Risk and Protective Factors—United States, 2005-2013. MMWR. 2014;63(04):3-27.

132. Reiner Ž, De Bacquer D, Kotseva K, Prugger C, De Backer G, Wood D. Treatment potential for dyslipidaemia management in patients with coronary heart disease across Europe: findings from the EUROASPIRE III survey. Atherosclerosis. 2013 Dec;231(2):300-7.

133. Reiner $\check{Z}$, Tedeschi-Reiner E. Prevalence and types of persistent dyslipidemia in patients treated with statins. Croat Med J. 2013 Aug;54(4):339-45.

134. Fitchett DH, Hegele RA, Verma S. Cardiology patient page. Statin intolerance. Circulation. 2015 Mar 31;131(13):e389-91.

135. Lipinski MJ, Benedetto U, Escarcega RO, Biondi-Zoccai G, Lhermusier T, Baker NC, et al. The impact of proprotein convertase subtilisin-kexin type 9 serine protease inhibitors on lipid levels and outcomes in patients with primary hypercholesterolaemia: a network meta-analysis. Eur Heart J. 2016 Feb 7;37(6):536-45.

136. European Society of Cardiology. ODYSSEY LONG TERM - Long term results underscore efficacy and safety of alirocum [Internet]. 2014 [cited 2016 Mar 2]. Available from: http://www. escardio.org/The-ESC/Press-Office/Press-releases/Last-5-years/ODYSSEY-LONG-TERM-Longterm-results-underscore-efficacy-and-safety-of-alirocum

137. ClinicalTrials.gov. Monoclonal Antibody Against PCSK9 to Reduce Elevated Low-density Lipoprotein Cholesterol(LDL-C) in Adults Currently NotReceiving DrugTherapy forEasing LipidLevels - Study Results - ClinicalTrials.gov [Internet]. [cited 2016 Feb 5]. Available from: https://clinicaltrials. gov/ct2/show $/$ results $/$ NCT01375777? sect $=$ X70156\&term $=$ PCSK9\&rank=16\#outcome1

138. Moslehi J, Minamishima YA, Shi J, Neuberg D, Charytan DM, Padera RF, et al. Loss of hypoxia-inducible factor prolyl hydroxylase activity in cardiomyocytes phenocopies ischemic cardiomyopathy. Circulation. 2010 Sep 7;122(10):1004-16.

139. Minamishima YA, Moslehi J, Bardeesy N, Cullen D, Bronson RT, Kaelin WG. Somatic inactivation of the PHD2 prolyl hydroxylase causes polycythemia and congestive heart failure. Blood. American Society of Hematology; 2008 Mar 15;111(6):3236-44.

140. Takeda K, Aguila HL, Parikh NS, Li X, Lamothe K, Duan L-J, et al. Regulation of adult erythropoiesis by prolyl hydroxylase domain proteins. Blood. 2008 Mar 15;111(6):3229-35.

141. Holliger P, Prospero T, Winter G. "Diabodies": small bivalent and bispecific antibody fragments. Proc Natl Acad Sci U S A. 1993;90(14):6444-8.

142. Musallam KM, Porter JB, Sfeir PM, Tamim HM, Richards T, Lotta LA, et al. Raised haematocrit concentration and the risk of death and vascular complications after major surgery. $\mathrm{Br} \mathrm{J}$ Surg. 2013 Jul;100(8):1030-6.

143. Phrommintikul A, Haas SJ, Elsik M, Krum H. Mortality and target haemoglobin concentrations in anaemic patients with chronic kidney disease treated with erythropoietin: a metaanalysis. Lancet. Elsevier; 2007 Feb 3;369(9559):381-8.

144. Zhu Z, Gao X, He Y, Zhao H, Yu Q, Jiang D, et al. An insertion/deletion polymorphism within RERT-lncRNA modulates hepatocellular carcinoma risk. Cancer Res. 2012 Dec 1;72(23):6163- 
72.

145. Wang J, Zhang J, Zhou C, Chen L, Yu Q. An insertion/deletion polymorphism within the proximal promoter of EGLN2 is associated with susceptibility for gastric cancer in the Chinese population. Genet Test Mol Biomarkers. 2014 Apr;18(4):269-73.

146. Che J, Jiang D, Zheng Y, Zhu B, Zhang P, Lu D, et al. Polymorphism in PHD1 gene and risk of non-small cell lung cancer in a Chinese population. Tumour Biol. 2014 Sep;35(9):8921-5.

147. King A, Selak MA, Gottlieb E. Succinate dehydrogenase and fumarate hydratase: linking mitochondrial dysfunction and cancer. Oncogene. 2006 Aug 7;25(34):4675-82.

148. Selak MA, Armour SM, MacKenzie ED, Boulahbel H, Watson DG, Mansfield KD, et al. Succinate links TCA cycle dysfunction to oncogenesis by inhibiting HIF- $\alpha$ prolyl hydroxylase. Cancer Cell. 2005 Jan;7(1):77-85.

149. Erez N, Milyavsky M, Eilam R, Shats I, Goldfinger N, Rotter V. Expression of ProlylHydroxylase-1 (PHD1/EGLN2) Suppresses Hypoxia Inducible Factor-1\{alpha\} Activation and Inhibits Tumor Growth. Cancer Res. 2003 Dec 15;63(24):8777-83.

150. Yang C, Zhuang Z, Fliedner SMJ, Shankavaram U, Sun MG, Bullova P, et al. Germ-line PHD1 and PHD2 mutations detected in patients with pheochromocytoma/paraganglioma-polycythemia. J Mol Med (Berl). 2015 Jan;93(1):93-104.

151. Hewitson KS, Liénard BMR, McDonough MA, Clifton IJ, Butler D, Soares AS, et al. Structural and mechanistic studies on the inhibition of the hypoxia-inducible transcription factor hydroxylases by tricarboxylic acid cycle intermediates. J Biol Chem. 2007 Mar 2;282(5):3293-301.

152. McDonough MA, McNeill LA, Tilliet M, Papamicaël CA, Chen Q-Y, Banerji B, et al. Selective inhibition of factor inhibiting hypoxia-inducible factor. J Am Chem Soc. American Chemical Society; 2005 Jun 1;127(21):7680-1.

153. Dann CE, Bruick RK, Deisenhofer J. Structure of factor-inhibiting hypoxia-inducible factor 1: An asparaginyl hydroxylase involved in the hypoxic response pathway. Proc Natl Acad Sci U S A. 2002 Nov 26;99(24):15351-6.

154. Chowdhury R, McDonough MA, Mecinović J, Loenarz C, Flashman E, Hewitson KS, et al. Structural basis for binding of hypoxia-inducible factor to the oxygen-sensing prolyl hydroxylases. Structure. 2009 Jul 15;17(7):981-9.

155. McDonough MA, Li V, Flashman E, Chowdhury R, Mohr C, Liénard BMR, et al. Cellular oxygen sensing: Crystal structure of hypoxia-inducible factor prolyl hydroxylase (PHD2). Proc Natl Acad Sci U S A. 2006 Jun 27;103(26):9814-9.

156. Chowdhury R, Candela-Lena JI, Chan MC, Greenald DJ, Yeoh KK, Tian Y-M, et al. Selective small molecule probes for the hypoxia inducible factor (HIF) prolyl hydroxylases. ACS Chem Biol. 2013 Jul 19;8(7):1488-96.

157. Hong YR, Kim HT, Ro S, Cho JM, Lee SH, Kim IS, et al. Discovery of novel 2-[2-(3-hydroxypyridin-2-yl)-thiazol-4-yl]-acetamide derivatives as HIF prolyl 4-hydroxylase inhibitors; SAR, synthesis and modeling evaluation. Bioorg Med Chem Lett. 2014 Jul 15;24(14):3142-5.

158. Teli MK, Rajanikant GK. Identification of novel potential HIF-prolyl hydroxylase inhibitors by in silico screening. Mol Divers. 2012 Feb;16(1):193-202.

159. Schmidt TGM, Skerra A. The Strep-tag system for one-step purification and high-affinity detection or capturing of proteins. Nat Protoc. 2007 Jan;2(6):1528-35. 



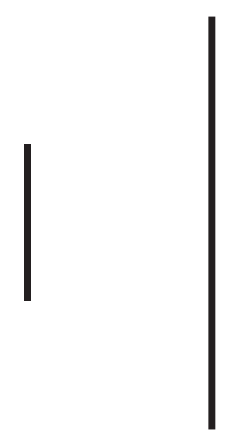

\author{
SUMMARY \\ samenVAtTINg \\ zUSAMMENFAsSUNG
}





\section{SUMMARY}

Atherosclerosis is slowly progressing cholesterol-driven chronic inflammatory disease. It represents the underlying pathology of stroke and myocardial infarction and treatment nowadays mainly consists of surgical removal of the plaque, as well as risk factor management, e.g. serum cholesterol lowering as well as hypertension and diabetes management. A common feature of atherosclerotic plaques is hypoxia, a phenomenon that has been realized but never causally studied with respect to atherosclerotic plaque progression. In this dissertation, it was hypothesized that plaque hypoxia is causal to atherosclerotic disease progression.

First, the literature on hypoxia and inflammation in atherosclerosis was studied (chapter 2). Indeed, the presence of plaque hypoxia had been realized across species and hypoxia had mainly been studied with respect to macrophage function, inflammatory signaling and lipid handling in vitro.

In order to establish a causal relationship, two general approaches to reduce atherosclerotic plaque hypoxia were exploited in this dissertation, namely on the one hand enhancing oxygen availability and on the other hand reducing cellular oxygen consumption within the plaque.

In chapter 3, the onset and localization of plaque hypoxia in murine atherosclerosis was studied. Also the effect of increasing systemic oxygen availability by subjecting mice to carbogen gas (95\% oxygen) breathing was investigated with respect to atherosclerosis development. Plaque hypoxia was found to be present in murine atherosclerotic plaques from plaque initiation on. Also, carbogen breathing actually reduced plaque hypoxia and decreased necrotic core expansion, likely by improving macrophage efferocytosis potential in the plaque. Thus, plaque hypoxia is reversible and affects atherosclerotic plaque development. Additionally, as even early plaques (fatty streaks) were hypoxic, oxygen consumption in the plaque rather than oxygen diffusion distance seemed to determine plaque oxygenation.

Next, the effect of reducing cellular oxygen consumption on plaque hypoxia was investigated. Dietary nitrate has been shown to improve mitochondrial respiration thus resulting in similar energy levels, with reduced oxygen consumption in skeletal muscle. Therefore, dietary nitrate supplementation was studied in atherosclerosis development and plaque hypoxia, presented in chapter 4 . Prolonged dietary nitrate supplementation did not affect atherosclerotic disease burden or plaque hypoxia, nor did it exert obvious systemic side effects. While, short term dietary nitrate (2 weeks) increased plasma nitrate 
and nitrite levels, this effect was no longer observed upon prolonged dietary nitrate supplementation (14 weeks). These data suggest a compensatory downregulation of plasma nitrate and nitrite levels upon prolonged dietary nitrate supplementation. Also, initial increases in nitrate and nitrite levels were not sufficient to affect atherosclerotic plaque burden or plaque hypoxia. No conclusions on a role of cellular oxygen consumption in plaque progression can be drawn from this model.

In chapters $\mathbf{5}$ and $\mathbf{6}$, cellular oxygen consumption was reduced by interfering with intracellular hypoxic signaling. Deficiency of prolyl-hydroxylases 1 (PHD1) has been shown to reduce cellular oxygen consumption, by switching cellular metabolism towards glycolysis. Indeed, PHD1 deficiency could reduce atherosclerotic plaque hypoxia (chapter 5). However, profound cholesterol-lowering seen in PHD1 deficient mice obscured potential effects of hypoxia on plaque progression. In turn, PHD3 did not alter atherosclerotic hypoxia (chapter $\mathbf{6}$ ) and neither of the models in chapter $\mathbf{5}$ and $\mathbf{6}$ thus allow for solid conclusions on the causal contribution of cellular oxygen consumption to plaque progression. Overall, these data suggest a more modulatory function of plaque hypoxia, which is easily overruled by other atherogenic players.

In chapter 5 the mechanism of plasma cholesterol-lowering by PHD1 deficiency was further investigated. Also the role of hematopoietic PHD1 deficiency as opposed to whole body PHD1 interference in atherosclerosis and cholesterol-lowering was studied. Cholesterol-lowering was mainly mediated by the stromal compartment lacking PHD1 and was shown to be mediated by enhanced cholesterol efflux in the feces by elevated trans-intestinal cholesterol efflux. The cholesterol-lowering seen seemed to be independent of downstream hypoxia-inducible factor stabilization.

In turn, whole body PHD3 deficiency slightly increased plasma cholesterol levels, as presented as initial findings in chapter 6. Additionally, hematopoietic PHD3 deficiency enhanced atherosclerotic disease burden and intra-plaque apoptosis, through a yet unknown mechanism. Whole body PHD3 deficiency in turn, did not alter atherogenesis. These two chapters demonstrated great diversity in PHD isoform effects on atherosclerotic plaque progression, hypoxia and lipid metabolism.

In chapter 7, the experimental findings of chapters 2 to 6 and their implications were discussed. The studies presented in this dissertation suggest a modulatory role of plaque hypoxia in atherosclerotic disease progression. Other atherogenic factors, such as plasma cholesterol levels, clearly outrun potential hypoxia effects. Aside from hypoxia, the data showed PHD interference isoform- and cell type- specific effects on atherosclerosis 
development, hypoxia and cholesterol metabolism. In contrast, PHD inhibition seemed to improve glucose tolerance, reduce adiposity and potentially even alleviated diabetes independent of the PHD isoform (chapter 7). In sight of general PHD inhibitors undergoing clinical trials, isoform-specific and cardiovascular effects need to be evaluated in those studies. Along those lines PHD1 isoform-specific inhibition might present a valuable and attractive strategy for atherosclerosis and cholesterol metabolism. 


\section{SAMENVATTING}

Atherosclerose ontwikkelt zich onder invloed van verhoogde cholesterolwaarden in het bloed (hypercholesterolemie) en lokale, chronische ontstekingsreacties. Deze ziekte is de oorzaak van een beroerte of hartinfarct. De huidige behandeling bestaat uit het operatief verwijderen van de atherosclerotische plaque, en behandeling van risico factoren zoals verhoogde cholesterolwaarden, bloeddruk en suikerziekte (diabetes). Er is aangetoond dat atherosclerotische hypoxisch zijn, maar er is nog geen oorzakelijk verband aangetoond met de ontwikkeling van de atherosclerotische plaque. In dit proefschrift is de hypothese onderzocht of plaque hypoxie causaal gerelateerd is aan atherogenese.

Eerst is de literatuur over hypoxie en inflammatie bestudeerd (hoofdstuk 2). Deze literatuurstudie toonde aan dat de aanwezigheid van plaque hypoxie werd bevestigd in verschillende species. Hypoxie werd in vitro vooral bestudeerd in relatie tot macrofaag functie, de gevolgen van inflammatie en lipide metabolisme.

Om een causale relatie tussen hypoxie en atherogenese vast te stellen in dit proefschrift, werd op twee manieren plaque hypoxie verminderd. Enerzijds werd de beschikbaarheid van zuurstof verhoogd en anderzijds werd het zuurstof verbruik door cellen in de plaque verlaagd.

In hoofdstuk 3 werd het tijdsverloop en de locatie van hypoxie in een muismodel voor atherosclerose bestudeerd. Plaque hypoxie werd gedetecteerd vanaf de eerste plaque ontwikkeling en voornamelijk aanwezig in plaque macrofagen. Aangezien zelfs vroege plaques hypoxisch waren, wordt de zuurstof status van de plaque waarschijnlijk bepaald door verhoogde zuurstofconsumptie en niet verlaagde zuurstoftoevoer. In dit hoofdstuk werd ook het effect van verhoogde systemische zuurstoftoevoer op atherogenese in de muis bestudeerd door muizen carbogeen gas met 95\% zuurstof te laten inhaleren. Dit verminderde plaque hypoxie en de ontwikkeling van de necrotische kern, waarschijnlijk door de fagocytose van apoptotische cellen door plaque macrofagen te verbeteren. Plaque hypoxie is dus reversibel en oorzakelijk gerelateerd aan atherogenese in muizen.

Vervolgens werd het effect van verlaagde zuurstofverbruik op plaque hypoxie en atherosclerose bestudeerd. Er is aangetoond dat orale nitraat supplementatie de mitochondriale ademhalingsketen efficiënter maakt, wat resulteert in gelijke energie productie met een verminderde zuurstofconsumptie in skeletspieren. Het effect van orale nitraat supplementatie op atherogenese en plaque hypoxie werd dus bestudeerd in hoofdstuk 4. Langdurige supplementatie gedurende 14 weken leidde niet tot veranderingen in atherogenese en plaque hypoxie, noch tot systemische bijwerkingen. 
Ondanks verhoogde plasma nitraat en nitrietwaarden na korte supplementatie gedurende de eerste 2 weken, waren deze waarden niet meer verhoogd na 14 weken. Deze data suggereren dat er een compensatie heeft plaatsevonden wat leidde tot normalisatie van de verhoogde plasmawaarden tijdens langdurige supplementatie. Daarbij, was de initiële verhoging van nitraat en nitrietwaarden niet voldoenden om atherogenese of plaque hypoxie te beïnvloeden. Er kan op basis van hoofdstuk 4 dus geen conclusie getrokken worden over de rol van cellulair zuurstof verbruik in atherogenese.

In hoofdstuk 5 en $\mathbf{6}$ werd het cellulaire zuurstofverbruik verlaagd door interventies in de intracellulaire hypoxie signalering. Deficiëntie van prolyl-hydroxylase 1 (PHD1) is bewezen te leiden tot verlaagd cellulair zuurstof verbruik door energieproductie via zuurstof-besparende glycolyse te stimuleren in plaats van oxidatieve fosforylering. PHD1 deficiëntie in muizen verminderde inderdaad atherosclerotische plaque hypoxie (hoofdstuk 5), maar een verlaging van plasma cholesterol waarden maakte het onmogelijk om conclusie te trekken over de role van alleen plaque hypoxie op atherogenese. PHD3 deficiëntie in muizen had geen effect op plaque hypoxie (hoofdstuk 6). Er kunnen dus geen betrouwbare conclusies getrokken worden over de oorzakelijke rol van cellulair zuurstofverbruik in atherogenese op basis van de modellen beschreven in hoofdstuk $\mathbf{5}$ en 6. Samenvattend, deze data suggereren dat plaque hypoxie een modulerende functie in atherogenese hebben, naast de causale rol van bekende primaire risicofactoren zoals hypercholesterolemie.

In hoofdstuk 5 werd het mechanisme van de verlaagde plasma cholesterolwaarden door PHD1 deficiëntie verder bestudeerd. Ook werd de bijdrage van PHD1 deficiëntie in hematopoietische cellen aan het effect op cholesterol en atherogenese vergeleken met PHD1 in niet-hematopoietische, stromale cellen. De verlaging van cholesterol werd vooral veroorzaakt door PHD1 deficiëntie in stromale cellen en werd gemedieerd door verhoogde trans-intestinale cholesterol efflux naar de feces. De waargenomen cholesterolverlaging lijkt onafhankelijk van de bekende PHD1 effecten op stabilisatie van hypoxie-induceerbare factoren.

In tegenstelling tot effecten van PHD1 op cholesterol, resulteerde deficiëntie van PHD3 in alle lichaamscellen van de muis in licht-verhoogde plasma cholesterolwaarden, maar niet tot effecten op atherogenese (hoofdstuk 6). Daarentegen, leidde PHD3 deficiëntie in hematopoietische cellen tot grotere atherosclerotische plaques met grotere necrotische kern en meer intra-plaque apoptose, door een nog onbekende moleculair mechanisme. Deze twee hoofdstukken laten de grote diversiteit in de effecten van de verschillende PHD isovormen op atherogenese, hypoxie en lipide metabolisme zien. 
In hoofdstuk 7, werden de experimentele resultaten uit hoofdstuk 2-6 en de implicaties bediscussieerd. De studies beschreven in dit proefschrift suggereren een modulerende rol van plaque hypoxie in atherosclerose, naast een primaire rol van bekende risicofactoren zoals plasma cholesterol. Naast conclusies over plaque hypoxie, beschrijft dit proefschrift PHD isovorm- en celtype-specifieke effecten op atherogenese, hypoxie en cholesterol metabolisme. In tegenstelling tot differentiële effecten van de PHD isovormen op atherogenese en cholesterol, leidde deficiëntie van elke PHD isovorm tot verbeterde glucose tolerantie en diabetes, en verminderde groei van adipocyten (hoofdstuk 7). Aangezien breedspectrum PHD remmers in klinische studies bestudeerd worden, wordt aangeraden isovorm-specifieke en cardiovasculaire effecten in deze studies te evalueren. Gezien de voornamelijk positieve cardiometabole effecten van PHD1 deficiëntie in tegenstelling tot de gemengde effecten van PHD2 en PHD3, lijkt PHD1 isovorm-specifieke inhibitie de meest aantrekkelijke strategie voor de behandeling van atherosclerose en hypercholesterolemie. 


\section{ZUSAMMENFASSUNG}

Aderverkalkung entwickelt sich unter Einfluss erhöhter Cholesterinwerte im Blut und lokaler, chronischer Entzündungsreaktion in der Arterie. Aderverkalkung ist die unterliegende Ursache von Schlaganfällen oder Herzinfarkten. Heutzutage behandelt man Aderverkalkungen vor allem durch operatives Entfernen der Ablagerungen (Plaque), und indem man versucht Risikofaktoren, sowie erhöhte Cholesterinwerte, Bluthochdruck und Diabetes zu minimieren. Als alternatieve haben wir in dieser Arbeit Sauerstoffmangel (Hypoxie) in den Ablagerungen/Plaques studiert. Es konnte schon gezeigt werden, dass die Plaques hypoxisch sind, aber ob diese Hypoxie an dem Krankheitsbild Aderverkalkung beiträgt, war noch nicht bekannt. Darum wurde in dieser Dissertation die Hypothese untersucht, dass Plaque Hypoxie ursächlich zu der Entwicklung und Verschlechterung der Aderverkalkung beiträgt.

Erst wurde eine Literaturstudie ausgeführt (Kapitel 2). In dieser Literaturstudie wurde deutlich, dass Plaque Hypoxie in Menschen aber auch in anderen Tiermodellen schon gezeigt werden konnte. Desweiteren, wurde bis jetzt vor allem der Effekt von Hypoxie auf „Makrofagen“ (Aufräumer-zellen in der Arterienwand) untersucht und festgestellt, dass diese Zellen in Hypoxie entzündende Eigenschaften annehmen und Fette aufnehmen.

Um einen direkten ursächlichen Verband zwischen Hypoxie und Aderverkalkung festzustellen, wurde in dieser Dissertation von zwei grundsätzlichen Methoden um Plaque Hypoxie zu verkleinern Gebrauch gemacht: Einerseits wurde die Zufuhr van Sauerstoff zur Aderverkalkungsablagerung erhöht und anderseits wurde der Gebrauch von Sauerstoff in der Plaque verkleinert.

In Kapitel 3, wurde der Verlauf der Plaque Hypoxie im Verband mit dem Plaque Wachstum untersucht. Zusätzlich wurde der Effekt von Sauerstoff atmen (Carbogen Gas, 95\% Sauerstoff) auf Aderverkalkung Entwicklung in Mäusen untersucht. Plaque Hypoxie konnte schon in sehr frühen Stadien der Aderverkalkungsentwicklung festgestellt werden. Desweiteren, konnte erhöhte Sauerstoff Zufuhr durch Carbogen Gas zu atmen, Aderverkalkungsentwicklung und vor allem Zelltod in der Arterie leicht vermindern. Dies war zum Teil einer erhöhten Aufraeum-funktion (Efferocytose) von Makrofagen in der Plaque zu zu schreiben. Ausserdem, da schon anfängliche Plaques hypoxisch waren, ist wahrscheinlich nicht die Sauerstoff Zufuhr aus der Blutbahn, sondern erhöhter Sauerstoff Gebrauch in den Plaques verantwortlich für die Plaque Hypoxie. 
Daraufhin wurde vermindern des Sauerstoffgebrauchs in den Plaques untersucht. Es konnte schon gezeigt werden, dass zusätzlichen Zufuegen von extra Nitrat in Trinkwasser die Effizienz der mitochondrialen Atmungskette erhöht und damit mit weniger Sauerstoffverbrauch die gleiche Energie in Zellen erzeugt wird. Darum wurde der Effekt von extra Nitrat in Trinkwasser auf Aderverkalkungsentwicklung in Mäusen untersucht (Kapitel 4). Langzeitiges zufuegen von nitrat hatte hier keinen Einfluss auf die Aderverkalkung. Nach 2 Wochen Supplementation, wurden erhöhte Nitratund Nitrietwerte im Blut festgestellt, während die Blutwerte nach 14 Wochen Nitrat Supplementation wieder gesackt waren. Diese Daten deuten auf eine Kompensierung der Blut Nitrat- und Nitrietwerte hin, was erklären könnte warum es keinen Unterschied in der Entwicklung von der Aderverkalkung gab. Von diesen Daten kann darum auch keine Aussage über den Einfluss von zellulärem Sauerstoff Gebrauch auf Plaque Hypoxie und Krankheitsbildentwicklungen gemacht werden.

In Kapitel 5 und 6, wurde zellulärer Sauerstoff Gebrauch vermindert in genetischveränderten Mäusen. Es war schon bekannt, dass das Ausschalten von dem Enzym Prolyl hydroxylase 1 (PHD1) den zellulären Energiehaushalt vom Gebrauch der Sauerstoff-konsumierenden mitochondrialen Atmungskette zur anäroben Glycolyse umschaltet. In der Tat, konnte PHD1 Defizienz in der Maus sowohl die Plaque Hypoxie als auch Aderverkalkungsentwicklung verkleinern (Kapitel 5). Allerdings hatten PHD1 defiziente Mäuse auch niedrigere Blut Cholesterinwerte, was wahrscheinlich die kleineren Plaques erklärt. Ein verwandtes Enzym, PHD3, konnte andererseits Plaque Hypoxie nicht beeinflussen (Kapitel 6). Auch von diesen Studien (Kapitel 5 und 6), kann also keine Aussage gemacht werden ob Plaque Hypoxie ursächlich zur Aderverkalkungsentwicklung beiträgt. Die Daten suggerieren aber, dass der Einfluss von Hypoxie nicht allzu gross ist und das andere Faktoren, wie erhöhte Cholersterinwerte, die Effekte von Hypoxie überschatten.

In Kapitel 5 wurde dann weiter untersucht, wie PHD1 Defizienz Cholesterinwerte beeinflussen kann. Desweiteren wurde der Einfluss von Immunzellen mit PHD1 Defizienz im Vergleich zur PHD1 Defizienz in allen Zellen untersucht. PHD1 Defizienz in nur den Immunzellen hatte hierbei wenig Einfluss auf Cholesterinwerte und Aderverkalkungsentwicklung. In der Tat war erhöhter Transport von Cholesterin direkt vom Blut über die Darmwand und nachfolgendes Ausscheiden im Stuhl die Ursache für niedrigere Cholesterinwerte in PHD1 defizienten Mäusen.

Interessanterweise, wurde das Gegenübergestellte gefunden in PHD3 defizienten Mäusen, die selbst erhöhte Cholesterinwerte zeigten (Kapitel 6). Hier sorgten vor 
allem die PHD3 defizienten Immunzellen für grössere Aderverkalkungsformung und erhöhten Zelltod in der Arterienwand. Der Mechanismus, wie PHD3 dieses verursacht, ist noch nicht bekannt. Diese zwei Kapitel zeigen aber, dass die PHD Enzym Familie „Isoform“ (PHD1, PHD3)-spezifische Funktionen in Aderverkalkungsentwicklung und Cholesterin Regulierung haben.

In Kapitel 7 wurden die experimentellen Ergebnisse der Kapitel 2-6 diskutiert und Schlussfolgerungen gezogen. Die Studien dieser Dissertation suggerieren eine gemässigte und regulierende Rolle der Plaque Hypoxie in der Aderverkalkungsentwicklung. Andere Faktoren, sowie erhöhte Cholesterinwerte, haben einen grösseren Einfluss auf das Krankheitsbild. Die Ergebnisse zeigen spezifische Effekte von PHD Enzym Isoformen auf Plaque Hypoxie, Aderverkalkungentwicklung und Cholesterinwerte. Gleichzeitig, scheinen alle PHD Isoformen eine ähnliche Funktion in Diabetes zu haben (untersucht in Kapitel 7). Hemmstoffe (Inhibitoren), die alle PHD Enzyme hemmen werden derzeit in Klinische Studien getest. Die Ergebnisse dieser Dissertation zeigen, dass Herz-und Kreislauf Effekte dieser Hemmstoffe berücksichtigt und untersucht werden sollten. Desweiteren könnten PHD Isoform-spezifische Hemmstoffe eine attracktive Strategie gegen Aderverkalkung und erhöhte Cholesterinwerte darstellen. 



$$
\text { " - - }
$$





\section{VALORIZATION}

Valorization is the process of creating value from knowledge. This includes (I) making knowledge available and suitable for economic and societal exploitation and (II) translating this knowledge into products, services, processes or even new businesses (1).

In general a drawback and at the same time strong point of basic academic research is that acquiring new knowledge should be independent of a commercial ulterior motive and based on general curiosity and a hypothesis derived from prior data. This presents a drawback, when trying to communicate the impact of results or research ideas with the general public or third parties with a commercial interest. As a strong point, however, unbiased academic research opens up the opportunity of unforeseen findings and translations. In this chapter, I would like to discuss how the knowledge gained from this thesis has been utilized until now and what potential ways of valorization remain to be explored. For all arguments presented, the reader should keep in mind the extensive 15 year time line from basic research findings presented in this dissertation to an actual applicable drug in humans.

Atherosclerosis, as part of cardiovascular diseases, presents an enormous economic burden in the western world nowadays. More than 70 million adults in the United States have one or more cardiovascular diseases, with hypertension, coronary heart disease and stroke being the most prominent ones. Cardiovascular disease direct and indirect costs were estimated to exceed $\$ 400$ billion in 2006 (2). When studying only acute coronary syndromes, these diseases accounted an annual cost of approximately $\$ 75$ billion (3). These costs are attributable to hospitalization and professional care, medication, home healthcare and indirect costs. Indirect costs are generally defined as reduction in work productivity of the patient itself and the associated economic burden (4). While all this evidence clearly justifies research on atherosclerosis, the challenge is to find novel drug targets to reduce this disease and economic burden.

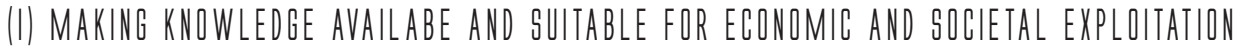

In this dissertation, we explored the role of hypoxia in atherosclerosis development and progression. All data presented here have been communicated on various (inter-) national conferences and chapters 2 to 5 have been published in peer-reviewed journals. Thereby, the knowledge is available to experts in the field and can be used as starting point and reference for future research. Also, it will minimalize chances of repetition of already performed research. This is particularly true for "negative data". In chapter 4, 
we showed a compensatory downregulation of plasma nitrate, nitrite and nitric oxide levels upon prolonged dietary nitrate supplementation. This makes future research using prolonged dietary nitrate in similar setups redundant. In line with that argument, we obtained a open access publication grant from ZonMW, more knowledge with less animals (MKMD), funding publication of neutral data to reduce use of animals. By publishing and communicating those data, we potentially prevented unnecessary animal experiments.

Besides academic and professional knowledge exchange, the data presented in chapters 2 to 5 were too preliminary to communicate with the general public. Nevertheless, I have gained experience in giving laymen talks on my research topics (TED-talk Prize, Vascular Biology, PhD-student course, Dutch Heart Foundation, The Netherlands) and have followed public writing courses (UM journal “Observant") to improve communication of research results in the future.

\section{(II) translating knowledge into products, services, processes and eVEN neW BUSINESSES}

Current therapies for atherosclerosis involve surgical removal of the plaque as well as risk factor management. In particular LDL-C lowering strategies, including statins and PCSK-9 inhibiting agents, have proven effective in reducing cardiovascular risk $(5,6)$. Anti-inflammatory agents are also undergoing clinical trials at the moment, however multiple agents have already failed to show a decrease in cardiovascular risk (7-10).

In this dissertation, we could show that reducing intra-plaque hypoxia by breathing of carbogen gas reduced atherosclerotic plaque burden and necrotic core expansion in a murine atherosclerosis model. Carbogen gas has already been successfully applied in hearing loss therapy (11) and hypoxia modification during radiotherapy (12) and has recently been tested in brain oxygenation for subsequent application to alleviate cerebral ischemia (13). Also in atherosclerosis, we could show a potential applicability of carbogen gas, as prolonged breathing reduced atherosclerotic plaque necrotic core expansion in mice. Though a translation of carbogen gas to human atherosclerosis remains to be shown, reversal of chronic intermittent hypoxia $(\mathrm{CIH})$ in apnea patients using continuous positive airway pressure (cPAP) could improve cardiac symptoms and hemodynamic parameters $(14,15)$. cPAP can increase daytime partial oxygen pressure by substantially reducing the number and severity of respiratory events in patients suffering from $\mathrm{CIH}$ (16). This knowledge suggests that methods to reduce intermittent hypoxia or atherosclerotic plaque hypoxia can indeed improve patient outcome. 
Considering the epidemic "atherosclerosis", oxygen therapy is however not feasible. In turn, downstream mechanisms affected by re-oxygenation might present a valid therapeutic target. In chapter 3 , we identified defective efferocytosis capacity via receptor MerTK downregulation in hypoxia. Restoring MerTK function might thus be able to reduce atherosclerosis progression. However, these results are highly preliminary and remain to be confirmed in animal models, before proceeding to the human situation. This knowledge might forward research into MerTK targeting therapies in atherosclerosis management.

As an alternative for oxygen therapy, we aimed to reduce cellular oxygen consumption via multiple approaches. None of the approaches used could identify a clear-cut role for hypoxia in atherosclerosis progression, suggesting a more modulatory function of plaque hypoxia. Interestingly, noninvasive imaging of hypoxia has recently been successfully used and correlated with inflammation in human atherosclerotic plaques (17). The potential of hypoxia imaging as diagnostic or prognostic marker remains to be shown.

More promising as an intervention for atherosclerosis and dyslipidemia, we established a new role of oxygen sensor PHD1 in cholesterol metabolism in this dissertation. PHD1 deficiency resulted in profound plasma cholesterol level lowering, predominantly in the VLDL and LDL fraction. Epidemiological evidence inevitably link high LDL-C levels with increased cardiovascular risk and vice versa, lowering LDL-C using statins has been shown to reduce cardiovascular risk. The identified new player, PHD1, thus likely acts independent of statins and also PCSK-9, as it targets a different route in cholesterol processing, namely trans-intestinal cholesterol efflux. This makes PHD1 inhibition an attractive target as adjuvant therapy for statin-resistant patients, which we are exploring at the moment (chapter 7). This project allowed us to obtain a grant-4-targets grant from Bayer (10.000€, Co-PI), already showing the interest of the pharmaceutical industry to collaborate.

Pan-PHD inhibitors, selective for PHD2, are currently undergoing clinical trials as erythropoiesis-stimulating agents in the treatment of anemia (www.clinicaltrials.org). This already shows the drugability and short-term safety of interfering with PHDs systemically. Throughout this dissertation, we could show PHD isoform- and cell typespecific effects on atherosclerosis development and cholesterol metabolism. In line, PHD2 and PHD3 but not PHD1 deficiency affected erythropoiesis. Thus, we aim to make a specific PHD1 inhibitor, not affecting erythropoiesis along with cholesterol lowering. We aim to use the resultant inhibitor for possible testing in clinical trials. On 
top of that, isoform-specific inhibitors might present a valuable research tool. In fact, making specific PHD inhibitors might present a feasible ground for starting up a spinoff company.

In conclusion, the findings of this dissertation show a minor role of hypoxia in atherosclerosis, which should be considered in future translational approaches. Alternatively, we identified a valid translational target in cholesterol metabolism, which we are further exploring at the moment. Future efforts include generating PHD isoformspecific inhibitors for clinical and basic research application.

A time line of the potential generation of an inhibitor is presented below (Figure 1). Upon discovery of a target, a drug needs to be developed, with subsequent in vitro and in vivo preclinical validation of specificity and drug activity. Subsequently, the drug must be optimized and screened for toxicological effects and formulated and manufactured for subsequent testing in clinical trials.

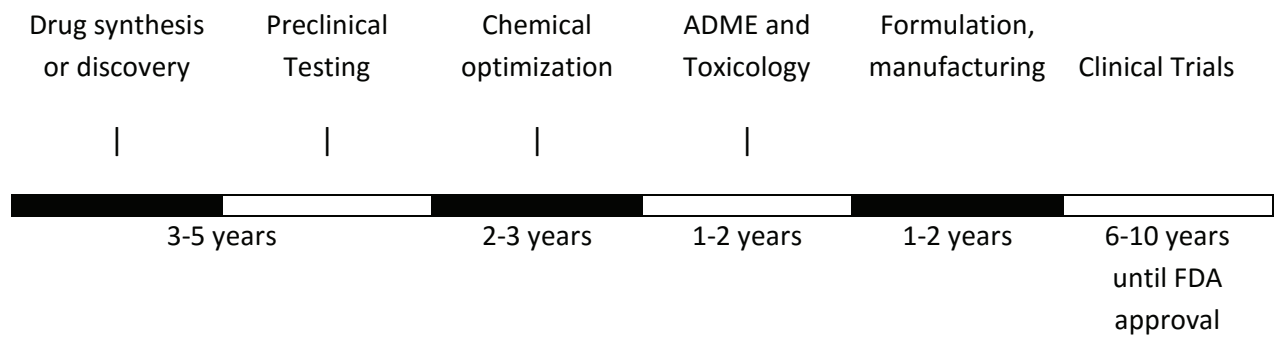

Figure 1: Drug discovery and development timeline, adjusted from (18).

* ADME = abbreviation for pharmacokinetics and pharmacology for "absorption, distribution, metabolism, and excretion. 


\section{REFERENCES}

1. Maastricht Valorisation Centre. Maastricht Valorisation Center - Maastricht University [Internet]. [cited 2016 Mar 10]. Available from: http://www.maastrichtuniversity.nl/web/show/ id $=7406582 /$ langid $=42$

2. Mensah GA, Brown DW. An overview of cardiovascular disease burden in the United States. Health Aff (Millwood). 2007 Jan 1;26(1):38-48.

3. Turpie AGG. Burden of disease: medical and economic impact of acute coronary syndromes. Am J Manag Care. 2006 Dec;12(16 Suppl):S430-4.

4. Boccuzzi SJ. Indirect Health Care Costs. Humana Press; 2003;63-79.

5. Everett BM, Smith RJ, Hiatt WR. Reducing LDL with PCSK9 Inhibitors--The Clinical Benefit of Lipid Drugs. N Engl J Med. 2015 Oct 22;373(17):1588-91.

6. ClinicalTrials.gov. Monoclonal Antibody Against PCSK9 to Reduce Elevated Low-density LipoproteinCholesterol (LDL-C) in Adults Currently NotReceiving Drug Therapy forEasing LipidLevels - Study Results - ClinicalTrials.gov [Internet]. [cited 2016 Feb 5]. Available from: https://clinicaltrials. gov/ct2/show/results/NCT01375777?sect $=X 70156 \&$ term $=$ PCSK9\&rank=16\# outcome1

7. Bäck M, Hansson GK. Anti-inflammatory therapies for atherosclerosis. Nat Rev Cardiol. Nature Publishing Group, a division of Macmillan Publishers Limited. All Rights Reserved.; 2015 Apr;12(4):199-211.

8. Bäck M, Yin L, Ingelsson E. Cyclooxygenase-2 inhibitors and cardiovascular risk in a nationwide cohort study after the withdrawal of rofecoxib. Eur Heart J. 2012 Aug;33(15):1928-33.

9. Nicholls SJ, Kastelein JJP, Schwartz GG, Bash D, Rosenson RS, Cavender MA, et al. Varespladib and cardiovascular events in patients with an acute coronary syndrome: the VISTA-16 randomized clinical trial. JAMA. 2014 Jan 15;311(3):252-62.

10. Tzellos T, Kyrgidis A, Zouboulis CC. Re-evaluation of the risk for major adverse cardiovascular events in patients treated with anti-IL-12/23 biological agents for chronic plaque psoriasis: a metaanalysis of randomized controlled trials. J Eur Acad Dermatol Venereol. 2013 May;27(5):622-7.

11. Yip K, Alonzi R. Carbogen gas and radiotherapy outcomes in prostate cancer. Ther Adv Urol. 2013 Feb;5(1):25-34.

12. Lee H-J, Park CY, Lee JH, Yang H-S, Kim JH, Ban MJ, et al. Therapeutic effects of carbogen inhalation and lipo-prostaglandin E1 in sudden hearing loss. Yonsei Med J. 2012 Sep;53(5):999-1004.

13. Ashkanian M, Borghammer P, Gjedde A, Ostergaard L, Vafaee M. Improvement of brain tissue oxygenation by inhalation of carbogen. Neuroscience. 2008 Oct 28;156(4):932-8.

14. Buchner NJ, Quack I, Woznowski M, Stahle C, Wenzel U, Rump LC. Microvascular endothelial dysfunction in obstructive sleep apnea is caused by oxidative stress and improved by continuous positive airway pressure therapy. Respiration. 2011/02/12 ed. 2011;82(5):409-17.

15. Drager LF, Bortolotto LA, Figueiredo AC, Krieger EM, Lorenzi GF. Effects of continuous positive airway pressure on early signs of atherosclerosis in obstructive sleep apnea. Am J Respir Crit Care Med. 2007 Oct 1;176(7):706-12.

16. Leech JA, Onal E, Lopata M. Nasal CPAP continues to improve sleep-disordered breathing and daytime oxygenation over long-term follow-up of occlusive sleep apnea syndrome. Chest. 1992 Dec;102(6):1651-5.

17. van der Valk FM, Sluimer JC, Vöö SA, Verberne HJ, Nederveen AJ, Windhorst AD, et al. In Vivo Imaging of Hypoxia in Atherosclerotic Plaques in Humans. JACC Cardiovasc Imaging. 2015 Nov 5;8(11):1340-1.

18. Sukhai MA, Spagnuolo PA, Weir S, Kasper J, Patton L, Schimmer AD. New sources of drugs for hematologic malignancies. Blood. American Society of Hematology; 2011 Jun 23;117(25):6747-55. 



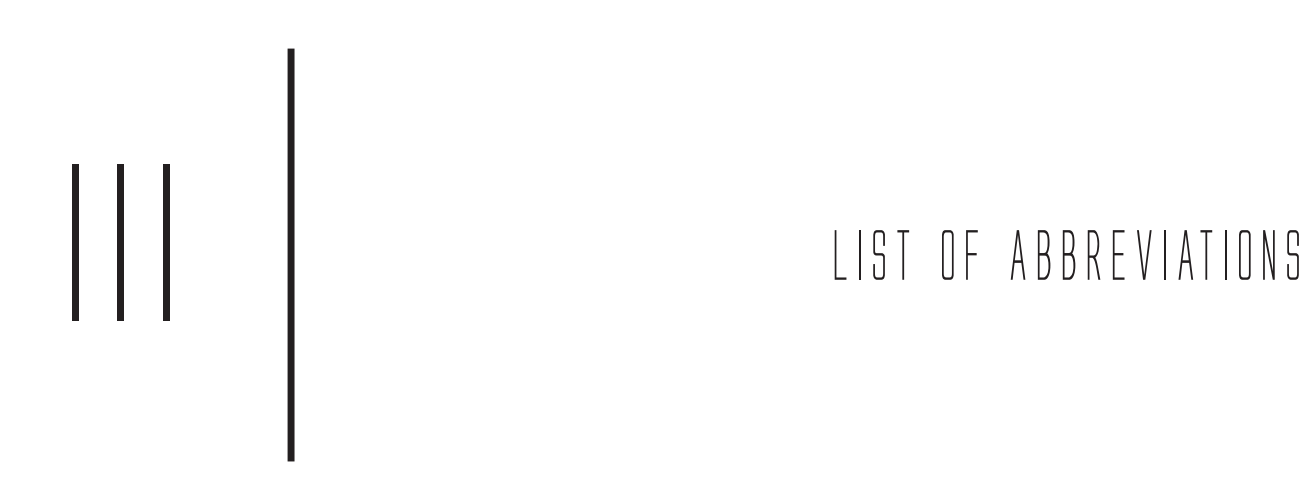



LIST OF ABBREVIATIONS

$\left[{ }^{14} \mathrm{C}\right] \mathrm{CO}$

$\left.{ }^{3} \mathrm{H}\right] \mathrm{TO}$

2-OG

ABCA1

ABCG 5/8

ACAT2

ApoE

apoER2

ASO

BMDM

CAD

CD36

CETP

$\mathrm{CIH}$

COX-2

cPAP

CVD

DMOG

EGLN

eNOS

EPR

$\mathrm{Fe}^{2+}$

$\mathrm{FH}$

$\mathrm{FIH}$

FOXO3

HBOT

HCD

HDL

$\mathrm{HIF}$

HMG-CoA

$\mathrm{I} / \mathrm{R}$

$\mathrm{IKK} \beta$

Irs2

$\mathrm{KO}$

$\mathrm{KI}$

LDL-C

LDLr $\left[{ }^{14} \mathrm{C}\right]$ cholesteryl oleate

Glycerol tri $\left[{ }^{3} \mathrm{H}\right]$ oleate

2-oxoglutarate

ATP-binding cassette, subfamily A, member 1

ATP-binding cassette subfamily $G$ member 5/8

Acyl-CoA cholesterol O-acyltransferase 2

Apolipoprotein E

Apolipoprotein E receptor 2

Antisense oligonucleotides

Bone marrow derived macrophages

Coronary Artery Disease

Cluster of Differentiation 36

Cholesterylester transfer protein

Chronic intermittent hypoxia

Cyclooxygenase 2

Continuous positive airway pressure

Cardiovascular Disease

Dimethyloxallyl Glycine

Egg-laying abnormal 9 homolog

Endothelial nitric oxide synthase

Electron paramagnetic resonance

Ferrous Iron

Familial hypercholesterolemia

Factory inhibiting HIF

Forkhead box O3

Hyperbaric oxygen treatment

High cholesterol diet

High density lipoprotein

Hypoxia-inducible factor

3-hydroxy-3-methylglutaryl-coenzyme A

Ischemia/reperfusion

Inhibitory kappaB kinase-beta

Insulin receptor substrate 2

Knockout

Knockin

Low Density Lipoprotein-Cholesterol

Low density lipoprotein receptor 
LPL

LRP

LXR

MAP

$\mathrm{MCH}$

$\mathrm{MCV}$

Mer'TK

MI

MMP

MTP

$\mathrm{NaCl}$

$\mathrm{NaNO}_{3}$

$\mathrm{NF} x \mathrm{~B}$

NITP

$\mathrm{NO}$

NPC1L1

ORO

OSA

oxLDL

OXPHOS

PAD

PCSK-9

PFKFB3

PHD

PPAR

RCT

RNI

ROS

SMCs

SPLA2

SRA

TAM

TF

TG

TICE

TLT

TSH

T $\beta$-MCA
Lipoprotein lipase

Low density lipoprotein receptor-related protein

Liver $\mathrm{x}$ receptor

Mean arterial pressure

Mean corpuscular hemoglobin

Mean cell volume

Mer tyrosine kinase

Myocardial infarction

Matrix metalloproteinase

Microsomal triglyceride transfer protein

Sodium-chloride

Sodium-nitrate

Nuclear factor kappa B

7-(4'-(2-nitroimidazol-1-yl)-butyl)-theophylline

Nitric oxide

Niemann-Pick C1-like 1

Oil red O

Obstructive sleep apnea

Oxidized LDL

Oxidative phosphorylation

Peripheral Artery Disease

Proprotein convertase subtilisin-kexin type 9

6-phosphofructo-2-kinase-fructose-2,6-biphosphatase 3

HIF-prolyl hydroxylase

Peroxisome proliferator-activated receptor $\delta$

Reverse Cholesterol Transport

Reactive nitrogen intermediates

Reactive oxygen species

Smooth Muscle Cells

Secretory phospholipase A2

Scavenger receptor A

Tumor-associated macrophage

Tissue factor

Triglyceride

Trans-intestinal cholesterol efflux

Thomas Lukas - the bull - Theelen

Thyroid stimulating hormone

Tauro-beta-muricholic acid 
UCP-1

VEGF-A

vHL

VLDL

WT
Uncoupling Protein 1

Vascular endothelial cell growth factor A

Von Hippel-Lindau protein

Very low density lipoprotein

Wild-type 



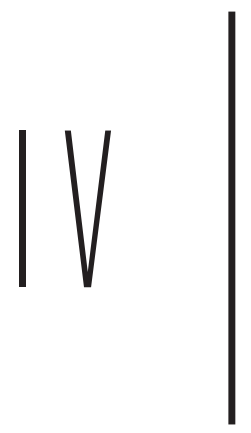

\section{ACKNOWLEOgMENTS \\ DAWKWOORD \\ dankgagung}





\section{ACKNOWLEDGMENTS I DANKWOORD I DANKSAGUNG}

None of the work in this dissertation would have been possible without the support and help of many people.

\section{Thank you!}

Co-promotor Dr. Judith Sluimer, beste Judith, het is echt gelukt!! Zelfs nog een last minute EHJ - ongelooflijk. De laatste maanden waren zeker hectisch en niet makkelijk voor je, en toch heb je alles gedaan en mogelijk gemaakt. Jij hebt mij ontzettend veel geleerd: wetenschappelijk, persoonlijk maar ook strategisch. En ik kan zeker nog veel meer van jou leren. Een betere begeleiding had ik me niet kunnen wensen! We hebben samen veel kunnen bereiken en ik je hebt me altijd gesteund en vertrouwd. Bedankt voor een hele leuke tijd! Wij blijven zeker in contact.

Promotor Prof. Mat Daemen, beste Mat, jouw laatste reddingsaktie was een last minute referentie schrijven. Bedankt, dat jij dit mogelijk hebt gemaakt. Ook gedurende mijn $\mathrm{PhD}$ heb je mij altijd goed begeleid vanuit Amsterdam. Met je helikopter view, wist je Thomas en mij bang te maken, maar ook onze projekten beter te maken en ons op het goede spoor te zetten. Van jouw strategisch en wetenschappelijk denken kan ik nog veel leren. Bedankt voor alle input en hulp.

Promotor Prof. Erik Biessen, beste Erik, meetings met jou waren altijd een oneindige bron van inspiratie en nieuwe ideeën, hoewel ik er waarschijnlijk maar maximaal 50\% van snapte. Bedankt voor alle input, die zeker in de laatste jaren aan de voortgang van mijn projekten heel erg heeft bijgedragen.

Dear members of the assessment committee, Prof. Post, Prof. De Windt, Prof. Tall, Prof. Kuivenhoven and Dr. Goossens, thank you very much for the very quick replies and comments from your sides. These have enabled me to apply for a FWO Marie-Curie fellowship, which means a great opportunity for me and my academic career. Thanks again.

Paranymph Thomas Lukas - the buizard - Theelen: Dank dir, habe ich die Promotion in Maastricht überhaupt erst angefangen. Vielen Dank! Deine Hilfe, Kollegialität, Freundschaft und Trinkfestigkeit haben uns gut durch die 4 Jahre gebracht und jetzt ist es doch komisch um nicht mehr zusammen zu arbeiten. Aber, wer weiss. ;) Ganz viel Erfolg mit dem Consulting! Wir bleiben in Kontakt, das ist ja wohl klar.

Paranymph Lauren, my Bestie! Where to start? There is just so much "back-story"... You always find a way to make us laugh and your collegiality and friendship have made my last two years in Maastricht an unbelievably happy time! Thank you very much for being such a great friend and for all your help! All the best for your PhD! 
Lieve collegas. Jeroen, Floor, David, Timo, Karen, Anette, Ine, Emiel, Anjana, Taghi, grosser Jan, Jasper, Kosta, Anke, Marchy, Bart en Margaux: het was altijd gezellig, ik kon altijd met vragen bij jullie terecht en ik ga het precieze aantal offeringen, die jullie met mij hebben gedaan, even niet noemen... En bovendien; jullie hebben altijd met produktief kommentaar vooruit geholpen en iedereen heeft zijn steentje bijgedragen om een hele leuke werk atmosfeer te creeëren. Dat is zeker niet overal standaard en heel erg bedankt hiervoor. Thank you very much.

Jasper, jij hebt de PHD1 en de PHD3 athero studies gesneden. Dat is veel werk, zoals je weet en heel erg bedankt daarvoor. Kosta, jij kon altijd voor grapjes zorgen, maar jij bent ook een hele goede onderzoeker! Succes met afronden van je $\mathrm{PhD}$ en met jullie paper - jullie hebben het zeker verdiend, met al het werk dat jullie hebben verzet. Marchy, (Sugar), I wish you a perfect start in Rostock: All the best with finishing your PhD and thank you for being a great colleage. Anke, jij hebt een moelijkere fase van je PhD achter de rug - veel succes met afronden! Bart, jij bent nu ook bijna klaar, veel succes met de laatste dingen! 'The "fresher" Margaux, you took over Thomas' seat and you know what responsibility comes with that;). I enjoyed the short time we worked together. I think you have a challenging project, but you will manage anyhow!

Verder wil ik de rest van de EVP bedanken: Sylvia, Marion, Marjo, Jack en Lieve, bedankt voor alle input en kritisch kommentaar. Ik heb extreem veel van jullie geleerd. Mat, Erwin, Clairy, Anique, Petra, Danielle, Elly en Cor. Jullie hebben mij allemaal altijd direkt geholpen en dat is heel fijn, want meestal wilde ik alles gisteren al klaar hebben. Bedankt voor het begrip en een goede samenwerking!

Mijn studenten zou ik ook graag willen bedanken: Hao en Bibian. You taught me a lot! You worked extremely hard, thank you very much. Ook een grote dank aan de indirekte studenten Levy, Simon, Erwin en Kees. Jullie hebben allemaal aan de data bijgedragen. Ook wil ik Prof. Axel zur Hausen, de epigenetica group, Axel's group en diagnostiek van de Pathologie bedanken voor een goede werkatmosfeer, hulpvaardigheid en interesse in mijn werk.

Aan alle medewerkers van het CPV: grote dank voor een hele goede samenwerking, special aan Richard, Saskia, Rik en Clarice, Paulien en Anouk. Jullie hebben altijd jullie best gedaan om alle experimenten mogelijk te maken en dat waren er best veel. Lieve I'mCARIM (ex-) leden Tom, Armand, Yvonne, Siamack, Emiel, Kosta, Margaux en Lauren en EPC leden. I very much enjoyed my time with I'mCARIM, thank you guys! I really think we achieved something with the fellowship and the industry workshop. Thank you very much for a great time. 
Furthermore, I would like to thank all co-authors who substantially contributed to this thesis and the publications. In het bijzonder, Prof. Albert Groen, Theo, Theo en Rick. Zonder jullie hadden wij het proces van PHD1 - gemedieerde cholesterol metabolisme niet gevonden. Bedankt voor de hulp en het harde werken. Ook uit Leiden kregen wij veel steun, van Prof. Patrick Rensen en Dr. Mariëtte Boon. Bedankt, jullie hebben bij het PHD1 verhaal sterk geholpen en voor een gezellige Gordon Conference en gezellige avonden in Maastricht gezorgd. Dr. Ludwig Dubois en Marike, jullie hebben mij altijd geholpen. Onder andere met medium, seahorse experimenten en pimonidazole aliquotjes. Bedankt hiervoor en succes met afronden van jouw $\mathrm{PhD}$, Marike! Dr. Kristiaan Wouters, jij hebt mij in het eind ook nog veel geholpen met de obesity studie. Dankjewel voor een goede samenwerking. Het zijn erg mooie data geworden. Dr. Steven Meex, bij de revisie van het EHJ stuk, maar ook eerder maakten wij vaak gebruik van jouw expertise en apparatuur bij de klinische chemie. Bedankt voor een goede samenwerking, ook dank aan jouw team. Prof. Carmeliet, u wil ik speciaal bedanken voor de kans een 3 maanden werkstage in uw groep te mogen lopen. Ik heb erg snel, veel bijgeleerd en hopelijk kunnen we dit vervolgen in een PostDoc.

Hier in Leuven habe ich auch tatkräftige Unterstützung erfahren: Ulrike und Lena, vielen Dank für die praktische Begleitung und eine tolle Zeit! Wir hoffen auf eine Verlängerung. Hartelijk dank ook aan Katie voor de organisatie!

Gerry, Rory en Hans - jullie ook bedankt voor de hulp met de Bayer grant en de eerste stappen in het maken van de PHD1 eiwit struktuur. Ben benieuwd of het ons lukt.

Prof. Schallreuter, Sie haben mich erst auf den Weg der Wissenschaft gebracht und stets begleitet. Vielen Dank für Ihre Unterstützung und „Mentorship“.

Lieve collegas van Biochemie, hoe vaak ben ik niet bij jullie iets komen halen? Dennis, Brecht, Tom, Armand, Rick, Leon en Niko. Dankjewel. Natuurlijk ook voor o.a. gezellige cocktail avonden ;). Sofie, Mike, Tom, Nadine van MolGen, jullie waren altijd ter plekke om te helpen, methanol te lenen, oil red o stainings te doen en protocollen uit te wisselen. Bedankt daarvoor! So, Freunde. Es ist geschafft.

All meinen Freunden ein herzliches Dankeschön für supertolle Promotionsjahre! John, Michael, Jennifer and Lauren - thank you for all the bullshit, chocomel parties, bike crashes, fog and Paulaner. Kevin und Caro, wie oft waren wir nicht bei euch eingeladen. Vielen Dank für eure gute Freundschaft! Oftmals auch zusammen mit Jo und Yara. Thanks! Thomas und Anna-Lena, ihr seid das witzigste Paar, das ich kenne. Vielen Dank für alle gute Laune, Leichtigkeit, gute Feiern und alles Gute in Brüssel für euch zwei! 
Desweiteren einen grossen Dank für den Freizeitsausgleich an (i) die Demminer (Tabby, Steffi, Claudi, mit Bifi und verpasstem Flieger in Berlin), (ii) die Pfortenser (Romy, Wiebke, Anja, Rike, viel Erfolg mit euren PhDs!!!), (iii) natürlich die Re-union Wochenenden vom Master Studium (Kata, Christopher, Hannah, Johanna, Anne, Maggi, Anna, Sophie und Jan), und (iv) spontane trips nach München oder wahlweise nach London zum shoppen mit Kristin. Vielen Dank euch allen!!

Lieve Floor, Rens en Oscar, jullie hebben op laatst moment nog mijn thesis design gemaakt. Het is prachtig geworden! Heel erg bedankt! Tammy, neben all den schönen Parties bei dir und dem Dinnerclub hast du mir mir unglaublich bei meiner These geholfen. Vielen Dank (zieht mal nach Leuven. ;).

Liebe Mama, lieber Papa. Auch, wenn ihr mir das nie glaubt, habt ihr unglaublich viel beigetragen zu dieser Doktorarbeit. Ihr habt den Grundstein dafür gelegt, dass ich mich frei entscheiden und entwickeln konnte und ihr habt mich immer getrieben, meine Ideen zu verwirklichen. Vielen Dank für all eure Unterstützung, Verständnis und euer unermüdliches Interesse in was ich denn eigentlich so den ganzen Tag mache. An meine lieben Grosseltern, Reimer und Lottemarie, Detlef und Anneliese: Ihr schafft es ausnahmslos die nicht allzu kleine Familie beisammen zu kriegen. Vielen Dank dafür und für euer Interesse in meine Arbeit. Meine grossen Brüder, Martin, Holger, liebe Juliane und Annija. Es ist soweit: Ich bin dann jetzt auch langsam fertig mit dem Studium ;). Ihr seid super!

Lieve Rens. Volgens mij weet jij nu alles over thesis schrijven, boek-kaft maken, beurzen aanvragen, muizen offeren... Ik weet niet hoe ik je moet danken voor alle steun, begrip en last minute hulp akties. Jij bent echt ongelooflijk.

THANK YOU VERY MUCH. 




$$
11=
$$



BIOGRAPHICAL SKETCH

\section{INSTITUTION AND LOCATION}

University of Maastricht (PhD)

The Netherlands

University of Maastricht (MSc)

The Netherlands

University of Durham (BSc)

United Kingdom

\section{REgEARCH EXPERIENCE}

MEAR(S)

Aug 2012 - May 2016

Sep 2010 - July 2012

Sep 2007 - June 2010
STUDY

Atherosclerosis

Biomedical Sciences

Specialization cancer

Biomedical Sciences

2012-16 PhD CARIM, Prof. Daemen, Prof. Biessen, Dr. Sluimer, University of Maastricht, The Netherlands

2016 Laboratory exchange, Prof. Carmeliet, VIB Leuven, Belgium

2015 Laboratory exchange, Prof. Groen, UMCG Groningen, The Netherlands

2012 Master internship, Dr. Sillessen, VUMC Amsterdam, The Netherlands

2011 Master internship, Dr. Wondergem, VUMC, Amsterdam,The Netherlands

2010 Laboratory Internship, Prof. Schmidt, University of Greifswald, Germany

2010 Bachelor Internship, Dr. Määttä, University of Durham, United Kingdom

2008 Laboratory Internship, Prof. Schallreuter, Institute for Pigmentary Disorders, Bradford, UK

2007 Nursing Internship, Evangelistic Hospital, Cologne, Germany

2004 Laboratory Internship, Prof. Schallreuter, Institute for Pigmentary Disorders, Bradford, UK

\section{AWARDS}

2015 Travel grant, Boehringer Ingelheim Fonds

2015 Grants 4 targets Bayer (co-PI, 10€k)

2015 Poster Price, ESC Summer School, Nice, France

2014 Audience Price, PhD-training courses, Papendal, Dutch Heart Foundation, The Netherlands

2013 Poster Price, TED-talk, PhD-training courses, Papendal, Dutch Heart Foundation, The Netherlands

\section{EXTRACURRICULAR ACTIVITIES}

Since 2014 PhD representative I'm CARIM, Maastricht, The Netherlands

2008 - 2009 Treasurer Biomedical Sciences Society, Durham, United Kingdom 


\section{PUBLICATIONS}

1. Marsch E, Demandt JAF, Theelen TL, Tullemans BME, Wouters K, Boon MR, van Dijk TH, Gijbels MJ, Dubois LJ, Hung G, Fisher EA, Biessen EAL, Daemen MJAP, Rensen PCN, Carmeliet P, Groen AK, Sluimer JC. Deficiency of the oxygen sensor prolyl hydroxylase 1 attenuates hypercholesterolemia, atherosclerosis and hyperglycaemia. accepted in Eur. Heart J.(2016) IF 15.2

2. Marsch E, Theelen TL, Janssen BJA, Briede JJ, Haenen GR, van Loon L, Poeze M, Bierau J, Gijbels MJ, Daemen MJAP, Sluimer JC. The effect of prolonged dietary nitrate supplementation on atherosclerosis development.

Atherosclerosis Dec;245:212-221. (2015) IF 3.9

3. Marsch E, Theelen TL, Demandt JA, Jeurissen M, van Gink M, Verjans R, Janssen A, Cleutjens JP, Meex SJ, Donners MM, Haenen GR, Schalkwijk CG, Dubois LJ, Lambin P, Mallat Z, Gijbels MJ, Heemskerk JW, Fisher EA, Biessen EA, Janssen BJ, Daemen MJ, Sluimer JC. Reversal of hypoxia in murine atherosclerosis prevents necrotic core expansion by enhancing efferocytosis.

Arterioscler Thromb Vasc Biol. Dec;34(12):2545-53. (2014) IF 6.0

4. Marsch E, Sluimer JC, Daemen MJ. Hypoxia in atherosclerosis and inflammation. Curr Opin Lipidol. Oct;24(5):393-400. (2013) IF 5.6

5. Schallreuter KU, Salem MAEL, Gibbons NCJ, Maitland DJ, Marsch E, Elwary SMA, Healey AR. Blunted epidermal L-tryptophan metabolism in vitiligo affects immune response and ROS scavenging by Fenton chemistry, part 2: epidermal H2O2/ONOO(-)mediated stress in vitiligo hampers indoleamine 2,3-dioxygenase and aryl hydrocarbon receptor-mediated immune response signaling.

FASEB. Jun;26(6):2471-85. (2012) IF 5.7 


\section{EXISTING MANUSCRIPTS}

1. Klinkenberg L, Luyten P, van der Linden N, Urgel K, Snijders D, Knackstedt C, Dennert R, Kietselaar B, Mingels A, Cardinaels E, Peeters F, van Suijlen J, ten Kate J, Marsch E, Theelen T, Sluimer J, Wouters K, Bekers O, Bekkers S, van Loon L, van Dieijen-Visser M, Meex S.

"Cardiac troponin T and I release following a $30 \mathrm{~km}$ run: comparison of troponin kinetics and effect of remote ischemic preconditioning"

Submitted.

2. Theelen TL, Marsch E, Demandt JA, Fallais S, Tullemans BM, van der Vorst EP, Donners MM, Gijbels MJ, Welting TJ, Reutelingsperger CP, Mastenbroek TG, Cosemans JM, Willems BA, Schurgers LJ, Carmeliet P, Biessen EA, Daemen MJ, Sluimer JC.

"Myeloid prolyl hydroxylase domain-containing protein 2 deficiency leads to larger, but more stable atherosclerotic plaques in mice“

In preparation.

3. Marsch E, Demandt JAF, Theelen TL, Tullemans BME, Heffron S, Janssen A, Rousch MJM, Fisher EA, Carmeliet PC, Heeneman S, Biessen EAL, Daemen MJAP, Sluimer JC.

"Hematopoietic HIF-prolyl hydroxylase 3 deficiency aggravates atherosclerotic plaque development"

In preparation. 


\section{LECTURES AND ABSTRACTS}

1. Marsch E, Demandt JAF, Theelen TL, Boon MR, Gijbels MJ, Dubois LJ, Carmeliet P, Fisher EA, Biessen EAL, Rensen PCN, Daemen MJAP, Groen AK, Sluimer JC.

"PHD1 deficiency promotes an atheroprotective metabolic phenotype"

Poster Prize, ESC Summer School, 14-18th June 2015, Nice, France.

2. Marsch E, Demandt JAF, Theelen TL, Boon MR, Gijbels MJ, Dubois LJ, Carmeliet P, Fisher EA, Biessen EAL, Rensen PCN, Daemen MJAP, Groen AK, Sluimer $\mathrm{JC}$.

"PHD1 deficiency promotes an atheroprotective metabolic phenotype"

Poster presentation, 17th International Symposium on Atherosclerosis, 23-25th May 2015, Amsterdam, The Netherlands.

3. Marsch E, Demandt JAF, Theelen TL, Gijbels MJ, Dubois LJ, Groen AK, Rensen PCN, Carmeliet P, Fisher EA; Biessen EAL, Daemen MJAP, Sluimer JC.

"PHD1 deficiency promotes an atheroprotective metabolic phenotype"

Poster presentation, Keystone - Hypoxia: From Basic Mechanisms to Therapeutics, 1216th May 2015, Dublin, Ireland.

4. Marsch E, Demandt JAF, Theelen TL, Gijbels MJ, Dubois LJ, Groen AK, Rensen PCN, Carmeliet P, Fisher EA; Biessen EAL, Daemen MJAP, Sluimer JC.

"PHD1 deficiency promotes an atheroprotective metabolic phenotype"

Audience Prize, PhD-training courses, Dutch Heart Foundation, 12-16th October 2014, Papendal, The Netherlands.

5. Marsch E, Demandt JAF, Theelen TL, Gijbels MJ, Dubois LJ, Rensen PCN, Carmeliet P, Fisher EA, Biessen EAL, Daemen MJAP, Sluimer JC.

"PHD1 deficiency promotes an atheroprotective metabolic phenotype"

Poster presentation, Gordon: Lipoprotein metabolism, 15-20th June 2014, Waterville Valley, NH, USA.

\section{Marsch E}

"Hypoxia and atherosclerosis"

Lecture, Hypertension Conference, Muenster, Germany. 


\section{Marsch E}

"Hyperbaric oxygen therapy for atherosclerosis"

Lecture, Symposium Hyperbaric Oxygen Therapy, 20th January 2014, AMC, Amsterdam, The Netherlands.

8. Marsch E*, Theelen T*, van Gink M, Jeurissen M, Verjans R, Janssen A, Otten JJ, Van der Loos CM, Wijnands E, Leenders PJ, Meex SJR, Donners MM, Dubois LJ, Lambin P, Mallat Z, Gijbels MJ, Fisher E, Biessen EA, Janssen BJ, Daemen MJ, Sluimer $\mathrm{JC} *$ contributed equally

"Reversal of murine plaque hypoxia prevents apoptosis and necrotic plaque expansion"

Poster Prize, PhD-training courses, Dutch Heart Foundation, 30th October - 4th September 2013, Papendal, The Netherlands.

9. Marsch E*, Theelen T*, van Gink M, Janssen A, Jeurissen M, Verjans R, Meex SJR, Dubois LJ, Lambin P, Gijbels MJ, Biessen EA, Janssen BJ, Daemen MJ, Sluimer JC, *contributed equally

"Reversal of murine plaque hypoxia prevents apoptosis and necrotic plaque expansion"

Oral presentation, Cardiovascular conference, 14-15th March 2013 Noordwijkerhout, The Netherlands. 